School of Biological Sciences

\title{
PHYLOGEOGRAPHY OF MAORICICADA CAMPBELLI (HEMIPTERA: CICADIDAE): MTDNA EVIDENCE INTERPRETED USING PHYLOGENETICS AND NESTED CLADE ANALYSIS
}

\author{
by \\ Kathy Barbara Rose Hill
}

A thesis

submitted to the Victoria University of Wellington

in fulfillment of the requirements for the degree of

Master of Science

in Ecology

March 2005 


\begin{abstract}
New Zealand, because of its wide range of habitats and rapidly changing, wellstudied palaeohistory provides an excellent opportunity to investigate the impact of earth processes on the distribution of taxa. There are now a variety of studies on plants that investigate such patterns, and a growing number of studies on animal taxa. Most studies have supported the hypothesis that current patterns of distribution of New Zealand taxa were created by dispersal, mediated by landscape and climatic conditions over time, and have contradicted the panbiogeographic hypothesis that explained distributions via vicariant landscape changes such as lateral displacement along the Alpine Fault. This thesis presents a detailed phylogeographic history of a widespread, endemic New Zealand cicada, Maoricicada campbelli, that is abundant throughout much of the South Island and is also found in the central volcanic plateau of the North Island. Mitochondrial DNA sequences of 223 individuals from 70 populations of $M$. campbelli were studied using both traditional phylogenetic methods and nested clade analysis (NCA). As found in a previous study of $35 M$. campbelli individuals, geographic structuring was strong, with two main clades (North Island+northern South Island versus Otago) representing diverse lineages that may in fact be different species. Population structuring within the northern South Island clade suggested that the central South Island was mostly uninhabitable during glacial periods and demonstrated a sister-group relationship between northern and southern Southern Alps populations to the exclusion of the more central Southern Alps populations. Population histories estimated from NCA support the hypothesis that most $M$.
\end{abstract}


campbelli populations were formed through dispersal rather than via fragmentation or Alpine Fault vicariance. Three areas of ring-species-like secondary contact were found between the Otago and northern South Island clades, between lineages that had been isolated for approximately 2.3my. Further study is predicted to confirm the presence of additional suspected contact zones, and will demonstrate whether the different lineages are reproductively isolated or hybridising at these areas of secondary contact. 


\section{ACKNOWLEDGEMENTS}

I am very grateful to the many people who helped with the collecting, labwork, analyses and writing of this thesis. I would especially like to thank my supervisor Chris Simon for many, many hours of discussion and advice on all aspects of this thesis, and for her time reading and re-reading the text. Chris also helped collect in the field, advised me on methods of analysis and topics of discussion, and provided me with the use of the laptop on which I finished the writing of this thesis. I would also like to thank my co-supervisor, Geoff Chambers, for discussions on the methods and analyses used in this thesis, for all the administrative work, and for the use of the IMS (Institute for Molecular Systematics) lab at VUW. My colleagues David Marshall and John Cooley of the University of Connecticut helped with the collecting and tape recording of specimens in the field, and also shared many discussions and discoveries on different aspects of cicada distribution and behaviour. Dave additionally provided assistance with some of the analyses. My examiners Phil GarnockJones and Pete Lockhart provided useful critiques on the earlier version of this thesis. The IMS lab supervisor Liz MacAvoy was invaluably helpful, providing assistance and information in all areas of labwork and with the editing of sequences, and I would like to thank her hugely for the many hours she spent with me. From initially knowing almost nothing of practical molecular biology, Liz trained me and helped me learn the many protocols needed for this thesis work. I would also like to thank the other students of IMS who provided help in the lab and the odd sequencing run. Several other people helped with the collecting of specimens, either with me in the field or on previous trips, especially Thomas Buckley, Peter Arensburger and Steve Chiswell. Roger 
Smith of GeographX, New Zealand, provided the Digital Elevation Maps or DEM map data of New Zealand used in Figs 3.1, 3.6 and 4.1. Harvey Collerton of the School of Earth Sciences (SES) helped me with the use of ArcView mapping software and SNAP software for converting GPS coordinates. Jill Ruthven of the SES library provided access to the detailed maps needed to find some old collecting localities. I would also like to thank the many other people at VUW, especially in the School of Biological Sciences (SBS) and Science Faculty who provided all kinds of assistance and information during my time there. Steve Chiswell and Elizabeth Cooke provided me with house-sitting opportunities, and Steve kindly allowed me to stay at his house for many months and even talk about cicadas occasionally. I would like to thank the New Zealand Marsden Fund and SBS for providing money for the labwork and travel to meetings, and the Marsden Fund and the US National Science Foundation (NSF) for providing travel money for fieldwork and some lab supplies. I would like to thank the New Zealand Department of Conservation (DoC), especially Ian Millar, who provided information and collecting permits for all sites within National Parks and other conservation lands. Finally, I would like to thank Chris Simon, my partner Dave Marshall and my parents, Peter and Barbara Hill, for providing me with the encouragement and threats needed to prevail. 
TABLE OF CONTENTS

Page

Abstract

Acknowledgements

Table of contents

Table, figure and appendix legends

i

iii

$\mathrm{v}$

vii

\section{Introduction}

1.1 Maoricicada campbelli

1.1.1 Prior Maoricicada campbelli analyses 2

1.1.2 New Maoricicada campbelli findings 2

1.2 New Zealand geography and palaeohistory 3

1.3 Hypotheses on the distribution of taxa throughout New Zealand 4

1.4 Nested clade analysis (NCA) 5

1.5 Previous phylogeographical studies of animal taxa in New Zealand 5

2. Methods 8

2.1 Collecting 8

2.2 Molecular labwork: extraction, amplifcation and sequencing of mtDNA 8

2.2.1 Genomic DNA extraction

2.2.2 PCR amplification and purification

2.2.3 Sequencing and alignment 11

2.3 Analyses

2.3.1 Analyses: phylogenetic methods

2.3.1.1 Maximum likelihood Analyses $\quad 12$

$\begin{array}{ll}\text { 2.3.1.2 Bayesian analyses } & 13\end{array}$

2.3.2 Analyses: phylogeographic methods (nested clade analysis) 14

2.3.2.1 Nested clade analysis network design 14

2.3.2.2 Rooting the nested clade analysis networks 15

2.3.2.3 Nested clade analysis using geographical associations 15

\section{Results}

3.1 Collecting

3.2 Molecular labwork: sequencing and alignment 23

3.3 Analysis results 24

3.3.1 Sequences: base composition and sequence divergences 24

3.3.2 Phylogenetic results (ML and Bayesian) 26

3.3.3 Phylogeographic nested clade analysis results 29

3.3.3.1 Nested clade analysis network diagrams 29

3.3.3.2 Nested clade diagram rooting 35

3.3.3.3 Nested clade analysis using geographical associations $\quad 37$ 
4.1 Summary of new Maoricicada campbelli findings 48

4.2 M. campbelli phylogeography and historical population movement 48

4.2.1 Overall findings $\quad 48$

4.2.2 Evolution of the North Island Clade 50

4.2.3 Evolution of the northern South Island clades 52

4.2.4 Evolution of the Otago clade $\quad 55$

4.3 Nested clade outgroup probabilities and rooting 56

4.4 New Zealand biogeography: dispersal versus vicariance 57

4.4.1 Hypotheses on the lack of endemism in the central South Island 59

4.4.1.1 Glacial hypothesis (dispersal) 59

4.4.1.2 Alpine Fault hypothesis (vicariance) 60

4.4.1.3 Testing the hypotheses (dispersal versus vicariance) 61

4.5 Contact zones

65

4.6 Ring Species 69

4.7 Potential speciation and song divergence in M. campbelli 72

5. Conclusions 75

5.1 Phylogenetic and phylogeography population structuring conclusions 75

5.2 Population movements: dispersal versus vicariance conclusions $\quad 75$

5.3 Contact zones and ring species conclusions 76

\section{References}

\section{Tables}

Table 3.1 Collecting localities and GPS data 18

Table 3.2 mtDNA variation within genes 25

Table 3.3 Total-mtDNA haplotype distances summary 25

Table 3.4 COI NCA geographical associations 40

Table 3.5 Total-mtDNA NCA geographical associations 41

\section{Figures}

Figure $3.1 \quad 22$

A. main clade locations and summary phylogeny,

B. population locations

Figure 3.2 ML and Bayesian phylograms 27

Figure $3.3 \mathrm{COI}$ nested cladogram 31

Figure 3.4 Total-mtDNA nested cladogram 33

Figure 3.5 Total-mtDNA Geodis output summary 38

Figure 3.6 Geographic location of South Island Total-mtDNA clades 46

Figure 4.1 Dispersal versus vicariance diagram of hypothesised clade 66 movements throughout New Zealand

\section{Appendices}

Appendix 2.1 Additional information on molecular labwork methods 90

Appendix 2.2 Mitochondrion map showing areas sequenced 92

Appendix 3.1 Additional information on molecular labwork results 93 


\section{Electronic Only Appendices (CD)}

Appendix 3.2 Total-mtDNA distance matrix

Appendix 3.3 Total-mtDNA haplotype alignment

Appendix 3.4 Haplotypes of all individuals sequenced

Appendix 3.5 Total-mtDNA and COI Geodis input and output

The included CD contains all the text, tables, figures and appendices presented in this thesis, plus an additional nested clade map. Electronic nested clade figures are especially useful because readers can zoom-in on small details.

\section{Table, figure and appendix legends presented in this thesis.}

Table 3.1. Maoricicada campbelli collecting localities (70 populations), GPS coordinates and haplotypes found in each population. Locations are sorted roughly north-south by district. District boundaries follow Crosby et al. (1998).

Table 3.2. Maoricicada campbelli mtDNA variation. All values are calculated with the seven overlapping bases at the end and start of $A 8$ and $A 6$ respectively included with A8. Gamma values estimated using the method of Frati et al. 1997. Maximum-step clades estimated using TCS 1.13.

Table 3.3. Summary of mtDNA distances shown in Appendix 3.3 from 71 TotalmtDNA Maoricicada campbelli haplotypes. Uncorrected distances are shown above distances corrected using ML (TrN+I model).

Table 3.4. Maoricicada campbelli nested clade geographical associations for COI sequence data estimated using the November 2001 Templeton key and Geodis output data. Clade names as in Fig 3.3. 
Table 3.5. Maoricicada campbelli nested clade geographical associations for Total-mtDNA sequence data estimated using the November 2001 Templeton NCA key and Geodis output data. Clade names as in Fig 3.4. See Fig 3.5 for a visual representation of clade geographical ranges.

Figure 3.1. A. Map of NZ showing the location of the main mtDNA clades and subclades of Maoricicada campbelli. A consensus tree showing the phylogenetic relationships is superimposed. B. Map of NZ showing all $70 \mathrm{M}$. campbelli populations. See Table 3.1 for more information and GPS coordinates. The dark line indicates both the main backbone of the Southern Alps and part of the Alpine Fault. The area between the dashed lines indicates the central South Island biotic gap. For both A. and B., red = northern South Island clade, green $=$ North Island clade, yellow $=$ Otago clade, orange indicates areas of secondary contact between the northern South Island and Otago clades. 3D DEM data provided by GeographX, NZ. NZ outline data provided by LINZ. Map produced using ArcView 3D analyst (ESRI).

Figure 3.2. Phylograms for 71 Maoricicada campbelli Total-mtDNA (COI+A6A8) haplotypes. A. Bayesian topology and branch lengths estimated from a partitioned-model with 4 million generations and a 10\% burn-in. Numbers next to nodes represent the Bayesian posterior probabilities. B. Maximum likelihood topology and branch lengths estimated from a $\mathrm{TrN}+\mathrm{l}$ model. Numbers next to nodes represent bootstrap values estimated from 44 pseudoreplicates. Grey highlighting indicates clades found with nested clade analysis, which are not necessarily monophyletic. Names in bold capitals represent the three main 
clades found in NCA analyses. $\mathrm{NI}=$ North Island, $\mathrm{SA}=$ Southern Alps, NMK = Nelson, Marlborough and Kaikoura.

Figure 3.3. Nested cladogram of 52 haplotypes of Maoricicada campbelli calculated by TCS from 753 bp of the COI mtDNA gene region. The 95\% parsimony connection limit $=11$ steps. Names represent unique haplotypes, circles represent inferred haplotypes. Straight lines between haplotypes indicate one mutational step. Dashed lines represent alternative connections that were disregarded using information from coalescent theory. Dashed boxes around haplotype names indicate unique tip haplotypes, each one step removed from the interior haplotype. Clade numbers are given as $\mathrm{Y}-\mathrm{X}$, with $\mathrm{Y}=$ number of the clade level, $\mathrm{X}=$ unique number within that level.

Figure 3.4. Nested cladogram of 71 haplotypes of Maoricicada campbelli calculated by TCS from 1520 bp of the COI and A6-A8 mtDNA gene regions. The $95 \%$ parsimony connection limit $=17$ steps. Names represent unique haplotypes, circles represent inferred haplotypes. Straight lines between haplotypes indicate one mutational step. Dashed lines represent alternative connections that were disregarded using information from coalescent theory. Dashed boxes around haplotype names indicate unique tip haplotypes, each one step removed from the interior haplotype. Clade numbers are given as $\mathrm{Y}$ $\mathrm{X}$, with $\mathrm{Y}=$ number of the clade level, $\mathrm{X}=$ unique number within that level. 
Figure 3.5. Summary of Geodis output results for NCA of 71 Total-mtDNA Maoricicada campbelli haplotypes. Clade names as in Fig 3.4. As noted elsewhere, the Otago and North Island clades are too divergent to be included in the same nesting sets as the northern South Island clades. See Appendix 3.5 for complete raw data. The letter I denotes interior clade, the letter T denotes tip clade. Numbers following clade names are geographical distances for "within clade", followed by "nested clade". Letters following geographical distances denote statistical significance, $\mathrm{L}=$ large, $\mathrm{S}=$ small.

Figure 3.6. Total-mtDNA nested clade geographic locations for South Island populations of Maoricicada campbelli. The location of all haplotypes and many higher clades are shown. Red with white internal are haplotypes, brown =1step clades, blue $=2$-step clades, green $=3$-step clades, orange $=4$-step clades. All localities shown on these two pictures, except for the Otago clades, are joined into the same 5 -step clade (5-1, northern-SI clade). Within text boxes white $=$ Southern Alps A and C, blue $=$ Southern Alps B, pink = Waitaki, yellow $=$ NMK and Otago. Some geographical features mentioned in the text are indicated. DEM courtesy of GeographX, NZ. 
Figure 4.1. Theoretical hypotheses of the distribution of Maoricicada campbelli over ca. 2.3my. Note that the present-day outline of New Zealand is used for all stages without reflecting known landscape changes over this time. Note also that clades are given their present-day range at all times. Ages of Otago and North Island clade separation events estimated by Buckley et al. (2001a). A dispersal scenario predicts that widespread $M$. campbelli were isolated in two refugia during the first glacial cycle, around 2.3mya. Note that the glacial refugium in the NMK area could have been in the North Island. The isolated Otago clade then remained static. The NMK clade dispersed to the North Island (or vice versa) approximately 0.9mya, then the NMK clade dispersed southward into the Waitaki and Southern Alps areas, coming into secondary contact with the Otago clade at the very end of this dispersal. A vicariance scenario predicts that a widespread $M$. campbelli species was subsequently fragmented into isolated populations evolving in situ. The Otago clade was the first to split off ca. 2.3mya, followed by the North Island clade ca. 0.9mya. The NMK clade split off, then lastly the Waitaki and Southern Alps clades separated. In the vicariance hypothesis, which requires the creation of barriers between populations, there would have been small scale dispersal around the edges to create secondary contact zones. Alternatively, population fragmentation could be postulated to follow a parapatric fragmentation model where a strong ecological gradient is coupled with assortative mating related to that gradient. In that case, contact zones would be primary. Inset: M. campbelli male. 
Appendix 2.1 Additional information on molecular labwork methods.

Appendix 2.2. Map of part of insect mitochondrion. Locations of primers and areas sequenced for Maoricicada campbelli shown.

Appendix 3.1 Additional information on molecular labwork results.

Appendix 3.2 Alignment of 71 Maoricicada campbelli haplotypes and two outgroups (Maoricicada clamitans (M.clamit) and Maoricicada phaeoptera (M phaeop) for 753bp of $\mathrm{COI}$ and 767bp of A6-A8. Blocks of haplotypes are shaded for ease of reading. The current gene is shown at the top of the page. Codons shaded black are those where the amino acid changes. The current number of bases is shown on the top right corner of each page. Note that codons in A8 are not aligned to spacing.

Appendix 3.3 Maoricicada campbelli mtDNA distance matrix for Total-mtDNA (COI+A6-A8) haplotypes.

Appendix 3.4 Sequenced individual Maoricicada campbelli COI, A6-A8 and Total-mtDNA haplotypes. Refer to Table 3.1 and Fig 3.1 for specific locality information.

Appendix 3.5 Geodis 2.0 input and output for COI and Total-mtDNA analyses of Maoricicada campbelli haplotypes. All output based on 10000 resamples. 


\section{INTRODUCTION}

\subsection{Maoricicada campbelli}

A population study of the endemic New Zealand cicada Maoricicada campbelli is a good example of a natural experiment in speciation theory. This species has been divided into different populations that have been evolving and dispersing over the landscape for at least 2.3my, over and around mountains, valleys, rivers and plateaus (Buckley et al. 2001a) and eventually establishing secondary contact as shown here. Throughout this time, changes in New Zealand habitats have been brought about by plate tectonics, vulcanism and climatic cycles. M. campbelli, like all other Maoricicada species, is present only on the largest two of the main islands, although cicadas of other genera have managed to spread to almost all other temperate New Zealand islands. $M$. campbelli is the most widespread member of its genus in both habitat tolerance and altitudinal, latitudinal and longitudinal range. There are few studies of cicadas to date that directly document either individual or population movement (but see Cumber 1952, Fleming and Scott 1970, Williams and Simon 1995 and references therein, Hill et al. submitted) but the strong geographic structuring found in this genus suggests a lack of dispersal. M. campbelli is one of the smallest Maoricicada species (body size 13-17mm), and (like most other Maoricicada spp.) possesses short wings relative to body size, which may reduce dispersal capabilities relative to other cicadas. Like other members of the genus, $M$. campbelli is mostly found on stony outcroppings and river outwash, but is much more flexible than other species, being found on dry farm pasture and rocky mountaintop scree, as well as river edges and glacial moraines in what was probably ancestral habitat (Fleming 1971). Host plants 
are currently unknown, but $M$. campbelli may oviposit and mature on a range of different plants that would allow occupation of their diverse habitats.

\subsubsection{Prior Maoricicada campbelli analyses}

Fleming (1971) suggested that $M$. campbelli evolved in an ice-age refugium in Otago and then expanded its range northwards, eventually dispersing to the central North Island volcanoes some 20,000 years ago. Buckley et al. (2001a) collected mitochondrial DNA (mtDNA) sequence information from 35 populations of M. campbelli (one individual per population) to test this idea. Their phylogenetic analyses separated these populations into five major clades and contradicted Fleming's hypothesis by suggesting that the current geographical range of $M$. campbelli is comprised of lineages that refuged in both northwest Nelson and Otago during Quaternary glacial maxima. Bayesian relaxed-clock dating estimated a divergence of $2.3 \pm 0.5 \mathrm{my}$ between the North Island+northern South Island and Otago populations (see Buckley et al. 2001a: Fig 1). They suggested that the most widespread clade dispersed south from the probable refugium in northwest Nelson, and currently surrounds the Otago clade on three sides. They hypothesised four contact zones but had direct evidence for none. Their molecular dating analysis suggested that the clade in the North Island was isolated for ca. 0.9my, managing to survive there throughout several glacial cycles.

\subsubsection{New Maoricicada campbelli findings}

Molecular phylogenetic evidence from 70 populations (223 individuals) of Maoricicada campbelli presented in this thesis supports the northwest Nelson 
and Otago refuge hypothesis of Buckley et al. (2001a) for the initial ice-ages, and suggests that there were several refugia in the more recent glacial maxima. These new data also establish the existence of three of the predicted contact zones (Buckley et al. 2001a), and use nested clade analysis to suggest more specific hypotheses of population movement. These analyses suggest that populations of the northern South Island clade spread southward, creating secondary contact zones between the farthest south populations and the outer edges of the Otago clade (Figs 3.1, 3.6). This indicates secondary contact of a ring-like form, where the most divergent populations meet after moving around a barrier. In this case a barrier of unsuitable habitat was presumably created by early ice ages that eliminated the M. campbelli populations in the centre of the South Island that initially linked the northern South Island and Otago clades together. The repeated elimination of populations in the central South Island area also provides support for a glacial theory for the distribution of $M$. campbelli in the South Island.

\subsection{New Zealand geography and palaeohistory}

New Zealand lies at the boundary of the Pacific and Australian plates and today comprises three main islands, many small inshore islands and several offshore islands. New Zealand broke from Gondwanaland around 80mya and reached its present location approximately 65mya (Cooper and Millener 1993). Although aspects of the geological and biological history of New Zealand throughout the late Mesozoic and Cenozoic are still debated (Fleming 1980, Thornton 1994), the history of the last $5 \mathrm{my}$ is better known (McGlone 1988, McGlone et al. 2001). This period includes all of the ca. 18 Quaternary glacial-interglacial 
cycles and most of the rapid mountain building of the Southern Alps. Although the Quaternary began 1.8mya, initial cooling began in the Pliocene approximately 2.5mya (Thornton 1994). The available terrestrial habitat of New Zealand changed with each glacial-interglacial cycle. The lowering of the sea level with glacial maxima increased the amount of coastal land and joined the three main islands. At the same time, the core habitable land was reduced by the formation of inhospitable glaciers along the Southern Alps and central North Island, surrounded by thousands of hectares of cold, dry tundra, plagued by gale force winds (Fleming 1980, Thornton 1994, Trewick and Wallace 2001). Since the start of the Kaikoura Orogeny around 25mya the Plates have slipped laterally, causing displacement along the Southern Alps of roughly $480 \mathrm{~km}$ and separating the Nelson and Otago areas that once lay adjacent (Sutherland 1994, 1999). The most intense period of mountain building in the Southern Alps occurred in the centre during the last 5-6my (Fleming 1980, Batt et al. 2000, Chamberlain and Poage 2000). McGlone et al. (2001) describe this central Southern Alps area as a single alpine zone in relation to its flora, and note that that there are no valleys descending below tree line for a ca. $200 \mathrm{~km}$ stretch.

\subsection{Hypotheses on the distribution of taxa throughout New Zealand}

Two models have been proposed for the distribution of biota throughout New Zealand: a vicariance approach that suggests that taxa were moved around passively through the formation and reduction of barriers, such as tectonic movement of the landscape; and a dispersal approach that suggests that taxa moved themselves, mediated by climatic, volcanic and tectonic events. In the 
central Southern Alps biotic gap zone, panbiogeographers suggest that lateral movement along the Alpine Fault resulted in this gap (e.g., Craw 1988,1989, Heads 1998), whereas other biologists propose a glacial theory, whereby taxa were eliminated from this area by severe glaciers and unstable glacial outwash during ice-ages. Detailed studies of population movement are needed to test these hypotheses.

\section{$\underline{1.4 \text { Nested clade analysis }}$}

Nested clade analysis (NCA) has been most effectively used to estimate the geographic history of populations within species, working best on widely distributed species with somewhat limited dispersal and only small amounts of genetic diversity (Templeton et al. 1987). Using sequences or restriction enzyme data, haplotypes are assembled into a cladistic network. These linked haplotypes are then grouped into clades, starting with haplotypes only one mutation apart, then moving to larger clades encompassing more and more haplotypes. These "nested clades" are then analysed using geographic distances within and between clades at different levels to estimate the evolutionary history of movement of the haplotypes to their present populations. The previous study (Buckley et al. 2001a) indicated that M. campbelli should be an ideal candidate for nested clade analysis.

\subsection{Previous phylogeographical studies of animal taxa in New Zealand}

Studies employing molecular methods to explore the phylogenetic and phylogeographic structure of New Zealand taxa are becoming more and more detailed and this study on $M$. campbelli represents one of the most detailed 
molecular studies of a single animal taxon within New Zealand. Trewick et al. (2000) studied the mtDNA of 21 populations of a species of alpine scree weta (Deinacrida connectens) that is found mostly at higher altitudes than $M$. campbelli. As found in M. campbelli (Buckley et al. 2001a and this study), populations of $D$. connectens from different areas showed much higher genetic divergence than that normally reported for intraspecific variation, however their estimated radiation time of about 4 mya ties population differentiation to mountain building rather than climate effects from glacial cycles. Trewick et al. (2000) found 36 different haplotypes, with the deepest genetic divergence found between populations that were also the most divergent geographically (Nelson and Southland). This scree weta is currently a high alpine taxon, and Trewick et al. (2000) suggest that the range of $D$. connectens is actually restricted during interglacials, and wider during glacial periods. Chin and Gemmel (2004) studied species of South Island cockroaches in the genus Celatoblatta. Their taxa show results somewhat consistent with the Maoricicada as a whole (Buckley et al. 2001b), with divergences between taxa around 4.2mya explained by central Southern Alps mountain building. One species ( $C$. hesperia), however, is found on both sides of the Southern Alps, with dating suggesting populations diverged during glacial cycles. The two Celatoblatta species found within the area of the M. campbelli Otago clade area were in one divergent group, however there was little sampling of this area. Chin and Gemmel (2004) also describe potential secondary contact and hybridisation within the central South Island biotic gap area (see Fig 3.1B), between species found north, south and centrally, and they suggest that the number of different haplotypes in this central area shows Celatoblatta survived there during glacial 
maxima. The phylogeography of the forest-restricted New Zealand short-tailed bat, Mystacina tuberculata, was studied using mtDNA control region sequences by Lloyd (2003). Lloyd analysed 241 individuals from 13 populations using various methods including nested clade analysis. Unfortunately for comparison with M. campbelli, most short-tailed bat populations are in the North Island, however the two South Island populations were unique. Hypotheses on the phylogeography of the South Island populations of the short-tailed bat suggested that they were probably isolated in a glacial refuge in the north of the South Island and then spread down either side of the Southern Alps during interglacials. Lloyd (2003) suggested that areas of the central North Island and most of the South Island experienced repeated introductions and extinctions throughout the glacial cycling. Interestingly, although Nothofagus existed south of the biotic gap area during at least the last glacial maximum, Lloyd (2003) argues that the climate in this area would have been too severe for populations of short-tailed bat to have survived.

Each of these papers demonstrates geographical structuring within animal taxa due to climatic or geological events, and in some cases with contact or hybridisation zones suggested, and potential cryptic species discovered. This study of M. campbelli also sought to understand population structuring within a widespread taxon; whether populations moved through vicariance or dispersal techniques; if there currently were or had been contact zones between populations and whether any populations had diverged enough to actually be cryptic species. 


\section{MetHODS}

\section{$\underline{2.1 \text { Collecting }}$}

Maoricicada campbelli males and females were collected from a range of localities throughout New Zealand. Collection sites were identified from historical records, a knowledge of habitat or from hearing the songs of the males. Individuals were captured by hand or with a net; males were located by sound, females were noticed incidentally in the course of collecting the males. Specimens were stored in individually labeled containers in $95 \% \mathrm{EtOH}$ at $-4^{\circ} \mathrm{C}$ until extraction was completed. They were then stored long-term at $-80^{\circ} \mathrm{C}$. The precise location of populations sampled was determined by using GPS in the field, or (for older samples) by extrapolating from high-definition maps if the GPS was unavailable. The GPS data were entered into ArcView (GIS software, ESRI) to error check the location of sites and to output site maps, and was used in the NCAs to calculate geographical distances.

Voucher specimens will be deposited in the New Zealand Arthropod Collection (NZAC), the Museum of New Zealand Te Papa Tongarewa collection and at the University of Connecticut.

\subsection{Labwork: extraction, amplification and sequencing of mtDNA}

\subsubsection{Genomic DNA extraction}

DNA was extracted from approximately $0.1 \mathrm{~g}$ of thorax muscle tissue using a Qiagen DNeasy ${ }^{\mathrm{TM}}$ Tissue Kit following the manufacturers instructions. The final elution was made into two $200 \mu \mathrm{L}$ aliquots of $\mathrm{dd}_{2} \mathrm{O}$, which were stored 
separately at $4^{\circ} \mathrm{C}$. When the work was completed, extractions were stored at $80^{\circ} \mathrm{C}$ for future use. Additional information on extraction methods trialled can be found in Appendix 2.1.

\subsubsection{PCR amplification and purification}

A polymerase chain reaction (PCR) method was used to amplify two portions of the mtDNA genome: approximately $800 \mathrm{bp}$ of the 3 ' end of the Cytochrome Oxidase subunit I (COI) gene was amplified using the conservative primers $\mathrm{C} 1$ J-2195 and TL2-N-3014 (Simon et al. 1994); and approximately 800 bp of a gene portion spanning the A6-A8 region (part of tRNA ${ }^{\text {Lys }}(\mathrm{tP})$, $\mathrm{tRNA}^{\mathrm{Asp}}$ (tK), ATPase subunit 8 (A8) and most of ATPase subunit 6 (A6)) was amplified using the primers TK-J-3799 and A6-N-4570 (Buckley et al. 2001a). Additional primers were constructed using Oligo 5 (primer analysis software, Molecular Biology Insights (MBI)) and named according to the convention set out in Simon et al. 1994. A second primer was constructed for the 3' end of the A6-A8 region from COIII sequence obtained by Thomas Buckley (unpublished data) and was used to amplify the A6-A8 region with problematic sequences. This primer (sequence 5'-ATACCAGATACAAAACTTAGAGC-3') was located near the 5' end of the COIII region and was named C3-N-4834. Internal primers were constructed to sequence the ends of the $\mathrm{COI}$ fragment in cases where base calls were disputed near the beginning or end of the sequence fragment, or when nuclear copies of mitochondrial genes (numts) were suspected. These internal primers were constructed from $M$. campbelli $\mathrm{COI}$ sequence data and named C1-J-2615 (sequence 5'-GCTATTATGGCAAGATTTATTCA-3') and C1N-2576 (sequence 5'-TAATGAAAATGAGCAACTAC-3'). 
The COI gene regions (whole and internal) were amplified using Qiagen Taq DNA Polymerase enzyme. The $25 \mu$ l reaction consisted of $2 \mu$ l of genomic DNA added to a $23 \mu \mathrm{l}$ reaction mix $\left(10.35 \mu \mathrm{ld}_{2} 0 ; 5.0 \mu \mathrm{l}\right.$ Qiagen PCR solution; $2.5 \mu \mathrm{l}$ Qiagen *10 PCR solution; $2 \mu$ l of each primer; $1 \mu$ l dNTP; $0.15 \mu$ l Qiagen Taq enzyme), covered with $20 \mu$ l mineral oil and cycled in a Perkin Elmer Thermal Cycler. Cycle sequence: initial: $94^{\circ} \mathrm{C}$ for 2 mins; then 30 cycles of: $94^{\circ} \mathrm{C}$ for 45 secs; $56^{\circ} \mathrm{C}$ for $45 \operatorname{secs} ; 72^{\circ} \mathrm{C}$ for $1 \mathrm{~min} 15$ secs; final extension: $72^{\circ} \mathrm{C}$ for 10 mins.

The A6-A8 gene region was amplified using either of two methods that worked equally well. 1) $1.5 \mu \mathrm{l}$ genomic DNA from extraction elution one was added to a 23.5 $\mu$ I PCR reaction mix using Amplitaq Gold enzyme and solutions $(14.42 \mu \mathrm{l}$ $\mathrm{ddH}_{2} 0 ; 2.4 \mu$ Amplitaq Gold PCR solution; $2.4 \mu \mathrm{l}$ Amplitaq gold MgCl solution; $1.0 \mu \mathrm{l}$ each primer; $2.0 \mu \mathrm{l}$ dNTP; $0.28 \mu \mathrm{l}$ Amplitaq Gold Taq enzyme) and run on a MJ Research Gradient PCR machine. Cycle sequence: initial: $94^{\circ} \mathrm{C}$ for 2 mins; then 30 cycles of: $94^{\circ} \mathrm{C}$ for 45 secs; $63^{\circ} \mathrm{C}$ for 45 secs; $72^{\circ} \mathrm{C}$ for $1 \mathrm{~min}$ 15secs; final extension: $72^{\circ} \mathrm{C}$ for 10 mins. 2) $2 \mu$ genomic DNA from extraction elution one was added to a $23 \mu \mathrm{l}$ PCR reaction mix using Amplitaq Gold master mix (12.5 $\mu$ I Amplitaq Gold master mix; $7.5 \mu l \mathrm{ddH}_{2} 0 ; 1.5 \mu$ l each primer) and run on a 2700 Thermal Cycler using the two-step method: initial : $94^{\circ} \mathrm{C}$ for 10 mins; then 30 cycles of $94^{\circ} \mathrm{C}$ for 15 secs; $64^{\circ} \mathrm{C}$ for $1 \mathrm{~min}$; final extension: $72^{\circ} \mathrm{C}$ for 7 mins. A $12.5 \mu \mathrm{l}$ PCR reaction mix reduced proportionally and used for the last A6-A8 amplifications worked equally well or better. Additional information on PCR amplification can be found in Appendix 2.1. 
PCR products were purified using the Roche Applied Science High Pure PCR Product Purification Kit following the manufacturers instructions and eluted into 8-20 $\mu$ l elution buffer.

\subsubsection{Sequencing and alignment}

Each specimen was sequenced in both directions for the COI gene region, and selected individuals were sequenced in both directions for the A6-A8 gene regions using a standard PCR cycle sequencing mixture (Bigdye version 2) and reaction, and then sequenced on a 377 Perkin Elmer automated sequencer with ABI Prism Sequence Analysis 3.4.1 software. Individuals with unreadable sequences, either from amplification/sequencing errors or sequences containing suspected numts were resequenced or sequenced with the internal (COI) or external (A6-A8) primers. Sequences that still proved unreadable were discarded. Additional information on obtaining readable sequence can be found in Appendix 2.1.

Sequences obtained by Buckley et al. (2001a) were used as templates for sequence alignment. DNAStar software was used to align the sequences, which were edited, translated, and checked manually. The forward and reverse for each individual $M$. campbelli $\mathrm{COI}$ and A6-A8 mtDNA region were aligned and checked, and then all sequences for each mtDNA region were aligned and assigned to specific haplotypes. 


\subsection{Analyses}

\subsubsection{Analyses: phylogenetic methods}

Phylogenetic analyses were performed using maximum likelihood (ML, Felsenstein 1981) implemented in PAUP* 4.01 (Swofford 1998) and Bayesian MCMC analyses using MrBayes 3.04b (Huelsenbeck and Ronquist 2001). All phylogenetic trees were rooted using the congeneric species $M$. clamitans and M. phaeoptera as in Buckley et al. (2001a). Base composition stationarity tests were run in PAUP* 4.01.

\subsubsection{Maximum likelihood analyses}

$M L$ analyses were performed on the Total mtDNA $\left(\mathrm{COI}+\mathrm{tRNA} \mathrm{Asp}_{+}+\mathrm{A} 6+\mathrm{A} 8\right)$ haplotype dataset. Modeltest (Posada and Crandall 1998) was used to choose the model of evolution $(\operatorname{TrN}+\mathrm{l})$ for the $\mathrm{ML}$ analysis. $\operatorname{TrN}$ is a modification of the GTR model, where only transitions (G-A, T-C) are estimated from the data and the other four rates are given as one. A heuristic search was completed using empirical base frequencies and other parameters estimated from Modeltest (G$A=27.543200, T-C=20.065200, I=0.7833)$, with the initial trees obtained via random stepwise addition followed by TBR branch swapping for 10 replicates. Statistical support for nodes was estimated using the nonparametric bootstrap (Felsenstein 1985), which was run using these same parameters for 44 pseudoreplicates. The large data set with its very closely related taxa meant that analyses were slow, hence the number of replicates was low. 


\subsubsection{Bayesian analyses}

The method of Frati et al. (1997) was used to choose the models for each partition used in Bayesian analyses. The model-testing method of Frati et al. (1997), like Modeltest, uses an algorithm to test whether one model is better than another, but unlike Modeltest starts with the most complex model and then tests it against nested models until it finds a significant difference in likelihood. The Akaiki Information Criterion (AIC) (Akaiki 1973, 1974) was also used in conjunction with this method to find the best model. The selected model is not significantly different in likelihood from the most parameter-rich model but itself has as few parameters as possible. Bayesian analyses were performed on a partitioned Total-mtDNA dataset and run for 4 million generations, sampling every 100 generations. There is no consensus on how to design data partitions for the best result. Adding parameters usually results in a higher likelihood, however using too many partitions can result in overparameterisation which causes a loss of power in the analysis (Lemmon and Moriarty 2004). A conservative model using three data partitions was chosen: coding DNA was split into $1^{\text {st }}+2^{\text {nd }}$ base pairs modelled with $\mathrm{HKY} 85+\mathrm{I}$ and $3^{\text {rd }}$ base pairs modelled with GTR $+\Gamma$, non-coding DNA (tRNA ${ }^{\text {Asp }}$ ) was modelled with HKY85+l. The program Tracer 1.1 (Andrew Rambaut and Alexei Drummond, University of Oxford, UK) was used to test the Bayesian datasets for stationarity and parameter autocorrelation. Branch support was estimated using Bayesian posterior probabilities. 


\subsubsection{Analyses: phylogeographic methods (nested clade analysis)}

\subsubsection{1 nested clade analysis network design}

Networks for COI, tRNA ${ }^{\mathrm{Asp}}, \mathrm{A} 6, \mathrm{~A} 8$, and Total mtDNA $\left(\mathrm{COI}+\mathrm{tRNA}^{\mathrm{Asp}}+\mathrm{A} 6+\mathrm{A} 8\right)$ haplotypes were constructed using the computer program TCS 1.13 (software for estimating gene genealogies, Clement et al. 2000). An unrooted network linking all haplotypes through a coalescent method that estimates the $95 \%$ probability of haplotypes being linked together parsimoniously through ancestral states was created (Templeton et al. 1992). The haplotypes within these maximum-step clades were joined in a network of steps, with a single mutation being one step. Inferred haplotypes were created to provide links between haplotypes more than one mutational step away. The haplotype networks were then grouped into nested clades using the method of Templeton et al. (1987). Proceeding always from the tips of the network tree, these tip clades were grouped with clades one mutational step away into a higher nesting clade, and then the next most internal clades grouped together, until all the clades were grouped into a higher nesting level. These clades were then grouped into higher clades, and so on, stopping at the last clade-step before the entire network would be encompassed by one clade. Ambiguities formed from loops in the network and odd numbers of clades were dealt with using coalescent theory (Crandall and Templeton 1993, Templeton and Sing 1993). Coalescent theory is used to break loops and to assign odd numbers of clades into nestingclades by a set of rules that assume that clades with more individuals are likely to be ancestral, and clades with few individuals (especially singletons (clades with a single individual)) are likely to be new mutations. Odd numbers of clades 
are grouped so that the odd clade is grouped with the clade with the least number of sampled individuals to increase the power in the geographical associations analysis.

\subsubsection{2 rooting the nested clade analysis network}

To root the $M$. campbelli nested clade diagrams we analysed all the individual sequences for COI and Total-mtDNA using TCS, instead of limiting the data to haplotypes. This outputs the probability for each haplotype that it is the root of the cladogram, worked out by considering the frequency of each haplotype and its position within the cladogram (see Castelloe and Templeton 1994). For the Total mtDNA cladogram we also viewed these outgroup weights in combination with the geographical coalescent method described in Crandall and Templeton (1993), which considers the size of the geographical area that each haplotype

was found over. We then compared the root suggested by NCA to the root suggested by phylogenetic analysis.

\subsubsection{3 nested clade analysis using geographical associations}

The program Geodis 2.0 (Posada et al., 2000) was used to analyse clade structure for geographical associations. The latitude and longitude were entered for each population and Geodis performed the calculations for distances within and between clades. The Geodis output was used in conjunction with the inference key (Nov 2001 version) provided with the program to estimate the history of population movement. A new key (25 Nov 2003: Templeton 2004) was also used to determine if there were any differences in the resulting population hypotheses. Clades were plotted onto a 
map of New Zealand for visual geographical analyses

\section{RESULTS}

\subsection{Collecting}

Maoricicada campbelli were collected from 70 populations throughout New Zealand (Table 3.1). Specimens were individually labelled with a 9-digit series: the last two digits of the year, the 2-letter standardised New Zealand district code from Crosby et al. (1998) a unique, usually informative, three-letter site code and a two-digit specimen code. For example, 02.MK.LOH.01 was the first specimen collected from the lower end of the ski road at Lake Ohau in the Mackenzie district in 2002. This allowed the use of the same code throughout the work, ease of identifying specimens from the same districts, years and sites, and minimised labelling errors. A two-letter country code (NZ) preceding the district code was also recorded, but is not necessary in this publication. In Table 3.1, districts are sorted roughly north-south (for district boundaries see Crosby et al, 1998). Populations are listed alphabetically within districts, and the GPS is the center of all individuals collected within one $\mathrm{km}$ of contiguous habitat. Only three of the 70 populations (MK.LOH, WD.HAR, CO.CRA) grouped individuals that were not collected from exactly the same place, and all were no greater than $1 \mathrm{~km}$ of contiguous habitat apart, and shared the same or similar haplotypes. Almost all individuals collected were males, females (identified in Appendix 3.4) were very difficult to find. M. campbelli were usually very abundant where found, however collecting conditions were not always conducive and populations collected in bad weather were represented by one or very few individuals. Male M. campbelli were usually easy to collect when 
singing, as they could be attracted to the collector by simulating the female response (wingflicks) to the male's song by snapping the fingers or clicking the tongue lightly. In bad weather M. campbelli were almost impossible to find, as they were well camouflaged to their rocky habitat and the males did not sing. One $M$. campbelli was collected in the rain by turning over rocks in a likely area.

Most of the M. campbelli populations that were additional to the sites of Buckley et al. (2001a) filled in gaps, rather than extending the sampled range of the species. The range of some clades, however, was extended by the sequencing of multiple individuals from some populations. No distant populations of $M$. campbelli were found in the North Island. Historical records from near Wellington remain undiscovered and represent either unfound or extinct populations, or possibly incorrect records of a similar species. The oral record listed in Fleming 1971 from Mt Tauhara, north of Mt Ruapehu, was most probably an incorrect report of the congeneric species Maoricicada iolanthe, which has an almost identical song and had not been reported from that location. In December 2001 we found only M. iolanthe from the base to the top of the mountain, however it is still possible that $M$. campbelli could be found in this location in later months. Further searching is needed to determine whether M. campbelli is definitely present or absent from Mt Tauhara. Previously unsampled populations in northwest Nelson and southern Otago were collected, which extended the sampled range of the species. Several interesting areas in the South Island containing M. campbelli were sampled after completion of these analyses, and will be used in further phylogenetic studies. 
Table 3.1 Maoricicada campbelli collecting localities (70 populations), GPS coordinates and haplotypes found in each population. Locations are sorted roughly north-south by district. District boundaries follow Crosby et al. 1998.

\begin{tabular}{|c|c|c|c|c|c|c|}
\hline Code +20 & $\begin{array}{l}\text { popula } \\
\text { umber }\end{array}$ & Location & ††GPS coordinates & col ${ }^{\mathrm{t}+}$ & $\begin{array}{l}\text { tDNA hap } \\
\text { A6-A8 }\end{array}$ & $\begin{array}{l}\text { plotypes } \\
\text { Total-mtDNA }\end{array}$ \\
\hline $\begin{array}{l}\text { aupo } \\
\text { TO.BRR } \\
\text { TO.MAN } \\
\text { TO.TSR }\end{array}$ & & $\begin{array}{l}\text { SH48 S of Whakapapa, W side of Mt Ruapehu } \\
\text { End of Mangatepopo road, W side of Mt Ruapehu } \\
\text { Tukino Skifield Road, off SH1, E side of Mt Ruapehu }\end{array}$ & $\begin{array}{l}\left(39^{\circ} 10.60^{\prime} \mathrm{S}, 175^{\circ} 30.75^{\prime} \mathrm{E}\right) \\
39^{\circ} 08.78^{\prime} \mathrm{S}, 175^{\circ} 34.85^{\prime}, \mathrm{E} \\
\left(39^{\circ} 17.78 \text { 'S, } 175^{\circ} 44.13^{\prime} \mathrm{E}\right)\end{array}$ & $\begin{array}{l}\text { A1 }(2) \\
\text { A1 } \\
\text { A1 } \\
\text { A3 } 3(1) \\
\text { A4 }\end{array}$ & $\begin{array}{l}\text { a1 (1) } \\
\text { a1 } 1 \text { (1) } \\
\text { a1 }(2) \\
\text { a2 }(1)\end{array}$ & $\begin{array}{l}\text { A1a1 (1) } \\
\text { A1a1 } 1) \\
\text { A1a1 (1) } \\
\text { A3a1 (1) } \\
\text { A4a2 }(1)\end{array}$ \\
\hline TO.WSF & [15] & Whakapapa Ski Field, W side of Mt Ruapehu & $\left(39^{\circ} 13.85^{\prime} \mathrm{S}, 175^{\circ} 33.60^{\prime} \mathrm{E}\right)$ & A2 (1) & a1 (1) & A2a1 (1) \\
\hline $\begin{array}{l}\text { Marlborough } \\
\text { MB.KOW } \\
\text { MB.KSR } \\
\text { MB.LSG } \\
\text { MB.LTT } \\
\text { (1) }\end{array}$ & {$\left[\begin{array}{l}31] \\
36] \\
41]\end{array}\right]$} & $\begin{array}{l}\text { Kowhai Scenic Reserve, off SH63, S side of Wairau R. } \\
\text { Kowhai Scenic Reserve, N side of Wairau River } \\
\text { Lake Sedgemere, off Rainbow-Wairau Divide Rd, } \\
\text { S tip of Lake Tennyson, off Rainbow-Wairau Divide Rd }\end{array}$ & $\begin{array}{l}\left(41^{\circ} 42.84 \text { 'S, } 173^{\circ} 06.76^{\prime} \mathrm{E}\right) \\
41^{\circ} 36.00^{\circ} \mathrm{S}, 173^{\circ} 22.20^{\prime} \mathrm{E} \\
\left(42^{\circ} 08.20^{\circ} \mathrm{S}, 172^{\circ} 54.75^{\prime} \mathrm{E}\right. \\
\left(42^{\circ} 12.80^{\prime} \mathrm{S}, 172^{\circ} 44.40^{\prime} \mathrm{E}\right)\end{array}$ & $\begin{array}{l}\text { BM2 (1) } \\
\text { BM1 (1) } \\
\text { BM1 (1) } \\
\text { BSA6 (1) } \\
\text { BSA7 (1) }\end{array}$ & $\begin{array}{l}b m 1(1) \\
b m 3(1) \\
b m 2(1) \\
b s a 6(1) \\
b s a 11(1)\end{array}$ & $\begin{array}{l}\text { BM2bm1 (1) } \\
\text { BM1bm3 } 1 \text { 1) } \\
\text { BM1bm2 (1) } \\
\text { BSA6bsa6 (1) } \\
\text { BSA7bsa11 }\end{array}$ \\
\hline MB.NST & [42] & Nocatchem Stream, Rainbow-Wairau Divide Rd & $\left(41^{\circ} 48.90^{\prime} \mathrm{S}, 172^{\circ} 54.85^{\prime} \mathrm{E}\right)$ & $\begin{array}{l}\mathrm{BM} 1(1) \\
\mathrm{BM} 2(1) \\
\mathrm{BM} 3(1)\end{array}$ & $\begin{array}{l}b m 1(2) \\
b m 5(1)\end{array}$ & $\begin{array}{l}\mathrm{BM} 1 b m 1 \text { (1) } \\
\mathrm{BM} 2 b m 5 \text { (1) } \\
\mathrm{BM} 3 b m 1(1)\end{array}$ \\
\hline MB.PVS & [13] & Pine Valley Stream, $\mathrm{N}$ side of Wairau river & $\left(41^{\circ} 32.17^{\prime} \mathrm{S}, 173^{\circ} 32.59^{\prime} \mathrm{E}\right)$ & $\mathrm{BM} 2(1)$ & $b m 1(2)$ & $\mathrm{BM} 2 b m 1\} 1)$ \\
\hline $\begin{array}{l}\text { MB.RFT } \\
\text { MB.SRW } \\
\text { MB.WRR }\end{array}$ & [35] & $\begin{array}{l}\text { Rag \& Famish Creek, Rainbow-Wairau Divide Rd } \\
\text { Serpentine Creek, Rainbow-Wairau Divide Rd } \\
\text { E of St Arnaud on SH63, just across Wairau River }\end{array}$ & $\begin{array}{l}\left(42^{\circ} 08.78^{\prime} \mathrm{S}, 172^{\circ} 51.08^{\prime} \mathrm{E}\right) \\
42^{\circ} 11.14^{\prime} \mathrm{S}, 172^{\circ} 46.82^{\prime}, \mathrm{E} \\
\left(41^{\circ} 43.21^{\prime} \mathrm{S}, 173^{\circ} 05.75^{\prime} \mathrm{E}\right)\end{array}$ & $\begin{array}{l}\text { BM5 }(1) \\
\text { BSA1 (1) } \\
\text { BM1 (1) } \\
\text { BM3 } 11 \\
\text { BM6 (1) }\end{array}$ & $\begin{array}{l}b m 2(1) \\
b s a 1(1) \\
b m 1(1) \\
b m 4(1) \\
b m 6(1)\end{array}$ & $\begin{array}{l}\text { BM5bm2 (1) } \\
\text { BSA1bsa1 (1) } \\
\text { BM1bm4 (1) } \\
\text { BM3bm1 (1) } \\
\text { BM6bm6 (1) }\end{array}$ \\
\hline $\begin{array}{l}\text { elson } \\
\text { NN.COB }\end{array}$ & & Cobb Reservoir, by dam & $\left(41^{\circ} 06.35^{\prime} \mathrm{S} 172^{\circ} 41.20^{\prime} \mathrm{E}\right)$ & $\begin{array}{l}\text { BN1 (3) } \\
\text { BN2 (1) } \\
\text { BN3 (1) }\end{array}$ & $\begin{array}{l}b n 1(4) \\
b n 2(1)\end{array}$ & $\begin{array}{l}\text { BN1bn1 (2) } \\
\text { BN1bn2 } 1) \\
\text { BN2bn1 } 1) \\
\text { BN3bn1 } 1 \text { (1) }\end{array}$ \\
\hline $\begin{array}{l}\text { aikoura } \\
\text { KA.ORO } \\
\text { KA.WAN }\end{array}$ & {$\left[\begin{array}{l}10 \\
11\end{array}\right]$} & $\begin{array}{l}\text { Oaro River, off SH1, S of Kaikoura } \\
\text { Wandle River, SH70, N of Waiau }\end{array}$ & $\begin{array}{l}\left(42^{\circ} 30.20^{\prime} \mathrm{S}, 173^{\circ} 29.15^{\prime} \mathrm{E}\right) \\
\left.42^{\circ} 30.56^{\prime} \mathrm{S}, 173^{\circ} 08.42^{\prime} \mathrm{E}\right)\end{array}$ & $\begin{array}{l}\text { BK1 }(1) \\
\text { BK2 (1) }\end{array}$ & $\begin{array}{l}b k 1(1) \\
b k 2(1)\end{array}$ & $\begin{array}{l}\text { BK1bk1 } 1 \text { (1) } \\
\text { BK2bk2 } 1)\end{array}$ \\
\hline
\end{tabular}




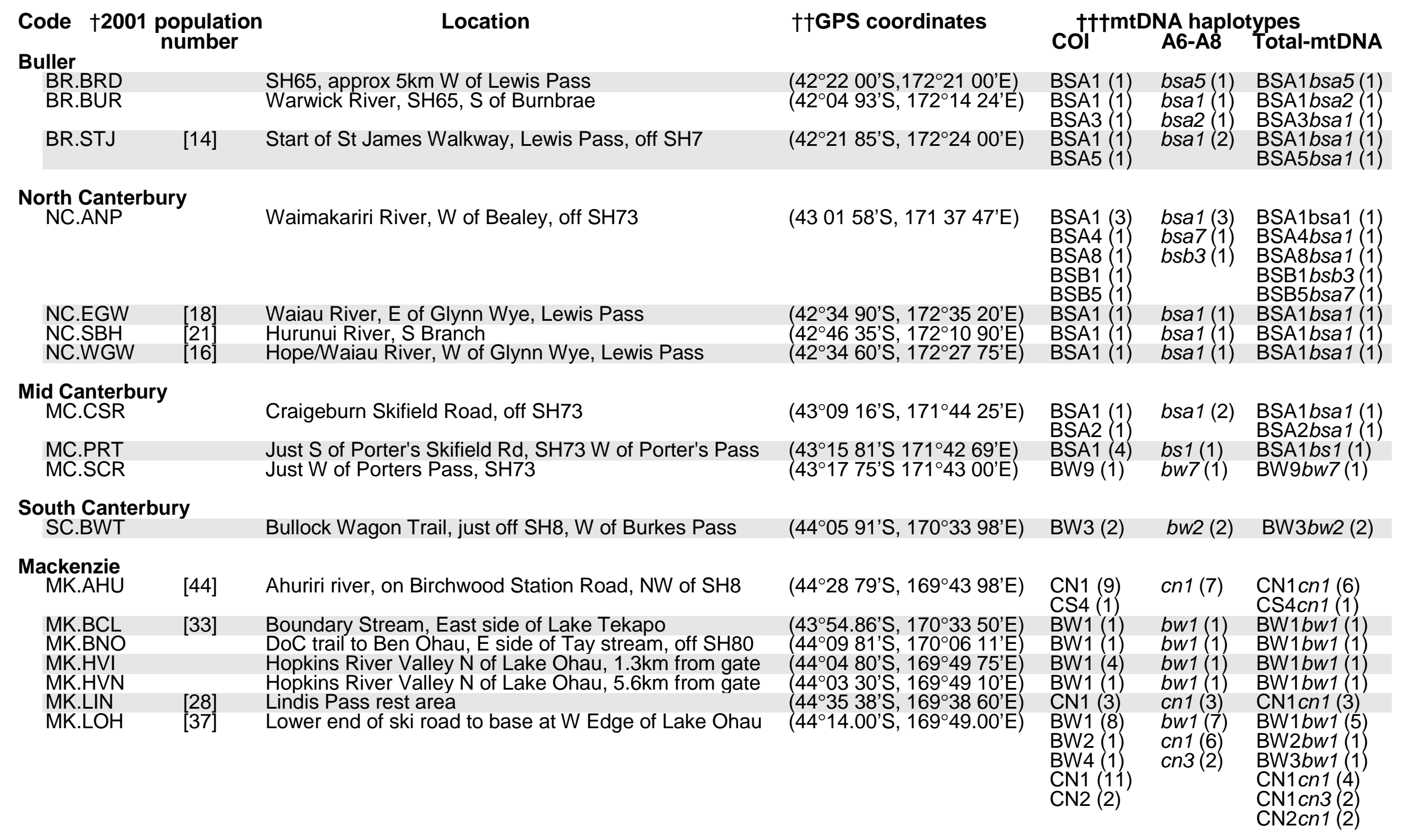


Code †2001 population

number

\section{Mackenzie continued}

MK.LTM

MK.MCA

MK.MCB

MK.MCR

MK.MCV

MK.OHA

$[23]$

Tasman Glacier 4WD track, Mt Cook National Park

SH80 to Mt Cook, N of SH8, W side of Lake Pukak

Birch Stream, Mt Cook Natl Park

SH80 to Mt Cook, S of Airport, W side of Tasman River

Mt Cook Visitor's Centre

Near top of Lake Ohau Skifield Rd

MK.RHD

Rhoborough Downs Rd at Twizel River, W of SH8

Arthurs Pass, Arthurs Pass National Park

Haast River, SH6, Just N of Haast Pass

WD.AR

WD.HAR

(1)

WD.OTR E side of Otira river, at bridge, $\mathrm{SH73}$

WD.PLC [40] Peg Leg Creek, Arthurs Pass National Park

\section{Fiordland}

FD.BST

FD.HCT

FD.HME

30]

South of Boundary Creek, SH94, N of Te Anau

\section{Central Otago}

\begin{tabular}{|c|c|c|c|}
\hline $\begin{array}{l}\text { CO.ABT } \\
\text { CO.AWR }\end{array}$ & {$[32]$} & $\begin{array}{l}\text { Albert Town, SE of Lake Wanaka } \\
\text { Awakino Ski Field Road, off SH83 near Kurow }\end{array}$ & $\left(\begin{array}{l}\left.44^{\circ} 40.80^{\prime} \mathrm{S}, 169^{\circ} 11.50^{\prime} \mathrm{E}\right) \\
\left(44^{\circ} 45.79^{\prime} \mathrm{S}, 170^{\circ} 21.62^{\prime} \mathrm{E}\right)\end{array}\right.$ \\
\hline CO.CRA & & Near Cardrona River, SH89, S of Cardrona & (445‥22'S, $\left.168^{\circ} 59.10^{\prime} E\right)$ \\
\hline $\begin{array}{l}\text { CO.KYE } \\
\text { CO.LTO } \\
\text { CO.OMR }\end{array}$ & {$\left[\begin{array}{l}29] \\
38 \\
24]\end{array}\right]$} & $\begin{array}{l}\text { Kye Burn River, S of Dansey's Pass } \\
\text { Little Omarama Stream, S of Omarama } \\
\text { Old Man Range, Symes Rd to Obelisk }\end{array}$ & $\begin{array}{l}\left(45^{\circ} 00.23^{\prime} \mathrm{S}, 170^{\circ} 16.45^{\prime} \mathrm{E}\right) \\
\left(44^{\circ} 35.85^{\prime} \mathrm{S}, 169^{\circ} 52.80^{\prime} \mathrm{E}\right) \\
\left(45^{\circ} 20.33^{\prime} \mathrm{S}, 196^{\circ} 15.26^{\prime} \mathrm{E}\right)\end{array}$ \\
\hline
\end{tabular}
$42^{\circ} 54.60^{\prime} \mathrm{S}, 171^{\circ} 33.60^{\prime} \mathrm{E}$
$44^{\circ} 05.90^{\prime} \mathrm{S}, 169^{\circ} 21.76^{\prime} \mathrm{E}$

††GPS coordinates

(4339.61'S, $\left.170^{\circ} 10.68^{\prime} \mathrm{E}\right)$ $44^{\circ} 06.00^{\prime} \mathrm{S}, 170^{\circ} 08.00^{\prime} \mathrm{E}$ $43^{\circ} 4430^{\prime} S, 170^{\circ} 0445^{\prime} \mathrm{E}$ $\left.43^{\circ} 44.11^{\prime} \mathrm{S}, 170^{\circ} 05.73^{\prime} \mathrm{E}\right)$
$\left(44^{\circ} 13.70^{\prime} \mathrm{S}, 169^{\circ} 46.85^{\prime} \mathrm{E}\right)$

$\left(44^{\circ} 11.83^{\prime} \mathrm{S}, 170^{\circ} 05.79^{\prime} \mathrm{E}\right)$ $\left.42^{\circ} 53.79^{\prime} \mathrm{S}, 171^{\circ} 33.56^{\prime} \mathrm{E}\right)$

(45⒙30'S, $167^{\circ} 47.75^{\prime} \mathrm{E}$ $45^{\circ} 14.40^{\prime} \mathrm{S}, 167^{\circ} 48.85^{\prime} \mathrm{E}$ (444‥97'S, $\left.167^{\circ} 59.35^{\prime} \mathrm{E}\right)$ (4348.68'S, $170^{\circ} 06.72^{\prime} \mathrm{E}$

$\left(42^{\circ} 51.20^{\prime} \mathrm{S}, 171^{\circ} 33.62^{\prime} \mathrm{E}\right)$ Old Man Range, Symes Rd to Obelisk
COI

t†tmtDNA haplotypes A6-A8 Total-mtDNA

BW1 1) bw1(1) BW1bw1 (1) BW1 1) bw1 1) BW1bw1 (1) BW1(1) bw1 1 BW1bw1 1) BW1 1) bw1 1) BW1bw1 1 BW1 (2) bw1 (1) BW1bw1 (1) BW1 (1) bw1(1) BW1bw1 1 CN1 (1) cn1(1) CN1cn1 (1) BW1 (11) bw1 (3) BW1bw1 (2) BW6 (1)

BSA2 (1) bsa1 (1) BSA2bsa1 (1) BSC1 (2) bsa1 (4) BSC1bsa1 (2) BSC4 (1) bsa10(1) BSC4bsa10

BSC5 (1) BSC6 (1) BSC6bsa1 (1) BSA1 (2) bsa3 (1) BSA1bsa3 (1) BSA2 (1) bsa1 (1) BSA2bsa1 (1)

BSC2 (1) $\quad$ bsa1 (1) BSC2bsa1 (1) $\begin{array}{lll}\text { BSC2 (2) } & \text { bsa1 1 } & \text { BSC2bsa1 (1) } \\ \text { BSC1 (2) } & \text { bsa1 (1) } & \text { BSC1bsa1 (1) }\end{array}$

\begin{tabular}{|c|c|c|}
\hline $\begin{array}{l}\text { CN1 (1) } \\
\text { BW1 (3) } \\
\text { BW2 (1) } \\
\text { BW7 } 1 \\
\text { BW8 (1) }\end{array}$ & $\begin{array}{l}c n 2(1) \\
b w 1(3) \\
b w 3(1) \\
b w 5(1) \\
b w 6(1)\end{array}$ & $\begin{array}{l}\text { CN1 cn2 (1) } \\
\text { BW1bw3 (2) } \\
\text { BW2bw3 } \\
\text { BW1 1 bw5 } \\
\text { BW7bw1 } \\
\text { BW8bw6 }\end{array}$ \\
\hline V1 (2) & cn1 (1) & CN1cn1 (1) \\
\hline $\begin{array}{l}\text { CN3 (1) } \\
\text { CN4 (1) }\end{array}$ & cn2 (2) & $\begin{array}{l}\text { CN3cn2 } \\
\text { CN4cn2 }\end{array}$ \\
\hline BW5 (1) & bw4 (1) & BW5bw4 (1) \\
\hline CN1 (1) & cn1 (1) & CN1cn1 (1) \\
\hline & cn1 (1) & CN1cn1 (1) \\
\hline & $\operatorname{cs} 1(1)$ & $\operatorname{CS} 3 \operatorname{cs} 1$ (1) \\
\hline
\end{tabular}




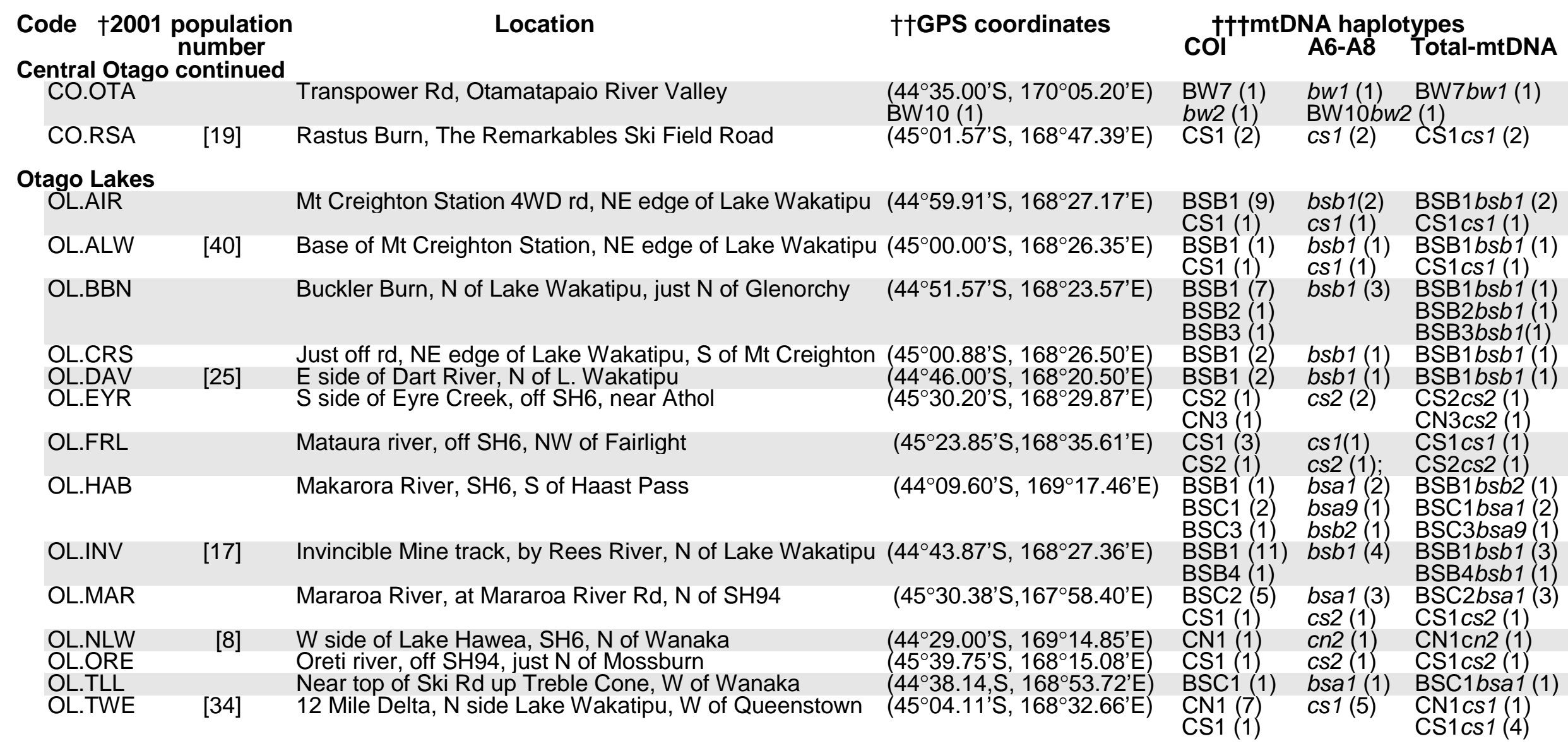

$\dagger \quad$ Numbers within square brackets refer to population numbers used by Buckley et al. 2001a. The GPS coordinates of these populations may differ from those published in Buckley et al. 2001a due to previous miscalculations.

†† GPS coordinates are given in Lat/Lon as degrees and minutes, using the NZGD49 grid.

t† Numbers in parentheses following haplotype refer to number of individuals in that haplotype for that population. 

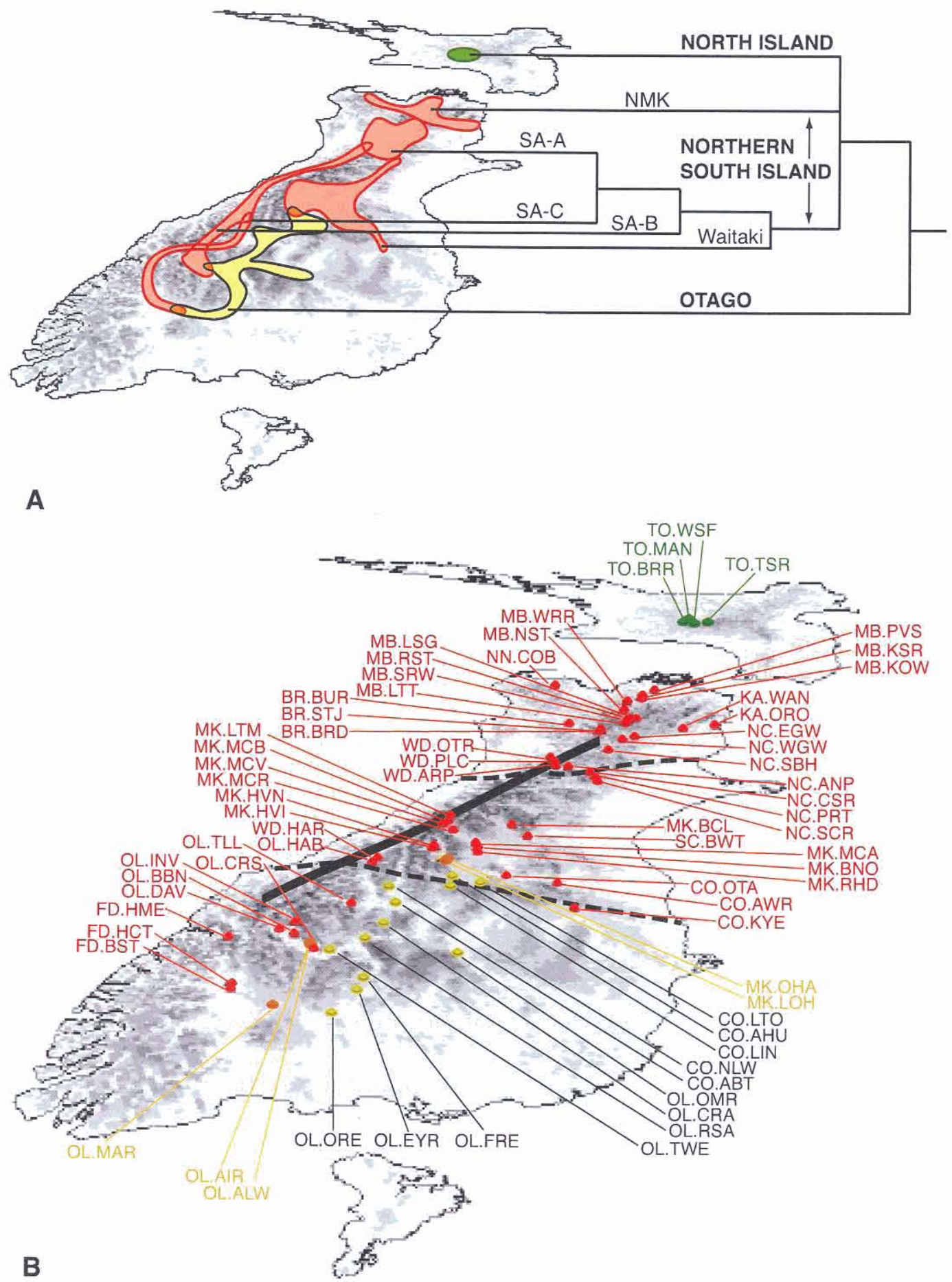

3.1 A. Map of NZ showing the location of the main mtDNA clades and subclades of Maoricicada campbelli. A consensus tree showing the phylogenetic relationships is superimposed. B. Map of NZ showing all $70 \mathrm{M}$. campbelli populations. See Table 3.1 for more information and GPS coordinates. The dark line indicates both the main backbone of the Southern Alps and part of the Alpine Fault. The area between the dashed lines indicates the central South Island biotic gap. For both A. and B., red = northern South Island clade, green = North Island clade, yellow $=$ Otago clade, orange indicates areas of secondary contact between the northern South Island and Otago clades. 3D DEM data provided by GeographX, NZ. NZ outline data provided by LINZ. Maps produced using ArcView 3D analyst (ESRI). 


\subsection{Molecular labwork: Sequencing and alignment}

Divergence levels were relatively low and there were no indels, therefore there was no alignment ambiguity. M. campbelli individuals were first sequenced for the $\mathrm{COI}$ gene region and aligned to determine their haplotype, then named according to phylogenetic and phylogeographic structure. For each $M$. campbelli with a unique $\mathrm{COI}$ sequence and for at least one individual from each population for each COI haplotype, an individual was sequenced for the A6-A8 region and assigned to a unique A6-A8 haplotype (Table 3.1, Appendix 3.4). Additional M. campbelli were sequenced in zones identified as potential contact areas between the northern South Island and Otago clades and from preliminary NCA results that indicated areas of particular interest. The following totals include the 35 M. campbelli sequenced by Buckley et al. (2001a). A total of $753 \mathrm{bp}$ of mtDNA from the 3 ' end of the COI region was sequenced for 223 M. campbelli, and 767 bp of the A6-A8 region, including the whole tRNA ${ }^{\text {Asp }}$ (64bp) and A8 (156bp) genes and 554bp of the 3' end of the A6 gene, were sequenced for 154 M. campbelli, for a total of 1520bp (Appendix 3.2) for 154 individuals (Appendix 3.4). The non-variable overlapping seven bases at the end of $A 8$ and the start of $A 6$ (found in other insect mtDNA, Buckley et al. 2001a) were included with $A 8$ for all analyses.

Haplotype names for COI (upper case) and A6-A8 (lower-case italics) were given an initial letter indicating the main clade to which they belonged (A: North Island, B: northern South Island, C: Otago) and subsequent letters and numbers indicating subclade and individual haplotype. The most common haplotypes were assigned first. Total-mtDNA haplotypes were written as a 
combination of $\mathrm{COI}$ and A6-A8 haplotype. Abbreviations for clades used throughout the text include: NI (North Island); northern-SI (northern South Island). The northern-SI clades include all South Island clades except Otago, specifically NMK (Nelson, Marlborough and Kaikoura), SA (Southern Alps, with subclades SA-A, SA-B, SA-C (Southern Alps A, etc)) and Waitaki. Appendix 3.2 shows an alignment of all 71 Total-mtDNA haplotypes found. All new sequences will be deposited in Genbank prior to these results being submitted for journal publication.

\subsection{Analysis results:}

\subsubsection{Sequences: base composition and sequence divergences}

The Chi square base composition test was negative $(15.66215, \mathrm{df}=216$, $p=1.0000$ ) indicating that no taxa had significantly different composition of bases compared to the rest. From 1520 bases sequenced there were 115 parsimony informative sites found (Table 3.2). Most of the differences occurred in the third base positions, with only $7.4 \%$ of amino acids being variable. The most variable partition (in accordance with other insects (Simon et al. 1994)) was the $\mathrm{A} 8$ region (Table 3.2).

Appendix 3.3 shows the uncorrected and corrected molecular distances between all Total-mtDNA haplotypes, which are summed up in Table 3.3. Corrected distances were estimated using the $\mathrm{ML} T r N+I$ model parameters. The highest sequence divergences are between the Otago haplotypes and all others, as reported by Buckley et al. (2001a), with a maximum of 0.045 
Table 3.2 Maoricicada campbelli mtDNA variation. All values are calculated with the seven overlapping bases at the 3' and 5' end of A8 and A6 respectively included with A8. Gamma values estimated using the method of Frati et al. 1997. Maximum-step clades estimated using TCS 1.13.

\begin{tabular}{|c|c|c|c|c|c|c|c|c|c|}
\hline 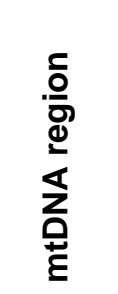 & 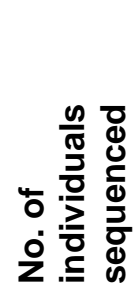 & 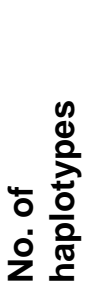 & 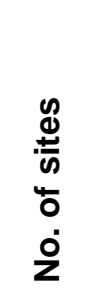 & 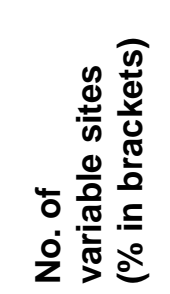 & 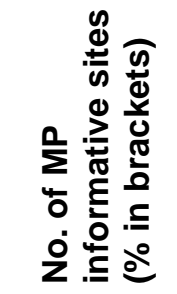 & 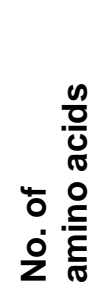 & 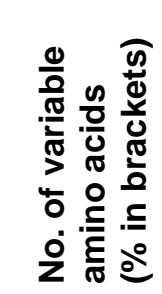 & 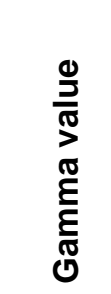 & 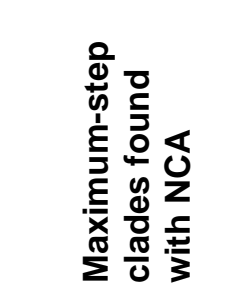 \\
\hline DI $1^{\text {st }}$ & 223 & - & 251 & $\begin{array}{ll}7 & (2.8)\end{array}$ & $4 \quad(1.6)$ & - & - & * & - \\
\hline $2^{n}$ & 223 & - & 251 & $3(1.2)$ & $0 \quad(0.0)$ & - & - & * & - \\
\hline $13^{r}$ & 223 & - & 251 & 60 (23.9) & $39(15.5)$ & - & - & 2.773 & - \\
\hline all & 223 & 52 & 753 & $70 \quad(9.3)$ & $43 \quad(5.7)$ & 251 & (3.6) & 0.002 & $\mathrm{NI}, \mathrm{NSI}$, Otago \\
\hline $\mathrm{A}^{\mathrm{A}}$ & 154 & 3 & 64 & $2(3.1)$ & 1 (1.6) & - & - & 0.014 & $\mathrm{NI}+\mathrm{NSI}+\mathrm{Otagc}$ \\
\hline & 154 & - & 52 & $7(13.5)$ & $\begin{array}{ll}4 & (7.7) \\
\end{array}$ & - & - & * & - \\
\hline & 154 & - & 52 & $5(9.6)$ & $4 \quad(7.7)$ & - & - & * & - \\
\hline & 154 & - & 52 & $7(13.5)$ & $6(11.5)$ & - & - & * & - \\
\hline & 154 & 16 & 156 & 19 (12.2) & $14(9.0)$ & 52 & $13(25.0)$ & 0.647 & $\mathrm{NI}+\mathrm{NSI}$, Otagc \\
\hline & 154 & - & 182 & $13 \quad(7.1)$ & $\begin{array}{ll}9 & (4.9)\end{array}$ & - & - & 0.019 & - \\
\hline & 154 & - & 183 & 4 (2.2) & $1 \quad(0.5)$ & - & - & * & - \\
\hline & 154 & - & 182 & $43(23.6)$ & 37 (20.3) & - & - & * & - \\
\hline & 154 & 31 & 547 & $60(11.0)$ & $47 \quad(8.6)$ & 181 & $12(6.6)$ & 0.000 & NI, NSI, Otago \\
\hline & 154 & 71 & 1520 & $151 \quad(9.9)$ & $105 \quad(6.9)$ & 484 & $(7.0)$ & 0.011 & $\mathrm{NI}, \mathrm{NSI}$, Otago \\
\hline
\end{tabular}

* indicates gamma values too large to be estimated.

$\mathrm{Nl}=$ North Island, $\mathrm{NSI}=$ northern South Island.

Table 3.3 Summary of mtDNA distances shown in Appendix 3.3 from 71 Total-mtDNA Maoricicada campbelli haplotypes. Uncorrected distances are shown above distances corrected using $\mathrm{ML}(\mathrm{TrN}+\mathrm{I}$ model).

\begin{tabular}{|c|c|c|c|c|c|c|c|c|}
\hline \multicolumn{4}{|c|}{$\begin{array}{l}\text { Within and between } \\
\text { Total-mtDNA distances } \\
\text { for two main clades }\end{array}$} & \multicolumn{5}{|c|}{$\begin{array}{l}\text { Within and between } \\
\text { Total-mtDNA distances } \\
\text { for three main clades }\end{array}$} \\
\hline & \multicolumn{2}{|c|}{$\begin{array}{c}\mathrm{NI+} \\
\text { northern-SI }\end{array}$} & Otago & & NI & \multicolumn{2}{|c|}{ northern-SI } & Otago \\
\hline $\begin{array}{c}\mathrm{NI+} \\
\text { northern-SI }\end{array}$ & \multicolumn{2}{|c|}{\begin{tabular}{|l|}
$0.001-0.028$ \\
$0.001-0.033$
\end{tabular}} & $\begin{array}{l}0.037-0.045 \\
0.046-0.062\end{array}$ & NI & \begin{tabular}{|l|}
$0.001-0.003$ \\
$0.001-0.003$ \\
\end{tabular} & \multicolumn{2}{|c|}{\begin{tabular}{|c|}
$0.018-0.028$ \\
$0.020-0.033$
\end{tabular}} & $\begin{array}{l}0.039-0.043 \\
0.051-0.058\end{array}$ \\
\hline Otago & & & \begin{tabular}{|l|}
$0.001-0.007$ \\
$0.001-0.008$
\end{tabular} & northern-SI & & \multicolumn{2}{|c|}{\begin{tabular}{|c|}
$0.001-0.018$ \\
$0.001-0.020$
\end{tabular}} & $\begin{array}{l}0.037-0.045 \\
0.046-0.062\end{array}$ \\
\hline \multicolumn{9}{|c|}{ Within-clade Total-mtDNA distances for subclades } \\
\hline \multicolumn{2}{|c|}{ NMK } & \multicolumn{2}{|c|}{ SA } & Waitaki & \multicolumn{2}{|c|}{ Otago north } & \multicolumn{2}{|c|}{ Otago south } \\
\hline \multirow{2}{*}{\multicolumn{2}{|c|}{$\begin{array}{l}0.001-0.008 \\
0.001-0.008\end{array}$}} & \multirow{2}{*}{\multicolumn{2}{|c|}{$\begin{array}{l}0.001-0.007 \\
0.001-0.007\end{array}$}} & 0.00 & \multirow{2}{*}{\multicolumn{2}{|c|}{$0.001-0.004$}} & \multirow{2}{*}{\multicolumn{2}{|c|}{$\begin{array}{c}0.001-0.004 \\
0.001-0.004\end{array}$}} \\
\hline & & & & $0.001-0.011$ & & & & \\
\hline
\end{tabular}


uncorrected or 0.062 corrected expected number of substitutions per site (genetic distances). This is high for within-species divergence in insects in general, and for other closely related cicadas (Buckley et al. 2001a,b). Many other Maoricicada species that are well-differentiated in song, habitat and morphology are much less distant in mtDNA (Buckley et al. 2001b). The sequence divergence between $\mathrm{NI}$ and northern-SI is also relatively high at a maximum of 0.028 uncorrected, 0.033 corrected expected number of substitutions per site. Sequence divergence is very low within all clades except the northern-SI, which has a maximum distance of 0.018 uncorrected, 0.020 corrected expected number of substitutions per site. The northern-SI clade had by far the greatest number of haplotypes and the deepest within-clade structuring.

\subsubsection{Phylogenetic results (ML and Bayesian)}

A heuristic ML search found one tree, with a -In likelihood of 3642.5, while the best Bayesian tree found had a -In likelihood of 3603.2. The Bayesian analysis was found to be stationary with a 10\% burn-in (the first 4000 trees), and no autocorrelation was detected between samples. The position of the deep Otago clade as sister to and significantly different from all other M. campbelli clades is the strongest phylogenetic result in both the ML and Bayesian analyses (Fig 3.2). This again reinforces the data analysis from Buckley et al. (2001a) that indicated that the Otago clade was different enough from all other M. campbelli to possibly be a separate species. A small song difference in the Otago M. campbelli, discovered while sampling (unpublished data), is being investigated to explore this possibility. 


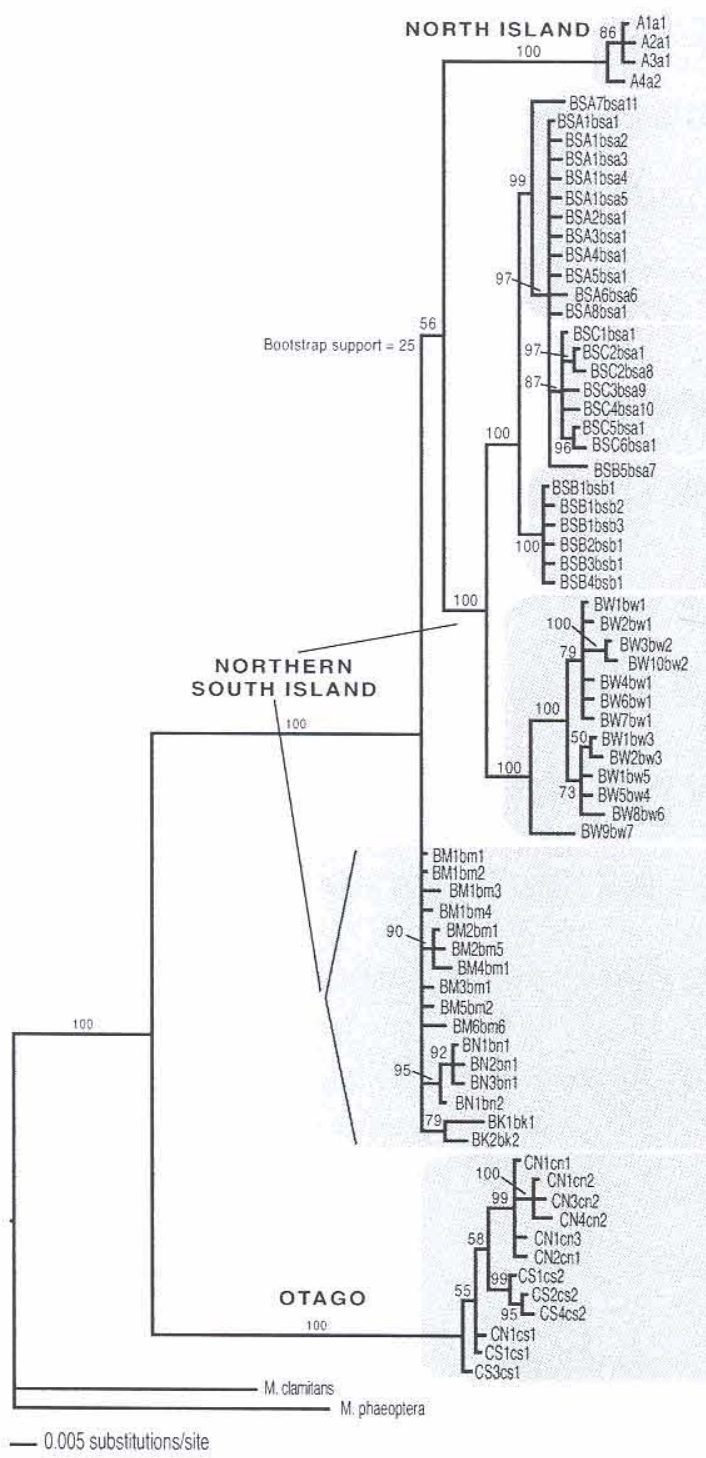

A.
NI

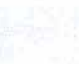

SA-A

SA-C

$S A-B$

Otago

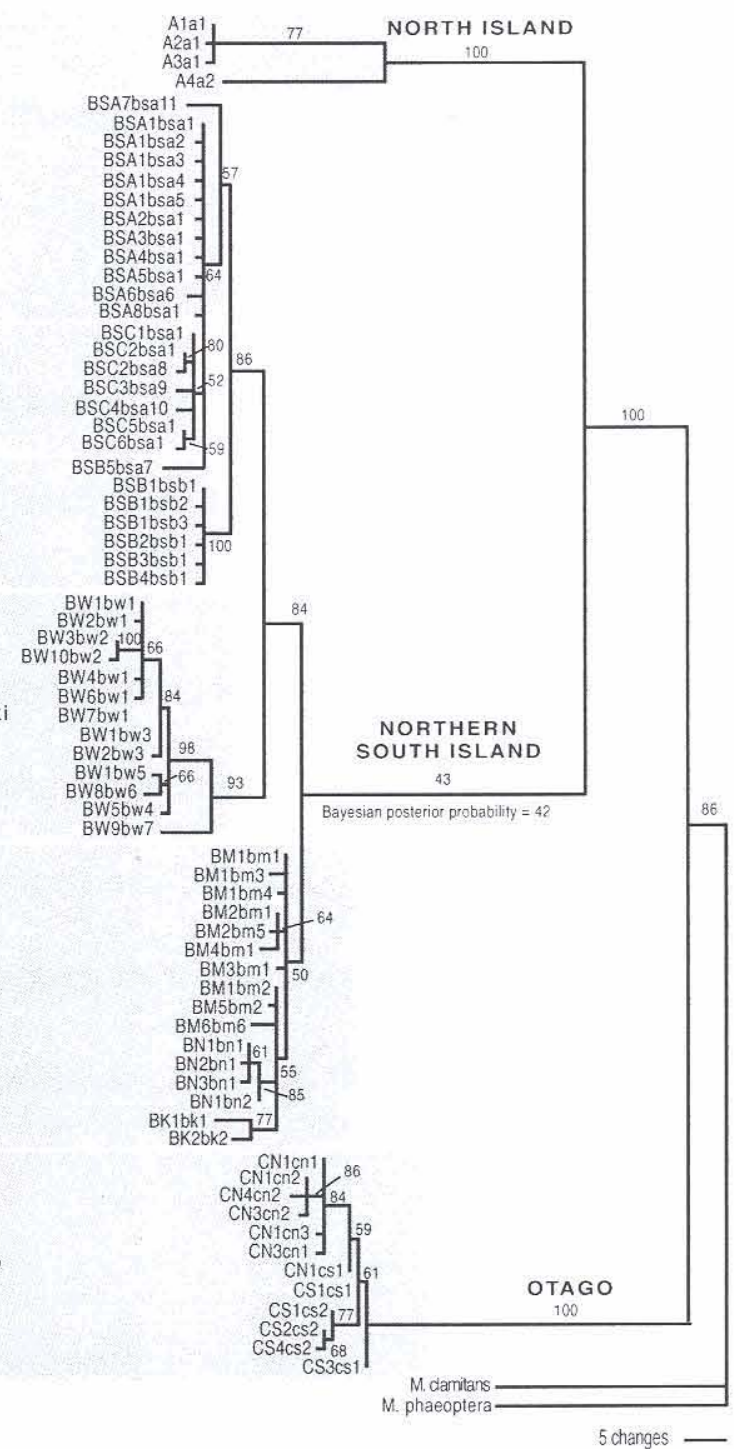

B.

Figure 3.2 Phylograms for 71 Maoricicada campbelli Total-mtDNA (COI+A6-A8) haplotypes. A. Bayesian topology and branch lengths estimated from a partitioned-model with 4 million generations and a 10\% burn-in. Numbers next to nodes represent the Bayesian posterior probabilities. B. Maximum likelihood topology and branch lengths estimated from a $\mathrm{TrN}+\mathrm{I}$ model. Numbers next to nodes represent bootstrap values estimated from 44 pseudoreplicates. Grey highlighting indicates clades found with nested clade analysis, which are not necessarily monophyletic. Names in bold capitals represent the three main clades found in NCA analyses. $\mathrm{NI}=$ North Island, SA = Southern Alps, NMK = Nelson, Marlborough and Kaikoura. 
The position of the $\mathrm{NI}$ clade is ambiguous, and it is possible that this clade is sister to either the NMK clade or the Waitaki+SA clades. Most likely, all three clades split very close in time; neither analysis provides strong support for their respective resolutions (Fig 3.2). The node that separates the $\mathrm{NI}+$ Otago and northern-SI clades was only represented in $43 \%$ of bootstrap pseudoreplicates (Fig 3.2). This same node was found in $51 \%$ of $200 \mathrm{ML}$ and $98 \%$ of $500 \mathrm{MP}$ bootstrap pseudoreplicates of a smaller dataset in Buckley et al. 2001a. The Bayesian consensus tree differed in only one significant way from the $M L$ tree; in the placement of this $\mathrm{NI}$ clade, either as sister to or branching off from within the northern-SI clade (Fig 3.2). The Bayesian analysis showed greater support for the formation of the NI clade after both the NMK and Otago clades had split off $(58 \%)$ rather than a split with the whole northern-SI clade $(42 \%)$. These low posterior probabilities indicate significant ambiguity in the data. ML bootstrap support for the most likely Bayesian result with Otago+NMK sister to $\mathrm{NI}+\mathrm{SA}+$ Waitaki was only $25 \%$.

Most other nodes are reasonably well supported in both $\mathrm{ML}$ and Bayesian analyses (Fig 3.2). The five major clades found by Buckley et al. (2001a) are all represented in these further analyses, some with additional support from the greater number of haplotypes found. Increased individual/population sampling has resulted in greater ambiguity of the placement of the NI clade, as being either within or sister to the northern-SI clades. Additionally, the ML tree shows only $50 \%$ bootstrap support for a monophyletic NMK and the Bayesian tree shows this group as a paraphyletic assemblage that is sister to the remaining non-Otago clades. The increased sampling in the Southern Alps area led to the 
SA clade being split into three subclades (SA-A, SA-B, SA-C) (Figs 3.1, 3.2). From the phylogenetic analyses, the SA-B clade is sister to the SA-A+SA-C clades, with Waitaki sister to all SA clades (Fig 3.2). The clades SA-A and SA$B$ found in the nested clade analyses are not monophyletic in the phylogenetic Bayesian and $\mathrm{ML}$ analyses. One haplotype (BSA7bsa11) is sister to all the other SA-A and SA-C haplotypes in both the Bayesian and ML analyses, but is found within the SA-A clade in the nested clade analysis. The haplotype (BSB5bsa7) links the SA-B clade to the SA-A clade in the nested clade analysis (Fig 3.4), but in the phylogenetic analyses this haplotype is part of the main cluster of SA-A haplotypes.

\subsubsection{Phylogeographic nested clade analysis results}

\subsubsection{Nested clade analysis network diagrams}

Networks were calculated for COI, tRNA ${ }^{\text {Asp }}, A 6$ and $A 8$ and the Total mtDNA haplotypes using TCS 1.13. In all NCA haplotype networks except for A8 and tRNA $^{\text {Asp }}$, haplotypes from the NI, northern-SI and Otago clades were separated by a greater number of steps than the $95 \%$ parsimony limit (Templeton 1992), which meant NCA could only be performed within each of these major clades (Table 3.2, Figs 3.3, 3.4). For A8, two major clades were found, one including $\mathrm{NI}+$ northern-SI, and the other Otago (Table 3.2). For tRNA ${ }^{\text {Asp }}$, all haplotypes were joined together into a single clade (Table 3.2). The small number of individuals sequenced, of variable sites and of haplotypes resulted in the individual NCA analyses of tRNA ${ }^{A s p}, A 8$ and $A 6$ being precluded from further analysis. COI (greater number of individuals) and Total-mtDNA (greater number of haplotypes) NCA networks were analysed further (Figs 3.3, 3.4). 
The COI and Total-mtDNA cladograms produced by TCS included ambiguous linkages (Figs 3.3, 3.4). Templeton and Sing (1993) give four possible reasons for recovering ambiguities that reflect data conflict: recombination, scoring errors, incomplete scoring and homoplasy. Mitochondrial DNA, however, has rarely been documented to undergo recombination (Rokas et al. 2003) and all DNA sequences were complete and were checked extensively for possible recording errors. The ambiguities recovered from all the TCS cladogram analyses are therefore probably due to homoplasy, where one or more of the mutational sites have undergone further mutations resulting in the loss of data. Additional sequencing of $M$. campbelli would be useful, especially from populations found in geographical areas between clades with currently ambiguous assignments. It is probable that new haplotypes would be found, and some of these may be haplotypes that could make associations between clades clearer.

COI nested clade diagram ambiguities: For the COI cladogram (Fig 3.3), within clade 4-1 (northern South Island) there were two loops leading to ambiguous decisions on associations between clades. The inferred haplotypes in clade 1-Y could have been grouped with clade 2-1, (SA-A), which would have resulted in a single third-level clade grouping everything together. However, coalescent rules state that in cases where ambiguities are caused by odd numbers of clades that the stranded group be grouped with the clade that has the fewest members, thus maximizing the power of the geographical analysis. This meant that clade 1-Y grouped with the Marlborough clade, which in the 

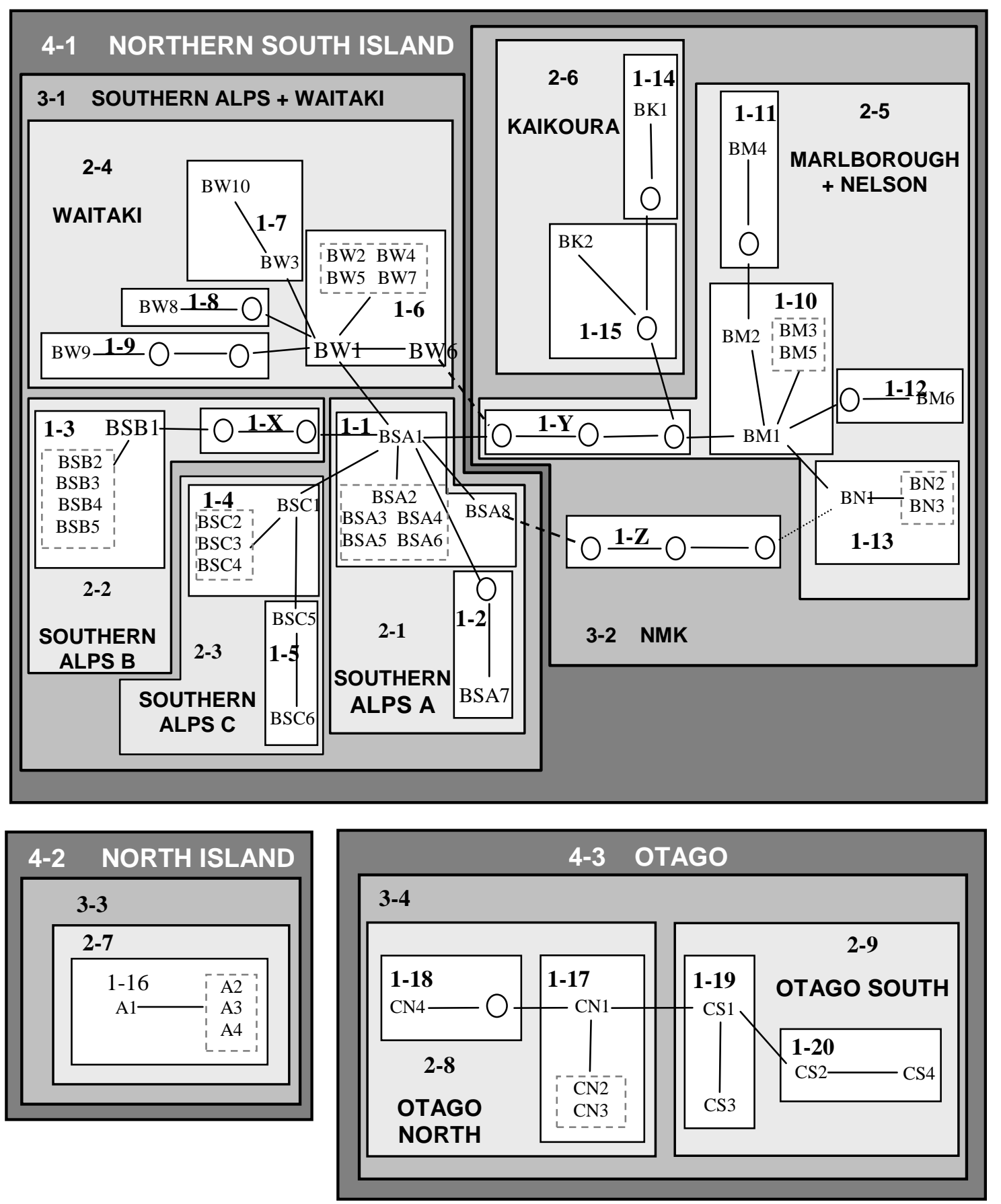

Figure 3.3 Nested cladogram of 52 haplotypes of Maoricicada campbelli calculated by TCS from 753 bp of the COI mtDNA gene region. The 95\% parsimony connection limit = 11 steps. Names represent unique haplotypes, circles represent inferred haplotypes. Straight lines between haplotypes indicate one mutational step. Dashed lines represent alternative connections that were disregarded using information from coalescent theory. Dashed boxes around haplotype names indicate unique tip haplotypes, each one step removed from the interior haplotype. Clade numbers are given as $\mathrm{Y}-\mathrm{X}$, with $\mathrm{Y}=$ number of the clade level, $\mathrm{X}=$ unique number within that level. 
next nesting level grouped Kaikoura with the Marlborough clade. Since Kaikoura is more closely related to the Marlborough haplotypes in the phylogenetic analyses, and has fewer mutational steps to the Marlborough clade than any other, this assignment is satisfying. Clade $1-Y$ could be parsimoniously joined to clade 1-6 through haplotype BW6, and Clade 1-Z could be joined to clade 1-1 through haplotype BSA8. Haplotypes BSA8 and BW6 are each singletons (clades represented by only one individual), which makes them much more likely to be tip clades than internal clades. This means that neither of these associations are viable, and we can dispense with clade 1$Z$ altogether. To add additional support to this, haplotypes BSA1 and BM1 are both internal clades represented by many individuals, and are much more likely to link the two major clades (3-1 SA+Waitaki and 3-2 NMK) together.

Total-mtDNA nested clade diagram ambiguities: The main ambiguity within the Total-mtDNA diagram (Fig 3.4) is in the connection between the NMK and SA groups. The SA clades could be joined to the rest of the northern-SI clade through either the SA-A or SA-B clades. The phylogenetic trees indicate that SA-B split from Marlborough and Waitaki at the same time as the ancestor to SA-A and SA-C (Fig 3.2). NCA of COI indicates that SA-A is an interior clade (linking to NMK and Waitaki), and SA-B evolved from this (Fig 3.3). However, in the Total-mtDNA diagram, the linking to SA-B through the interior haplotype BSB1bsb1 makes more sense than linking to SA-A through the inferred haplotype attached to the singleton BSA6bsa6. As stated above, it is more likely that an interior clade, rather than a singleton, is linked to other clades. In this case the singleton is not the linking haplotype, but the inferred haplotype 


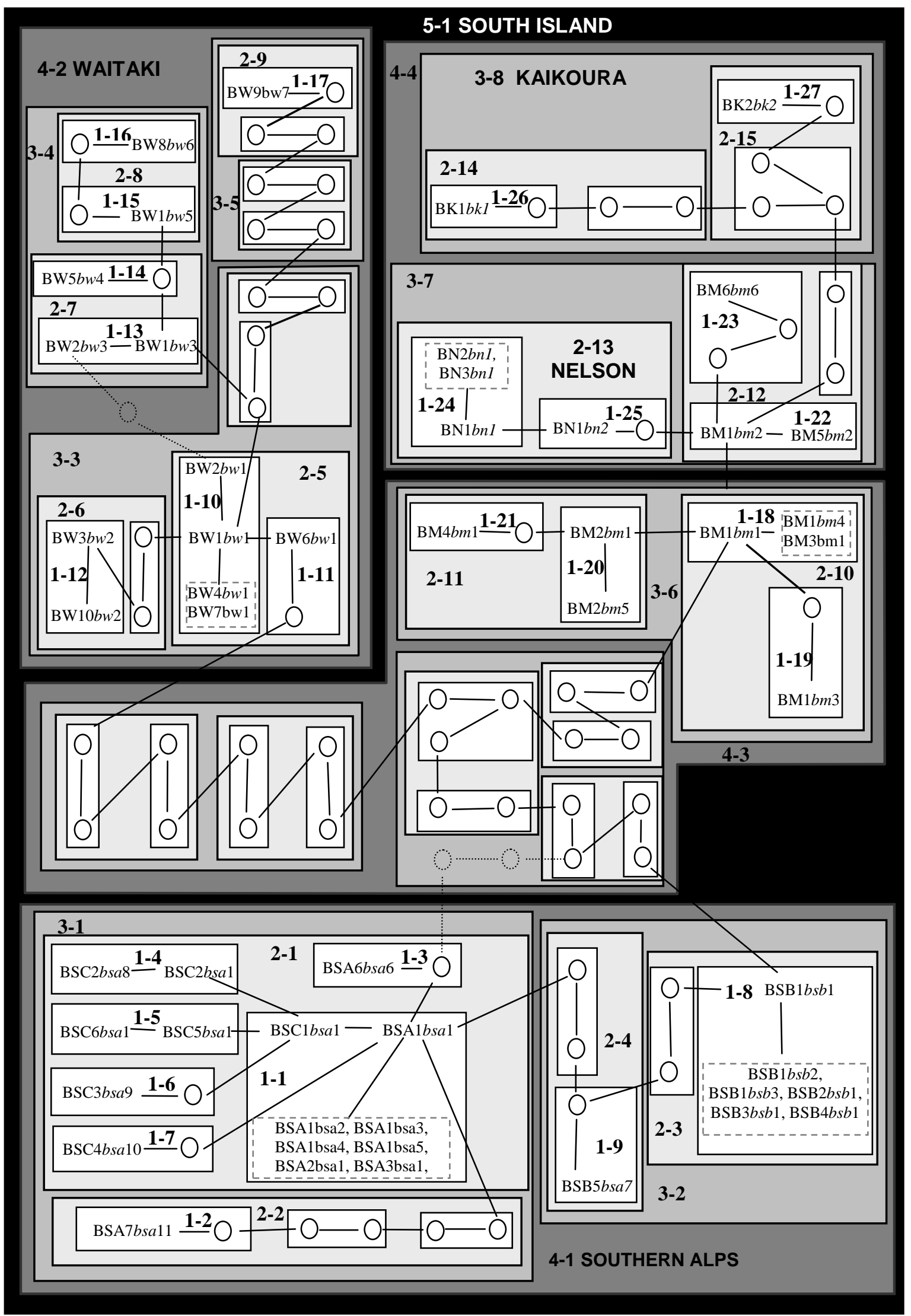



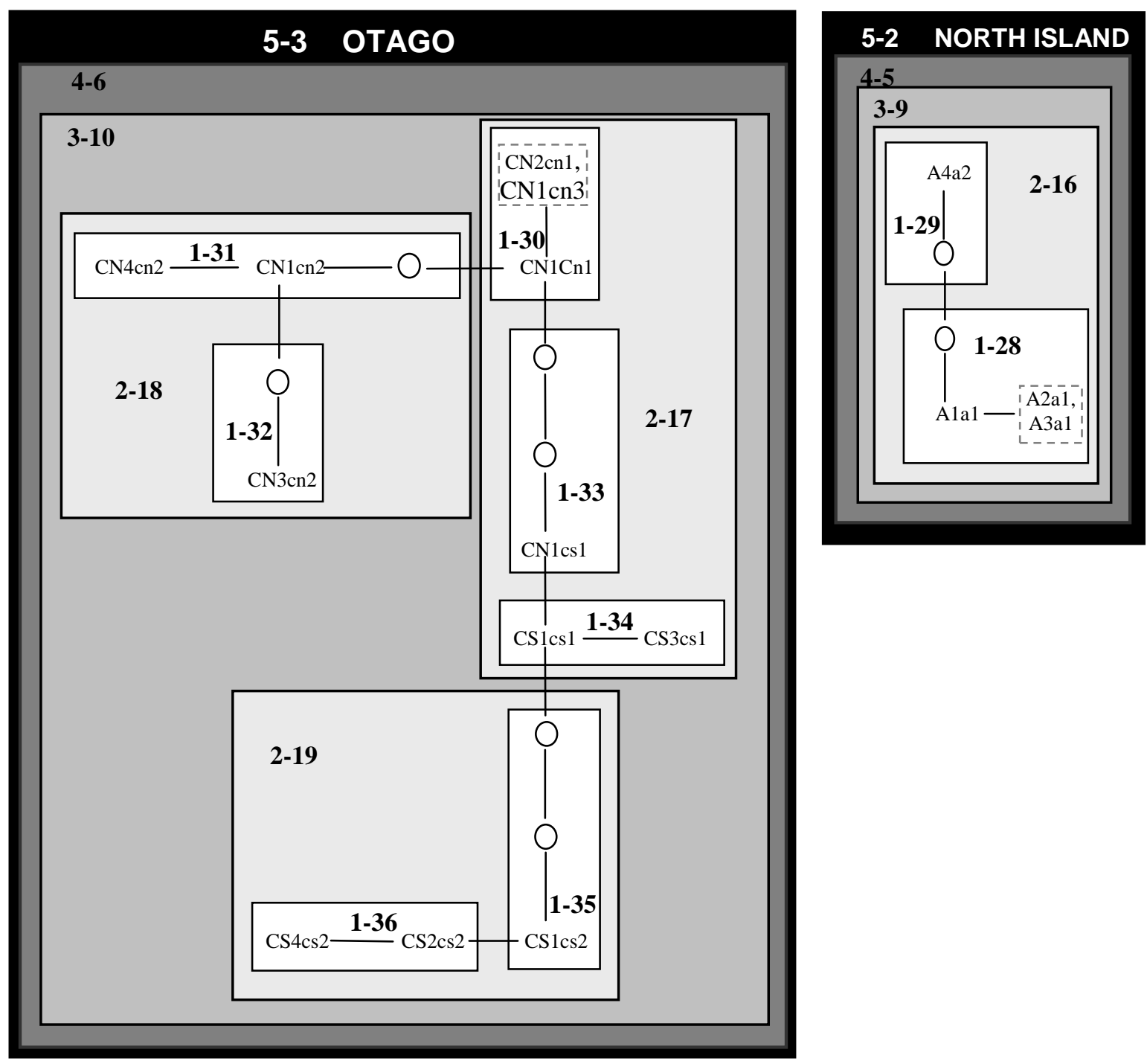

Figure 3.4 Nested cladogram of 71 haplotypes of Maoricicada campbelli calculated by TCS from $1520 \mathrm{bp}$ of the COI and A6-A8 mtDNA gene regions. The 95\% parsimony connection limit $=17$ steps. Names represent unique haplotypes, circles represent inferred haplotypes. Straight lines between haplotypes indicate one mutational step. Dashed lines represent alternative connections that were disregarded using information from coalescent theory. Dashed boxes around haplotype names indicate unique tip haplotypes, each one step removed from the interior haplotype. Clade numbers are given as $\mathrm{Y}-\mathrm{X}$, with $\mathrm{Y}=$ number of the clade level, $\mathrm{X}=$ unique number within that level. 
attached to it is. There is still some ambiguity in this decision, which can be cleared up by examining the higher nesting structure. If the NMK and Waitaki haplotypes are joined to the SA clades via the SA-A clade, the SA-A haplotype BSA6bsa6 remains nested with the inferred haplotypes linking it to the NMK clade. This persists to the fourth nesting level, linking BSA6bsa6 to part of the Marlborough clade. This is unsatisfactory, because this individual is south of Island Saddle and in the SA-A territory (Fig 3.6). Island Saddle separates all other SA-A and Marlborough haplotypes. In addition to the geographical analysis, BSA6bsa6 is only two steps removed from the closest SA-A haplotype, yet 13 steps removed from the closest Marlborough haplotype.

The only other ambiguity in the Total-mtDNA diagram is in the linking of haplotypes in the Waitaki clade (Fig 3.4). It is much more likely that BW1bw1 links through an inferred haplotype to BW1bw3, than the singletons BW2bw1 and BW2bw3 link the clades together.

\subsubsection{Nested clade diagram rooting}

For the COI cladogram, the haplotypes with the highest outgroup probabilities calculated with TCS were: northern-SI clade (BSA1; outgroup weight 0.19, closest other haplotype (BW1) 0.18; NMK haplotypes 0.00-0.03), Otago clade (CN1; outgroup weight 0.41 , closest other haplotype (CS1) 0.41 but fewer connections) and $\mathrm{NI}$ clade (A1; outgroup weight $0.88,0.04$ for all other haplotypes). 
For the Total-mtDNA cladogram, the haplotypes with the highest outgroup probabilities calculated with TCS were: northern-SI clade (BSA1bsa1; outgroup weight 0.14 , closest other haplotype (Bw1bw1) 0.12; NMK haplotypes 0.00 0.04), Otago clade ( $\mathrm{CN} 1 \mathrm{cn} 1$; outgroup weight 0.36 , closest other haplotype (Cs1cs1) 0.18) and NI clade (A1a1; 0.77, all other haplotypes 0.08$)$. Viewing the geographical ranges finds that in most cases the haplotype with the greatest outgroup weight from the TCS analysis is also the haplotype with the largest geographical range in each clade, which offers further support for the chosen haplotype to be the correct outgroup. In one instance, a different haplotype was found in more populations (BW1bw1 was found in more populations than BSA1bsa1), however this was due to uneven sampling, and the total area covered by the two haplotypes is similar.

The nested cladogram rooting suggested by the coalescent methods differed in every clade to that suggested by the phylogenetic analyses. In the haplotyperich clades Otago and northern-SI there were several haplotypes (and in the northern-SI clade several subclades) that split off before the haplotype suggested by the coalescent methods as the root. There was not always strong unambiguous support to determine the oldest-branching haplotypes in the phylogenetic trees, however these analyses were able to use information from other clades and outgroups that were too far away to be included in the coalescent methods. 


\subsubsection{Nested clade analysis using geographical associations}

Buckley et al. (2001a) found that all clades were geographically well defined. The additional sampling from this study supports this, with some clades being separated by only a few km, or sympatric in narrow contact zones (Figs 3.1, 3.6). Additional populations sampled within the published geographical range of any of the clades were always associated with the encompassing clade. New populations extending clade boundaries always extended the range of the predicted clade, with the only uncertain element being in the precise location of zones of contact. The new population from the Nelson area was most closely related to the geographically proximate Marlborough clade, as predicted. The additional populations in southern Otago were from either the Otago clade or the SA-C clade, also as predicted.

The Southern Alps clade was split into three smaller clades, each with a unique geographic range. SA-B, which is sister to SA-A and SA-C, spans the central Southern Alps, while SA-A and SA-C are found at the northern and southern ends of the Southern Alps respectively (Figs 3.1, 3.6). SA-B contacts both other SA clades at their extremes. The Waitaki clade covers much the same area found in the Buckley et al. 2001a sampling, however one haplotype belonging to this clade (BW9bw7) was found much farther north (near the Craigeburn range, just South of a SA-A population), bridging an unsampled area in the central South Island (Figs 3.1, 3.6).

The historical population movements estimated by the Geodis analyses of $\mathrm{COI}$ and Total-mtDNA clades are summarised in Tables 3.4 and 3.5. Appendix 3.5 


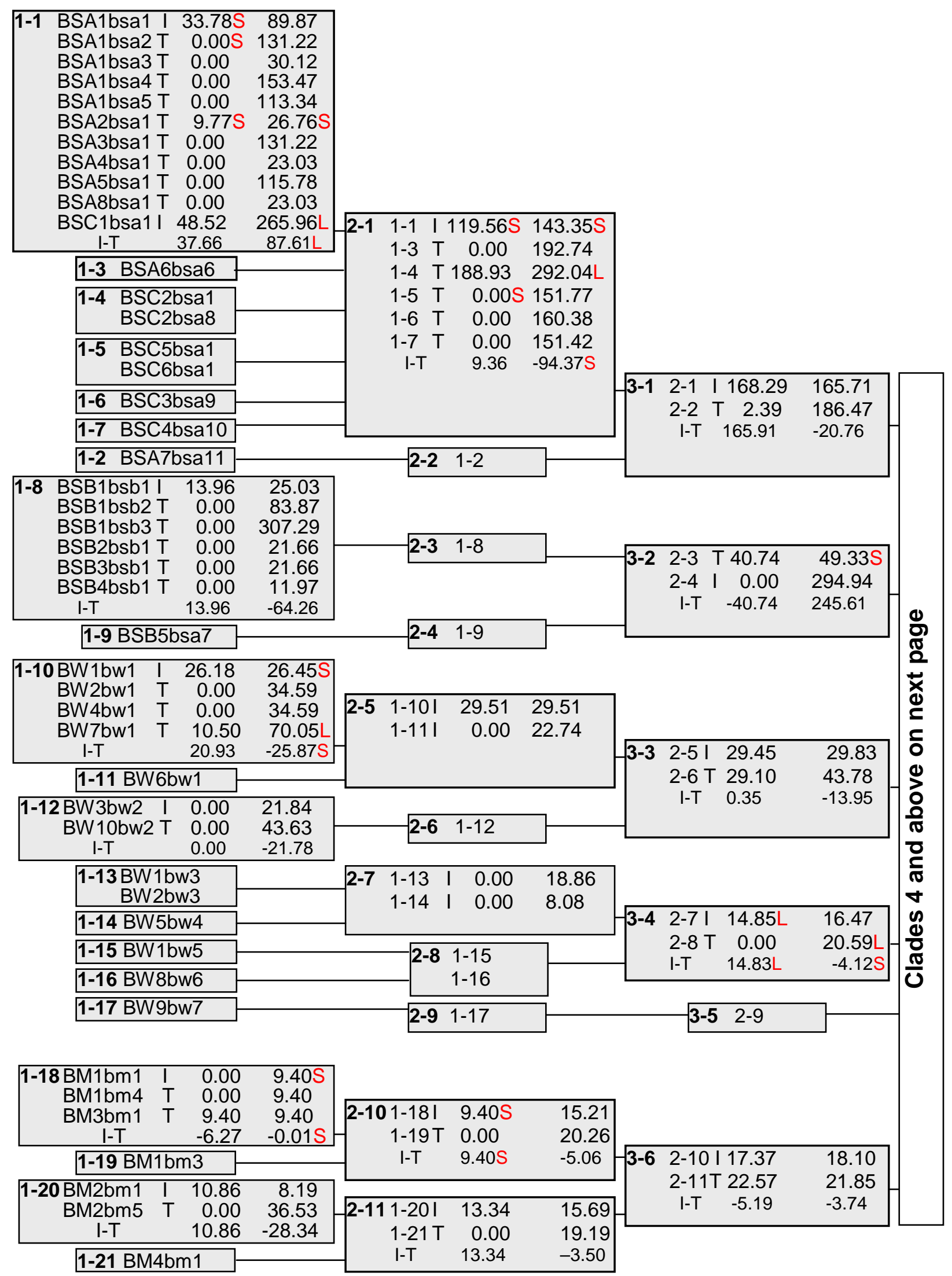



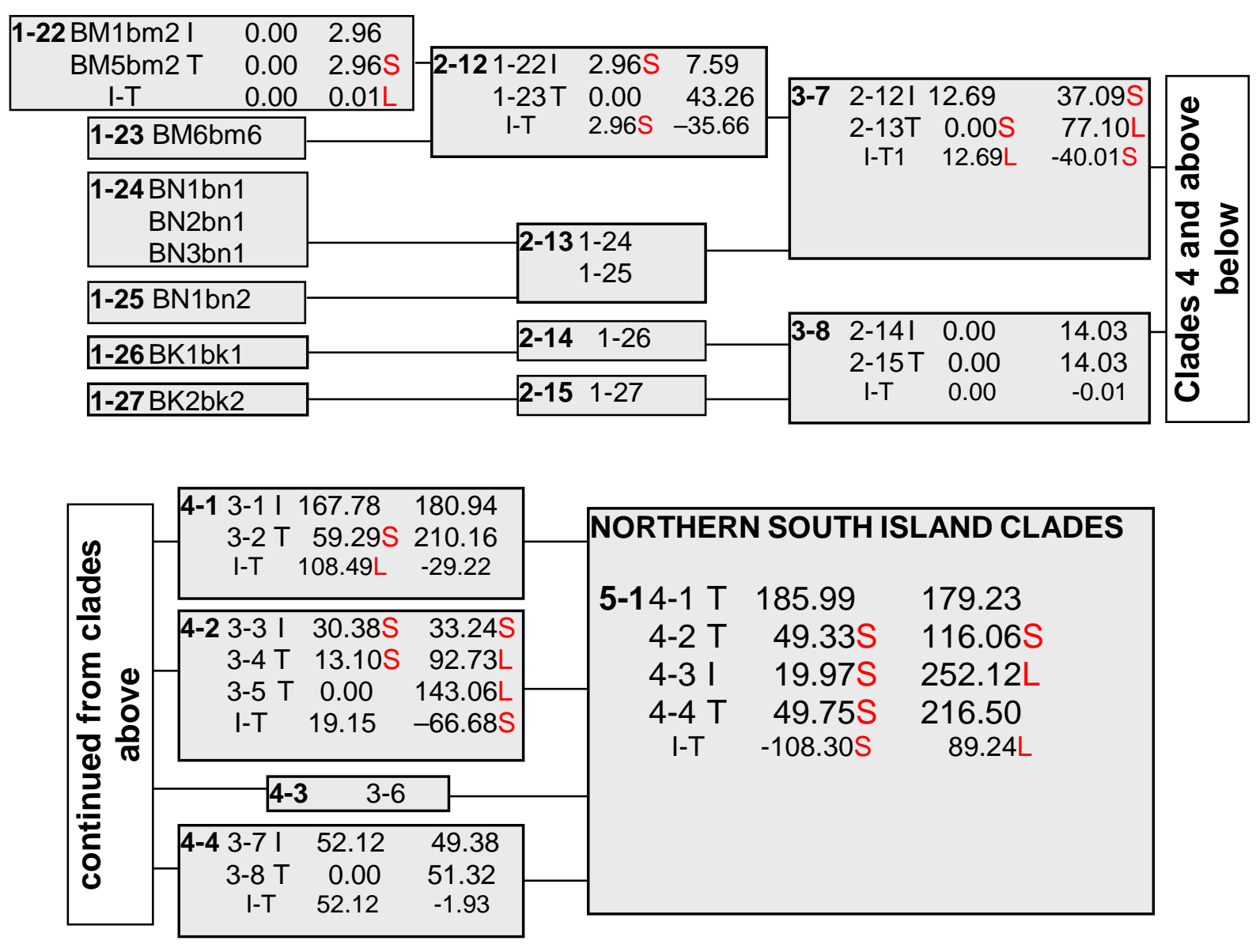

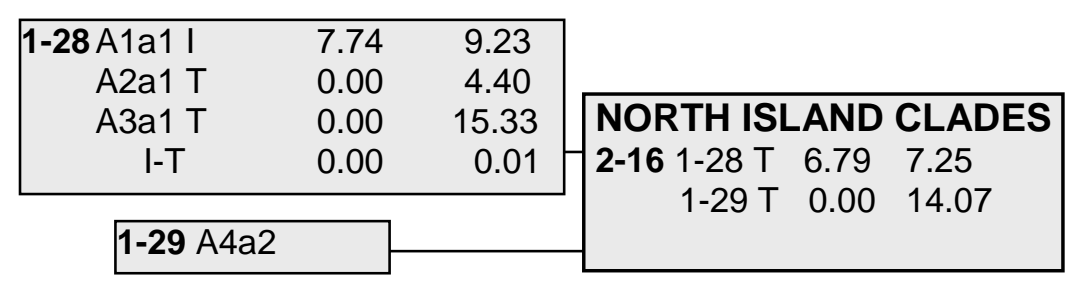

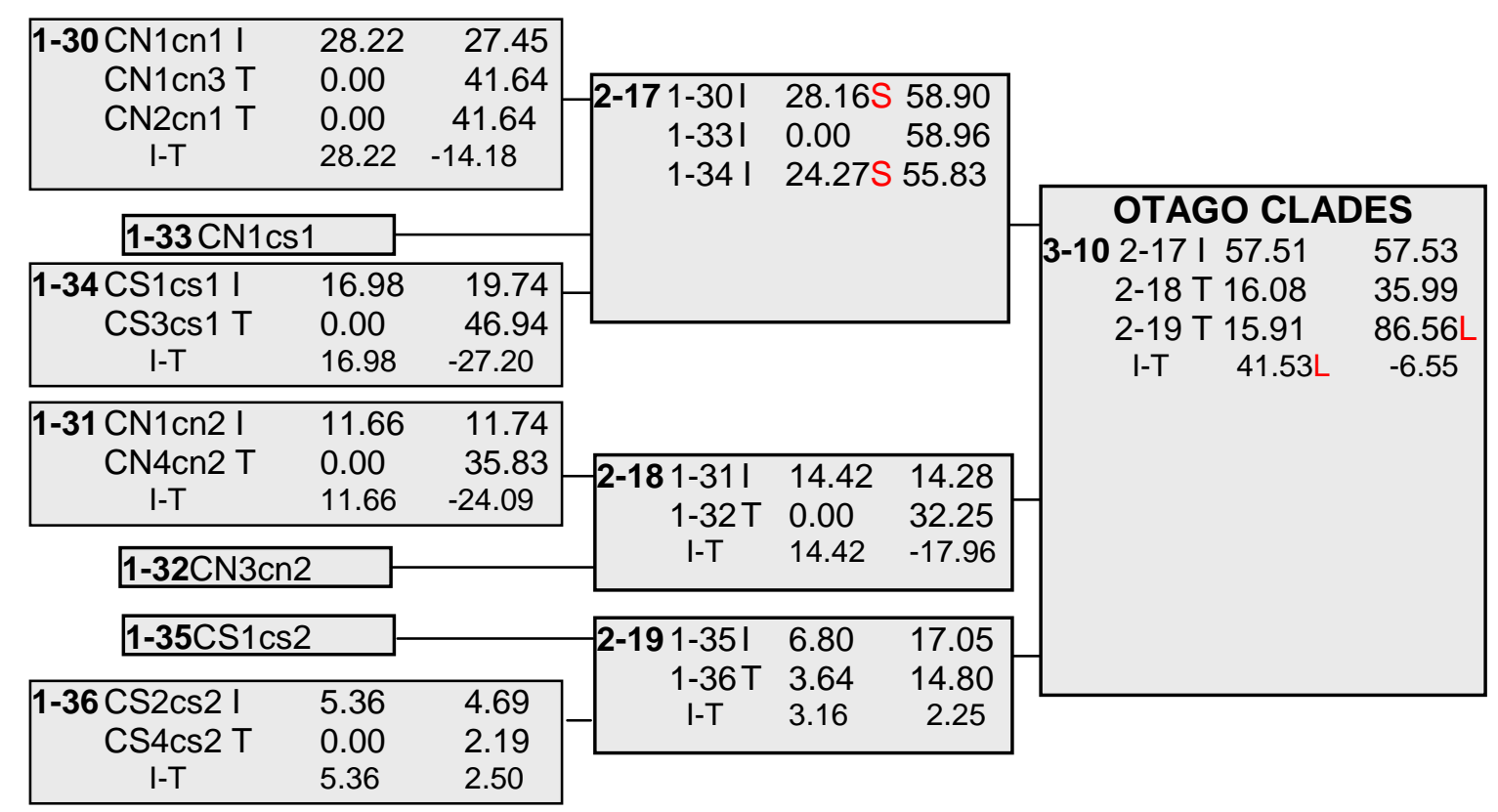


Figure 3.5 Summary of Geodis output results for NCA of 71 Total-mtDNA Maoricicada campbelli haplotypes. Clade names as in Fig 4. As noted elsewhere, the Otago and North Island clades are too divergent to be included in the same nesting sets as the northern South Island clades. See Appendix $F$ for complete raw data. The letter I denotes interior clade, the letter $\mathrm{T}$ denotes tip clade. Numbers following clade names are geographical distances for "within clade", followed by "nested clade". Letters following geographical distances denote statistical significance, $\mathrm{L}=$ large, $\mathrm{S}=$ =small.

Table 3.4 Maoricicada campbelli nested clade geographical associations for COI sequence data estimated using the November 2001 Templeton key and Geodis output data. Clade names as in Fig 3.3.

\begin{tabular}{|c|c|c|c|}
\hline 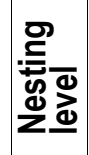 & Clade & Inference key steps & Inference \\
\hline \multirow[t]{7}{*}{1} & $\begin{array}{l}\text { 1-2, 1-8, 1-9, 1-11, } \\
1-12,1-14,1-15,1-18\end{array}$ & 0 & No molecular differentiation \\
\hline & $1-5,1-13$ & 0 & No geographical differentiation \\
\hline & $\begin{array}{l}1-1,1-3,1-7,1-10 \\
1-17,1-19\end{array}$ & 1-No & fail to reject $\mathrm{H}_{0}$ : no geographical structure \\
\hline & 1-4 (part SA-C) & $\begin{array}{l}\text { 1-2-3-4-9-10-No: } M \text {. } \\
\text { campbelli probably present } \\
\text { between the Homer Tunnel } \\
\text { and Boundary Stream. }\end{array}$ & $\begin{array}{l}\text { geographic sampling scheme inadequate } \\
\text { to discriminate between fragmentation } \\
\text { and isolation by distance }\end{array}$ \\
\hline & 1-6 (part Waitaki) & 1-2-11-12-No & contiguous range expansion \\
\hline & 1-16 (North Island) & 1-2-11-17-4-No & $\begin{array}{l}\text { restricted gene flow with isolation by } \\
\text { distance }\end{array}$ \\
\hline & 1-20 (part Otago south) & 1-2-3-4-No & $\begin{array}{l}\text { restricted gene flow with isolation by } \\
\text { distance }\end{array}$ \\
\hline \multirow[t]{5}{*}{2} & $2-2$ & 0 & No molecular differentiation \\
\hline & $2-3,2-6,2-8$ & 1-No & fail to reject $\mathrm{H}_{0}$ : no geographical structure \\
\hline & $\begin{array}{l}\text { 2-1 (SA-A), } \\
\text { 2-4 (Waiaki) }\end{array}$ & $1-2-11-12-\mathrm{No}_{0}$ & contiguous range expansion \\
\hline & $\begin{array}{l}2-5 \text { (Marlborough and } \\
\text { Nelson) }\end{array}$ & $\begin{array}{l}\text { 1-2-3-5-15-16-18-No: } M \text {. } \\
\text { campbelli possibly present } \\
\text { between Cobb Reservior and } \\
\text { Marlborough }\end{array}$ & $\begin{array}{l}\text { geographic sampling scheme inadequate } \\
\text { to discriminate between fragmentation, } \\
\text { range expansion and isolation by } \\
\text { distance (short distance movements). }\end{array}$ \\
\hline & 2-9 (Otago south) & 1-2-3-4-No & $\begin{array}{l}\text { restricted gene flow with isolation by } \\
\text { distance }\end{array}$ \\
\hline \multirow[t]{3}{*}{3} & 3-1 (SA and Waitaki) & 1-2-11-12-13-Yes & long distance colonisation \\
\hline & 3-2 (NMK) & 1-2-11-12-No & contiguous range expansion \\
\hline & 3-4 (Otago) & 1-2-No & inconclusive outcome \\
\hline 4 & 4-1 (northern SI) & 1-2-No & inconclusive outcome \\
\hline
\end{tabular}


Table 3.5 Maoricicada campbelli nested clade geographical associations for TotalmtDNA sequence data estimated using the November 2001 Templeton NCA key and Geodis output data. Clade names as in Fig 4. See Fig 5 for a visual representation of clade geographical ranges.

\begin{tabular}{|c|c|c|c|}
\hline 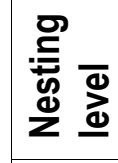 & Clade & Inference key steps & Inference \\
\hline \multirow[t]{6}{*}{1} & $\begin{array}{l}1-2,1-3,1-6,1-7,1-9,1- \\
11,1-14,1-15,1-16,1- \\
17,1-19,1-21,1-25,1- \\
26,1-27\end{array}$ & 0 & No molecular differentiation \\
\hline & $1-5,1-13,1-24$ & 0 & No geographical differentiation \\
\hline & $\begin{array}{l}1-4,1-8,1-12,1-20 \\
1-28,1-30,1-31,1-34,1- \\
36\end{array}$ & 1-No & fail to reject $\mathrm{H}_{0}$ : no geographical structure \\
\hline & $\begin{array}{l}\text { 1-1 (part SA-A and } \\
\text { part SA-C) }\end{array}$ & $\begin{array}{l}\text { 1-2-3-4-9-10-No: campbelli } \\
\text { present but not sampled from } \\
\text { the West Coast (Franz Josef } \\
\text { Lake Paringa etc). }\end{array}$ & $\begin{array}{l}\text { geographic sampling scheme inadequate } \\
\text { to discriminate between fragmentation } \\
\text { and isolation by distance }\end{array}$ \\
\hline & $\begin{array}{l}\text { 1-10 (part Waitaki), } \\
\text { 1-18 (part Marlborough) }\end{array}$ & 1-2-11-17-No & inconclusive outcome \\
\hline & 1-22 (part Marlborough) & $\begin{array}{l}\text { 1-2-11-17-4-9-10-No: } \\
\text { campbelli probably present } \\
\text { between Rag and Famish } \\
\text { Creek and Lake Sedgemere. }\end{array}$ & $\begin{array}{l}\text { geographic sampling scheme inadequate } \\
\text { to discriminate between fragmentation } \\
\text { and isolation by distance }\end{array}$ \\
\hline
\end{tabular}

\begin{tabular}{|c|c|c|c|}
\hline \multirow[t]{6}{*}{2} & $\begin{array}{l}2-2,2-3,2-4,2-6,2-9,2- \\
14,2-15\end{array}$ & 0 & No molecular differentiation \\
\hline & $2-8,2-13$ & 0 & No geographical differentiation \\
\hline & $\begin{array}{l}2-5,2-11,2-16,2-18,2- \\
19\end{array}$ & 1-No & $\begin{array}{l}\text { Fail to reject } \mathrm{H}_{0} \text { : no geographical } \\
\text { structure }\end{array}$ \\
\hline & $\begin{array}{l}\text { 2-1 (part SA-A } \\
\text { and SA-C), } \\
\text { 2-10 (part Marlborough) }\end{array}$ & 1-2-11-12-No & contiguous range expansion \\
\hline & 2-12 (part Marlborough) & 1-2-11-17-No & inconclusive outcome \\
\hline & 2-17 (part Otago) & 1-2-11-17-4-9-No & past fragmentation \\
\hline \multirow[t]{4}{*}{3} & $3-5,3-10$ & 0 & No molecular differentiation \\
\hline & $3-1,3-3,3-6,3-8$ & 1-No & fail to reject $\mathrm{H}_{0}$ : no geographical structure \\
\hline & 3-2 (SA-B) & 1-2-11-17-4-No & $\begin{array}{l}\text { restricted gene flow with isolation by } \\
\text { distance }\end{array}$ \\
\hline & 3-4 (part Waitaki) & $\begin{array}{l}\text { 1-2-3-5-6-7-8-No: campbelli } \\
\text { may be present between Kye } \\
\text { Burn River and Awakino. }\end{array}$ & $\begin{array}{l}\text { geographic sampling scheme inadequate } \\
\text { to discriminate between isolation by } \\
\text { distance (short distance movements) vs } \\
\text { long distance dispersal }\end{array}$ \\
\hline
\end{tabular}


shows the Geodis input and raw Geodis output. An output summary for the Total-mtDNA analysis is shown in Fig 3.5. The Geodis geographical associations were visualised by mapping out the current clade ranges (shown here for the Total-mtDNA dataset only, Fig 3.6). Contiguous range expansion was seen in clades where older haplotypes were found throughout the whole range of the clade, while younger haplotypes were found only at the extremities of an apparently expanding edge. Past fragmentation was seen in only one clade that had nested clades with little overlap in their ranges. Restricted gene flow with isolation by distance was seen in clades with some populations a short distance away with some different but reasonably closely related nested clades. Long distance colonization occurred only in two of the largest clades, with the nested clades occupying large non-overlapping territories.

Many of the clades, especially those at the lower-levels, could not be analysed due to the lack of either molecular or geographic structuring (Tables 3.4, 3.5). The clades that did show significant Geodis results were analysed using the 2001 version of the key from Templeton (1995) in conjunction with the maps of the clade locations (Total-mtDNA: Fig 3.6). The new key (Templeton 2004) was also trialled and yielded no different results. Many lower-level and some higherlevel clades failed to reject the null hypothesis of no geographical associations (Tables 3.4, 3.5). Several clades were entirely composed of interior or tip clades and had inconclusive outcomes (Tables 3.4, 3.5). Historical population movement of two COI and five Total-mtDNA clades was uncertain because of inadequate sampling (Table 3.5). In each of these cases, $M$. campbelli were probably currently present in an area in between clade ranges that would 
otherwise show significant patterning. Such results from earlier analyses on an incomplete dataset were used to determine the most important areas for subsequent field sampling (Templeton 1998, Templeton 2004). The historical population movements for eight $\mathrm{COI}$ and seven Total-mtDNA clades were able to be estimated (Tables 3.4, 3.5). The COI dataset had far fewer clades than the Total-mtDNA, however almost $50 \%$ more sequenced individuals. This increase in power for the $\mathrm{COI}$ dataset is presumably the reason for the reduction in ambiguous clades and the slight increase in positively estimated clades. While following most of the queries in the Templeton (Nov 2001) key led to unambiguous answers, it was at times difficult to interpret the difference between one of the queries that lead to either restricted gene flow with isolation by distance versus past fragmentation (step 4, Templeton Nov 2001 key). While all attempts were made to be impartial, in some cases it was felt that either answer could be correct, as nested clades in most such cases were somewhat overlapping and had medium amounts of genetic difference, and sometimes did not strongly correlate with either scenario.

Total-mtDNA results indicate the northern-SI clade formed through long distance colonisation (Table 3.5), with populations spreading southward from the NMK clade over Island Saddle, a 1365m high drainage-change (Fig 3.6), to populate the Waitaki and Southern Alps areas. Today, the populations in the Marlborough and SA-A clades are completely separated by only ca. $5 \mathrm{~km}$ over Island Saddle, and yet are only distantly related in mtDNA. The COI data indicates that the NMK clade has undergone contiguous range expansion out from the Marlborough populations to the Nelson and Kaikoura populations 
(Table 3.4), and Total-mtDNA data also suggests the southern part of the Marlborough clade has undergone contiguous range expansion (Table 3.5). Most of the SA clades do not have enough data to estimate their geographical history. Some of the clades have significant unsampled areas in between the nested clades that could harbour M. campbelli, making it impossible to distinguish between range expansion, fragmentation and isolation by distance (Tables 3.4, 3.5). Total mtDNA indicates that the SA-B clade has undergone restricted gene flow with isolation by distance (Table 3.5), with one individual approximately $150 \mathrm{~km}$ further north than the rest. This individual is in the same population (NC.ANP, Waimakariri River (Fig 3.1)) as other individuals from the SA-A clade, and in the Total-mtDNA NCA diagram is the haplotype BSB5bsa7 linking SA-B with SA-A (Fig 3.4). Parts of the Waitaki clade have undergone contiguous range expansion from the COI data (Table 3.4). The COI NCA diagram nests Waitaki and the Southern Alps together, and estimates they spread into their current ranges through long distance colonization (Table 3.4).

The North Island clade with the fewest haplotypes and individuals sequenced had mostly non-significant geographical associations. COI data indicated that the most distantly located population (Tukino Ski Field) had undergone restricted gene flow with isolation by distance.

From the Total-mtDNA data, the whole Otago clade had undergone contiguous range expansion, although much of the more northern and central populations underwent range fragmentation at some time. COI data indicated the Otago south clade had undergone restricted gene flow with isolation by distance of the more southern populations. 
The Otago clade contacts the northern South Island clades in three different places, and in three different clades, Wataki, SA-C, and SA-B (Figs 3.1, 3.6). These three contact zones were predicted by Buckley et al. (2001a). At Lake Ohau, the northern end of the Otago clade contacts the southwestern edge of the Waitaki clade. The closest populations not in contact are found $12 \mathrm{~km}$ north (MK.HVI, four individuals sequenced) and $25 \mathrm{~km}$ south (MK.AHU, 10 individuals sequenced) of this contact zone. At Mt Creighton, on the northcentral edge of Lake Wakitipu, the Otago clade contacts the SA-B clade. The closest populations not in contact are $18 \mathrm{~km}$ north (OL.BBN, nine individuals sequenced) and $11 \mathrm{~km}$ south (OL.TWE, eight individuals sequenced) from this contact zone. The single individual found approximately $1.5 \mathrm{~km}$ south at the OL.CRS collection site was from the SA-C clade, rather than the Otago clade as would have been predicted; OL.CRS is probably a population that is also within this contact zone. These two areas of contact are relatively narrow, measuring only a maximum of approximately $31 \mathrm{~km}$ (Ohau) and $37 \mathrm{~km}(\mathrm{Mt}$ Creighton). At these two contact zones, haplotypes from both clades were found at both the lowest and highest elevation sampled in the vicinity (Ohau, 538-1500m and Mt Creighton, 400-535m). At Mararora River, south of Queenstown, the southwestern edge of the Otago clade contacts the southeastern edge of the SA-C clade. The closest populations not in contact are found $22 \mathrm{~km}$ west (FD.BST, one individual sequenced) and $24 \mathrm{~km}$ east (OL.ORE, one individual sequenced) from this contact zone. The contact zone and nearby populations at Mararora river were the least-well sampled of the three, with this contact zone being a maximum of $46 \mathrm{~km}$ wide. Further sampling would be necessary to determine the exact width of any of the contact zones, 
as in all cases there are many kilometres of unsampled suitable habitat between populations in contact and nearby populations representing a single clade. A fourth contact area predicted by Buckley et al. 2001a between the Waitaki and Otago clades in the Omarama area was not found in this study, but would probably be found with further sampling.

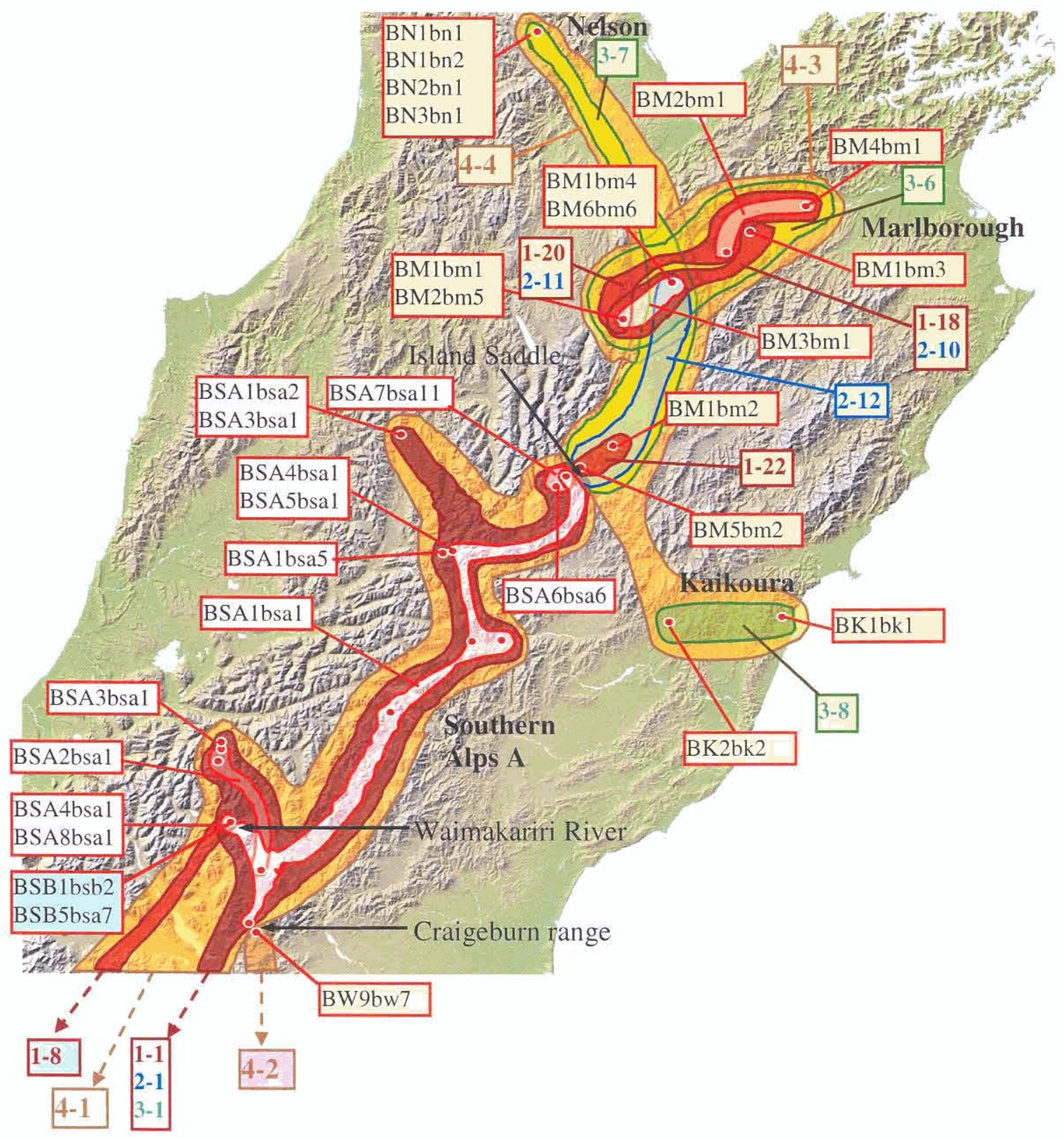




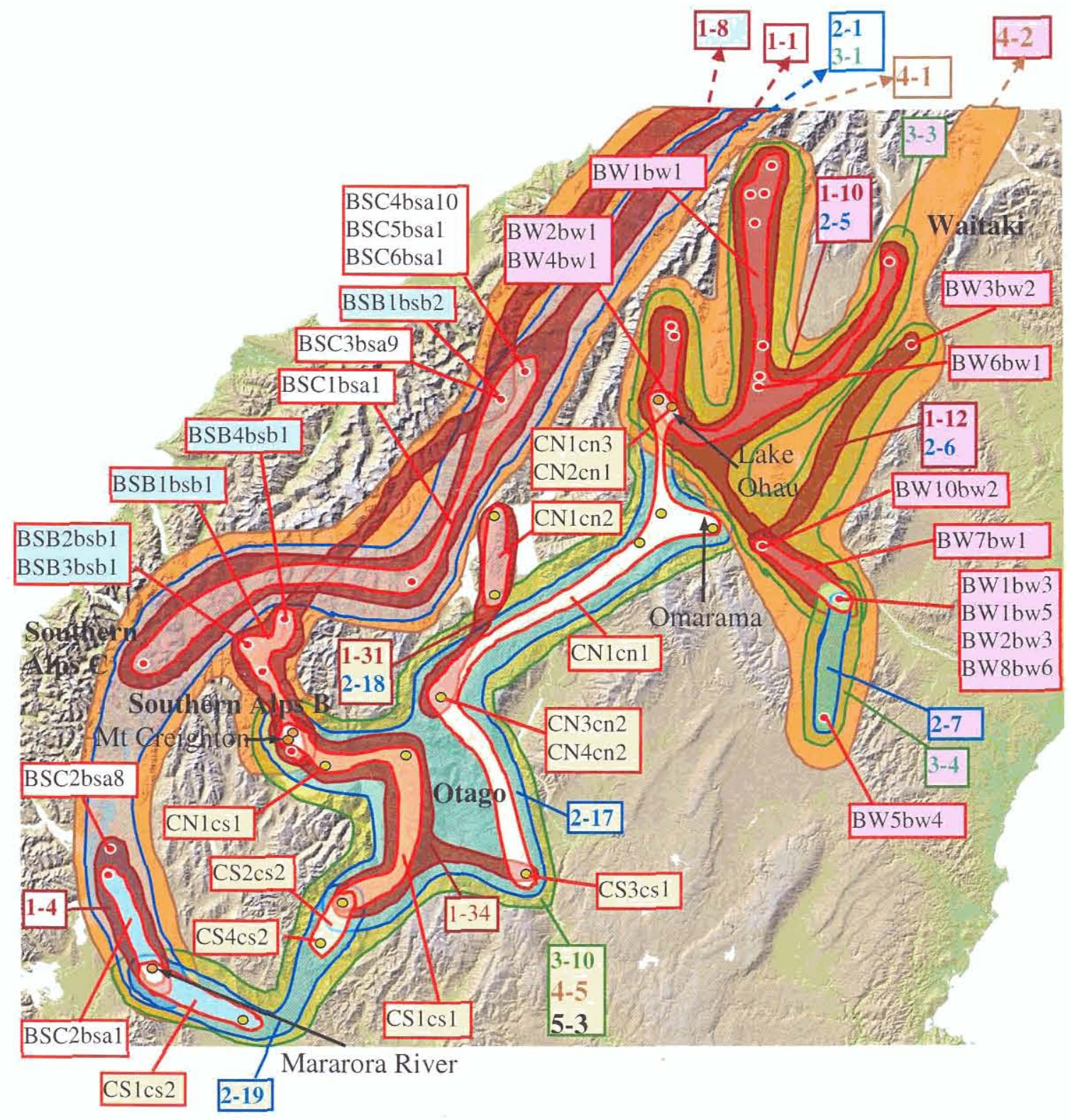

Figure 3.6 Total-mtDNA nested clade geographic locations for South Island populations of Maoricicada campbelli. The location of all haplotypes and many higher clades are shown. Red with white internal are haplotypes, brown $=1$-step clades, blue $=2$-step clades, green $=3$-step clades, orange $=4$-step clades. All localities shown on these two pictures, except for the Otago clades, are joined into the same 5-step clade (5-1, northernSI clade). Within text boxes white $=$ Southern Alps $A$ and $C$, blue $=$ Southern Alps B, pink = Waitaki, yellow = NMK and Otago. Some geographical features mentioned in the text are indicated. DEM courtesy of GeographX, NZ. 


\section{Discussion}

\subsection{Summary of new Maoricicada campbelli findings}

The main patterns resolved by the Maoricicada campbelli phylogenetic and phylogeographical analyses are 1) the main clades, either two (North Island+northern South Island and Otago), or three (North Island, northern-South Island and Otago), are separated by deep genetic divergence and could represent different species; 2) the population structuring suggests a glacial hypothesis for the distribution of M. campbelli throughout the South Island, rather than one mediated by lateral movement along the Alpine Fault; 3) there are three secondary contact zones between the northern South Island and Otago clades, represented by three northern-South Island subclades; and 4) $M$. campbelli in the South Island suggest a conceptual ring species.

\subsection{Maoricicada campbelli phylogeography and historical population movement}

\subsubsection{Overall findings}

This study employed both phylogenetic (ML and Bayesian) and phylogeographical (NCA) methods of testing relationships within a species. NCA is a method employed to statistically explain the present day range of a species via historical associations of populations, and works best within closely related species with restricted dispersal (Templeton et al. 1987). Maoricicada campbelli was believed to be a prime candidate for NCA as it was widespread, and initial data (Buckley et al. 2001a) indicated a high degree of phylogeographic structuring suggesting a low dispersal rate. These NCA 
analyses found three mtDNA divisions within M. campbelli (NI, northern-SI and Otago) (Figs 3.3, 3.4) that were too deep to be analysed together. Thus, NCA can shed no light on the historical associations of these main clades, but does provide support for the $\mathrm{ML}$ tree where $\mathrm{NI}$ is sister to northern-SI, rather than the Bayesian tree that nested NI within the northern-SI clade (Fig 3.2).

Two NCAs were conducted, one on the COI data only, as there were more individuals sequenced for this gene, and one on Total-mtDNA (COI+A6-A8) as there were more haplotypes found which allowed for a finer resolution of geographical associations. Despite the large number of individuals and populations sampled, more data are needed in future to resolve the history of many clades. The COI dataset had almost $50 \%$ more individuals sequenced (Table 3.2), and even though it had fewer clades overall, slightly more clades had positive results and fewer clades had ambiguous results (Tables 3.4, 3.5). Sequencing additional $M$. campbelli individuals from populations already sampled in these analyses will increase the power in the geographical associations and in some cases may provide more information for future geographical association analyses than sequencing new populations represented by few individuals. It is possible that some of the geographical associations shown here are false, however most seem intuitive from a visual inspection of clade ranges (Fig 3.6) and a knowledge of New Zealand palaeohistory. NCA estimates should be checked using a second locus, as NCA does not provide estimates of error and is known to fail under certain scenarios (Templeton 2004). The use of multiple loci would guard against incorrect geographical associations, which are unlikely to occur multiple times 
(Templeton 2004). Even though there are two NCA analyses shown here, mtDNA is inherited as a unit, therefore different mtDNA genes cannot be used to infer different histories. Sequencing of additional loci to reduce the likelihood of multiple errors in geographical associations is planned for a future study.

\subsubsection{Evolution of the North Island clade}

The placement of the $\mathrm{NI}$ clade in the phylogenetic trees is ambiguous, with low support for the placement of the NI clade either outside or within the NMK clade (Fig 3.2). The NI clade is probably more likely to have dispersed north from a Marlborough clade restricted during glacial periods to populations in northwest Nelson, than to have survived in refuges in the North Island and later spread south to populate the northern South Island. Evidence for a glacial refugium for plants in northwest Nelson is strong (Wardle, 1963) while the southern and central North Island territory that $M$. campbelli could have occupied in the glacial times was less hospitable (McGlone 1988). The fact that M. campbelli currently occupies such a restricted range in the North Island, while being found so generally over the South Island supports the hypothesis that $M$. campbelli may not have refuged on the North Island, and may be a recent colonist. However, an alternative explanation could stem from the fact that $M$. campbelli only lives in open habitat, with sparse vegetation, and the North Island is currently more thickly forested than the South Island. M. campbelli may have actually been widespread in parts of the North Island during the glacial periods where there were vast open areas of grass and shrubs (McGlone 1988). With the rapid southward movement of forests after the retreat of the glaciers (McGlone 1988), M. campbelli could have been subsequently retracted to its 
current small range in the open central plateau, similar in method to a retraction in range hypothesised for the alpine scree weta (Trewick et al. 2000). One interesting fact, that there are no Maoricicada spp. on Mt Taranaki/Egmont may provide evidence that this hypothesis is incorrect, as this relatively open glacial maxima habitat stretched to the Mt Taranaki/Egmont area. The Taranaki volcanoes began to form in the early Pleistocene, with Mt Taranaki the last to form beginning approximately 70,000ya (Thornton 1994). Buckley et al. (2001a) dated the $\mathrm{NI}$ clade divergence from the northern-SI clade at 0.9my, which would suggest that the NI clade managed to survive in the North Island for some time. Additionally, the NI clade shows relatively deep divergence which cannot be distinguished from a trifurcation involving it, the NMK clade, and the rest of the northern South Island clade (Figs 3.1, 3.2, 3.4), indicating a probable older colonisation event. In the North Island, territory that $M$. campbelli can occupy may overlap the range of the ambiguously related congeneric species $M$. iolanthe, which appears to have a confused history with M. campbelli (Buckley et al. 2001b). Maoricicada iolanthe is unusual in this genus as it does not sing from rocks or short plants on the ground, but rather inhabits trees, which thus restricts its interactions with the ground-dwelling $M$. campbelli. In M. campbelli and M. iolanthe the songs are almost identical, and if these species did come into contact it is hypothesised that they would attempt to interbreed. A single example of a receptive female $M$. campbelli signalled receptivity to the played-back calls of recorded male $M$. iolanthe, although this female may have been exhibiting increased sexual receptivity seen in older unmated female cicadas (unpublished data). Buckley et al. (2001b) found that M. iolanthe was positioned within the $M$. campbelli lineages in some 
phylogenetic analyses, which could imply mtDNA introgression and indicate hybridisation. Further phylogenetic analyses of all Maoricicada species employing multiple genes (in progress) will provide further information on this pattern.

The nested clade analyses involving geographical associations show little significance within the North Island clades due to the lack of individuals sequenced, and from the small clade ranges. The TO.TSR population on the Tukino Ski Field Rd is further (both genetically and geographically) from the other three populations sampled around Mt Ruapehu, and there is indication of restricted gene flow to that population.

\subsubsection{Evolution of the northern South Island clades}

In the northern-SI clade, populations south of Marlborough were formed by long distance colonisation over Island Saddle. This $1365 \mathrm{~m}$ junction between peaks constitutes a drainage change and does not harbour M. campbelli, even though M. campbelli in other locations (for example Mt Ohau) are found at higher altitudes than this. Maoricicada campbelli present just to the north and south of the saddle are of very different clades, suggesting a single colonisation event over this barrier. Island Saddle also separates two Maoricicada mangu subspecies (Dugdale and Fleming 1978). From the phylogeographical patterning, it appears certain that the southern populations in the northern-SI clade dispersed over Island Saddle prior to the previous interglacial. Dating of these M. campbelli clades using molecular clocks should provide further evidence for this hypothesis. 
All phylogenetic trees support a split between the Marlborough and the Waitaki clade prior to the splitting off of the SA clade (Fig 3.2). The Waitaki clade has the greatest within-clade genetic distance (Table 3.3), and NCA shows that except for the most northern population (MC.SCR), the northern populations of the Waitaki clade are generally older than the southern ones, indicating a spread southward (Fig 3.6). The most northern population is distantly related to the other Waitaki haplotypes (Fig 3.4), and shows a spread northwards (Fig 3.6). This seems to indicate that the Waitaki population refuged over at least the last glacial maximum south of Island Saddle, possibly in the eastern tundra that was present in the central South Island during the glacial times, a similar habitat to areas on the North Island that are also postulated (above) to have been able to harbour $M$. campbelli throughout glacial periods.

The Southern Alps clades also show evidence of refuging south of northwest Nelson in at least the previous interglacial. NCA indicates that this clade formed though restricted gene flow with isolation by distance from the SA-B subclade to the $S A-A+S A-C$ subclade. The phylogenetic trees show that the SA-B subclade is sister to the SA-A+SA-C subclade (Fig 3.2). Geographically, SA-B is split over the central Southern Alps (Fig 3.6), and NCA estimates that this subclade also formed though restricted gene flow with isolation by distance. Part of the SA-A and SA-C subclades are estimated to have been formed through range expansion. It is possible that the SA-B clade split from Marlborough or early northern Waitaki populations and went south along the Southern Alps during a previous interglacial, and then traveled back north to form the progenitor of the SA-A+SA-C populations. The SA-B clade joins to the 
SA-A clade in the Total-mtDNA NCA diagram through a younger haplotype (BSB5bsa7, Fig 3.4) found only in the most northern population (NC.ANP, Fig 3.6). The SA-A clade would then have travelled south to form the closely related SA-C clade. Unsampled populations recorded from Fox and Franz Joseph glaciers may provide evidence for this scenario. The ambiguity seen in the Total-mtDNA diagram where the Marlborough population could be joined to the SA populations through SA-A would indicate a more simple spread southward of the SA-B and SA-C clades from the SA-A clade. Although this scenario is contradicted by the phylogenetic analysis, it can be described as follows: once the SA-A clade was isolated from the Marlborough clade south of Island Saddle, it moved quickly southwards during one of the interglacials down the backbone of the Southern Alps. These populations (SA-B) became isolated from the remaining northern populations (SA-A) during one of the glacial cycles, which did not completely wipe out the SA-B clade refuging in the south. During one of the next interglacials, the SA-A clade again travelled down the backbone of the Southern Alps, and was again wiped out in the central portion to form SA$\mathrm{C}$ clade that also managed to refuge somewhere in the south. Although the likelihood tree has low support for the grouping ((SA-A, SA-C), SA-B), the Bayesian tree shows strong support for this scenario and is preferred because of the increased reality of the partitioned model of evolution (Fig 3.2). The COI NCA cladogram (Fig 3.3) supports linking the NMK clade through the SA-A clade, but has an ambiguity where instead the NMK clade could be joined to the Waitaki clade. Further sequencing of either more individual M. campbelli for the same gene regions to attempt to find new linking haplotypes, or a second gene region will be useful to provide stronger support for our preferred hypothesis. 
From the NCA, populations in the central Marlborough clade have undergone contiguous range expansion, and $\mathrm{COI}$ indicates that the whole NMK clade has undergone contiguous range expansion. This COI NCA result seems unlikely, as the populations in Nelson, Marlborough and Kaikoura are separated by large areas with no sampled $M$. campbelli, and the genetic variation between these clades is high compared to the variation within. Lower-level NMK clades from both $\mathrm{COI}$ and Total-mtDNA data find the geographic sampling scheme inadequate to discriminate between fragmentation, range expansion and isolation by distance (short distance movements), because M. campbelli may be present in the areas between the Central Marlborough clade and both the Nelson and Kaikoura clades.

\subsubsection{Evolution of the Otago clade}

The Otago clade has undergone contiguous range expansion, with restricted gene flow to the most southern populations. Even though this clade should have had almost as much time to spread northwards after the retreat of the ice as the northern-SI clade has had to spread southwards, it has spread only a fraction of the distance. If during the last glacial maximum the SA-B and SA-C clades refuged in areas near the southern Southern Alps, and the Waitaki clade refuged in the tundra in the far east of the central South Island, the restricted distribution of the Otago clade could be explained by the short distance between it and the expanding northern-SI clades. 


\subsection{Nested clade outgroup probabilities and rooting}

Rooting nested clade diagrams is considered extremely difficult, and many people do not attempt to root haplotype cladograms (Castelloe and Templeton 1994), instead relying on rooting suggested by phylogenetic methods. The biggest problem is that outgroups usually cannot be considered as they are genetically too distantly related to be included in an intraspecific haplotype network (Crandall and Templeton 1993, Castelloe and Templeton 1994). Additionally, there is little statistical power to assign outgroup probabilities when dealing with such closely related haplotypes (Castelloe and Templeton 1994). Coalescent methods, however, can be used to assign outgroup probabilities to haplotypes (Crandall and Templeton 1993, Castelloe and Templeton 1994). There are several things to consider for each haplotype, including the number of individuals found with that sequence, the position in the cladogram (interior or tip, and distance from midpoint) and the number of other haplotypes it is connected parsimoniously to (Crandall and Templeton 1993). Some problems with using these rooting methods is that uneven sampling can result in a bias towards the interior haplotypes most heavily sampled, which may not always actually be the oldest haplotypes. Using nested clade diagrams in association with phylogenetic methods helps to solve this problem, as phylogenetic trees should be more consistent in the placement of a root (Heulsenbeck et al. 2002, but see Castelloe and Templeton 1994).

Outgroup probabilities calculated by TCS consistently placed the interior haplotype with the most connections (also the most frequent haplotype in most cases), as the haplotype with the highest probability of being the root of the 
cladogram. This method of cladogram rooting was problematic for all clades as judged by comparison to ML and Bayesian phylogenetic trees. Another way to root nested clade diagrams is to take in to account the number of geographical populations that each haplotype appears in, as an older haplotype should be more generally distributed than a derived one. This geographical rooting method was also found to be unlikely, as it simply corroborated the other coalescent methods. In both the ML and Bayesian phylogenetic trees, the support for exactly which haplotype is sister to the rest of the haplotypes for each main clade is not always strong, however, within the haplotype-rich Otago and northern-SI clades at least, there are many haplotypes that branch off before the one preferred by the coalescent methods as the root. Since the rooting of phylogenetic trees is more believable than that from such coalescent methods, because phylogenetic trees take into account other clades and outgroups, it is therefore far more likely that for each clade a different haplotype that by chance was not well sampled, is the actual root.

\section{$\underline{4.4 \text { New Zealand biogeography: dispersal versus vicariance }}$}

The origins of the flora and fauna of New Zealand is a contentious subject, with on-going debate between proponents of a vicariance method arguing that much of the biota has rafted on New Zealand since the Gondwanan break-up 85mya, and proponents of a dispersal scenario believing that most of the biota has arrived more recently. Most of the origins of the endemic species in the flora and fauna of New Zealand are currently believed to be relatively recent and postdate the Gondwana break-up by many millions of years (e.g., Pole 1994, Winkworth et al. 2002). Pole (1994) argued there was evidence that New 
Zealand was completely submerged during the Oligocene drowning event approximately 30 mya, and therefore that all New Zealand biota must be due to recent dispersal. A few ancient Gondwanan elements such as Kauri (Stöckler et al. 2002) and moa (Cooper and Cooper 1995, Cooper et al. 2001) are, however, believed to have survived the Oligocene drowning event, corroborating geological evidence that New Zealand has never been fully submerged since its break from Australia (e.g., Cooper and Cooper 1995). The ancestors of all the New Zealand cicadas are thought to have arrived after the Oligocene drowning (Buckley et al. 2002, Arensburger et al. 2004).

Debate over the origins of distributions within New Zealand also centers on vicariance versus dispersal. Panbiogeographers such as Craw $(1988,1989)$ and Heads (1998) attempt to explain current distributions of taxa throughout New Zealand by using changes in the landscape over time such as lateral displacement due to the alpine fault. Most other biologists explain current patterns of distribution mostly through dispersal, mediated by the landscape and climatic conditions over time. The most dramatic palaeo-environmental modification in New Zealand has occurred on the South Island, where many distributions and speciation events have been attributed to Southern Alps mountain building since the Pliocene and the Quaternary glacial cycles (e.g., Lockhart et al. 2001, McGlone et al. 2001, Trewick et al. 2000, Trewick and Wallace 2001, Smissen et al. 2003, Chin and Gemmel 2004). With the use of molecular techniques, estimates on the age of species and populations are being employed to answer these questions. 


\subsubsection{Hypotheses on the lack of endemism in the central South Island}

Compared to the northern and southern South Island, the central South Island is an endemic-poor area or biotic gap (Cockayne 1926, Willet 1950, Wardle 1963, Craw 1988) (see area marked on Fig 3.1B). There are two main hypotheses to explain this pattern.

\subsubsection{Glacial hypothesis (dispersal) for the lack of endemism in}

\section{the central South Island}

In the glacial scenario, first proposed by Cockayne (1926) and Willet (1950), glaciers in the Quaternary are hypothesised to have covered much of the narrow central $\mathrm{SI}$, and these combined with mountain building in the same area that produced unstable surfaces and severe glacial outwash resulted in the lack of species seen today. Evidence for severe glaciation in the central Southern Alps during the last (Otira) glaciation ca. 18,000 ya indicates that the snow line was lowered approximately $1000 \mathrm{~m}$, and floristic zones lowered approximately 800m (Wardle 1963, Fleming 1980). Previous glacial phases are thought to have been similar (Fleming 1980). Any fauna in severely glaciated areas such as the central Southern Alps would have died out, and the current biota would have recolonised via dispersal from the north or south (Wardle 1963, Fleming 1980, McGlone et al. 2001, Trewick and Wallace 2001). This pattern can be seen in data from Trewick and Wallace (2001) who examined invertebrate species with ranges that include this gap area. Phylogenetic analysis of mtDNA suggested that several taxa that were found in the central SI were most closely related to an ancestor in the north or south suggesting that they had recently colonized this area. Additionally, many plant species in this gap area appear to be recent colonists (Wardle 1963, McGlone et al. 2001). Some Southern Alps 
plant groups have no taxa in the central Southern Alps, e.g. vegetable sheep (Raoulia sp., Smissen et al. 2003) and alpine Whipcord Hebe (Wagstaff \& Wardle 1999). Fleming (1980) described two Maoricicada species, M. cassiope in the north, and M. clamitans in the south that currently almost meet in the central SI, suggesting that these species recently expanded into this area.

\subsubsection{Alpine Fault hypothesis (vicariance) for the lack of}

\section{endemism in the central South Island}

The Alpine Fault hypothesis as discussed by Craw $(1988,1989)$ and Heads (1998) suggests that lateral movement along the Alpine Fault (see Fig 3.1B) caused species disjunctions and created this gap. Craw (1988: Fig 2) presents an area cladogram, based on the distributions of twelve taxa, that suggests that the northern South Island and southern South Island are most closely related biotically while the central South Island is sister to these. Although it is not stated by Heads or Craw, these sister taxa must be presumed to have refuged somewhere such as the inland tundra or coastal areas of the central South Island during the glacial times. Recent estimates of lateral displacement along the alpine fault have predicted faster movement than previously thought, and there is evidence that the rate of movement has been fastest in the Pliocene/Quaternary (Sutherland 1994). Sutherland (1994) suggests that from the mid-Pliocene $(3.4 \mathrm{my})$ there may have been as much as $100 \mathrm{~km}$ of lateral displacement along the alpine fault. Extrapolating from this and using the fastest predicted rate of lateral movement of $40 \mathrm{~mm}$ per year (Sutherland 1994) for the whole ca. $200 \mathrm{~km}$ of central South Island "gap" territory, this predicts that the minimum time for species to become disjunct across this gap would have been around 5my. Taxa found today further to the north or south of this gap 
(such as the NMK and Otago M. campbelli clades separated by a minimum of ca. $600 \mathrm{~km}$ ) would have taken even longer. From this same rate of movement of $40 \mathrm{~mm}$ per year it would have taken $12 \mathrm{my}$ for the whole $480 \mathrm{~km}$ of lateral displacement along the alpine fault. It is, however, unlikely that the rate of movement has continuously been this fast (Sutherland 1994).

\subsubsection{Testing the hypotheses (dispersal versus vicariance) on the}

\section{lack of endemism in the central South Island}

Clearly, depending on the age of the taxa, either scenario (dispersal vs vicariance) is plausible. For related lineages extant in the north and south of the South Island older than 5my (minimum), lateral movement could certainly have contributed to their current distributions. However recent phylogenetic analyses of organisms found on the South Island indicate that many of the lineages showing this disjunct pattern are more closely related than previously thought and postdate most of the lateral slipping (McGlone et al. 2001, Trewick and Wallace 2001). For example Wagstaff and Wardle (1999) found 10 species of alpine whipcord Hebe that were estimated to have evolved over only ca. 500,000 years. This is a group that grows only on the northern and southern ends of the Southern Alps, even though there is apparently good habitat in the central Southern Alps today (Wagstaff \& Wardle 1999). Trewick and Wallace (2001) used a molecular clock to estimate the time of divergence between invertebrate species in 10 genera, finding splitting events across the central South Island gap to be 2-7my. They argued that these estimates postdated the alpine fault hypothesis. Trewick et al. (2000), in their study of the South Island alpine weta species Deinacrida connectens suggested that even the deep divergence between populations of about 4 my was too recent for this 
widespread species to have been formed via alpine fault disjunction. Chin and Gemmel (2004) found that reasonably closely related populations of Celatoblatta cockroaches were distributed on either side of the Southern Alps, suggesting recent movement after glacial retreat.

\section{Conclusions drawn from phylogeographical patterns of Maoricicada}

campbelli clades overall: Most of the South Island M. campbelli clades were found completely, or almost completely, outside the biotic gap (Fig 3.1). Most populations found inside this gap from clades SA-A, SA-C or Otago were younger and stemmed from recent clade expansions. Almost all the populations inside the gap belonged to the Waitaki and SA-B clades, which spanned the entire biotic gap. The Otago clade and its potentially most close relative the NMK clade were found on either side of the biotic gap, with a minimum distance of ca. $600 \mathrm{~km}$ between them. Even though they are distantly related (ca. 2.3 $\pm 0.5 \mathrm{my}$, Buckley et al. 2001a), and may represent separate species, the split between the Otago clade and populations in the northern-SI (and $\mathrm{NI}$ ) clade is too young to have been influenced much by lateral plate movement along the Alpine Fault. The M. campbelli clades that have moved through the biotic gap and come into contact with the Otago clade (Waitaki and SA clades) are descended from the NMK clade or its ancestor. Thus the spread of $M$. campbelli overall fits a glacial hypothesis, with the lineages in the center of the South Island (SA, Waitaki) being more closely related to one of the outer lineages than these outer lineages (NMK, Otago) are to each other. 


\section{Conclusions drawn from phylogeographical patterns of $M$. campbelli}

clades in the Southern Alps: In addition to the broad history of the $M$. campbelli clades in the South Island, the detailed sampling of $M$. campbelli allows a closer look into the distribution of the populations extant in the Southern Alps. Northern and southern Southern Alps clades (SA-A, SA-C) are more closely related to each other than they are to the central Southern Alps clade (SA-B), especially within the A6-A8 gene where the most common haplotype is found in both SA-A and SA-C. From the phylogenetic trees, Southern Alps B is sister to SA-A+SA-C. This pattern is consistent with the area cladogram shown by Craw (1988: Fig 3.2), however, the estimated time of divergence of less than 1my (Buckley et al. 2001a) between these two lineages is far too low for this pattern to be the result of lateral disjunction. The SA-B clade displays a flat comb-like pattern with short branches in the phylogenetic trees, and has only about half the haplotypes of the other two SA clades for the number of individuals sequenced. These factors indicate the SA-B clade has had less time to evolve during modern times and has undergone rapid geographical spread. The locally widespread haplotypes BSA1bsa1 and BSC1bsa1 are separated across the central SI gap by only one mutation in the $\mathrm{COI}$ gene and by a minimum of ca. $300 \mathrm{~km}$. Populations of $M$. campbelli that are yet to be sequenced have been collected from near the Fox and Franz Joseph Glaciers, and should yield further information on the spread of the Southern Alps clades. 


\section{Overall conclusions from Maoricicada campbelli data on the hypotheses} (dispersal vs vicariance) on the lack of endemism in the central South

Island: For all data partitions, M. campbelli seems to fit the glacial hypothesis. Even the most ancient divisions within M. campbelli appear too young to have been able to be subject to more than $100 \mathrm{~km}$ of lateral movement, yet are currently a minimum of ca. $600 \mathrm{~km}$ apart. Thus $M$. campbelli must have been dispersed widely in the late Pliocene, and the populations in the center subsequently wiped out, presumably by glaciers and glacial outwash that were too unstable to support M. campbelli populations for long. Maoricicada campbelli is a very forgiving species, having a wide altitudinal range from sea level to over $1000 \mathrm{~m}$, and is found on a wide range of substrates including dry farm pasture, rocky mountain scree and rocks next to rivers. They can be heard singing in the summer months on the glacial outwash right next to both the Fox and Franz Joseph Glacier rivers. For a species such as this to have been wiped out completely from what was presumably ancient territory indicates that the land must have been very unstable, and climates very unsettled. Because of the relatively deep divergences within the Waitaki clade, there is evidence this clade was able to exist south of Island saddle for at least one glacial cycle and may have refuged in the central South Island during this time. Smissen et al. 2003 discuss the possibility that a population of cushion plant (Raoulia sp.) was able to refuge in this area over at least the last glacial cycle, and Chin and Gemmel (2004) describe multiple haplotypes in cockroaches found in this central zone that suggest populations may have been able to exist there during glacial maxima. It can also be presumed that every time the glaciers retreated, $M$. campbelli refuging in the north (either on the 
North Island or the NMK area, or later in populations further south) and in the Otago area moved towards each other, and populations that came into contact were not able to disperse into each other's territory. Perhaps $M$. campbelli were not able to disperse as far as their current distribution throughout each interglacial, especially during interglacial periods that were cooler and shorter than the current one. Known interglacials as warm as this one have previously occurred (Fleming 1980), and the Waitaki, SA-B and SA-C clades each seem to show a history that must include refuging quite close to the Otago clade.

Whether this inability to penetrate into other populations is through an inability to breed, or stems from the fact that the genes disperse too slowly and the peripheral contact populations are always wiped out in the glacial cycles is as yet unknown. There is no evidence of previous contact zones between the Otago and northern-SI clades in the phylogeographical patterning.

\subsection{Contact zones}

Endler (1977) emphasised that it is not easy to tell whether a contact zone is secondary or primary; a population may split in two and the daughter populations evolve with a stable primary contact zone remaining between them, or a population may be fragmented into two daughter populations by the formation of a barrier, and these spread back around or over the barrier to form a zone of secondary contact. Figure 4.1 details these two possible scenarios, where either 1) populations dispersed to their current clade ranges, or 2) clades evolved in situ via fragmentation. In the fragmentation scenario, contact zones could be primary or secondary, depending whether small amounts of dispersal at clade peripheries was involved, and in the dispersal scenario contact zones would be secondary. Such zones of secondary contact may also stem from 


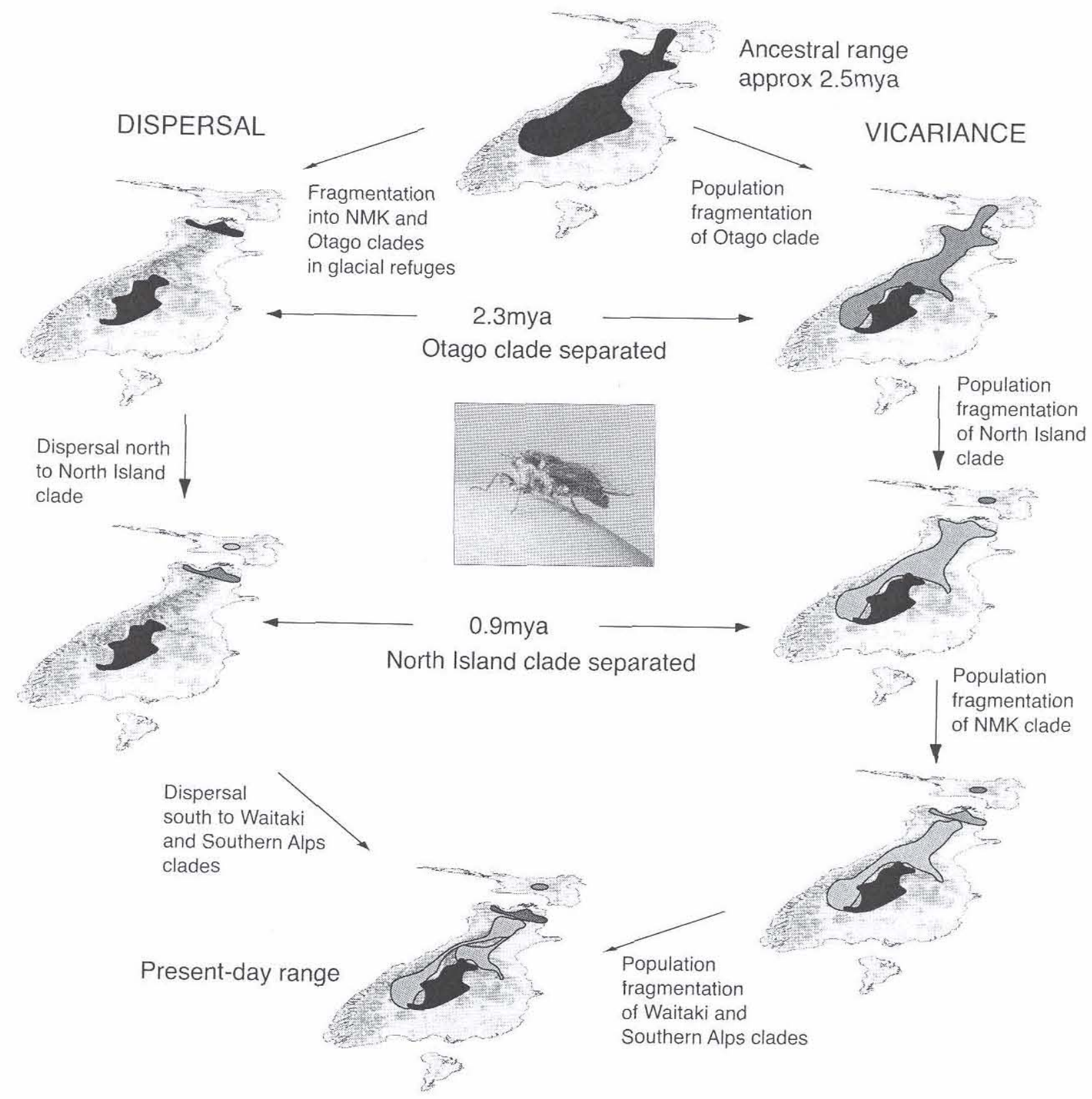

Figure 4.1. Theoretical hypotheses of the distribution of Maoricicada campbelli over ca. 2.3my. Note that the present-day outline of New Zealand is used for all stages without reflecting known landscape changes over this time. Note also that clades are given their present-day range at all times. Ages of Otago and North Island clade separation events estimated by Buckley et al. (2001a). A dispersal scenario predicts that widespread M. campbelli were isolated in two refugia during the first glacial cycle, around 2.3mya. Note that the glacial refugium in the NMK area could have been in the North Island. The isolated Otago clade then remained static. The NMK clade dispersed to the North Island (or vice versa) approximately 0.9 mya, then the NMK clade dispersed southward into the Waitaki and Southern Alps areas, coming into secondary contact with the Otago clade at the very end of this dispersal. A vicariance scenario predicts that a widespread M. campbelli species was subsequently fragmented into isolated populations evolving in situ. The Otago clade was the first to split off ca. 2.3mya, followed by the North Island clade ca. 0.9mya. The NMK clade split off, then lastly the Waitaki and Southern Alps clades separated. In the vicariance hypothesis, which requires the creation of barriers between populations, there would have been small scale dispersal around the edges to create secondary contact zones. Alternatively, population fragmentation could be postulated to follow a parapatric fragmentation model where a strong ecological gradient is coupled with assortative mating related to that gradient. In that case, contact zones would be primary. Inset: M. campbelli male. 
ring-species where a population does not split, but two expanding edges encircle a barrier and come back into contact (Ridley 1993). Newer phylogenetic and phylogeographic methods (such as NCA), include reconstructions of ancestral areas or geographical data, respectively, to give estimates of types of population movement and we can now predict with more certainty whether we are dealing with primary or secondary contact (Templeton 2001).

Areas where geographically isolated lineages come into secondary contact are common in North America and Europe and are often associated with events surrounding the most recent Quaternary glaciation (Hewitt 1996). Contact zones are less obvious well away from significant geological barriers but have been discovered using genetic techniques (Avise et al. 1987). Most contact zones occur between sister species or subspecies, but in several unusual cases more distantly related lineages come into contact, for example, in Herring Gulls (Ridley 1993), in the two 13-year species of the Periodical Cicada Magicicada-decim complex (Simon et al. 2000, Marshall and Cooley 2000, Cooley et al. 2001) and in the now classic ring species of Ensatina salamanders (Jackman and Wake 1994, Moritz et al. 1992, Wake 1997).

Contact zones are important in studying speciation. Examining mating interactions and searching for hybrid individuals in contact zones can suggest whether populations have evolved sufficient differences to have demonstrable barriers to interbreeding (Ridley 1993). Lineages that meet in contact zones may show reproductive character displacement stimulated by selection associated with postmating barriers to reproduction (Rice and Hostert 1993) and sexual signals that help avoid mistakes in mating (Higgie et al. 2000, 
Marshall and Cooley 2000, Servedio 2001). Reproductive character displacement occurs when the individuals in contact have evolved strong premating interbreeding barriers but this change has not yet spread into the rest of the population (Waage 1997, Higgie et al. 2000, Marshall and Cooley 2000). Alternatively, populations in contact may be interbreeding freely and intermediate forms may be found in the contact areas that are then known as hybrid zones.

In the case of $M$. campbelli we have no answer as yet to whether or not the northern-SI and Otago clades are hybridising at the zones of contact. Unpublished data on song differences between these two clades did not indicate character displacement, and combined with mtDNA evidence may indicate hybridisation. Unpublished data on colour characteristics so far only indicates that individuals seem to be generally more alike within populations than between. The fact that the hybrid zones are narrow, however, does suggest a barrier to gene flow and therefore a lack of (successful) interbreeding between the northern-SI and Otago clades. Arnold (1997) suggested that some hybrid zones are narrow because hybrids are more fit in an intermediate habitat. However, we recognised no difference between the habitat in the contact zones and that just outside them. The narrowness of the contact zones could be due to recency of contact but it seems unlikely that all three contact zones are equally recent.

From the phylogenetic trees (Fig 3.2) we cannot tell whether the M. campbelli clades evolved via dispersal or in situ from an initial widespread ancestral population (Fig 4.1), which would not allow us to distinguish between primary or 
secondary contact at the three contact zones. However, NCA does allow predictions of population history and indicates secondary contact because range expansion is indicated for many clades and long distance dispersal (rather than fragmentation) is indicated for the spreading of populations south of Island Saddle that come onto contact with the Otago clade. The only clade demonstrating past fragmentation from the NCA geographical associations is part of the north-central part of the Otago clade.

\subsection{Ring Species}

"Ring species" or "circular overlaps", first proposed by Mayr (1963), are originally described from populations that form a more-or-less circular pattern, where populations disperse both ways around a barrier until they meet on the other side, having accumulated sufficient genetic changes along the way to be reproductively isolated. For example, in the North American Ensatina salamander complex (Jackman and Wake 1994, Moritz et al. 1992, Wake 1997), mountain populations that originated in the north moved south on either side of a central valley experiencing some disjunctions and reconnections over time; the southern-most populations of Ensatina that now come into secondary contact are reproductively isolated. Although there is controversy over whether the Ensatina clades should be called subspecies or species (Highton 1998), this does not detract from the important evolutionary phenomenon they illustrate. Ring species probably provide the best examples for studies in speciation since they display geographically, instead of over thousands or millions of years, how one species may change into another through isolation by distance (Irwin et al. 2001). Ring species demonstrate that small changes over time can lead to the formation of new, reproductively isolated lineages, and that such reproductively 
isolated lineages can form even with significant amounts of gene flow around the chain (Irwin et al. 2001, Irwin and Irwin 2002). Ring species have been proposed in a number of species complexes, however such classifications are constantly being debated, with alternative views classing them as "meta" or "super" -species or simply groups of species that were not recognised (Martens 1996, Highton 1998, Kvist et al. 2003, Liebers 2004). Alternatively, Irwin and Irwin (2002) state that ring species may be more common than currently believed, as some groups that are divided into several species may, in fact, be a single ring species.

The most ideal examples of ring-species are those that are actually physically distributed in a ring, where the contacting reproductively isolated extremes are joined with a circular cline of continuously intergrading populations (Irwin and Irwin 2002). However, a species could be said to be the conceptual equivalent of a ring species if it was formerly widespread and then the central portion was eliminated, isolating the extremes of the range. Later if the central area became habitable again, these extreme populations could expand back into their old locations; if they met in secondary contact zones and were reproductively isolated they would show many of the characteristics of a classic ring species, although some of the linking population chain would have been lost. As most, if not all, examples of ring species are missing some of the intermediate population chain (Irwin and Irwin 2002), conceptual ring species are not as different from the classic circular ring species as one might initially believe. If the linking populations are all missing, we have two unique species. If some of the linking populations are present, then it is possible that the complex may be a ring species. 
Maoricicada campbelli is one such example of evolution in a ring-like form, where a widespread ancestor was later isolated into two populations at the extremes of the range when the central area became uninhabitable. These isolated populations then spread inward during the warmer interglacials, and have now met in secondary contact zones and appear to have at least restricted gene flow (Figs 3.1, 3.6, 4.1). A chain of populations in the northernSI clade spreading from the NMK clade to the lower tips of the SA and Waitaki clades is currently present, as is a small chain of expanded populations within the Otago clade. Although no mating experiments have been conducted, it is probable from the relatively low amount of genetic diversity that successful interbreeding can occur among individuals from all populations within each of the three main clades ( $\mathrm{NI}$, northern-SI and Otago). It is unknown, however, whether successful interbreeding could occur between individuals belonging to these clades. In the ring species scenario, the genetically closest populations linking the northern-SI and Otago clades would be from the central Otago area and the central Marlborough (or North Island) area. It is likely, however, that if individuals from different clades within the contact zones are unable to breed, even these somewhat more closely related populations may have barriers to breeding. The genetically closest haplotypes between the Otago clade and the northern-SI clade are separated by 0.038 uncorrected, 0.047 corrected expected number of substitutions per site, high for within-species genetic divergence (Buckley et al. 2001a), and much higher than the divergence within any one of the main clades (Table 3.3, Appendix 3.3). If contiguous populations outside the contact zones are able to interbreed, and if the individuals in the contact zones are not, then we will have a good argument for describing $M$. 
campbelli as a ring species. Additionally, further analysis of acoustical and morphological data could indicate changes along the chain of linking populations and a ring species-like complex. If populations in the Marlborough area show any evidence of the slight difference in song structure that the Otago clade demonstrates (unpublished data), evidence would be provided for the ring species scenario.

\subsection{Potential speciation and song divergence in Maoricicada campbelli}

Buckley et al. (2001a) described many examples of variation in insect mtDNA and concluded that populations of Maoricicada campbelli were highly divergent for a single species, with corrected mtDNA divergence between some individuals of over 0.05 expected number of substitutions per site. These analyses confirm this (Table 3.2, Appendix 3.3), and support the hypothesis that the NI, Otago and northern-SI clades, or North Island+northern-SI and Otago could represent cryptic species. Each of these clades inhabits a unique geographical range, except where the northern-SI clade and the Otago clade contact in narrow zones (Figs 3.1, 3.6). Other studies of New Zealand alpine or subalpine taxa have also demonstrated higher-than-usual levels of molecular divergence, sometimes between populations that otherwise show no discernable differences. For example Trewick et al. (2000) found that discretely distributed populations of an alpine scree weta, Deinacrida connectens, had up to 0.076 uncorrected, 0.130 corrected $(G T R+l+\Gamma)$ expected number of substitutions per site. Trewick et al. (2000) found no other information from nuclear markers that corroborated these findings. 
The songs of cicadas facilitate contact between the sexes, and represent the primary isolating mechanism between different species. Cryptic species of cicadas are often discovered first by recognizing a unique call (e.g., Marshall and Cooley 2000, Gogala and Trilar 2004). The M. campbelli in the Otago clade have a subtle difference in their song that lends further evidence to the cryptic species hypothesis (unpublished data). However, there is also a suggestion of very small differences between some populations within the northern-SI clades, and so far no song differences have been seen in the North Island (unpublished data). The eastern North American cicada Magicicada neotredecim exhibits character displacement in the form of song pitch elevation where it contacts the closely related species Magicicada tredecim (Marshall and Cooley 2000). This has been shown to isolate these two species in the contact zone, whereas individuals from outside the contact zone will mate freely (Marshall and Cooley 2000, Simon et al. 2000, Cooley et al. 2001). No character displacement has been found in preliminary analyses of the songs of M. campbelli in the contact zones, however more intensive song analysis will need to be conducted before this is concluded. Individuals from the zones of contact were not known to represent two different clades at the time of collection. Preliminary analyses of individual songs from these populations indicate the song difference may be obscure in such zones, which could indicate hybridization is occurring. Since the Maoricicada species exhibit mostly temporal differences in song, rather than pitch differences, it is possible that a more subtle song difference is enough to isolate species, possibly by relying on differences in timed female response. Further work on song structure and mate-choice will be done to determine if the song difference 
observed between the northern-SI and Otago clades is isolating or not. One example of a receptive female $M$. campbelli (the same individual that also responded to $M$. iolanthe calls) responded to play-back calls of males of all three main M. campbelli clades (unpublished data). The fact that there is only a subtle difference between the songs of these different $M$. campbelli clades (and also that of the ambiguously related $M$. iolanthe) may indicate a difference in cicadas isolated by temporal rather than pitch-mediated song differences. The common ancestor of Magicicada tredecim and M. neotredecim $+M$.

septendecim is estimated to be about a million years old (Simon et al. 2000) and $M$. tredecim and M. neotredecim show marked character displacement in song when in contact (Marshall and Cooley 2000). The Maoricicada campbelli clades presently in contact diverged ca. 2.3mya (Buckley et al. 2001a), yet show no evidence of character displacement. Several possible scenarios exist: 1) there is no interbreeding because the small temporal song difference discovered is enough to isolate the clades, 2) the clades are interbreeding freely at the contact zones with no negative effects from hybridisation or 3) the clades are not successfully interbreeding but came into contact too recently for additional song divergence to have occurred. Mate-choice experiments will lend support to one of these hypotheses, and also help to determine the specific status of the main clades. If there was unsuccessful interbreeding between any of the clades, such mating studies could also help break up the trifurcation between the $\mathrm{NMK}, \mathrm{NI}$ and rest of northern-SI clade, and determine whether two ( $\mathrm{NI}+$ northern-SI, Otago), three ( $\mathrm{NI}$, northern-SI, Otago) or four ( $\mathrm{NI}$, NMK, Waitaki+SA, Otago) main clades are present. 


\section{Conclusions}

\subsection{Phylogenetic and phylogeographic population structuring conclusions}

The five clades within Maoricicada campbelli found by Buckley et al. (2001a) were confirmed in this study using both phylogenetic methods and nested clade analysis, with additional resolution into subclades provided by increased population and individual sampling. The placement of these clades into larger clades was problematic, with either two ( $\mathrm{NI}+$ northern-SI, Otago), three ( $\mathrm{NI}$, northern-SI, Otago) or possibly four (NI, NMK, Waitaki+SA, Otago) main clades suggested by different methods of analyses. The population structuring within M. campbelli was detailed enough for NCA to be employed, and for estimates of historical movements to be determined for some clades. NCA estimated that the Otago clade has undergone contiguous range expansion, with fragmentation and restricted gene flow to the most southern populations. In the northern-SI clade, populations south of Marlborough were formed by long distance colonisation over the barrier of Island Saddle. Much of the Waitaki, SA-A and NMK clades were formed by range expansion, while some populations within the Waitaki and SA clades were formed through restricted gene flow with isolation by distance.

\subsection{Population movements: dispersal versus vicariance conclusions}

Almost all $M$. campbelli populations are too recent to have been influenced by vicariance due to Alpine Fault movement, and population structuring of $M$. campbelli within the northern-SI clade is best explained using the glacial theory, that during each Quaternary glacial cycle the center of the South Island is mostly uninhabitable due to heavy glaciation and unstable glacial outwash. The 
SA clade is divided into three subclades with mostly non-overlapping ranges, SA-A in the north, SA-B in the center and SA-C in the South, with the phylogenetic relationship (SA-A, SA-C), SA-B. Clades SA-A and SA-C may have occupied their current distributions longer than clade B, which only recently (re)colonised the central Southern Alps. The range of $M$. campbelli during the more recent glaciations, however, was probably not totally restricted to the northwest Nelson and Otago areas as it may have been during the initial glacial maxima. There is evidence from NCA and molecular dating that $M$. campbelli existed on parts of the North Island, eastern-central South Island within the biotic gap, and in two areas near the southern Southern Alps during later glacial maxima.

\section{$\underline{5.3 \text { Contact zones and ring species conclusions }}$}

Three of the four secondary contact zones predicted in Buckley et al. (2001a) between the northern-SI and Otago clades were found, and the fourth would likely be found with further sampling. These three contact zones were narrow, and each was represented by a different subclade of the northern-SI clade. The populations in contact represent the younger expanding edge of the northern-SI and Otago clades, populations that are separated by approximately 2.3my (Buckley et al. 2001a). As these contact zones represent the young, expanding edges of both clades, M. campbelli has a ring-species-like distribution, missing only part of the chain of populations that were wiped out during previous glacial maxima. The narrowness of the contact zones suggests a barrier to geneflow, and that isolation by distance has led to genetic differentiation. Further study is needed to determine whether the different $M$. 
campbelli clades are reproductively isolated or hybridising at these areas of contact, and therefore whether they represent different species or one, deeply divergent, species. These studies, as well as additional sampling of $M$. campbelli populations, are currently being undertaken.

\section{REFERENCES}

Akaike H. 1973. Information theory and an extension of the maximum likelihood principle. Pp 267-281 In: Second International Symposium on Information Theory, Budapest: Akademiai Kiado.

Akaike H. 1974. A new look at the statistical model identification. IEEE Trans. Aut. Control 19:716-723.

Arnold ML. 1997. Natural hybridisation and evolution. Oxford University Press, Oxford, U.K. 215pp.

Avise JC, Arnold JA, Ball RM, Bermingham E, Lamb T, Neigel JE, Reeb CA, Saunders NC. 1987. Instraspecific phylogeography: The mitochondrial DNA bridge between population genetics and systematics. Annual Review of Ecology and Systematics 18: 489-522.

Batt GE, Braun J, Kohn BP, McDougall I. 2000. Thermochronological analysis of the dynamics of the Southern Alps, New Zealand. Geological Society of America, Bulletin 112: 250-266. 
Buckley TR, Arensburger P, Simon C, Chambers GK. 2002. Combined data, Bayesian phylogenetics, and the origin of the New Zealand cicada genera. Systematic Biology 51: 4-18.

Buckley TR, Simon C, Chambers GK. 2001a. Phylogeography of the New Zealand cicada Maoricicada campbelli based on mitochondrial DNA sequences: ancient clades associated with Cenozoic environmental change. Evolution 55: 1395-1407.

Buckley TR, Simon C, Shimodaira H, Chambers GK. 2001b. Evaluating hypotheses on the origin and evolution of the New Zealand alpine cicadas (Maoricicada) using multiple comparisons of tree topology. Molecular Biology and Evolution 18: 223-234.

Castelloe J, Templeton AR. 1994. Root probabilities for intraspecific gene trees under neutral coalescent theory. Molecular Phylogenetics and Evolution 3: 102-113.

Chamberlain CP, Poage MA. 2000. Reconstructing the paleotopography of mountain belts from the isotopic composition of authigenic minerals. Geology 28: 115-118.

Chin WG, Gemmel, NJ. 2004. Adaptive radiation within New Zealand endemic species of the cockroach genus Celatoblatta Johns (Blattidae): a response to Plio-Pleistocene mountain building and climate change. Molecular Ecology 13: 1507-1518. 
Clement M, Posada D, Crandall KA. 2000. TCS: a computer program to estimate gene genealogies. Molecular Ecology 9: 1657-1659.

Cockayne L. 1926. Monograph on the New Zealand beech forests. Part 1. The ecology of the forests and the taxonomy of the beeches. New Zealand Forest Service Bulletin 4. $71 \mathrm{pp}$.

Cooley JR, Simon C, Marshall DC, Slon K, Ehrhardt C. 2001. Allochronic speciation and reproductive character displacement in periodical cicadas supported by mitochondrial DNA, song pitch, and abdominal sternite coloration data. Molecular Ecology 10: 661-672.

Cooper A, Cooper RA. 1995. The Oligocene bottleneck and New Zealand biota: genetic record of a past environmental crises. Proceedings of the Royal Society of London: Series B, Biological Sciences 261: 293-302.

Cooper A, Lalueza-Fox C, Anderson S, Rambaut A, Austin J, Ward R. 2001. Complete mitochondrial genome sequences of two extinct moas clarify ratite evolution. Nature 709: 704-707.

Cooper RA, Millener PR. 1993. The New Zealand Biota: Historical Background and New Research. Trends in Ecology and Evolution 8: 429-433.

Crandall KA. 1996. Multiple interspecies transmissions of human and simian T-cell leukemia/lymphoma virus type I sequences. Molecular Biology and Evolution 13: 115-131. 
Crandall, KA, Templeton AR. 1993. Empirical tests of some predictions from coalescent theory with applications to intraspecific phylogeny reconstruction. Genetics 134: 959-969.

Craw R. 1988. Continuing the synthesis between panbiogeography, phylogenetic systematics and geology as illustrated by empirical studies on the biogeography of New Zealand and the Chatham Islands. Systematic Zoology 37: 291-310.

Craw R. 1989. New Zealand biogeography: a panbiogeographic approach. New Zealand Journal of Zoology 16: 527-547.

Crosby TK, Dugdale JS, Watt JC. 1998. Area codes for recording specimen localities in the New Zealand subregion. New Zealand Journal of Zoology 25: 175-183.

Cruzan MB, Templeton AR. 2000. Paleoecology and coalescence: Phylogeographic analysis of hypotheses from the fossil record. Trends in Ecology and Evolution 15: 491-496.

Cumber RA. 1952. Notes on the biology of Melampsalta cruentata Fabricius (Hemiptera-Homoptera: Cicadidae), with special references to the nymphal stages. Transactions of the Royal Entomological Society of London 103: 219-237. [Note: Melampsalta cruentata is used here in error for Kikihia muta.] 
Dugdale JS, Fleming CA. 1978. New Zealand cicadas of the genus Maoricicada (Homoptera: Tibicinidae). New Zealand Journal of Zoology 5: 295-340.

Endler JA. 1977. Geographical variation, speciation, and clines. Princeton University Press, Princeton, N.J.

Felsenstein J. 1981. Evolutionary trees from DNA sequences: a maximum likelihood approach. Journal of Molecular Evolution 17: 368-376.

Fleming, CA. 1971. A new species of cicada from rock fans in southern Wellington, with a review of three species with similar songs and habitat. New Zealand Journal of Science 14: 443-479.

Fleming CA. 1980. The Geological History of New Zealand and its Life. Auckland University Press. 141pp.

Fleming CA, Scott GH. 1970. Size differences in cicadas from different plant communities. New Zealand Entomologist 4: 38-42.

Gogala M, Trilar T. 2004. Bioacoustic investigations and taxonomic considerations on the Cicadetta montana species complex (Homoptera: Cicadoidea: Tibicinidae). Anais da Academia Brasileira de Ciências 76: $1-9$. 
Heads M. 1998. Biogeographic disjunction along the Alpine Fault, New Zealand. Biological Journal of the Linnaean Society 63: 161-176.

Hewitt GM. 1996. Some genetic consequences of ice ages, and their role in divergence and speciation. Biological Journal of the Linnaean Society 58: $247-276$.

Higgie M, Chenoweth S, Blows MW. 2000. Natural selection and the reinforcement of mate recognition. Science 290: 462-463.

Hill KBR, Marshall D, Cooley J, Simon C. Crossing Cook Strait: possible human transportation and establishment of two North Island cicadas to the South Island (Kikihia scutellaris (Walker) and K. ochrina (Walker), Hemiptera: Auchenorrhyncha: Cicadidae). In prep for New Zealand Entomologist.

Huelsenbeck JP, Bollback JP, Levine AM. 2002. Inferring the root of a phylogenetic tree. Systematic Biology 51: 32-43.

Huelsenbeck JP, Ronquist F. 2001. MrBayes: Bayesian inference of phylogenetic trees. Bioinformatics 17: 754-755.

Irwin DE, Bensch S, Price TD. 2001. Speciation in a ring. Nature 409: 333337. 
Irwin DE, Irwin JH. 2002. Circular overlaps: Rare demonstrations of speciation. The Auk 119: 596-602.

Jackman TR, Wake DB. 1994. Evolutionary and historical analysis of protein variation in the blotched forms of salamanders of the Ensatina complex (Amphibia: Plethodontidae). Evolution 48: 876-897.

Kvist L, Martens J, Higuchi H, Nazarenko AA, Valchuk OP, Orell M. 2003. Evolution and genetic structure of the great tit (Parus major) complex. Proceedings of the Royal Society of London Series B, Biological Sciences 270: 1447-1454.

Lemmon AR, Moriarty EC. 2004. The importance of proper model assumption in Bayesian phylogenetics. Systematic Biology 53: 265-277.

Liebers D, de Knijff $P$, Helbig AJ. 2004. The herring gull complex is not a ring species. Proceedings of the Royal Society of London Series B, Biological Sciences 271: 893-901.

Lloyd BD. 2003. The demographic history of the New Zealand short-tailed bat Mystacina tuberculata inferred from modified control region sequences. Molecular Ecology 12: 1895-1911.

Marshall DC, Cooley JR. 2000. Reproductive character displacement and speciation in periodical cicadas, and a new 13- year species, Magicicada neotredecim. Evolution 54: 1313-1325. 
Martens J. 1996. Vocalizations and speciation of Palearctic birds. Pp 221-240 In: (D.E. Kroodsma \& E.H. Miller eds) Ecology and Evolution of Acoustic Communication in Birds. Cornell University Press, New York, U.S.A.

Mayr E. 1963. Animal Species and Evolution. Harvard University Press, Cambridge, Massachusetts. 798pp.

McGlone MS. 1988. New Zealand. Pp 557-599 In: (B Huntley \& T Webb III eds) Vegetation History. Kluwer Academic Publishers, Boston, U.S.A.

McGlone MS, Duncan RP, Heenan PB. 2001. Endemism, species selection and the origin and distribution of the vascular plant flora of New Zealand. Journal of Biogeography 28: 199-216.

Moritz C, Schneider CJ, Wake DB. 1992. Evolutionary relationships within the Ensatina eschscholtzii complex confirm the ring species interpretation. Systematic Zoology 41: 273-291.

Pole M. 1994. The New Zealand flora - entirely long-distance dispersal? Journal of Biogeography 21: 625-635.

Posada D, Crandall KA. 1998. Modeltest: testing the model of DNA substitution. Bioinformatics 14: 817-818. 
Posada D, Crandall KA, Templeton AR. 2000. GeoDis: A program for the Cladistic Nested Analysis of the Geographical Distribution of Genetic Haplotypes. Molecular Ecology 9: 487-488.

Rice WR, Hostert EE. 1993. Laboratory experiments on speciation: What have we learned in 40 years? Evolution 47: 1637-1653.

Ridley M. 1993. Evolution. Blackwell Scientific Publications, Boston, U.S.A. 670pp.

Rokas A, Ladoukakis E, Zouros E. 2003. Animal mitochondrial DNA recombination revisited. Trends in Ecology and Evolution 18: 411-417.

Schwarz G. 1978. Estimating the dimension of a model. The Annals of Statistics 6:461-464.

Servedio MR. 2001. Beyond reinforcement: the evolution of premating isolation by direct selection on preferences and postmating, prezygotic incompatibilities. Evolution 55: 1909-1920.

Simon C, Frati F, Beckenback A, Crespi B, Liu H, Flook P. 1994. Evolution, weighting, and phylogenetic utility of mitochondrial gene sequences and a compilation of conserved PCR primers. Annals of the Entomological Society of America 87: 651-701. 
Simon C, Tang J, Dalwadi S, Staley G, Deniega J, Unnasch TR. 2000.

Genetic evidence for assortative mating between 13-year cicadas and sympatric "17-year cicadas with 13-year life cycles" provides support for allochronic speciation. Evolution 54: 1326-1336.

Smissen RD, Breitwieser I, Ward JM, McLenachan PA, Lockhart PJ. 2003. Use of ISSR profiles and ITS-sequences to study the biogeography of alpine cushion plants in the genus Raoulia (Asteraceae). Plant Systematics and Evolution 239: 79-94.

Stöckler K, Daniel IL, Lockhart PJ. 2002. New Zealand Kauri (Agathis australis (D.Don) Lindl. Araucariaceae) survives Oligocene Drowning. Systematic Biology 51: 827-832.

Sutherland R. 1994. Displacement since the Pliocene along the southern section of the Alpine fault, New Zealand. Geology 22: 327-330.

Sutherland R. 1999. Cenozoic bending of New Zealand basement terranes and Alpine Fault displacement: a brief review. New Zealand Journal of Geology and Geophysics 42: 295-301.

Templeton AR. 1998. Nested clade analysis of phylogeographic data: testing hypothese about gene flow and population history. Molecular Ecology 7: 381-397. 
Templeton AR. 2001. Using phylogeographic analyses of gene trees to test species status and processes. Molecular Ecology 10: 779-791.

Templeton AR. 2004. Statistical phylogeography: methods of evaluating and minimizing inference errors. Molecular Ecology 13: 789-809.

Templeton AR, Boerwinkle E, Sing CF. 1987. A cladistic analysis of phenotypic associations with haplotypes inferred from restriction endonuclease mapping and DNA sequence data. I. Basic theory and an analysis of alcohol dehydrogenase activity in Drosophila. Genetics 117: 343-351.

Templeton AR, Crandall KA, Sing CF. 1992. A cladistic analysis of phenotypic associations with haplotypes inferred from restriction endonuclease mapping and DNA sequence data. III. Cladogram estimation. Genetics 132: 619-633.

Templeton AR, Routman E, Phillips CA. 1995. Separating population structure from population history: a cladistic analysis of the geographical distribution of mitochondrial DNA haplotypes in the tiger salamander, Ambystoma tigrinum. Genetics 149: 767-782.

Templeton AR, Sing CF. 1993. A cladistic analysis of phenotypic associations with haplotypes inferred from restriction endonuclease mapping. IV. Nested analyses with cladogram uncertainty and recombination. Genetics 134: 659-669. 
Thornton J. 1994. The Reed field guide to New Zealand geology : an introduction to rocks, minerals and fossils. Reed, Auckland, New Zealand. 226pp.

Trewick SA, Wallis GP. 2001. Bridging the "Beech-gap": New Zealand Invertebrate Phylogeography implicates Pleistocene Glaciation and Pliocene Isolation. Evolution 55: 2170-2180.

Trewick SA, Wallis GP, Morgan Richards M. 2000. Phylogeographical pattern correlates with Pliocene mountain building in the alpine scree weta (Orthoptera: Anostostomatidae). Molecular Ecology 9: 657-666.

Waaje JK. 1979. Reproductive character displacement in Calopteryx (Odonata: Calopterygidae). Evolution 33: 104-116.

Wake DB. 1997. Incipient species formation in salamanders of the Ensatina complex. Proceedings of the National Academy of Sciences USA 94: 7761-7767.

Wardle P. 1963. Evolution and distribution of the New Zealand flora, as affected by Quaternary climates. New Zealand Journal of Botany 1: 317.

Willet RW. 1950. The New Zealand Pleistocene snow line, climatic conditions, and suggested biological effects. New Zealand Journal of Science and Technology 32: 18-48. 
Williams K, Simon C. 1995. The ecology, behavior, and evolution of periodical cicadas. Annual Review of Entomology 40: 269-295.

Winkworth RC, Wagstaff SJ, Glenny D, Lockhart PJ. 2002. Plant dispersal N.E.W.S from New Zealand. Trends in Ecology \& Evolution 17: 514-520. 


\section{Appendix 2.1 Additional information on molecular labwork methods.}

Genomic DNA Extraction: The cicada specimen was removed from the EtOH and placed on a sterile surface. Using sterile forceps, the head and prothorax of the cicada was removed, and then part of the muscle tissue (approx 0.1g) within the mesothorax was pulled out and placed into a $1.5 \mathrm{~mL}$ microcentrifuge tube containing $0.5 \mathrm{~mL} \mathrm{ddH} \mathrm{H}_{2} \mathrm{O}$. After five minutes the $\mathrm{ddH}_{2} \mathrm{O}$ was carefully removed with a pipette leaving the rehydrated muscle tissue within the tube.

Three methods of extraction were trialled on two cicada specimens each: 1. the salting out method used by Buckley et al. 2001; 2. phenol chloroform; and 3. the Qiagen DNeasy ${ }^{\mathrm{TM}}$ Tissue Kit. Timing and safety considerations led to the selection of method 3 for the remainder of the specimens.

PCR amplification: 1. Qiagen Taq DNA Polymerase enzyme and solutions; 2. Applied Biosystems (Perkin Elmer) Gene Amp Amplitaq Gold enzyme and solutions; and 3. Applied Biosystems Amplitaq Gold Master Mix were trialled for the amplification of the ATP gene region. Both methods 1. and 3. were used.

3. For the Amplitaq Gold enzyme and solutions method, specimens were initially amplified on a MJ Research Peltier Thermal Cycler PTC-200 with an annealing temperature gradient across the block from $56-66^{\circ} \mathrm{C}$. After viewing the results on a $1 \%$ Agarose gel, an annealing temperature of $63^{\circ} \mathrm{C}$ was used for the rest of the A6-A8 amplifications using this method, and they were run on the same PCR machine. The Amplitaq Gold Master Mix method was run using the two-step method with a ten min initial hold on an Applied Biosystems GeneAmp PCR System 2700. 
PCR Amplification regimes:

PCR Amplification of 3' end of the COI gene

PCR reagents

$\mathrm{dd} \mathrm{H}_{2} \mathrm{O}$

$Q$ Solution

10x Buffer

dNTP

Primer 1 (C1-J-2195)

Primer 2 (TL2-N-3014)

Qiagen Taq enzyme

Genomic DNA

Mineral oil
Cycle Sequencing Protocol

$10.35 \mu \mathrm{L}$

$5.00 \mu \mathrm{L}$

$2.50 \mu \mathrm{L}$

$1.00 \mu \mathrm{L}$

$2.00 \mu \mathrm{L}$

$2.00 \mu \mathrm{L}$

$0.15 \mu \mathrm{L}$

$2.00 \mu \mathrm{L}$

$15 \mu \mathrm{L}$ $94^{\circ} \mathrm{C} \quad 2$ mins

$94^{\circ} \mathrm{C} \quad 45$ secs

$56^{\circ} \mathrm{C} \quad 45$ secs

$72^{\circ} \mathrm{C} 1 \min 15$ secs

$72{ }^{\circ} \mathrm{C} \quad 10$ mins

\section{PCR Amplification of the A6-A8 gene region}

1) Amplitaq Gold Enzyme and Solutions

PCR reagents

$\mathrm{dd}_{2} \mathrm{O}$

10x PCR Buffer

$\mathrm{MgCl}_{2}$

dNTP

Primer 1 (TK-J-3799)

Primer 2 (A6-N-4570)

AmpliTaq Gold enzyme

Genomic DNA
Cycle Sequencing Protocol

$14.42 \mu \mathrm{L}$

$2.40 \mu \mathrm{L}$

$2.40 \mu \mathrm{L}$

$2.00 \mu \mathrm{L}$

$1.00 \mu \mathrm{L}$

$1.00 \mu \mathrm{L}$

$0.28 \mu \mathrm{L}$

$1.50 \mu \mathrm{L}$ $94^{\circ} \mathrm{C} \quad 2$ mins

$94^{\circ} \mathrm{C} \quad 45$ secs

$64^{\circ} \mathrm{C} \quad 45$ secs

$72^{\circ} \mathrm{C} 1 \mathrm{~min} 15$ secs

$72{ }^{\circ} \mathrm{C} \quad 10$ mins

2) Amplitaq Gold Master Mix

PCR reagents

$\mathrm{ddH}_{2} \mathrm{O}$

Amplitaq Gold Master Mix

Primer 1 (TK-J-3799)

Primer 2 (A6-N-4570)

Genomic DNA
Cycle Sequencing 2-Step Protocol

$7.50 \mu \mathrm{L}$

$95^{\circ} \mathrm{C} \quad 10$ mins

$95^{\circ} \mathrm{C} \quad 15$ secs

$64^{\circ} \mathrm{C} 1 \mathrm{~min}$

$72^{\circ} \mathrm{C} \quad 7$ mins 


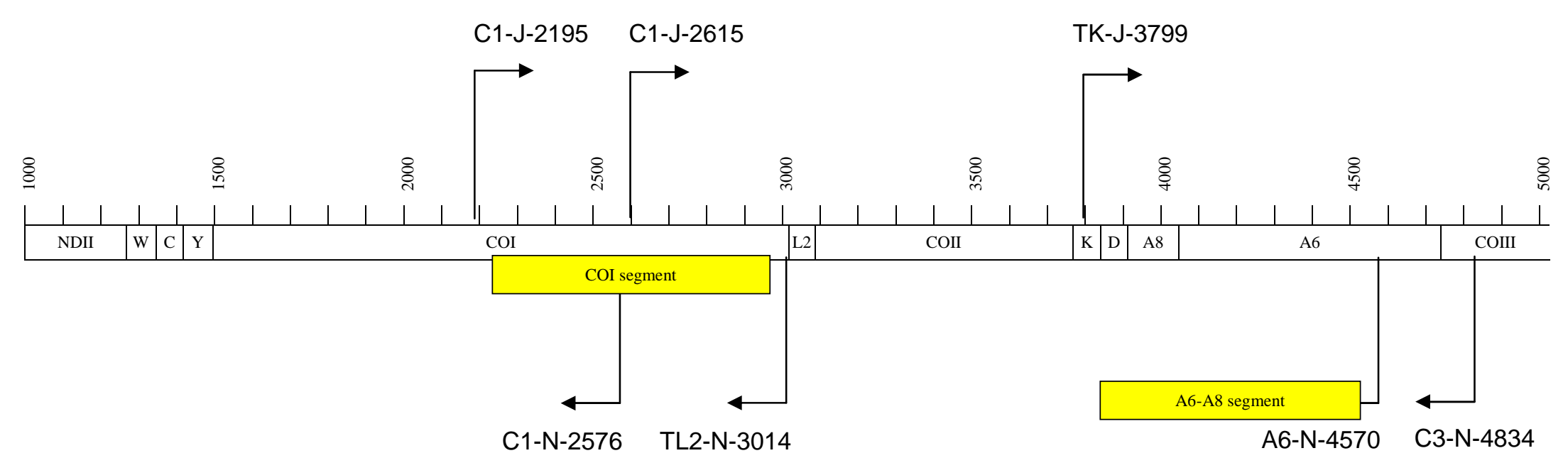

Appendix B. Map of part of insect mitochondrion. Locations of primers and areas sequenced for Maoricicada campbelli shown. 


\section{Appendix 3.1 Additional information on molecular labwork results.}

Genomic DNA Extraction: Two cicadas were extracted using the phenolchloroform method, two using the salting out method, and 184 using the Qiagen DNEasy tissue Kit. The DNEasy Kit was used as it was quick, safe and the DNA products are believed to have a good storage life. All extractions were visualised on a gel prior to PCR amplification; the first elution was used as the amplification template as there was a greater amount of DNA present.

PCR amplification and purification: The amplification of the COI region, whole and internal, was simple and effective for almost all of the M. campbelli. Occasionally an individual had to be re-amplified after viewing the gel, if the band was estimated to be too dim to give good sequencing results. The amplification of A6-A8 was more problematic. Many individuals had to be reamplified, and the results from two PCRs combined and purified together, to give enough DNA for sequencing. An MJResearch Thermal Cycler was used to optimise the annealing temperature for A6-A8 PCR reactions. Curiously, the result only worked for two of the temperatures attempted, and not for consecutive temperatures. The higher of the two, $63^{\circ} \mathrm{C}$ was chosen. If the amplification was still problematic, the C3-N-4834 primer used to obtain amplification. This primer was only used towards the end of the molecular labwork, and may actually be a better primer for this region.

PCR products were visualised on a gel prior to purification, and only purified if there was estimated to be enough DNA for sequencing. The final quantity of the elution buffer was estimated from the brightness of the PCR band; for A6A8 usually only $10 \mu \mathrm{L}$ was used, for COI usually $20 \mu \mathrm{L}$ of elution buffer was used.

Sequencing and alignment: $A$ total of $753 \mathrm{bp}$ of the $\mathrm{CO}$ region and $767 \mathrm{bp}$ of the A6-A8 region were aligned and edited. Only sequence clean of ambiguities was used. Because of the length of the amplified region, 20-200bp of sequence at the beginning and end of most alignments was single stranded. If a single stranded end was not a perfectly clean read, the segment was re-sequenced; this was rare, however, if the beginning was unreadable usually the rest of the 
sequence was too. Several individuals showed characteristic double peaks throughout their sequence that were presumed to be a large numt. This has been seen in other Maoricicada mtDNA sequence (T. Buckley pers. comm.) and in other cicada mtDNA sequence ( $C$. Simon pers. comm.). If the sequence was shown to have double peaks, additional sequence was obtained using the internal primers. The internal primers tended to amplify the mtDNA better than the presumed numt, so the sequence could be determined. If the sequence could not be determined from the internal primers, the individual was not added to the analysis. 
Appendix 3.2 Alignment of 71 Maoricicada campbelli haplotypes and two outgroups (Maoricicada clamitans (M.clamit) and Maoricicada phaeoptera (M phaeop) for 753bp of COI and 767bp of A6-A8. Blocks of haplotypes are shaded for ease of reading. The current gene is shown at the top of the page. Codons shaded black are those where the amino acid changes. The current number of bases is shown on the top right corner of each page. Note that codons in A8 are not aligned to spacing. 
Appendix: Halpotype sequences

COI

[60]

BSA1bsa1

TTA ATT TCT CAT ATT ATT ACA CAA GAA AGA GGT AAA ATT GAA TCC TTT GGT TCA TTA GGG

BSA1bsa2

BSA1bsa3

BSA1bsa 4

BSA1bsa5

BSA2bsa1

BSA3bsa1

BSA4bsa1

BSA5bsa1

BSA6bsa6

BSA7bsa11

BSA8bsa1

BSB1bsb1

BSB1bsb2

BSB1bsb3

BSB2bsb1

BSB3bsb1

BSB4bsb1

BSB5bsa7

BSC1bsa1

BSC2bsa1

BSC2bsa 8

BSC3bsa9

BSC4bsa10

BSC5bsa1

BSC6bsa1

BW1bw1

BW1bw3

BW1bw5

BW2bw1

BW2bw3

BW3bw2

BW4bw1

BW5bw4

BW6bw1

BW7bw1

BW8bw6

BW9bw7

BW10bw2

BM1bm1

BM1bm2

BM1bm3

BM1bm4

BM2bm1

BM2bm5

BM3bm1

BM4bm1

BM5bm2

BM6bm6

BN1bn1

BN1bn2

BN2bn1

BN3bn1

BK1bk1

BK2bk2

A1a1

$\begin{array}{llllllllllllllllllll}\ldots & \ldots & \ldots & \ldots & \ldots & \ldots & \ldots & \ldots & \ldots & \ldots & \ldots & \ldots & \ldots & \ldots & \ldots & \ldots & \ldots & \ldots & \ldots & \ldots \\ \ldots & \ldots & \ldots & \ldots & \ldots & \ldots & \ldots & \ldots & \ldots & \ldots & \ldots & \ldots & \ldots & \ldots & \ldots & \ldots & \ldots & \ldots & \ldots & \ldots\end{array}$

$\ldots \ldots+\ldots$

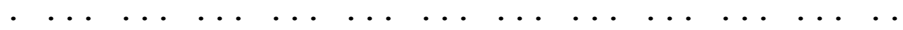

$\cdots \cdot$

.G

(1)

$\ldots \begin{array}{lllllllllllllllllll}\ldots & \ldots & \ldots & \ldots & \ldots & \ldots & \ldots & \ldots & \ldots & \ldots & \ldots & \ldots & \ldots & \ldots & \ldots & \ldots & \ldots & \ldots & \ldots\end{array}$

A2a1

A3a1

A4a2

CN1cn1

CN1cn2

CN1cn3

CN1cs1

CN2cn1

CN3cn 2

CN4Cn2

CS1cs1

CS1cs2

CS2cs2

CS3cs 1

CS4cs2

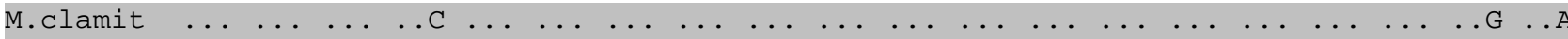

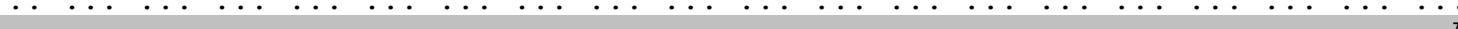

$\begin{array}{llllllllllllllllllll}\ldots & \ldots & \ldots & \ldots & \ldots & \ldots & \ldots & \ldots & \ldots & \ldots & \ldots & \ldots & \ldots & \ldots & \ldots & \ldots & \ldots & \ldots & \ldots & \ldots A \\ \ldots & \ldots & \ldots & \ldots & \ldots & \ldots & \ldots & \ldots & \ldots & \ldots & \ldots & \ldots & \ldots & \ldots & \ldots & \ldots & \ldots & \ldots & \ldots & \ldots A\end{array}$

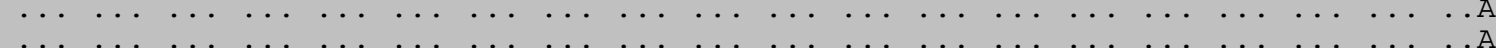

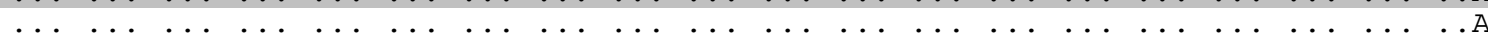

$\begin{array}{lllllllllllllllllllll}\ldots & \ldots & \ldots & \ldots & \ldots & \ldots & \ldots & \ldots & \ldots & \ldots & \ldots & \ldots & \ldots & \ldots & \ldots & \ldots & \ldots & \ldots & \ldots & \ldots A\end{array}$

$\begin{array}{lllllllllllllllllll}\ldots & \ldots & \ldots & \ldots & \ldots & \ldots & \ldots & \ldots & \ldots & \ldots & \ldots & \ldots & \ldots & \ldots & \ldots & \ldots & \ldots & \ldots & \ldots\end{array}$

$\begin{array}{llllllllllllllllllll}\ldots & \ldots & \ldots & \ldots & \ldots & \ldots & \ldots & \ldots & \ldots & \ldots & \ldots & \ldots & \ldots & \ldots & \ldots & \ldots & \ldots & \ldots & \ldots & \ldots A \\ \ldots & \ldots & \ldots & \ldots & \ldots & \ldots & \ldots & \ldots & \ldots & \ldots & \ldots & \ldots & \ldots & \ldots & \ldots & \ldots & \ldots & \ldots & \ldots & \ldots A\end{array}$

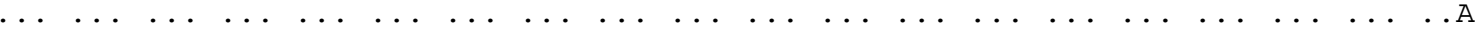

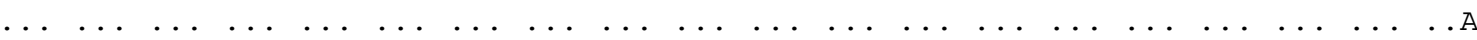

$\begin{array}{lllllllllllllllllllllll}\ldots & \ldots & \ldots & \ldots & \ldots & \ldots & \ldots & \ldots & \ldots & \ldots & \ldots & \ldots & \ldots & \ldots & \ldots & \ldots & \ldots & \ldots & \ldots & \ldots A\end{array}$

$\ldots \ldots \ldots \ldots \ldots \ldots$

$\begin{array}{llllllllllllllllllllll}\ldots & \ldots & \ldots & \ldots & \ldots & \ldots & \ldots & \ldots & \ldots & \ldots & \ldots & \ldots & \ldots & \ldots & \ldots & \ldots & \ldots & \ldots & \ldots & \ldots A\end{array}$

$\ldots \begin{array}{llllllllllllllllllll}\ldots & \ldots & \ldots & \ldots & \ldots & \ldots & \ldots & \ldots & \ldots & \ldots & \ldots & \ldots & \ldots & \ldots & \ldots & \ldots & \ldots & \ldots & \ldots G & \ldots A\end{array}$

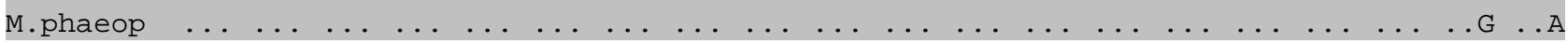


Appendix D: Halpotype sequences

COI

[120]

BSA1bsa1

ATA ATC TAT GCT ATA ATA TCA ATT GGT ATT TTA GGA TTT GTT GTA TGg GCA CAT CAT ATA

BSA1bsa2

BSA1bsa3

BSA1bsa 4

BSA1bsa5

BSA2bsa1

BSA3bsa1

BSA4bsa1

BSA5bsa1

BSA6bsa 6

BSA7bsa11

BSA8bsa1

BSB1bsb1

BSB1bsb2

BSB1bsb3

BSB2bsb1

BSB3bsb1

BSB4bsb1

BSB5bsa 7

BSC1bsa1

BSC2bsa1

BSC2bsa8

BSC3bsa9

BSC4bsa10

BSC5bsa1

BSC6bsa1

BW1bw1

BW1bw3

BW1bw5

BW2bw1

BW2bw3

BW3bw2

BW4bw1

BW5bw4

BW6bw1

BW7bw1

BW8bw6

BW9bw7

BW10bw2

BM1bm1

BM1bm2

BM1bm3

BM1bm4

BM2bm1

BM2bm5

BM3bm1

BM4bm1

BM5bm2

BM6bm6

BN1bn1

BN1bn2

BN2bn1

BN3bn1

BK1bk1

BK2bk2

A1a1

A2a1

A3a1

A4a2

CN1Cn1

CN1Cn2

CN1Cn3

CN1CS1

CN2Cn1

CN3Cn2

CN4Cn2

CS1cs1

CS1cs2

CS2cs2

CS3cs 1

M.clamit

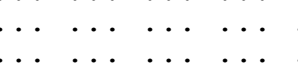

$\ldots$

$\ldots \ldots$

.

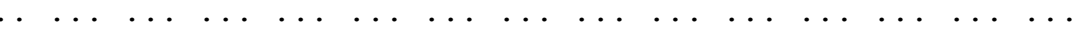

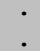

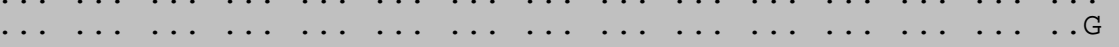

$\cdots$

$\ldots$

$\cdots$

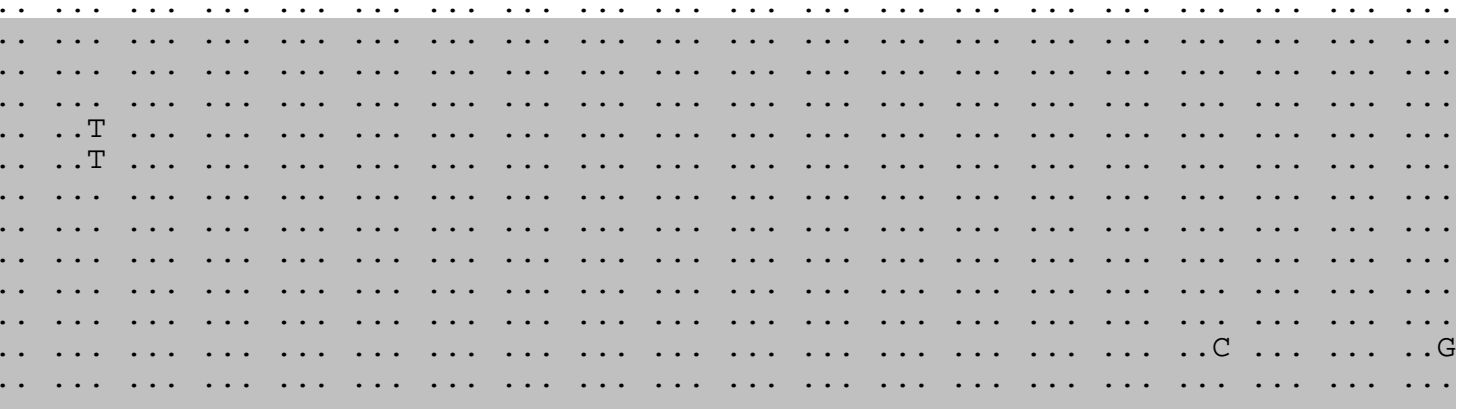

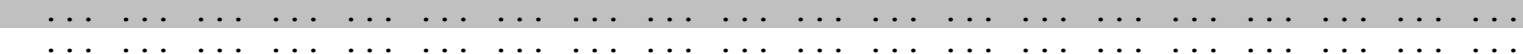

M. phaeop

$\begin{array}{llllllllllllllllllll}\ldots & \ldots & \ldots & \ldots & \ldots & \ldots & \ldots & \ldots & \ldots & \ldots & \ldots & \ldots & \ldots & \ldots & \ldots G & \ldots & \ldots & \ldots & \ldots & \ldots \\ \ldots & \ldots & \ldots & \ldots & \ldots & \ldots & \ldots & \ldots & \ldots & \ldots & \ldots & \ldots & \ldots & \ldots & \ldots G & \ldots & \ldots & \ldots & \ldots & \ldots \\ \ldots & \ldots & \ldots & \ldots & \ldots & \ldots & \ldots & \ldots & \ldots & \ldots & \ldots & \ldots & \ldots & \ldots & \ldots G & \ldots & \ldots & \ldots & \ldots & \ldots \\ \ldots & \ldots T & \ldots & \ldots & \ldots & \ldots & \ldots & \ldots & \ldots & \ldots & \ldots & \ldots & \ldots & \ldots & \ldots G & \ldots & \ldots & \ldots & \ldots & \ldots \\ \ldots & \ldots T & \ldots & \ldots & \ldots & \ldots & \ldots & \ldots & \ldots & \ldots & \ldots & \ldots & \ldots & \ldots & \ldots G & \ldots & \ldots & \ldots & \ldots & \ldots G \\ \ldots & \ldots T & \ldots & \ldots & \ldots & \ldots & \ldots & \ldots & \ldots & \ldots & \ldots & \ldots & \ldots & \ldots & \ldots G & \ldots & \ldots & \ldots & \ldots & \ldots G \\ \ldots & \ldots T & \ldots & \ldots & \ldots & \ldots & \ldots & \ldots & \ldots & \ldots & \ldots & \ldots & \ldots & \ldots & \ldots G & \ldots & \ldots & \ldots & \ldots & \ldots G \\ \ldots & \ldots T & \ldots & \ldots & \ldots & \ldots & \ldots & \ldots & \ldots & \ldots & \ldots & \ldots & \ldots & \ldots & \ldots G & \ldots & \ldots & \ldots & \ldots & \ldots G \\ \ldots & \ldots T & \ldots & \ldots & \ldots & \ldots & \ldots & \ldots & \ldots & \ldots & \ldots & \ldots & \ldots & \ldots & \ldots G & \ldots & \ldots & \ldots & \ldots & \ldots \\ \ldots & \ldots T & \ldots & \ldots & \ldots & \ldots & \ldots & \ldots & \ldots & \ldots & \ldots & \ldots & \ldots & \ldots & \ldots G & \ldots & \ldots & \ldots & \ldots & \ldots G \\ \ldots & \ldots T & \ldots & \ldots & \ldots & \ldots & \ldots & \ldots & \ldots & \ldots & \ldots & \ldots & \ldots & \ldots & \ldots G & \ldots & \ldots & \ldots & \ldots & \ldots G \\ \ldots & \ldots T & \ldots & \ldots & \ldots & \ldots & \ldots & \ldots & \ldots & \ldots & \ldots & \ldots & \ldots & \ldots & \ldots G & \ldots & \ldots & \ldots & \ldots & \ldots G \\ \ldots & \ldots T & \ldots & \ldots & \ldots & \ldots & \ldots & \ldots & \ldots & \ldots & \ldots & \ldots & \ldots & \ldots & \ldots G & \ldots & \ldots & \ldots & \ldots & \ldots G \\ \ldots & \ldots T & \ldots & \ldots & \ldots & \ldots & \ldots & \ldots & \ldots & \ldots & \ldots & \ldots & \ldots & \ldots & \ldots G & \ldots & \ldots & \ldots & \ldots & \ldots G \\ \ldots & \ldots T & \ldots & \ldots & \ldots & \ldots & \ldots & \ldots & \ldots & \ldots & \ldots & \ldots & \ldots & \ldots & \ldots G & \ldots & \ldots & \ldots & \ldots & \ldots G \\ \ldots & \ldots T & \ldots & \ldots & \ldots & \ldots & \ldots & \ldots & \ldots & \ldots & \ldots & \ldots & \ldots & \ldots & \ldots G & \ldots & \ldots & \ldots & \ldots & \ldots \\ \ldots & \ldots T & \ldots & \ldots & \ldots & \ldots & \ldots & \ldots & \ldots & \ldots & \ldots & \ldots & \ldots & \ldots & \ldots G & \ldots & \ldots & \ldots & \ldots & \ldots G\end{array}$


Appendix D: Halpotype sequences

COI

BSA1bsa1

TTT ACT GTA GGT ATA GAT GTT GAT ACA CGT GCA TAT TTT ACA TCA GCT ACT ATA ATT ATT

BSA1bsa2

BSA1bsa3

BSA1bsa 4

BSA1bsa5

BSA2bsa1

BSA3bsa1

BSA4bsa1

BSA5bsa1

BSA6bsa 6

BSA7bsa11

BSA8bsa1

BSB1bsb1

BSB1bsb2

BSB1bsb3

BSB2bsb1

BSB3bsb1

BSB4bsb1

BSB5bsa 7

BSC1bsa1

BSC2bsa1

BSC2bsa8

BSC3bsa9

BSC4bsa10

BSC5bsa1

BSC6bsa1

$\begin{array}{llllllllllllllllllll}\ldots & \ldots & \ldots & \ldots & \ldots & \ldots & \ldots & \ldots & \ldots & \ldots & \ldots & \ldots & \ldots & \ldots & \ldots & \ldots & \ldots & \ldots & \ldots & \ldots \\ \ldots & \ldots & \ldots & \ldots & \ldots & \ldots & \ldots & \ldots & \ldots & \ldots & \ldots & \ldots & \ldots & \ldots & \ldots & \ldots & \ldots & \ldots & \ldots & \ldots\end{array}$

$\ldots \ldots$

$\begin{array}{lllllllllllll} & \ldots & \ldots & \ldots & \ldots & \ldots & \ldots & \ldots & \ldots & \ldots & \ldots & \ldots & \ldots\end{array}$

$\ldots$

$\cdots \cdots$

.

BW1bw1

BW1bw3

BW1bw5

BW2bw1

BW2bw3

BW3bw2

BW4bw1

BW5bw4

BW6bw1

BW7bw1

BW8bw6

BW9bw7

BW10bw2

BM1bm1

BM1bm2

BM1bm3

BM1bm4

BM2bm1

BM2bm5

BM3bm1

BM4bm1

BM5bm2

BM6bm6

BN1bn1

BN1bn2

BN2bn1

BN3bn1

BK1bk1

BK2bk2

A1a1

A2a1

A3a1

A4a2

CN1Cn1

CN1Cn2

CN1Cn3

CN1CS1

CN2Cn1

CN3Cn 2

CN4Cn2

CS1CS1

CS1cs2

CS2cs2

CS3cs 1

CS4cs2 $\mathrm{G}$

$\begin{array}{lllll}\cdots & \ldots & \ldots & \ldots & \ldots G\end{array}$

M. clamit

M. phaeop

$\begin{array}{lllllllllllllllllllll}\ldots & \ldots & \ldots & \ldots & \ldots & \ldots & \ldots & \ldots & \ldots & \ldots & \ldots & \ldots & \ldots & \ldots & \ldots & \ldots & \ldots & \ldots & \ldots & \ldots \\ \ldots & \ldots & \ldots & \ldots & \ldots G & \ldots C & \ldots & \ldots & \ldots G & \ldots & \ldots & \ldots & \ldots & \ldots & \ldots & \ldots & \ldots & \ldots & \ldots & \ldots \\ \ldots & \ldots & \ldots & \ldots & \ldots G & \ldots C & \ldots & \ldots & \ldots G & \ldots & \ldots & \ldots & \ldots & \ldots & \ldots & \ldots & \ldots & \ldots & \ldots & \ldots \\ \ldots & \ldots & \ldots & \ldots & \ldots G & \ldots C & \ldots & \ldots & \ldots G & \ldots & \ldots & \ldots & \ldots & \ldots & \ldots & \ldots & \ldots & \ldots & \ldots & \ldots \\ \ldots & \ldots & \ldots & \ldots & \ldots G & \ldots C & \ldots & \ldots & \ldots G & \ldots & \ldots & \ldots & \ldots & \ldots & \ldots & \ldots & \ldots & \ldots & \ldots & \ldots \\ \ldots & \ldots & \ldots & \ldots & \ldots G & \ldots C & \ldots & \ldots & \ldots G & \ldots & \ldots & \ldots & \ldots & \ldots & \ldots & \ldots & \ldots & \ldots & \ldots & \ldots \\ \ldots & \ldots & \ldots & \ldots & \ldots G & \ldots C & \ldots & \ldots & \ldots G & \ldots & \ldots & \ldots & \ldots & \ldots & \ldots & \ldots & \ldots & \ldots & \ldots & \ldots \\ \ldots & \ldots & \ldots & \ldots & \ldots G & \ldots C & \ldots & \ldots & \ldots G & \ldots & \ldots & \ldots & \ldots & \ldots & \ldots & \ldots & \ldots & \ldots & \ldots & \ldots \\ \ldots & \ldots & \ldots & \ldots & \ldots G & \ldots C & \ldots & \ldots & \ldots G & \ldots & \ldots & \ldots & \ldots & \ldots & \ldots & \ldots & \ldots & \ldots & \ldots & \ldots \\ \ldots & \ldots & \ldots & \ldots & \ldots G & \ldots C & \ldots & \ldots & \ldots & \ldots & \ldots & \ldots & \ldots & \ldots & \ldots & \ldots & \ldots & \ldots & \ldots & \ldots & \ldots \\ \ldots & \ldots & \ldots & \ldots & \ldots G & \ldots C & \ldots & \ldots & \ldots & \ldots & \ldots & \ldots & \ldots & \ldots & \ldots & \ldots & \ldots & \ldots & \ldots & \ldots \\ \ldots & \ldots & \ldots & \ldots A & \ldots & \ldots C & \ldots & \ldots C & \ldots & \ldots & \ldots & \ldots & \ldots & \ldots & \ldots & \ldots & \ldots & \ldots & \ldots & \ldots \\ \ldots & \ldots & \ldots & \ldots C & \ldots & \ldots C & \ldots & \ldots & \ldots & \ldots & \ldots & \ldots & \ldots & \ldots & \ldots & \ldots & \ldots & \ldots & \ldots & \ldots \\ \end{array}$


Appendix D: Halpotype sequences

COI

BSA1bsa1

BSA1bsa2

BSA1bsa3

BSA1bsa 4

BSA1bsa5

BSA2bsa1

BSA3bsa1

BSA4bsa1

BSA5bsa1

BSA6bsa6

BSA7bsa11

BSA8bsa1

BSB1bsb1

BSB1bsb2

BSB1bsb3

BSB2bsb1

BSB3bsb1

BSB4bsb1

BSB5bsa7

BSC1bsa1

BSC2bsa1

BSC2bsa8

BSC3bsa9

BSC4bsa10

BSC5bsa1

BSC6bsa1

BW1bw1

BW1bw3

BW1bw5

BW2bw1

BW2bw3

BW3bw2

BW4bw1

BW5bw4

BW6bw1

BW7bw1

BW8bw6

BW9bw7

BW10bw2

BM1bm1

BM1bm2

BM1bm3

BM1bm4

BM2bm1

BM2bm5

BM3bm1

BM4bm1

BM5bm2

BM6bm6

BN1bn1

BN1bn2

BN2bn1

BN3bn1

BK1bk1

BK2bk2

A1a1

A2a1

A3a1

A4a2

CN1Cn1

CN1Cn2

CN1Cn3

CN1cs1

CN2Cn1

CN3cn 2

CN4Cn2

CS1cs1

CS1cs 2

CS2cs2

CS3cs 1

CS4cs2

M.clamit

M. phaeop
GCA GTT CCA ACT GGA ATT AAA GTG TTC AGT TGA CTT GCA ACT TTA AGA GgA ATA AAA ATT

$\begin{array}{llllllllllllllllllll}\ldots & \ldots & \ldots & \ldots & \ldots & \ldots & \ldots & \ldots & \ldots & \ldots & \ldots & \ldots & \ldots & \ldots & \ldots & \ldots & \ldots & \ldots & \ldots & \ldots \\ \ldots & \ldots & \ldots & \ldots & \ldots & \ldots & \ldots & \ldots & \ldots & \ldots & \ldots & \ldots & \ldots & \ldots & \ldots & \ldots & \ldots & \ldots & \ldots & \ldots\end{array}$

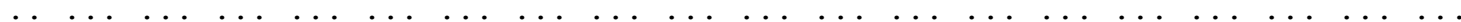

$\begin{array}{lllllllllllllllllll}\cdots & \cdots & \cdots & \cdots & \cdots & \cdots & \cdots & \cdots & \cdots & \cdots & \cdots & \cdots & \cdots & \cdots & \cdots & \cdots & \cdots & \cdots & \cdots\end{array}$

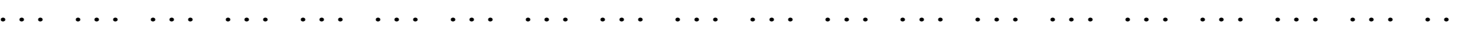

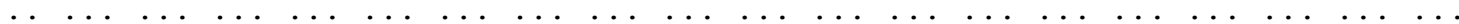

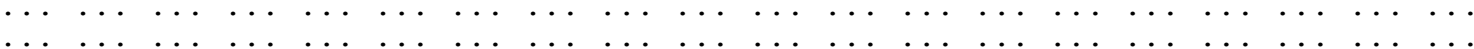

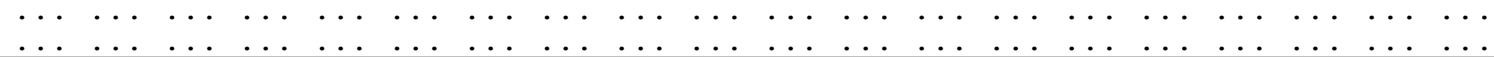

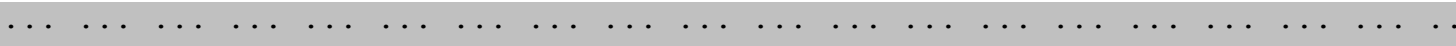

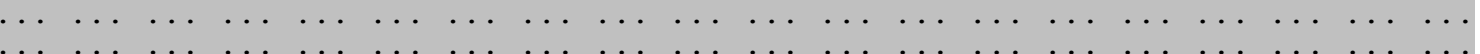

$\begin{array}{llllllllllllllllllll}\ldots & \ldots & \ldots & \ldots & \ldots & \ldots & \ldots & \ldots & \ldots & \ldots & \ldots & \ldots & \ldots & \ldots & \ldots & \ldots & \ldots & \ldots & \ldots & \ldots \\ \ldots & \ldots & \ldots & \ldots & \ldots & \ldots & \ldots & \ldots & \ldots & \ldots & \ldots & \ldots & \ldots & \ldots & \ldots & \ldots & \ldots & \ldots & \ldots & \ldots\end{array}$

$\begin{array}{llllllllllllllllllllll}\ldots & \ldots & \ldots & \ldots & \ldots & \ldots & \ldots & \ldots & \ldots & \ldots & \ldots & \ldots & \ldots & \ldots & \ldots & \ldots & \ldots & \ldots & \ldots & \ldots \\ \ldots & \ldots & \ldots & \ldots & \ldots & \ldots & \ldots & \ldots & \ldots & \ldots & \ldots & \ldots & \ldots & \ldots & \ldots & \ldots & \ldots & \ldots & \ldots & \ldots\end{array}$

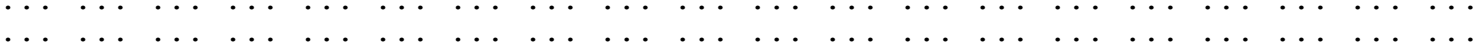

$\ldots \begin{array}{lllllllllllllllllll}\ldots & \ldots & \ldots & \ldots & \ldots & \ldots & \ldots & \ldots & \ldots & \ldots & \ldots & \ldots & \ldots & \ldots & \ldots & \ldots & \ldots & \ldots & \ldots \\ \ldots\end{array}$

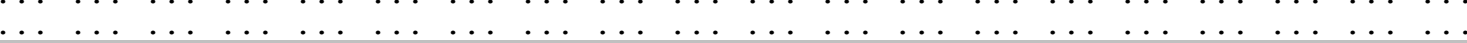


Appendix D: Halpotype sequences

COI

[300]

BSA1bsa1 AAT ATC ACA TCT TCA GCT TTA TGA GCC CTA GGA TTT GTT TTT TTA TTT ACA ATT GGT GGT

BSA1bsa2

BSA1bsa3

BSA1bsa 4

BSA1bsa5

BSA2bsa1

BSA3bsa1

BSA4bsa1

BSA5bsa1

BSA6bsa 6

BSA7bsa11

BSA8bsa1

BSB1bsb1

BSB1bsb2

BSB1bsb3

BSB2bsb1

BSB3bsb1

BSB4bsb1

BSB5bsa7

BSC1bsa1

BSC2bsa1

BSC2bsa8

BSC3bsa9

BSC4bsa10

BSC5bsa1

BSC6bsa1

BW1bw1

BW1bw3

BW1bw5

BW2bw1

BW2bw3

BW3bw2

BW4bw1

BW5bw4

BW6bw1

BW7bw1

BW8bw6

BW9bw7

BW10bw2

BM1bm1

BM1bm2

BM1bm3

BM1bm4

BM2bm1

BM2bm5

BM3bm1

BM4bm1

BM5bm2

BM6bm6

BN1bn1

BN1bn2

BN2bn1

BN3bn1

BK1bk1

BK2bk2

A1a1

A2a1

A3a1

A4a2

CN1cn1

CN1cn2

CN1cn3

CN1CS1

CN2Cn1

CN3Cn 2

CN4Cn2

CS1cs1

CS1cs 2

CS2cs2

CS3cs 1

CS4cs2

M. clamit

T..

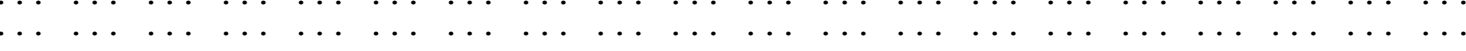

$\begin{array}{llllllllllllllllllllllll}\ldots & \ldots & \ldots & \ldots & \ldots & \ldots & \ldots & \ldots & \ldots & \ldots & \ldots & \ldots & \ldots & \ldots & \ldots & \ldots & \ldots & \ldots & \ldots & \ldots\end{array}$

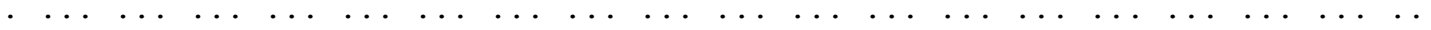

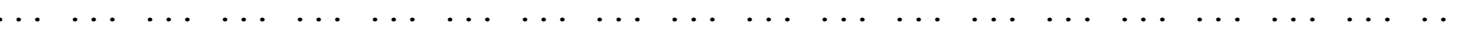

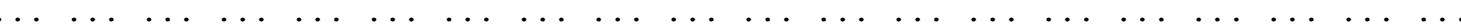

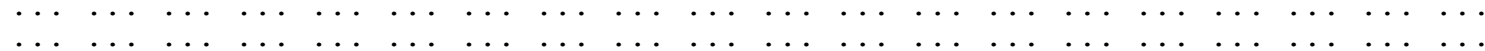

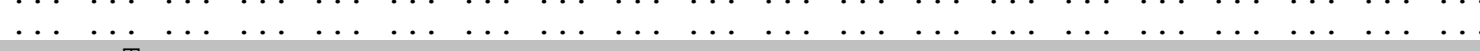

. . T

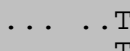

$\ldots, \ldots$

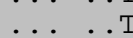

$\ldots$

$\ldots, \ldots, \ldots$

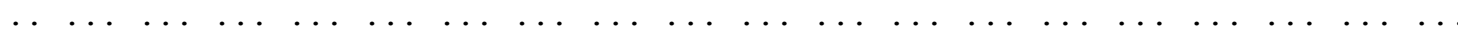

(1)

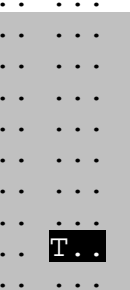

$\begin{array}{lllllllllll}\ldots & \ldots & \ldots & \ldots & \ldots & \ldots & \ldots & \ldots & \ldots & \ldots & \ldots \\ \ldots & \ldots & \ldots & \ldots & \ldots & \ldots & \ldots & \ldots & \ldots & \ldots & \ldots \\ \ldots & \ldots & \ldots & \ldots & \ldots & \ldots & \ldots & \ldots & \ldots & \ldots & \end{array}$

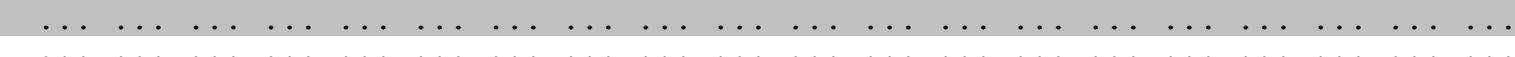

M. phaeop 
Appendix D: Halpotype sequences

COI

BSA1bsa1

TTA ACT GGA GTA ATT TTA GCT AAT TCT TCG ATT GAT ATT ATA TTA CAT GAT ACA TAT TAT

BSA1bsa2

BSA1bsa3

BSA1bsa 4

BSA1bsa5

BSA2bsa1

BSA3bsa1

BSA4bsa1

BSA5bsa1

BSA6bsa6

BSA7bsa11

BSA8bsa1

BSB1bsb1

BSB1bsb2

BSB1bsb3

BSB2bsb1

BSB3bsb1

BSB4bsb1

BSB5bsa7

BSC1bsa1

BSC2bsa1

BSC2bsa8

BSC3bsa9

BSC4bsa10

BSC5bsa1

BSC6bsa1

$\begin{array}{llllllllllllllllllll}\ldots & \ldots & \ldots & \ldots & \ldots & \ldots & \ldots & \ldots & \ldots & \ldots & \ldots & \ldots & \ldots & \ldots & \ldots & \ldots & \ldots & \ldots & \ldots & \ldots \\ \ldots & \ldots & \ldots & \ldots & \ldots & \ldots & \ldots & \ldots & \ldots & \ldots & \ldots & \ldots & \ldots & \ldots & \ldots & \ldots & \ldots & \ldots & \ldots & \ldots\end{array}$

$\ldots \ldots$

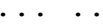

$\cdots \cdots$

.

.

A

BW1bw1

BW1bw3

BW1bw5

BW2bw1

BW2bw3

BW3bw2

BW4bw1

BW5bw4

BW6bw1

BW7bw1

BW8bw6

BW9bw7

BW10bw2

BM1bm1

BM1bm2

BM1bm3

BM1bm4

BM2bm1

BM2bm5

BM3bm1

BM4bm1

BM5bm2

BM6bm6

BN1bn1

BN1bn2

BN2bn1

BN3bn1

BK1bk1

BK2bk2

A1a1

A2a1

A3a1

A4a2

CN1Cn1

CN1Cn2

CN1Cn3

CN1Cs1

CN2Cn1

CN3Cn 2

CN4Cn2

CS1CS1

CS1cs2

CS2cs2

CS3cs 1

CS4cs2

M. clamit

M. phaeop

A

A

A 
Appendix D: Halpotype sequences

COI

[420]

BSA1bsa1

GTA GTT GCT CAT TTT CAT TAT GTT CTG TCA ATA GGA GCA GTT TTT GCT ATT ATG GCA AGA

BSA1bsa3

BSA1bsa4

BSA1bsa5

BSA2bsa1

BSA3bsa1

BSA4bsa1

BSA5bsa1

BSA6bsa6

BSA7bsa11

BSA8bsa1

BSB1bsb1

BSB1bsb2

BSB1bsb3

BSB2bsb1

BSB3bsb1

BSB4bsb1

BSB5bsa7

BSC1bsa1

BSC2bsa1

BSC2bsa8

BSC3bsa9

BSC4bsa10

BSC5bsa1

BSC6bsa1

BW1bw1

BW1bw3

BW1bw5

BW2bw1

BW2bw3

BW3bw2

BW4bw1

BW5bw4

BW6bw1

BW7bw1

BW8bw6

BW9bw7

BW10bw2

BM1bm1

BM1bm2

BM1bm3

BM1bm4

BM2bm1

BM2bm5

BM3bm1

BM4bm1

BM5bm2

BM6bm6

BN1bn1

BN1bn2

BN2bn1

BN3bn1

BK1bk1

BK2bk2

A1a1

A2a1

A3a1

A4a2

CN1Cn1

CN1Cn2

CN1Cn3

CN1CS1

CN2Cn1

CN3Cn2

CN4Cn2

CS1CS1

CS1cs2

CS2cs2

CS3cs 1

CS4cs2

$\begin{array}{llllllllllllllllllll}\ldots & \ldots & \ldots & \ldots & \ldots & \ldots & \ldots & \ldots & \ldots & \ldots & \ldots & \ldots & \ldots & \ldots & \ldots & \ldots & \ldots & \ldots & \ldots & \ldots \\ \ldots & \ldots & \ldots & \ldots & \ldots & \ldots & \ldots & \ldots & \ldots & \ldots & \ldots & \ldots & \ldots & \ldots & \ldots & \ldots & \ldots & \ldots & \ldots & \ldots \\ \ldots & \ldots & \ldots & \ldots & \ldots & \ldots & \ldots & \ldots & \ldots & \ldots & \ldots & \ldots & \ldots & \ldots & \ldots & \ldots & \ldots & \ldots & \ldots\end{array}$

$\begin{array}{lllllllllllll}\ldots & \ldots & \ldots & \ldots & \ldots & \ldots & \ldots & \ldots & \ldots & \ldots & \ldots & \ldots & \ldots \\ \ldots & \ldots & \ldots & \ldots & \ldots & \ldots & \ldots & \ldots & \ldots & \ldots\end{array}$

$\cdots, \cdots$

$\cdots$

$\ldots \ldots$

.

... . . . . . . .


Appendix D: Halpotype sequences

COI

[480]

BSA1bsa1

TTT ATT CAC TGA TTT CCT CTT TTT ACA GGA ATA ACA TTA AAT TCA AAT TGA TTA AAA ATT

BSA1bsa2

BSA1bsa3

BSA1bsa 4

BSA1bsa5

BSA2bsa1

BSA3bsa1

BSA4bsa1

BSA5bsa1

BSA6bsa6

BSA7bsa11

BSA8bsa1

BSB1bsb1

BSB1bsb2

BSB1bsb3

BSB2bsb1

BSB3bsb1

BSB4bsb1

BSB5bsa7

BSC1bsa1

BSC2bsa1

BSC2bsa8

BSC3bsa9

BSC4bsa10

BSC5bsa1

BSC6bsa1

BW1bw1

BW1bw3

BW1bw5

BW2bw1

BW2bw3

BW3bw2

BW4bw1

BW5bw4

BW6bw1

BW7bw1

BW8bw6

BW9bw7

BW10bw2

BM1bm1

BM1bm2

BM1bm3

BM1bm4

BM2bm1

BM2bm5

BM3bm1

BM4bm1

BM5bm2

BM6bm6

BN1bn1

BN1bn2

BN2bn1

BN3bn1

BK1bk1

BK2bk2

A1a1

A2a1

A3a1

A4a2

CN1cn1

CN1Cn2

CN1Cn3

CN1CS1

CN2Cn1

CN3Cn2

CN4Cn2

CS1CS1

CS1cs2

CS2cs2

CS3cs 1

CS4cs2

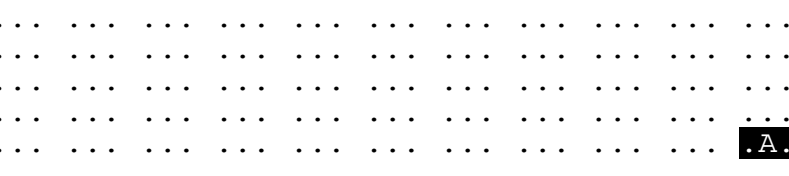

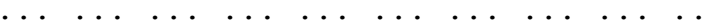

$\ldots+\ldots, \ldots$

A.

. 
Appendix D: Halpotype sequences

COI

BSA1bsa1

CAT TTT TTA TTA ATA TTT ATT GGT GTA AAC ATA ACA TTT TTT CCT CAA CAT TTC TTA GGA

BSA1bsa2

BSA1bsa3

BSA1bsa4

BSA1bsa5

BSA2bsa1

BSA3bsa1

BSA4bsa1

BSA5bsa1

BSA6bsa6

BSA7bsa11

BSA8bsa1

BSB1bsb1

BSB1bsb2

BSB1bsb3

BSB2bsb1

BSB3bsb1

BSB4bsb1

BSB5bsa7

BSC1bsa1

BSC2bsa1

BSC2bsa8

BSC3bsa9

BSC4bsa10

BSC5bsa1

BSC6bsa1

BW1bw1

BW1bw3

BW1bw5

BW2bw1

BW2bw3

BW3bw2

BW4bw1

BW5bw4

BW6bw1

BW7bw1

BW8bw6

BW9bw7

BW10bw2

BM1bm1

BM1bm2

BM1bm3

BM1bm4

BM2bm1

BM2bm5

BM3bm1

BM4bm1

BM5bm2

BM6bm6

BN1bn1

BN1bn2

BN2bn1

BN3bn1

BK1bk1

BK2bk2

A1a1

A2a1

A3a1

A4a2

CN1cn1

CN1Cn2

CN1Cn3

CN1CS1

CN2Cn1

CN3Cn2

CN4Cn2

CS1cs1

CS1cs2

CS2cs2

CS3cs 1

CS4cs2

M.clamit

M. phaeop

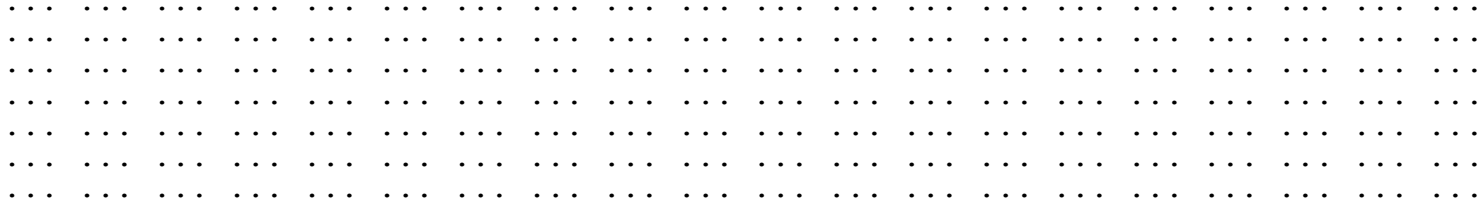


Appendix D: Halpotype sequences

COI

BSA1bsa1

CTA AGA GGT ATG CCT CGC CGA TAT TCA GAT TAT CCT GAC GCT TAT ATA TCA TGA AAT ATA

BSA1bsa2

BSA1bsa3

BSA1bsa4

BSA1bsa5

BSA2bsa1

BSA3bsa1

BSA4bsa1

BSA5bsa1

BSA6bsa6

BSA7bsa11

BSA8bsa1

BSB1bsb1

BSB1bsb2

BSB1bsb3

BSB2bsb1

BSB3bsb1

BSB4bsb1

BSB5bsa7

BSC1bsa1

BSC2bsa1

BSC2bsa8

BSC3bsa9

BSC4bsa10

BSC5bsa1

BSC6bsa1

BW1bw1

BW1bw3

BW2bw1

BW2bw3

BW3bw2

BW4bw1

BW5bw4

BW6bw1

BW7bw1

BW8bw6

BW9bw7

BW10bw2

BM1bm1

BM1bm2

BM1bm3

BM1bm4

BM2bm1

BM2bm5

BM3bm1

BM4bm1

BM5bm2

BM6bm6

BN1bn1

BN1bn2

BN2bn1

BN3bn1

BK1bk1

$\begin{array}{llllllllllllllllllll}\ldots & \ldots & \ldots & \ldots & \ldots & \ldots & \ldots & \ldots & \ldots & \ldots & \ldots & \ldots & \ldots & \ldots & \ldots & \ldots & \ldots & \ldots & \ldots & \ldots \\ \ldots & \ldots & \ldots & \ldots & \ldots & \ldots & \ldots & \ldots & \ldots & \ldots & \ldots & \ldots & \ldots & \ldots & \ldots & \ldots & \ldots & \ldots & \ldots & \ldots\end{array}$

$\ldots \ldots$

$\ldots$

$\cdots+\cdots$

$\ldots \ldots$

.

$\begin{array}{lllllllllll}\ldots & \ldots & \ldots & \ldots & \ldots & \ldots & \ldots & \ldots & \ldots & \ldots & \ldots \\ \ldots & \ldots & \ldots & \ldots & \ldots & \ldots & \ldots & \ldots & \ldots & \ldots & \ldots \\ \ldots & \ldots & \ldots & \ldots & \ldots & \ldots & \ldots & \ldots & \ldots & \ldots & \ldots \\ \ldots & \ldots & \ldots & \ldots & \ldots & \ldots & \ldots & \ldots & \ldots & \ldots & \ldots \\ \ldots & \ldots & \ldots & \ldots & \ldots & \ldots & \ldots & \ldots & \ldots & \ldots & \ldots \\ \ldots & \ldots & \ldots & \ldots & \ldots & \ldots & \ldots & \ldots & \ldots & \ldots & \ldots \\ \ldots & \ldots & \ldots & \ldots & \ldots & \ldots & \ldots & \ldots & \ldots & \ldots & \ldots\end{array}$

C
C
C
C
C
C

$\ldots$ . .

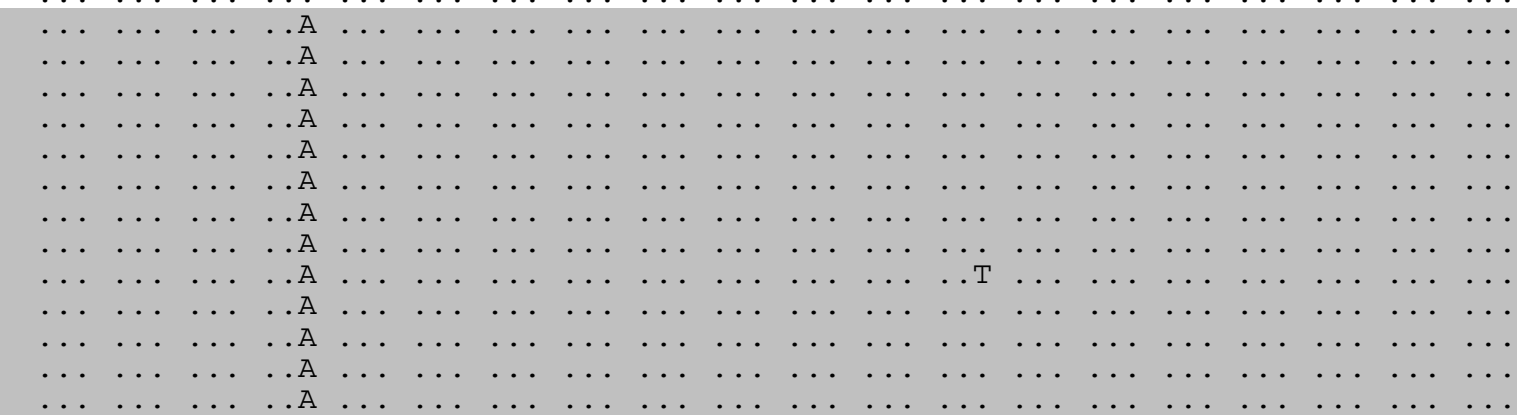

BK2bk2

A1a1

A2a1

A3a1

A4a2

CN1Cn1

CN1cn2

CN1cn3

CN1Cs1

CN2cn1

CN3cn 2

CN4Cn2

CS1cs1

CS1cs 2

CS2cs2

CS3cs 1

CS4cs2

M.clamit

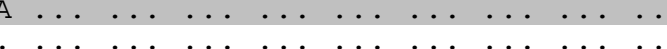

$\begin{array}{lllllllllllll}\ldots & \cdots & \ldots & \ldots & \ldots & \ldots & \ldots & \ldots & \ldots & \ldots & \ldots & \ldots & \ldots \\ \cdots & \cdots & \cdots & \ldots & \cdots & \cdots & \ldots & \cdots & \cdots & \ldots & \ldots & \ldots & \ldots\end{array}$

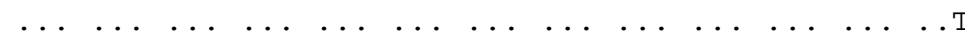

$\begin{array}{lllllllllllll}\ldots & \ldots & \ldots & \ldots & \ldots & \ldots & \ldots & \ldots & \ldots & \ldots & \ldots & \ldots & \ldots\end{array}$

$\begin{array}{lllllllllllll}\ldots & \ldots & \ldots & \ldots & \ldots & \ldots & \ldots & \ldots & \ldots & \ldots & \ldots & \ldots & \ldots T \\ \ldots & \ldots & \ldots & \ldots & \ldots & \ldots & \ldots & \ldots & \ldots & \ldots & \ldots & \ldots & \ldots\end{array}$

$\begin{array}{lllllllllllll}\ldots & \ldots & \ldots & \ldots & \ldots & \ldots & \ldots & \ldots & \ldots & \ldots & \ldots & \ldots & \ldots T \\ \ldots & \ldots & \ldots & \ldots & \ldots & \ldots & \ldots & \ldots & \ldots & \ldots & \ldots & \ldots & \ldots\end{array}$

(1,

$\begin{array}{lllllllllllll}\ldots & \ldots & \ldots & \ldots & \ldots & \ldots & \ldots & \ldots & \ldots & \ldots & \ldots & \ldots & \ldots\end{array}$

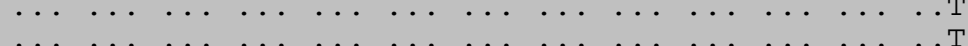

M. phaeop
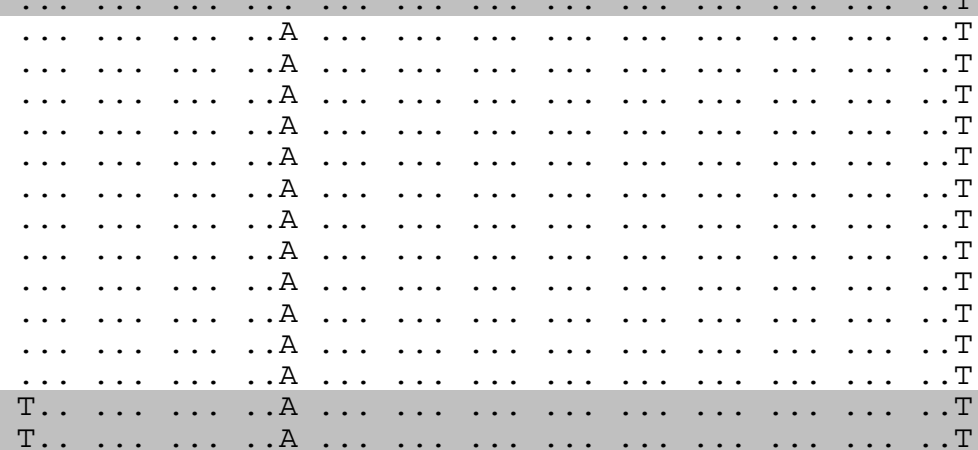
Appendix D: Halpotype sequences

COI

[660]

BSA1bsa1

ATT TCG TCA ATA gGA AGA ATT ATA TCC TTA ATC GGA ATT ATA TTT TTA TTA TTT ATT GTG

BSA1bsa3

BSA1bsa4

BSA1bsa5

BSA2bsa1

BSA3bsa1

BSA4bsa1

BSA5bsa1

BSA6bsa6

BSA7bsa11

BSA8bsa1

BSB1bsb1

BSB1bsb2

BSB1bsb3

BSB2bsb1

BSB3bsb1

BSB4bsb1

BSB5bsa7

BSC1bsa1

BSC2bsa1

BSC2bsa8

BSC3bsa9

BSC4bsa10

BSC5bsa1

BSC6bsa1

BW1bw1

BW1bw3

BW1bw5

BW2bw1

BW2bw3

BW3bw2

BW4bw1

BW5bw4

BW6bw1

BW7bw1

BW8bw6

BW9bw7

BW10bw2

$\begin{array}{llllllllllllllllllll}\ldots & \ldots & \ldots & \ldots & \ldots & \ldots & \ldots & \ldots & \ldots & \ldots & \ldots & \ldots & \ldots & \ldots & \ldots & \ldots & \ldots & \ldots & \ldots & \ldots \\ \ldots & \ldots & \ldots & \ldots & \ldots & \ldots & \ldots & \ldots & \ldots & \ldots & \ldots & \ldots & \ldots & \ldots & \ldots & \ldots & \ldots & \ldots & \ldots & \ldots\end{array}$

$\ldots \ldots \ldots$

$\cdots$

$\ldots$

.

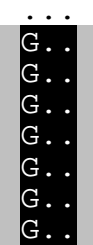

BM1bm1

BM1bm2

BM1bm3

BM1bm4

BM2bm1

BM2bm5

BM3bm1

BM4bm1

BM5bm2

BM6bm6

BN1bn1

BN1bn2

BN2bn1

BN3bn1

BK1bk1

BK2bk2

A1a1

A2a1

A3a1

A4a2

CN1cn1

CN1Cn2

CN1Cn3

CN1CS1

CN2Cn1

CN3Cn2

CN4Cn2

CS1cs1

CS1cs2

CS2cs2

CS3cs 1

CS4cs2

A

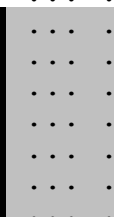

...

.
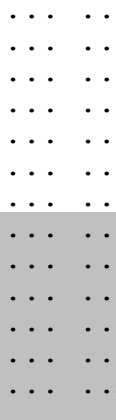
Appendix D: Halpotype sequences

COI

BSA1bsa1 BSA1bsa2

BSA1bsa3

BSA1bsa 4

BSA1bsa5

BSA2bsa1

BSA3bsa1

BSA4bsa1

BSA5bsa1

BSA6bsa6

BSA7bsa11

BSA8bsa1

BSB1bsb1

BSB1bsb2

BSB1bsb3

BSB2bsb1

BSB3bsb1

BSB4bsb1

BSB5bsa7

BSC1bsa1

BSC2bsa1

BSC2bsa8

BSC3bsa9

BSC4bsa10

BSC5bsa1

BSC6bsa1

BW1bw1

BW1bw3

BW1bw5

BW2bw1

BW2bw3

BW3bw2

BW4bw1

BW5bw4

BW6bw1

BW7bw1

BW8bw6

BW9bw7

BW10bw2

BM1bm1

BM1bm2

BM1bm3

BM1bm4

BM2bm1

BM2bm5

BM3bm1

BM4bm1

BM5bm2

BM6bm6

BN1bn1

BN1bn2

BN2bn1

BN3bn1

BK1bk1

BK2bk2

A1a1

A2a1

A3a1

A4a2

CN1Cn1

CN1Cn2

CN1cn3

CN1cs1

CN2Cn1

CN3cn2

CN4Cn2

CS1cs1

CS1cs 2

CS2cs2

CS3cs 1

CS4cs2

M. clamit

M. phaeop
TGg GAA AGA TTT ATT TCA ATG CGg TTA GTA TTA TAT TCT AAT AGA ATT CAg TCT TCT ATT

…

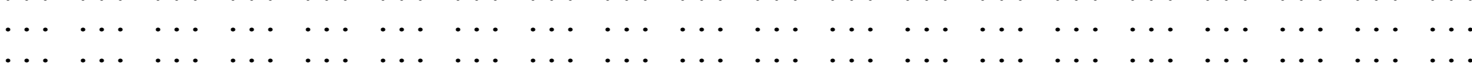

$\begin{array}{lllllllllllll}\ldots & \ldots & \ldots & \ldots & \ldots & \ldots & \ldots & \ldots & \ldots & \ldots & \ldots & \ldots & \ldots\end{array}$

$\cdots, \ldots$

A . 
Appendix D: Halpotype sequences

COI

tRNA ${ }^{\text {Asp }}$

[780]

BSA1bsa1

GAA TGA ATA CAG AAA TTT CCT CCA TCT GAA CAT TGG AAA AAT TAG TTT AAT AAA AAT ATA

BSA1bsa3

BSA1bsa4

BSA1bsa5

BSA2bsa1

BSA3bsa1

BSA4bsa1

BSA5bsa1

BSA6bsa6

BSA7bsa11

BSA8bsa1

BSB1bsb1

BSB1bsb2

BSB1bsb3

BSB2bsb1

BSB3bsb1

BSB4bsb1

BSB5bsa7

BSC1bsa1

BSC2bsa1

BSC2bsa8

BSC3bsa9

BSC4bsa10

BSC5bsa1

BSC6bsa1

BW1bw1

BW1bw3

BW1bw5

BW2bw1

BW2bw3

BW3bw2

BW4bw1

BW5bw4

BW6bw1

BW7bw1

BW8bw6

BW9bw7

BW10bw2

BM1bm1

BM1bm2

BM1bm3

BM1bm4

BM2bm1

BM2bm5

BM3bm1

BM4bm1

BM5bm2

BM6bm6

BN1bn1

BN1bn2

BN2bn1

$\begin{array}{llll}\ldots & \ldots & \ldots & \ldots \\ \ldots & \ldots & \ldots & \ldots \\ \ldots & \ldots & \ldots & \ldots \\ \ldots & \ldots & \ldots & \ldots \\ \ldots & \ldots & \ldots & \ldots \\ \ldots & \ldots & \ldots & \ldots T\end{array}$

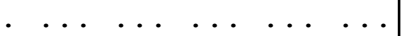

$\begin{array}{lllllllllll}\ldots & \ldots & \ldots & \ldots & \ldots & \ldots & \ldots & \ldots & \ldots & \ldots & \ldots \\ \ldots & \ldots & \ldots & \ldots & \ldots & \ldots & \ldots & \ldots & \ldots & \ldots & \ldots\end{array}$

$\begin{array}{lllllllllll}\ldots & \ldots & \ldots & \ldots & \ldots & \ldots & \ldots & \ldots & \ldots & \ldots & \ldots \\ \end{array}$

BN3bn1

BK1bk1

BK2bk2

A1a1

A2a1

A3a1

A4a2

CN1Cn1

CN1Cn2

CN1Cn3

CN1CS1

CN2Cn1

CN3Cn2

CN4Cn2

CS1cs1

CS1cs2

CS2cs2

CS3cs1

CS4cs2

M.clamit

M. phaeop

.
.
.
.
.
.
.
.
.
.
.
.
.

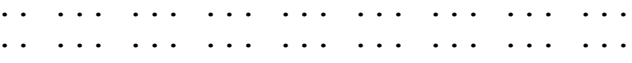




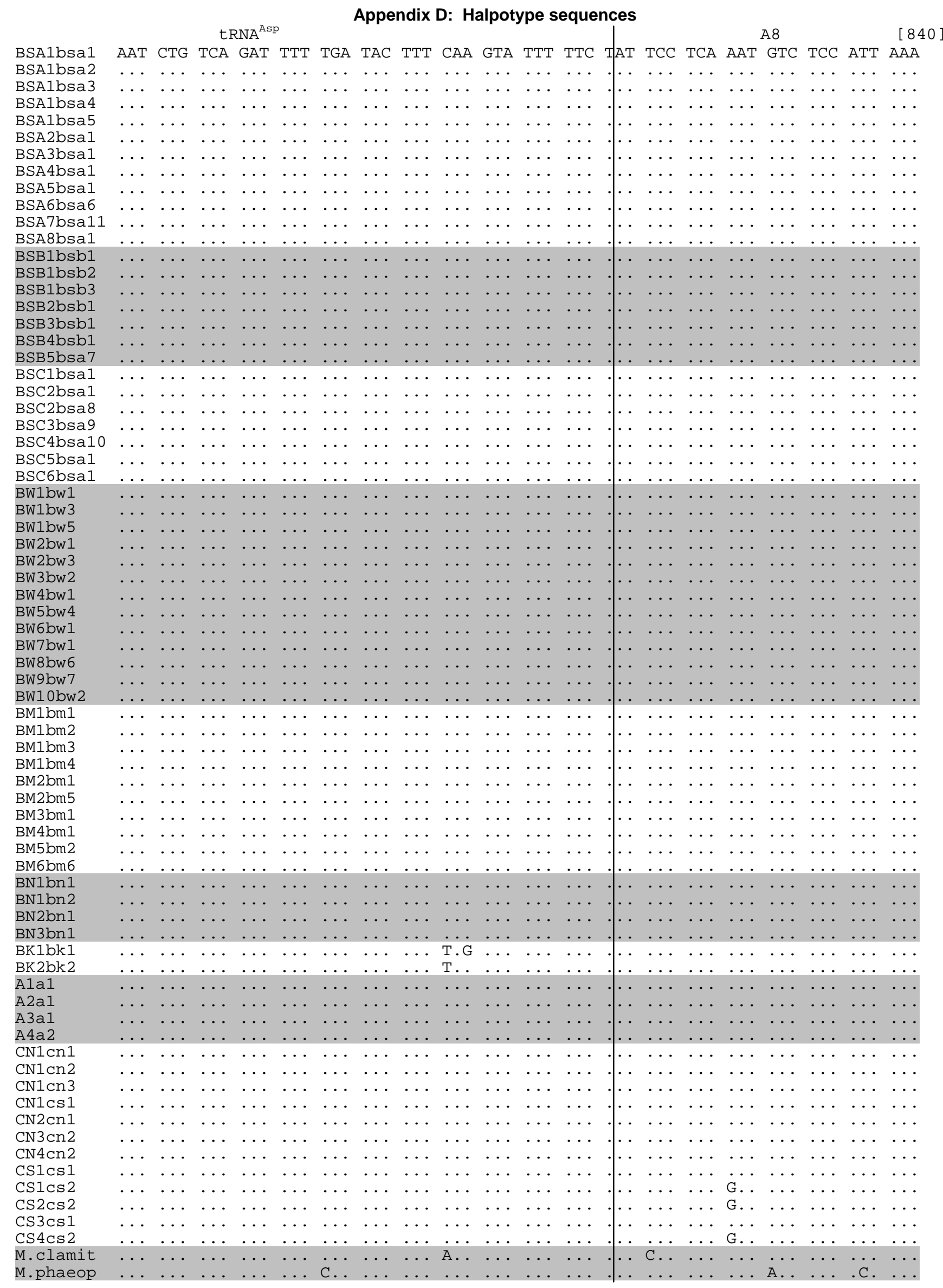


Appendix D: Halpotype sequences

A8

[900]

BSA1bsa1

TTG ATT ATT TTT ATT TAT TTA TTT TAT TAT AAT AAT TAT TTT TAT TAT TAT ATT TAT ATA

BSA1bsa2

BSA1bsa3

BSA1bsa4

BSA1bsa5

BSA2bsa1

BSA3bsa1

BSA4bsa1

BSA5bsa1

BSA6bsa6

BSA7bsa11

BSA8bsa1

BSB1bsb1

BSB1bsb2

BSB1bsb3

BSB2bsb1

BSB3bsb1

BSB4bsb1

BSB5bsa7

BSC1bsa1

BSC2bsa1

BSC2bsa8

BSC3bsa9

BSC4bsa10

BSC5bsa1

BSC6bsa1

BW1bw1

BW1bw3

BW1bw5

BW2bw1

BW2bw3

BW3bw2

BW4bw1

BW5bw4

BW6bw1

BW7bw1

BW8bw6

BW9bw7

BW10bw2

BM1bm1

BM1bm2

BM1bm3

BM1bm4

BM2bm1

BM2bm5

BM3bm1

BM4bm1

BM5bm2

BM6bm6

BN1bn1

BN1bn2

BN2bn1

BN3bn1

BK1bk1

BK2bk2

A1a1

A2a1

A3a1

A4a2

CN1cn1

CN1Cn2

CN1Cn3

CN1cs1

CN2Cn1

CN3Cn 2

CN4Cn2

CS1CS1

CS1cs 2

CS2cs2

CS3cs 1

CS4cs2

M. clamit

$\cdots \quad \cdots$

$\ldots \ldots$

$\cdots$

$\ldots$

....
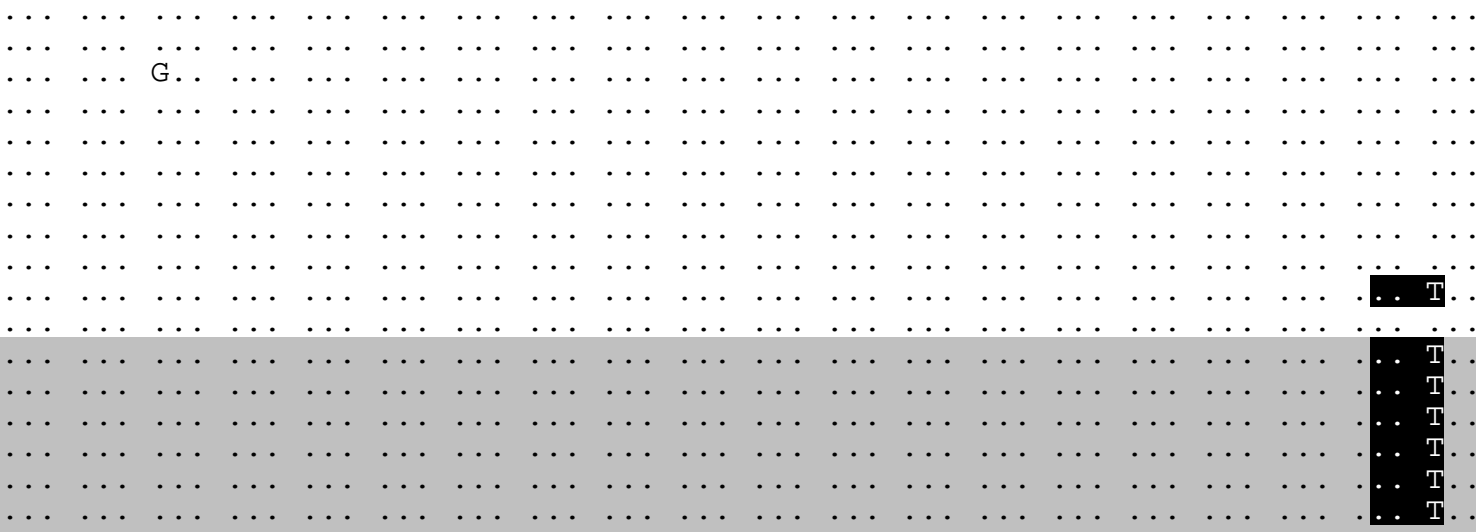

.

.
.
.
.

.

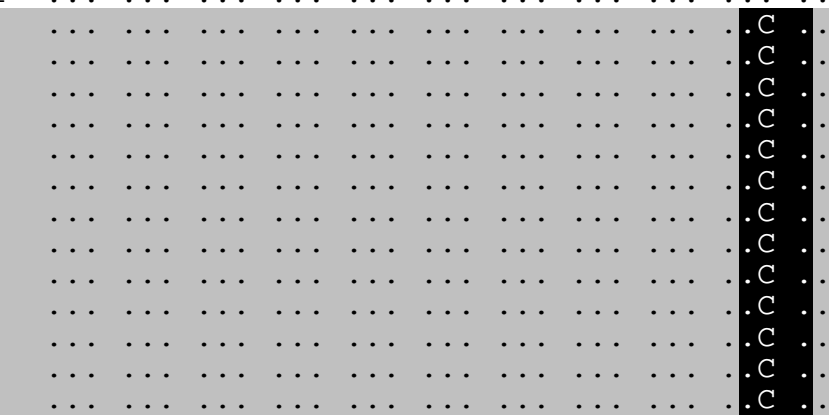

M. phaeop
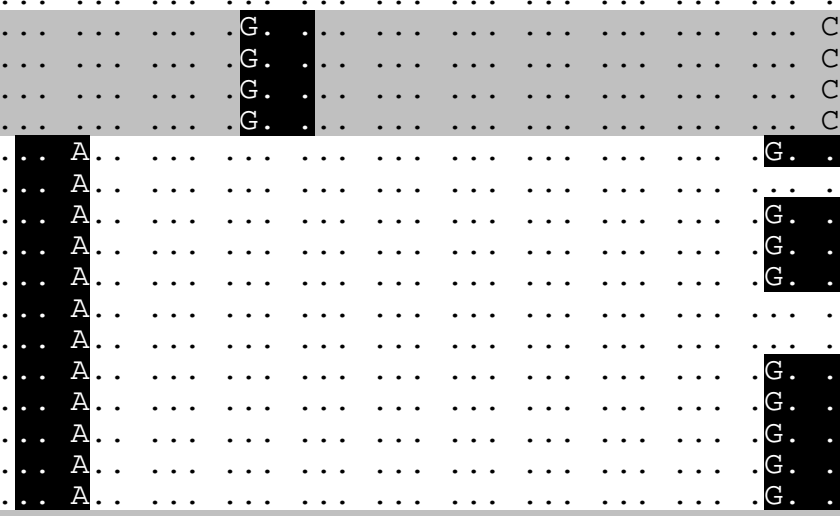

G

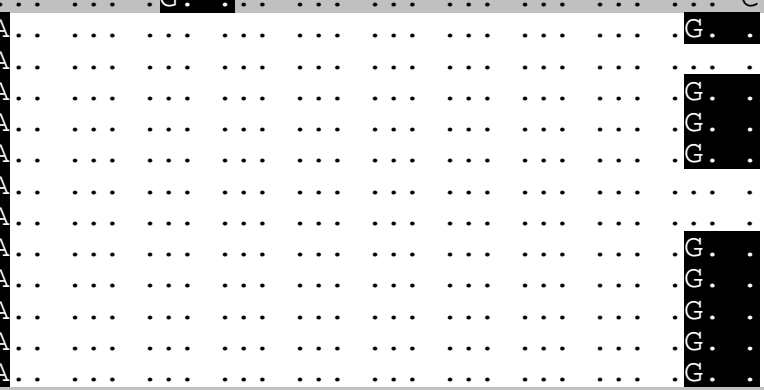


BSA1bsa1 TTT TGC TTG TTT ATT AAA ATC TAA TCT AAT AAC TAA TAA AAA GTT AAT AGA TAG AAT AAT

BSA1bsa2

BSA1bsa3

BSA1bsa 4

BSA1bsa5

BSA2bsa1

BSA3bsa1

BSA4bsa1

BSA5bsa1

BSA6bsa6

BSA7bsa11

BSA8bsa1

BSB1bsb1

BSB1bsb2

BSB1bsb3

BSB2bsb1

BSB3bsb1

BSB4bsb1

BSB5bsa 7

BSC1bsa1

BSC2bsa1

BSC2bsa8

BSC3bsa9

BSC4bsa10

BSC5bsa1

BSC6bsa1

BW1bw1

BW1bw3

BW1bw5

BW2bw1

BW2bw3

BW3bw2

BW4bw1

BW5bw4

BW6bw1

BW7bw1

BW8bw6

BW9bw7

BW10bw2

BM1bm1

BM1bm2

BM1bm3

BM1bm4

BM2bm1

BM2bm5

BM3bm1

BM4bm1

BM5bm2

BM6bm6

BN1bn1

BN1bn2

BN2bn1

BN3bn1

BK1bk1
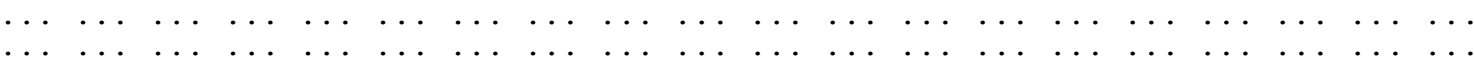

...

$\cdots$

$\ldots$

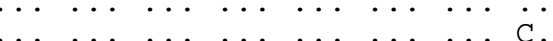

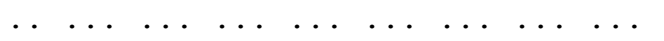

.

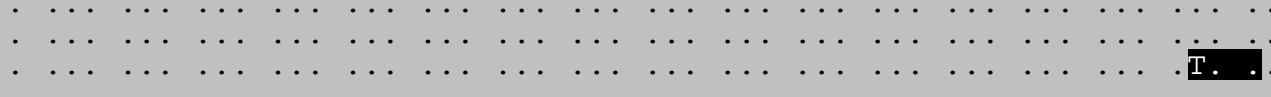

BK2bk2

A1a1

A2a1

A3a1

A4a2

CN1cn1

CN1cn2

CN1Cn3

CN1Cs1

$\mathrm{CN} 2 \mathrm{Cn} 1$

CN3cn2

CN4Cn2

CS1Cs1

CS1cs2

CS2cs2

CS3cs 1

cs4cs2

M. clamit
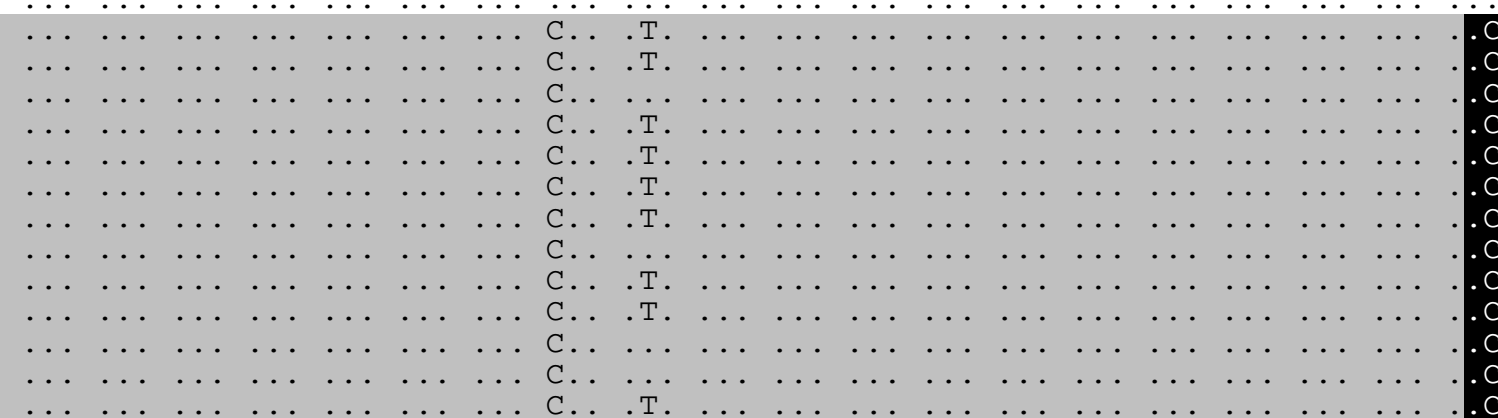

M. phaeop
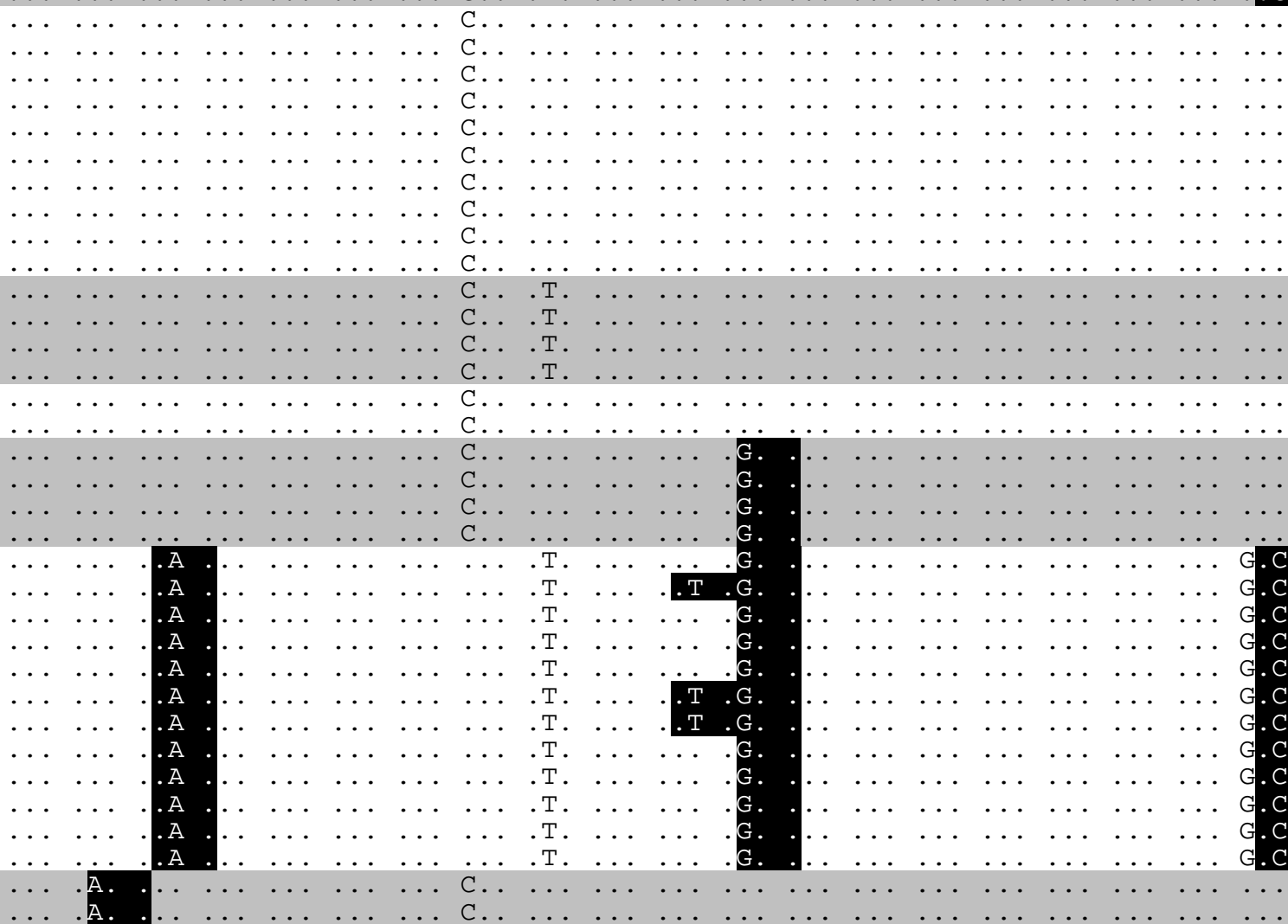
Appendix D: Halpotype sequences

A8 1 A8/A6 ل

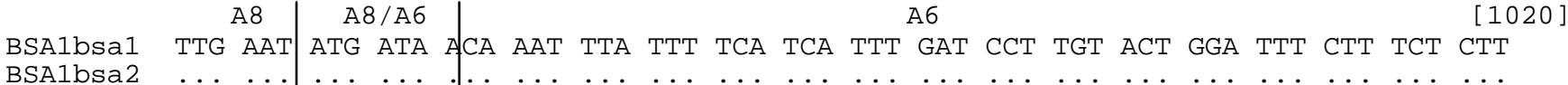

BSA1bsa3

BSA1bsa 4

BSA1bsa5

BSA2bsa1

BSA3bsa1

BSA4bsa1

BSA5bsa1

BSA6bsa 6

BSA7bsa11

BSA8bsa1

BSB1bsb1

BSB1bsb2

BSB1bsb3

BSB2bsb1

BSB3bsb1

BSB4bsb1

BSB5bsa7

BSC1bsa1

BSC2bsa1

BSC2bsa 8

BSC3bsa9

BSC4bsa10

BSC5bsa1

BSC6bsa1

BW1bw1

BW1bw3

BW1bw5

BW2bw1

BW2bw3

BW3bw2

BW4bw1

BW5bw4

BW6bw1

BW7bw1

BW8bw6

BW9bw7

BW10bw2

BM1bm1

BM1bm2

BM1bm3

BM1bm4

BM2bm1

BM2bm5

BM3bm1

BM4bm1

BM5bm2

BM6bm6

BN1bn1

BN1bn2

BN2bn1

BN3bn1

BK1bk1

BK2bk2

A1a1

A2a1

A3a1

A4a2

CN1cn1

CN1cn2

CN1cn3

CN1cs1

CN2cn1

CN3cn2

CN4Cn2

CS1cs1

CS1cs 2

CS2cs2

CS3cs 1

CS4cs2

M.clamit

M. phaeop 
Appendix D: Halpotype sequences

A6

BSA1bsa1

AAT TGA TTA AGA TCA ATA ATT TTG TTA TTA TTT TTA CCT ATT ACC TAT TGA TAT ATG CCA

BSA1bsa2

BSA1bsa3

BSA1bsa 4

BSA1bsa5

BSA2bsa1

BSA3bsa1

BSA4bsa1

BSA5bsa1

BSA6bsa6

BSA7bsa11

BSA8bsa1

BSB1bsb1

BSB1bsb2

BSB1bsb3

BSB2bsb1

BSB3bsb1

BSB4bsb1

BSB5bsa7

BSC1bsa1

BSC2bsa1

BSC2bsa8

BSC3bsa9

BSC4bsa10

BSC5bsa1

BSC6bsa1

BW1bw1

BW1bw3

BW1bw5

BW2bw1

BW2bw3

BW3bw2

BW4bw1

BW5bw4

BW6bw1

BW7bw1

BW8bw6

BW9bw7

BW10bw2

BM1bm1

BM1bm2

BM1bm3

BM1bm4

BM2bm1

BM2bm5

BM3bm1

BM4bm1

BM5bm2

BM6bm6

BN1bn1

BN1bn2

BN2bn1

BN3bn 1

...

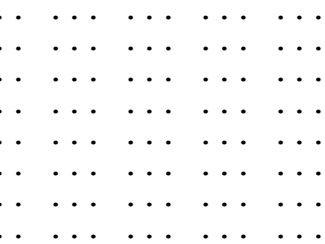

$\ldots \ldots, \ldots$

. 
Appendix D: Halpotype sequences

A6

[1140]

BSA1bsa1

AAC CGT TTT ATT ATT TTT TAT AAT AAA ATT TTA TTT TCA TTA AAT AAT GAA TTA AAT ATA

BSA1bsa2

BSA1bsa3

BSA1bsa 4

BSA1bsa5

BSA2bsa1

BSA3bsa1

BSA4bsa1

BSA5bsa1

BSA6bsa6

BSA7bsa11

BSA8bsa1

BSB1bsb1

BSB1bsb2

BSB1bsb3

BSB2bsb1

BSB3bsb1

BSB4bsb1

BSB5bsa 7

BSC1bsa1

BSC2bsa1

BSC2bsa8

BSC3bsa9

BSC4bsa10

BSC5bsa1

BSC6bsa1

BW1bw1

BW1bw3

BW1bw5

BW2bw1

BW2bw3

BW3bw2

BW4bw1

BW5bw4

BW6bw1

BW7bw1

BW8bw6

BW9bw7

BW10bw2

BM1bm1

BM1bm2

BM1bm3

BM1bm4

BM2bm1

BM2bm5

BM3bm1

BM4bm1

BM5bm2

BM6bm6

BN1bn1

BN1bn2

BN2bn1

BN3bn1

BK1bk1

BK2bk2

A1a1

A2a1

A3a1

A4a2

CN1cn1

CN1cn2

CN1cn3

CN1cs1

CN2cn1

CN3cn 2

CN4Cn2

CS1cs1

CS1cs2

CS2cs2

CS3cs 1

CS4cs2

M. clamit

M. phaeop

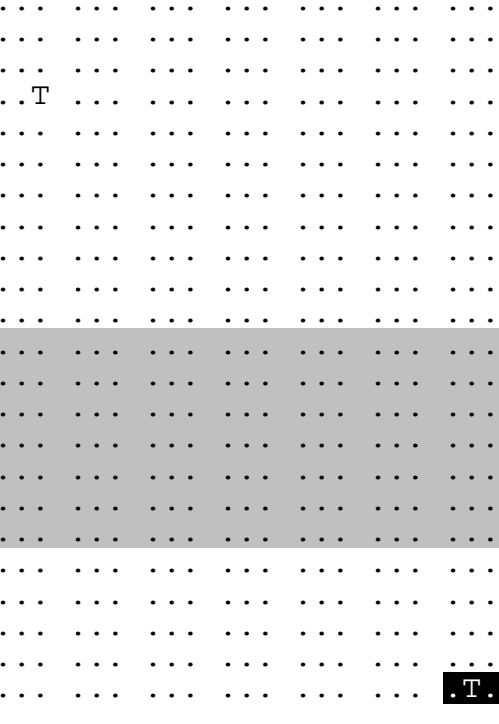

. T

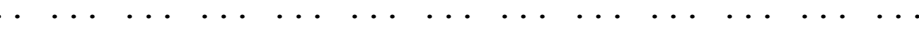


Appendix D: Halpotype sequences

A6

BSA1bsa1

TTA ATA AAT TAT AAA TCA TTA GgG AGA TCA TTA ATA TTT TTA TCA CTA TTT ACA TTT ATT

BSA1bsa2

BSA1bsa3

BSA1bsa 4

BSA1bsa5

BSA2bsa1

BSA3bsa1

BSA4bsa1

BSA5bsa1

BSA6bsa6

BSA7bsa11

BSA8bsa1

BSB1bsb1

BSB1bsb2

BSB1bsb3

BSB2bsb1

BSB3bsb1

BSB4bsb1

BSB5bsa7

BSC1bsa1

BSC2bsa1

BSC2bsa 8

BSC3bsa9

BSC4bsa10

BSC5bsa1

BSC6bsa1

BW1bw1

BW1bw3

BW1bw5

BW2bw1

BW2bw3

BW3bw2

BW4bw1

BW5bw4

BW6bw1

BW7bw1

BW8bw6

BW9bw7

BW10bw2

BM1bm1

BM1bm2

BM1bm3

BM1bm4

BM2bm1

BM2bm5

BM3bm1

BM4bm1

BM5bm2

BM6bm6

BN1bn1

BN1bn2

BN2bn1

BN3bn1

BK1bk1

BK2bk2

A1a1

A2a1

A3a1

A4a2

CN1cn1

CN1Cn2

CN1Cn3

CN1cs1

CN2Cn1

$\mathrm{CN} 3 \mathrm{Cn} 2$

CN4Cn2

CS1cs1

CS1cs2

CS2cs2

CS3cs 1

CS4cs2

M.clamit

$\cdots+\cdots$

$\ldots \ldots$

$\cdots$

$\cdots$

$\ldots \ldots$

.

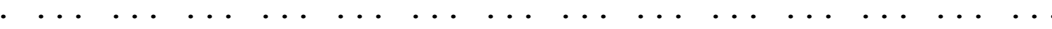

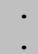

$\ldots$

$\cdots \cdots$

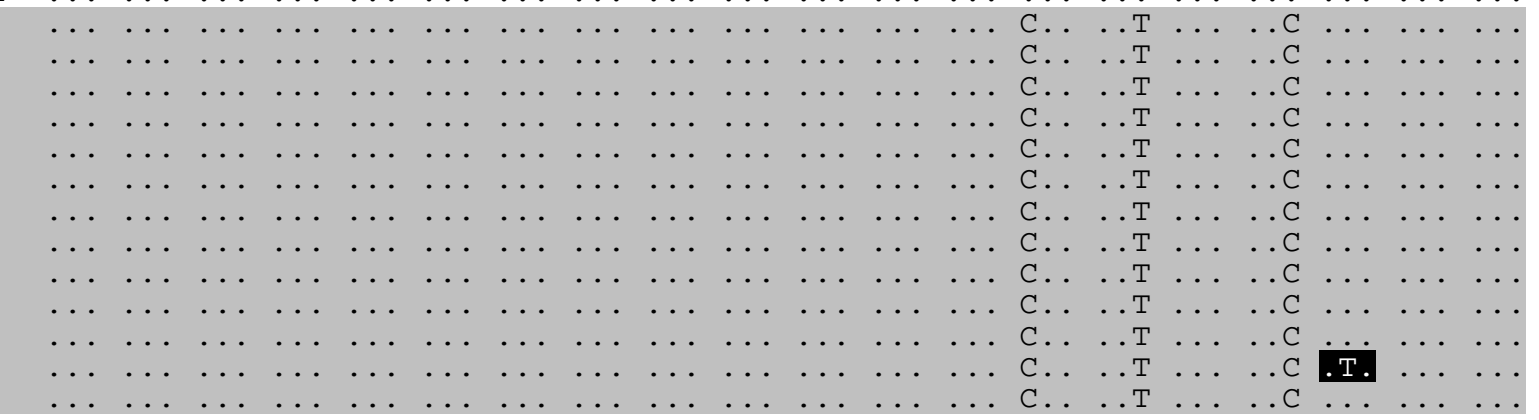

c.

$\begin{array}{lllllllllllll}\ldots & \ldots & \ldots & \ldots & \ldots & \ldots & \ldots & \ldots & \ldots & \ldots & \ldots & \ldots & \ldots\end{array}$

$\cdots, \ldots$.

$\ldots$

$\dot{C}$

С. . .

M. phaeop 
Appendix D: Halpotype sequences

A6

[1260]

BSA1bsa1

CTA TTA AAC AAT TTA TTA GGA TTA CTT CCT TAT ATT TTC ACT AGC TCT AGT CAC TTA GTA

BSA1bsa2

BSA1bsa3

BSA1bsa 4

BSA1bsa5

BSA2bsa1

BSA3bsa1

BSA4bsa1

BSA5bsa1

BSA6bsa6

BSA7bsa11

BSA8bsa1

BSB1bsb1

BSB1bsb2

BSB1bsb3

BSB2bsb1

BSB3bsb1

BSB4bsb1

BSB5bsa7

BSC1bsa1

BSC2bsa1

BSC2bsa8

BSC3bsa9

BSC4bsa10

BSC5bsa1

BSC6bsa1

BW1bw1

BW1bw5

BW2bw1

BW2bw3

BW3bw2

BW4bw1

BW5bw4

BW6bw1

BW7bw1

BW8bw6

BW9bw7

BW10bw2

BM1bm1

BM1bm2

BM1bm3

BM1bm4

BM2bm1

BM2bm5

BM3bm1

BM4bm1

BM5bm2

BM6bm6

BN1bn1

BN1bn2

BN2bn1

BN3bn1

BK1bk1

BK2bk2

A1a1

A2a1

A3a1

A4a2

CN1cn1

CN1Cn2

CN1Cn3

CN1CS1

CN2Cn1

$\mathrm{CN} 3 \mathrm{Cn} 2$

CN4Cn2

CS1cs1

CS1cs 2

CS2cs2

CS3cs1

CS4cs2

M.clamit

$\begin{array}{llll}\ldots & \ldots & \ldots & \ldots \\ \ldots & \ldots & \ldots & \ldots \\ \ldots & \ldots & \ldots & \ldots\end{array}$

$\ldots \ldots$

$\cdots$

$\ldots, \ldots$

$\ldots \ldots \ldots$

.

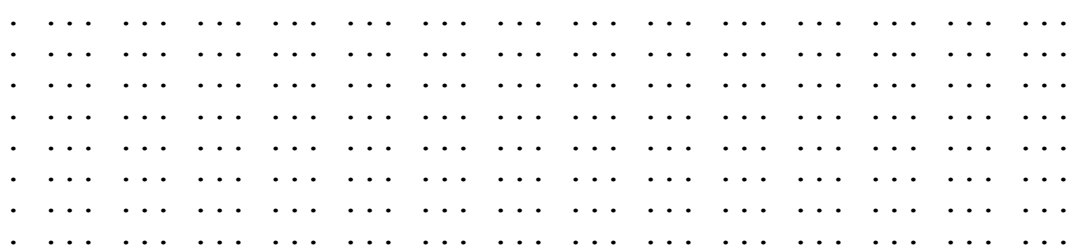

$\begin{array}{llll}\ldots & \ldots & \ldots & \ldots\end{array}$

\begin{tabular}{|c|c|c|c|c|c|c|c|c|c|c|c|c|c|c|c|c|}
\hline & & & . & & $\cdots$ & . & $\cdots$ & $\cdots$ & $\cdots$ & $\cdots$ & $\cdots$ & . & $\cdots$ & . & . & \\
\hline & 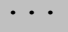 & . & $\cdots$ & . & & $\cdots$ & $\cdots$ & $\cdots$ & $\cdots$ & $\cdots$ & $\cdots$ & $\ldots$ & $\cdots$ & & & \\
\hline & $\cdots$ & $\cdots$ & $\cdots$ & $\cdots$ & $\cdots$ & $\cdots$ & $\cdots$ & $\cdots$ & $\cdots$ & $\cdots$ & $\cdots$ & $\cdots$ & $\cdots$ & $\cdots$ & $\cdots$ & \\
\hline & - & $\ldots$ & $\ldots$ & $\cdots$ & $\cdots$ & $\cdots$ & $\cdots$ & $\ldots$ & $\cdots$ & $\cdots$ & $\cdots$ & $\cdots$ & $\cdots$ & $\cdots$ & $\cdots$ & \\
\hline & $\cdots$ & $\cdots$ & $\cdots$ & $\cdots$ & $\cdots$ & $\cdots$ & $\cdots$ & $\cdots$ & $\cdots$ & $\cdots$ & $\cdots$ & $\cdots$ & $\cdots$ & $\cdots$ & $\cdots$ & \\
\hline
\end{tabular}

.

$\ldots \ldots$

..

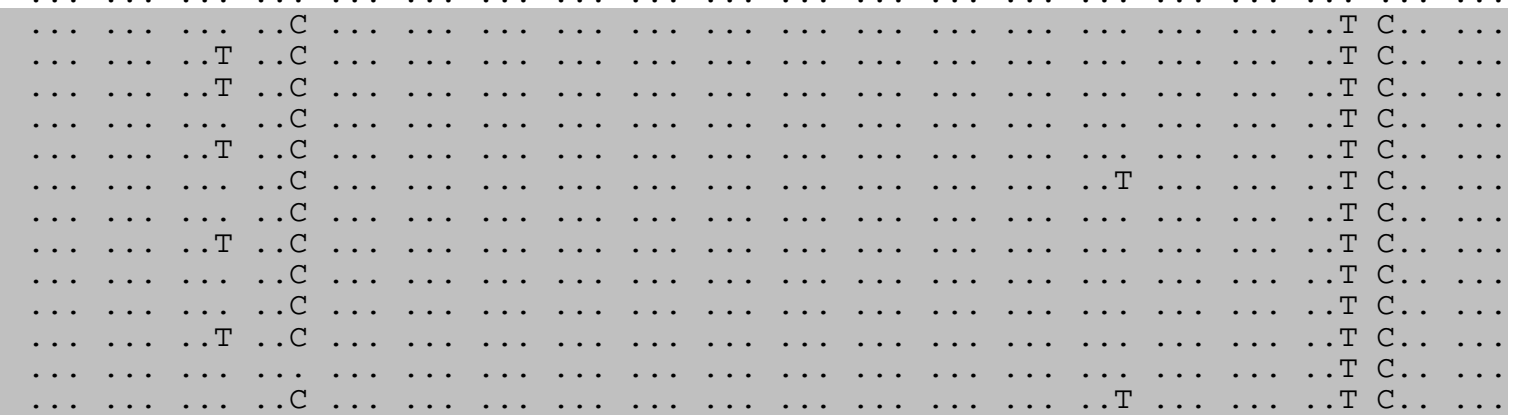

M. phaeop

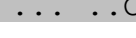

$T C$

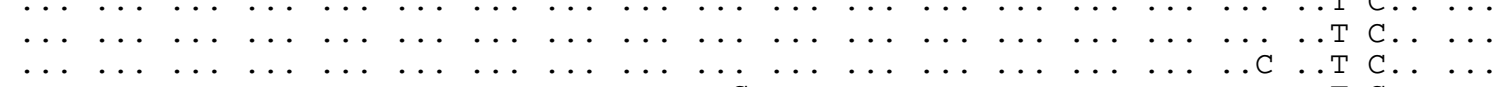

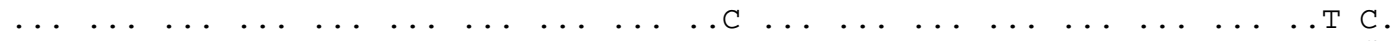

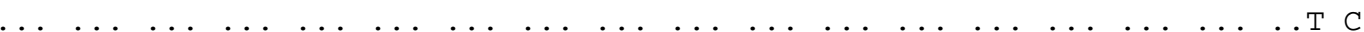

$\begin{array}{lllllllllllllllllllllllllll}\ldots & \ldots & \ldots & \ldots & \ldots & \ldots & \ldots & \ldots & \ldots & \ldots & \ldots & \ldots & \ldots & \ldots & \ldots & \ldots & \ldots & \ldots & \\ \end{array}$

$\ldots \begin{array}{lllllllllllllllllll}\ldots & \ldots & \ldots & \ldots & \ldots & \ldots & \ldots & \ldots & \ldots & \ldots & \ldots & \ldots & \ldots & \ldots & \ldots & \ldots & \ldots & \ldots & \\ \end{array}$

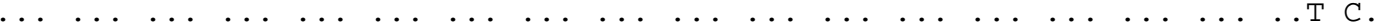

$\begin{array}{llllllllllllllllll}\ldots & \ldots & \ldots & \ldots & \ldots & \ldots & \ldots & \ldots & \ldots & \ldots & \ldots & \ldots & \ldots & \ldots & \ldots & \ldots & \ldots & \ldots \\ \mathrm{C}\end{array}$

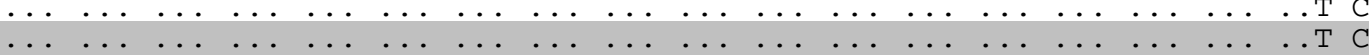

\begin{tabular}{llllllllllllllllll} 
& $\ldots$ & $\ldots$ & $\ldots$ & $\ldots$ & $\ldots$ & $\ldots$ & $\ldots$ & $\ldots$ & $\ldots$ & $\ldots$ & $\ldots$ & $\ldots$ & $\ldots$ & $\ldots$ & $\ldots$ & $\ldots$ & $\ldots$ \\
\hline
\end{tabular}

$\begin{array}{lllllllllllllllllllll}\ldots & \ldots & \ldots & \ldots & \ldots & \ldots & \ldots & \ldots & \ldots & \ldots & \ldots & \ldots & \ldots & \ldots & \ldots & \ldots & \ldots & \ldots & \\ \end{array}$

\begin{tabular}{llllllllllllllllll} 
& $\ldots$ & $\ldots$ & $\ldots$ & $\ldots$ & $\ldots$ & $\ldots$ & $\ldots$ & $\ldots$ & $\ldots$ & $\ldots$ & $\ldots$ & $\ldots$ & $\ldots$ & $\ldots$ & $\ldots$ & $\ldots$ & $\ldots$ \\
\hline
\end{tabular}

$\begin{array}{llllllllllllllllll}\ldots & \ldots & \ldots & \ldots & \ldots & \ldots & \ldots & \ldots & \ldots & \ldots & \ldots & \ldots & \ldots & \ldots & \ldots & \ldots & \ldots & \ldots\end{array}$

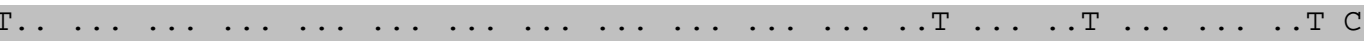

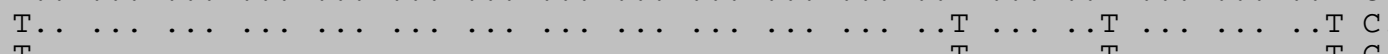

$\begin{array}{llllllllllllllllllll}\mathrm{T} \ldots & \ldots & \ldots & \ldots & \ldots & \ldots & \ldots & \ldots & \ldots & \ldots & \ldots & \ldots & \ldots & \ldots & \ldots \mathrm{T} & \ldots & \ldots & \ldots \mathrm{T} & \mathrm{C} \\ \mathrm{T} & \ldots & \ldots & & & \ldots\end{array}$

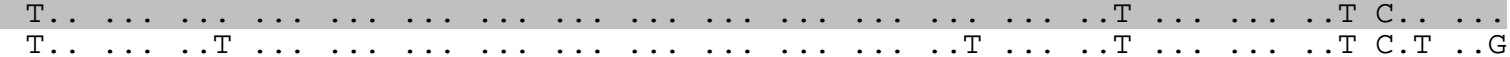

T.

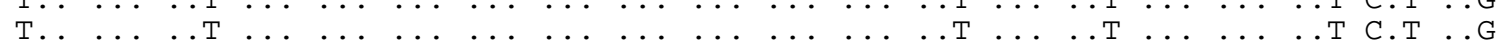

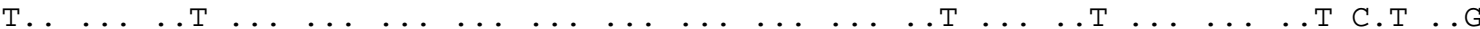

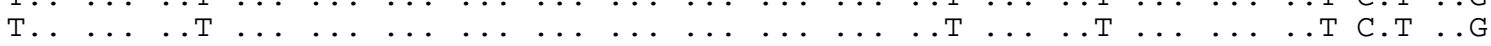

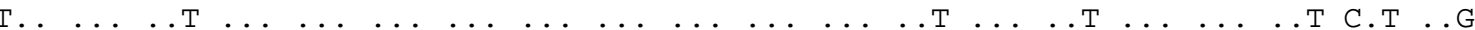

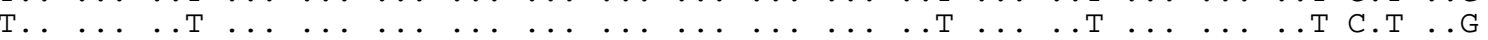

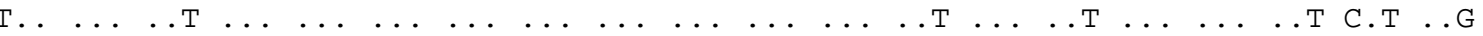

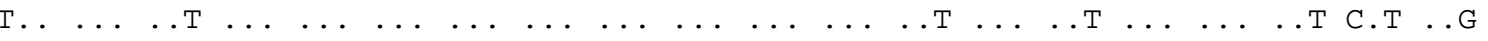

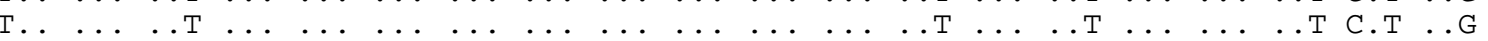

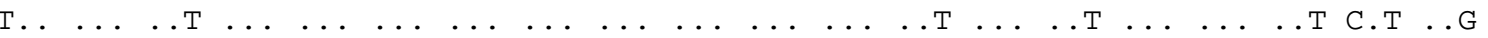

\begin{tabular}{llllllllllllllllllll} 
& $\ldots$ & $\ldots$ & $\ldots$ & $\ldots$ & $\ldots$ & $\ldots$ & $\ldots$ & $\ldots$ & $\ldots$ & $\ldots$ & $\ldots$ & $\ldots$ & $\ldots$ & $\ldots T$ & $\ldots$ & $\ldots T$ & $\ldots$ & $\ldots$ & $\ldots T$ \\
\hline
\end{tabular}

$\begin{array}{llllllllllllllllllll}\mathrm{T} \ldots & \ldots & \ldots & \ldots & \ldots & \ldots \mathrm{G} & \ldots & \ldots & \ldots & \ldots & \ldots & \ldots & \ldots & \ldots & \ldots \mathrm{T} & \ldots & \ldots & \ldots \mathrm{T} & \ldots & \ldots \mathrm{G} \\ \mathrm{T} \ldots & \ldots \mathrm{G} & \ldots & \ldots & \ldots & \ldots & \ldots & \ldots & \ldots & \ldots & \ldots & \ldots & \ldots \mathrm{T} & \ldots \mathrm{A} & \ldots \mathrm{T} & \ldots & \ldots & \ldots \mathrm{T} & \ldots & \ldots \mathrm{G}\end{array}$ 
Appendix D: Halpotype sequences

A6

[1320]

BSA1bsa1 TTT ACA ATA AGA TTA GCG TTA CCC TTG TGA TTA TCA TTT ATG CTT TAT GGA TTT ATT AAT

BSA1bsa2

BSA1bsa3

BSA1bsa 4

BSA1bsa5

BSA2bsa1

BSA3bsa1

BSA4bsa1

BSA5bsa1

BSA6bsa6

BSA7bsa11

BSA8bsa1

BSB1bsb1

BSB1bsb2

BSB1bsb3

BSB2bsb1

BSB3bsb1

BSB4bsb1

BSB5bsa7

BSC1bsa1

BSC2bsa1

BSC2bsa8

BSC3bsa9

BSC4bsa10

BSC5bsa1

BSC6bsa1

BW1bw1

BW1bw5

BW2bw1

BW2bw3

BW3bw2

BW4bw1

BW5bw4

BW6bw1

BW7bw1

BW8bw6

BW9bw7

BW10bw2

BM1bm1

BM1bm2

BM1bm3

BM1bm4

BM2bm1

BM2bm5

BM3bm1

BM4bm1

BM5bm2

BM6bm6

BN1bn1

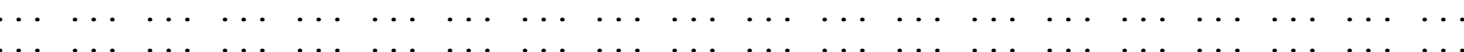

$\cdots+\cdots$

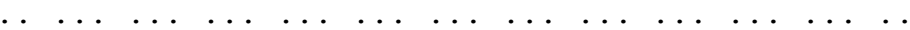

$\cdots, \cdots$

$\cdots+\cdots$

$\ldots \ldots+\ldots, \ldots$

$\begin{array}{llllll}\ldots & \ldots & \ldots & \ldots & \ldots & \ldots \\ \ldots & \ldots & \ldots & \ldots & \ldots & \ldots\end{array}$

$\begin{array}{lllllll}\ldots & \ldots & \ldots & \ldots & \ldots & \ldots & \ldots \\ & \ldots & \ldots & \ldots\end{array}$

$\ldots \begin{array}{lllllll}\ldots & \ldots & \ldots & \ldots & \ldots & \ldots & \ldots\end{array}$

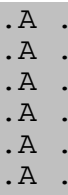

G. .

BN1bn2

BN2bn1

BN3bn1

BK1bk1

BK2bk2

A1a1

A2a1

A3a1

A4a2

CN1Cn1

CN1Cn2

CN1cs1

CN1Cn3

CN2Cn1

CN3Cn2

CN4Cn2

CS1cs1

CS1cs2

CS2cs2

CS3cs 1

CS4cs2

M. clamit

M. phaeop

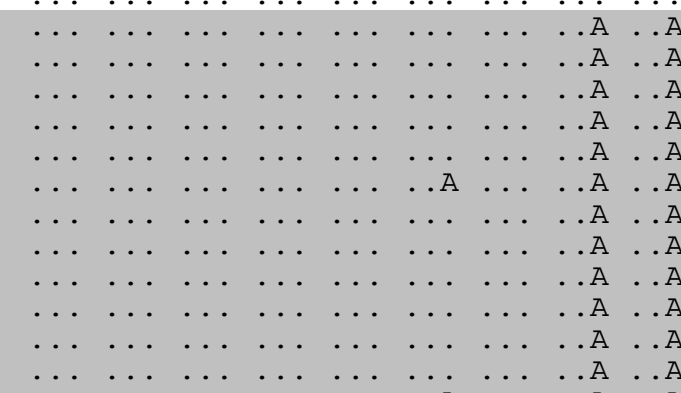


Appendix D: Halpotype sequences

A6

BSA1bsa1

AAT GTT AAT CAT ATA TTT TGT CAT ATA GTT CCA TCA GGA ACT CCT AAT ATT TTA ATA CCT

BSA1bsa2

BSA1bsa3

BSA1bsa 4

BSA1bsa5

BSA2bsa1

BSA3bsa1

BSA4bsa1

BSA5bsa1

BSA6bsa6

BSA7bsa11

BSA8bsa1

BSB1bsb1

BSB1bsb2

BSB1bsb3

BSB2bsb1

BSB3bsb1

BSB4bsb1

BSB5bsa7

BSC1bsa1

BSC2bsa1

BSC2bsa 8

BSC3bsa9

BSC4bsa10

BSC5bsa1

BSC6bsa1

BW1bw1

BW1bw3

BW1bw5

BW2bw1

BW2bw3

BW3bw2

BW4bw1

BW5bw4

BW6bw1

BW7bw1

BW8bw6

BW9bw7

BW10bw2

BM1bm1

BM1bm2

BM1bm3

BM1bm4

BM2bm1

BM2bm5

BM3bm1

BM4bm1

BM5bm2

BM6bm6

BN1bn1

BN1bn2

BN2bn1

BN3bn1

BK1bk1

BK2bk2

A1a1

A2a1

A3a1

A4a2 $\begin{array}{llllllllllllllllllll}\ldots & \ldots & \ldots & \ldots & \ldots & \ldots & \ldots & \ldots & \ldots & \ldots & \ldots & \ldots & \ldots & \ldots & \ldots & \ldots & \ldots & \ldots & \ldots & \ldots \\ \ldots & \ldots & \ldots & \ldots & \ldots & \ldots & \ldots & \ldots & \ldots & \ldots & \ldots & \ldots & \ldots & \ldots & \ldots & \ldots & \ldots & \ldots & \ldots & \ldots\end{array}$ $\begin{array}{llllllllllllllllllll}\ldots & \ldots & \ldots & \ldots & \ldots & \ldots & \ldots & \ldots & \ldots & \ldots & \ldots & \ldots & \ldots & \ldots & \ldots & \ldots & \ldots & \ldots & \ldots & \ldots\end{array}$

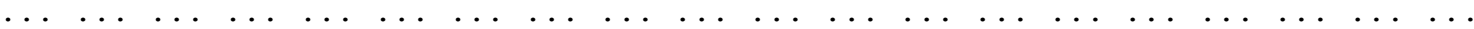

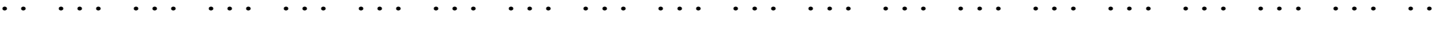

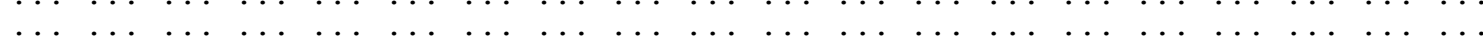
$\begin{array}{lllllllllllllllllll}\ldots & \ldots & \ldots & \ldots & \ldots & \ldots & \ldots & \ldots & \ldots & \ldots & \ldots & \ldots & \ldots & \ldots & \ldots & \ldots & \ldots & \ldots & \ldots \\ \ldots\end{array}$ .

$\ldots$ .

$\cdots \cdot \cdots$ (1)

(1)

CN1cn1

CN1cn2

CN1cn3

CN1Cs1

CN2Cn1

CN3cn2

CN4Cn2

CS1cs1

CS1cs 2

CS2cs2

CS3cs 1

CS4cs2

M.clamit M. phaeop

$\begin{array}{lllllllllll}\ldots & \ldots & \ldots & \ldots & \ldots & \ldots & \ldots & \ldots & \ldots & \ldots & \ldots \mathrm{G} \\ \ldots & \ldots & \ldots & \ldots & \ldots & \ldots & \ldots & \ldots & \ldots & \ldots & \ldots G \\ & \ldots & \ldots & \ldots & \ldots & \ldots & \ldots & \ldots & \ldots & \ldots & \ldots G\end{array}$

$\mathrm{G}$

$\begin{array}{lll}\ldots & \ldots & \ldots \\ \ldots & \ldots & \ldots \\ \ldots & \ldots & \ldots C \\ \ldots & \ldots & \ldots C\end{array}$

$\begin{array}{lllllllllll}\ldots & \ldots & \ldots & \ldots & \ldots & \ldots & \ldots & \ldots & \ldots & \ldots & \ldots \\ \ldots & \ldots & \ldots & \ldots & \ldots & \ldots & \ldots & \ldots & \ldots & \ldots & \ldots\end{array}$

,

$\begin{array}{llllllllllllllllllll} & \ldots & \ldots & \ldots & \ldots & \ldots & \ldots & \ldots & \ldots & \ldots & \ldots & \ldots & \ldots & \ldots & \ldots & \ldots & \ldots & \ldots & \ldots & \ldots\end{array}$

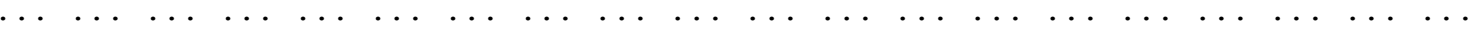

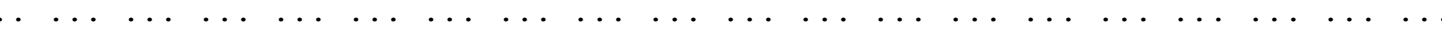

$\begin{array}{llllllllllllllllllll}\ldots & \ldots & \ldots & \ldots & \ldots & \ldots & \ldots & \ldots & \ldots & \ldots & \ldots & \ldots & \ldots & \ldots & \ldots & \ldots & \ldots & \ldots & \ldots & \ldots\end{array}$

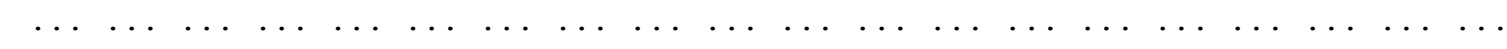

$\begin{array}{lllllllllllllllllll}\ldots & \ldots & \ldots & \ldots & \ldots & \ldots & \ldots & \ldots & \ldots & \ldots & \ldots & \ldots & \ldots & \ldots & \ldots & \ldots & \ldots & \ldots & \ldots \\ \ldots & \ldots & \ldots & \ldots & \ldots & \ldots & \ldots & \ldots & \ldots & \ldots & \ldots & \ldots & \ldots & \ldots & \ldots & \ldots & \ldots & \ldots\end{array}$ 
Appendix D: Halpotype sequences

A6

[1440]

BSA1bsa1 TTT ATA GTT ATT ATT GAA AGA ATT AGA AAT TTA ATT CGA CCG GGA TCA TTA GCT GTT CGT

BSA1bsa2

BSA1bsa3

BSA1bsa 4

BSA1bsa5

BSA2bsa1

BSA3bsa1

BSA4bsa1

BSA5bsa1

BSA6bsa6

BSA7bsa11

BSA8bsa1

BSB1bsb1

BSB1bsb2

BSB1bsb3

BSB2bsb1

BSB3bsb1

BSB4bsb1

BSB5bsa7

BSC1bsa1

BSC2bsa1

BSC2bsa8

BSC3bsa9

BSC4bsa10

BSC5bsa1

BSC6bsa1

BW1bw1

BW1bw3

BW1bw5

BW2bw1

BW2bw3

BW3bw2

BW4bw1

BW5bw4

BW6bw1

BW7bw1

BW8bw6

BW9bw7

BW10bw2

BM1bm1

BM1bm2

BM1bm3

BM1bm4

BM2bm1

BM2bm5

BM3bm1

BM4bm1

BM5bm2

BM6bm6

BN1bn1

BN1bn2

BN2bn1

BN3bn1

BK1bk1

BK2bk2

A1a1

A2a1

A3a1

A4a2

CN1cn1

CN1cn2

CN1cn3

CN1cs1

CN2cn1

CN3cn 2

CN4Cn2

CS1cs1

CS1cs2

CS2cs 2

CS3cs 1

CS4cs2

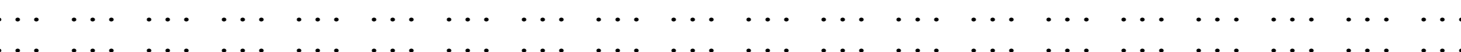

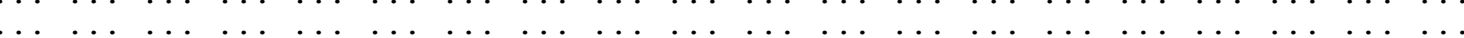

$\begin{array}{llllllllllllllllllllllllll}\ldots & \ldots & \ldots & \ldots & \ldots & \ldots & \ldots & \ldots & \ldots & \ldots & \ldots & \ldots & \ldots & \ldots & \ldots & \ldots & \ldots & \ldots & \ldots & \ldots\end{array}$

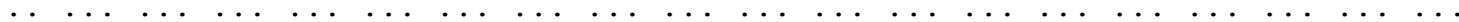

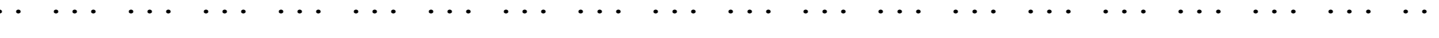

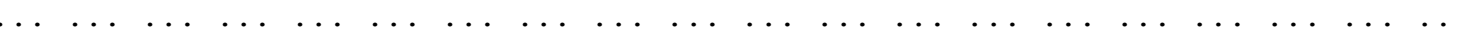

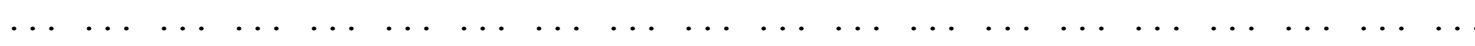
$\begin{array}{llllllllllllllll}\ldots & \ldots & \ldots & \ldots & \ldots & \ldots & \ldots & \ldots & \ldots & \ldots & \ldots & \ldots & \ldots & \ldots & \ldots & \ldots\end{array}$ $\begin{array}{llllllllllllll}\ldots & \ldots & \ldots & \ldots & \ldots & \ldots & \ldots & \ldots & \ldots & \ldots & \ldots & \ldots & \ldots & \ldots \\ \ldots & \ldots & \ldots & \ldots & \ldots & \ldots\end{array}$

$\begin{array}{lllllllllllll}\ldots & \ldots & \ldots & \ldots & \ldots & \ldots & \ldots & \ldots & \ldots & \ldots & \ldots & \ldots & \ldots \\ \ldots & \ldots & \ldots & \ldots & \ldots & \ldots & \ldots & \ldots & \ldots & \ldots\end{array}$

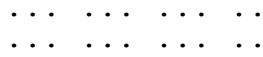

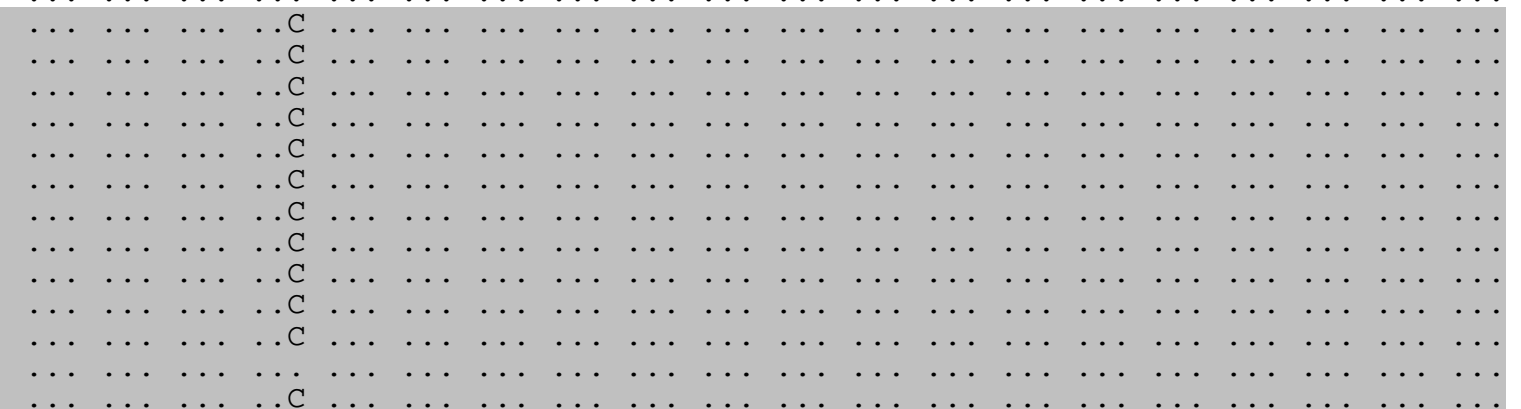

M.clamit

M. phaeop
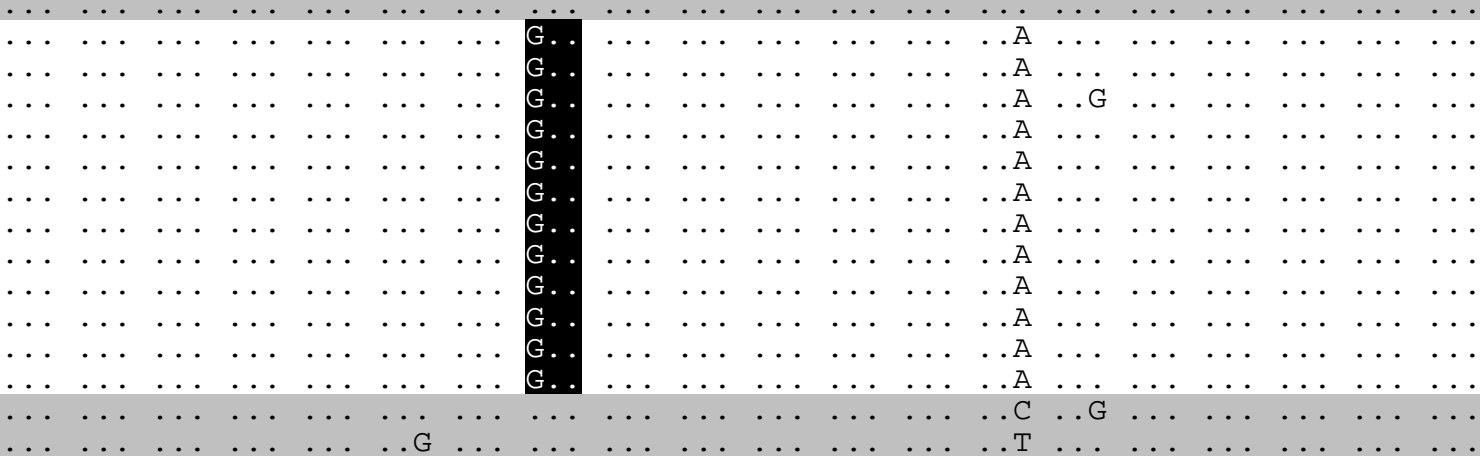
Appendix D: Halpotype sequences

A6

BSA1bsa1

TTA ACA GCT AAC ATA ATT GCT GGC CAT CTT TTA ATA ACT CTG CTT GGT AAT TTA CCT ATA

BSA1bsa2

BSA1bsa3

BSA1bsa 4

BSA1bsa5

BSA2bsa1

BSA3bsa1

BSA4bsa1

BSA5bsa1

BSA6bsa6

BSA7bsa11

BSA8bsa1

BSB1bsb1

BSB1bsb2

BSB1bsb3

BSB2bsb1

BSB3bsb1

BSB4bsb1

BSB5bsa7

BSC1bsa1

BSC2bsa1

BSC2bsa 8

BSC3bsa9

BSC4bsa10

BSC5bsa1

BSC6bsa1

BW1bw1

BW1bw5

BW2bw1

BW2bw3

BW3bw2

BW4bw1

BW5bw4

BW6bw1

BW7bw1

BW8bw6

BW9bw7

BW10bw2

BM1bm1

BM1bm2

BM1bm3

BM1bm4

BM2bm1

BM2bm5

BM3bm1

BM4bm1

BM5bm2

BM6bm6

BN1bn1

BN1bn2

BN2bn1

BN3bn1

BK1bk1

$\begin{array}{lllllllllllllllllllll}\ldots & \ldots & \ldots & \ldots & \ldots & \ldots & \ldots & \ldots & \ldots & \ldots & \ldots & \ldots & \ldots & \ldots A & \ldots & \ldots & \ldots & \ldots & \ldots & \ldots \\ \ldots & \ldots & \ldots & \ldots & \ldots & \ldots & \ldots & \ldots & \ldots & \ldots & \ldots & \ldots & \ldots & \ldots & \ldots & \ldots & \ldots & \ldots & \ldots & \ldots\end{array}$

$\ldots \ldots \ldots$

$\begin{array}{llllllllllllll} & \ldots & \ldots & \ldots & \ldots & \ldots & \ldots & \ldots & \ldots & \ldots & \ldots & \ldots & \ldots\end{array}$

$\cdots, \ldots$

$\cdots$

$\cdots+\ldots, \ldots$

. 


\begin{tabular}{|c|c|c|c|c|c|c|c|}
\hline \multirow[b]{2}{*}{ BSA1bsa1 } & \multicolumn{6}{|c|}{ A6 } & $\begin{array}{l}\text { Арр } \\
{[15}\end{array}$ \\
\hline & AAT & TAT & GAA & TAT & TAT & TCA & $\mathrm{GG}$ \\
\hline BSA1bsa2 & $\ldots$ & $\ldots$ & $\ldots$ & $\ldots$ & $\ldots$ & & . \\
\hline BSA1bsa3 & $\ldots$ & $\ldots$ & $\ldots$ & $\ldots$ & $\ldots$ & & . \\
\hline BSA1bsa4 & $\ldots$ & $\ldots$ & $\ldots$ & $\ldots$ & $\ldots$ & $\ldots$ & . \\
\hline BSA1bsa5 & $\ldots$ & $\ldots$ & $\ldots$ & $\ldots$ & $\ldots$ & $\ldots$ & . \\
\hline BSA2bsa1 & $\ldots$ & $\ldots$ & $\ldots$ & $\ldots$ & $\ldots$ & $\ldots$ & . \\
\hline BSA3bsa1 & $\ldots$ & $\ldots$ & $\ldots$ & $\ldots$ & $\ldots$ & $\ldots$ & . \\
\hline BSA4bsa1 & $\ldots$ & $\ldots$ & $\ldots$ & $\ldots$ & $\ldots$ & $\ldots$ & . \\
\hline BSA5bsa1 & $\ldots$ & $\ldots$ & $\ldots$ & $\ldots$ & $\ldots$ & $\ldots$ & . \\
\hline BSA6bsa6 & $\ldots$ & $\ldots$ & $\ldots$ & $\ldots$ & $\ldots$ & $\ldots$ & . \\
\hline BSA7bsa11 & $\ldots$ & $\ldots$ & $\ldots$ & $\ldots$ & $\ldots$ & $\ldots$ & . \\
\hline BSA8bsa1 & $\ldots$ & $\ldots$ & $\ldots$ & $\ldots$ & $\ldots$ & $\ldots$ & . \\
\hline BSB1bsb1 & $\ldots$ & $\ldots$ & $\ldots$ & $\ldots$ & $\ldots$ & $\ldots$ & . \\
\hline BSB1bsb2 & $\ldots$ & $\ldots$ & $\ldots$ & $\ldots$ & $\ldots$ & $\ldots$ & . \\
\hline BSB1bsb3 & $\ldots$ & $\ldots$ & $\ldots$ & $\ldots$ & $\ldots$ & $\ldots$ & . \\
\hline BSB2bsb1 & $\ldots$ & $\ldots$ & $\ldots$ & $\ldots$ & $\ldots$ & $\ldots$ & . \\
\hline BSB3bsb1 & $\ldots$ & $\ldots$ & $\ldots$ & $\ldots$ & $\ldots$ & $\ldots$ & . \\
\hline BSB4bsb1 & $\ldots$ & $\ldots$ & $\ldots$ & $\ldots$ & $\ldots$ & $\ldots$ & . \\
\hline BSB5bsa7 & $\ldots$ & $\ldots$ & $\ldots$ & $\ldots$ & $\ldots$ & $\ldots$ & . \\
\hline BSC1bsa1 & . & $\ldots$ & $\ldots$ & $\ldots$ & $\ldots$ & $\ldots$ & . \\
\hline BSC2bsa1 & $\ldots$ & $\ldots$ & $\ldots$ & $\ldots$ & $\ldots$ & $\ldots$ & . \\
\hline BSC2bsa 8 & $\ldots$ & $\ldots$ & $\ldots$ & $\ldots$ & $\ldots$ & $\ldots$ & . \\
\hline BSC3bsa9 & $\ldots$ & $\ldots$ & $\ldots$ & $\ldots$ & $\ldots$ & $\ldots$ & . \\
\hline BSC4bsa10 & $\ldots$ & $\ldots$ & $\ldots$ & $\ldots$ & $\ldots$ & $\ldots$ & . \\
\hline BSC5bsa1 & $\ldots$ & $\ldots$ & $\ldots$ & $\ldots$ & $\ldots$ & $\ldots$ & . \\
\hline BSC6bsa1 & $\ldots$ & $\ldots$ & $\ldots$ & $\ldots$ & $\ldots$ & $\ldots$ & . \\
\hline BW1bw1 & $\ldots$ & $\ldots$ &. $\mathrm{C}$. & $\ldots$ & $\ldots$ & $\ldots$ & . \\
\hline BW1bw3 & $\ldots$ & $\ldots$ & C. & $\ldots$ & $\ldots$ & $\ldots$ & . \\
\hline BW1bw5 & $\ldots$ & $\ldots$ & . C. & $\ldots$ & $\ldots$ & $\ldots$ & . \\
\hline BW2bw1 & $\ldots$ & $\ldots$ & . C. & $\ldots$ & $\ldots$ & $\ldots$ & . \\
\hline BW2bw3 & $\ldots$ & $\ldots$ & . C. & $\ldots$ & $\ldots$ & $\ldots$ & . \\
\hline BW3bw2 & $\ldots$ & $\ldots$ & . C. & $\ldots$ & $\ldots$ & $\ldots$ & . \\
\hline BW4bw1 & $\ldots$ & $\ldots$ & . C. & $\ldots$ & $\ldots$ & $\ldots$ & . \\
\hline BW5bw4 & $\ldots$ & $\ldots$ & . C. & $\ldots$ & $\ldots$ & $\ldots$ & . \\
\hline BW6bw1 & $\ldots$ & $\ldots$ & . C. & $\ldots$ & $\ldots$ & $\ldots$ & . \\
\hline BW7bw1 & $\ldots$ & $\ldots$ & . C. & $\ldots$ & $\ldots$ & $\ldots$ & . \\
\hline Bw8bw6 & $\ldots$ & $\ldots$ & . C. & $\ldots$ & $\ldots$ & $\ldots$ & . \\
\hline BW9bw7 & $\ldots$ & $\ldots$ & $\ldots$ & $\ldots$ & $\ldots$ & $\ldots$ & . \\
\hline BW10bw2 & $\ldots$ & $\ldots$ & . C. & $\ldots$ & $\ldots$ & $\ldots$ & . \\
\hline BM1bm1 & $\ldots$ & $\ldots$ & $\ldots$ & $\ldots$ & $\ldots$ & $\ldots$ & . \\
\hline BM1bm2 & $\ldots$ & $\ldots$ & $\ldots$ & $\ldots$ & $\ldots$ & $\ldots$ & . \\
\hline BM1bm3 & $\ldots$ & $\ldots$ & $\ldots$ & $\ldots$ & $\ldots$ & $\ldots$ & . \\
\hline BM1bm4 & $\ldots$ & $\ldots$ & $\ldots$ & $\ldots$ & $\ldots$ & $\ldots$ & . \\
\hline BM2bm1 & $\ldots$ & $\ldots$ & $\ldots$ & $\ldots$ & $\ldots$ & $\ldots$ & . \\
\hline BM2bm5 & $\ldots$ & $\ldots$ & $\ldots$ & $\ldots$ & $\ldots$ & $\ldots$ & . \\
\hline BM3bm1 & $\ldots$ & $\ldots$ & . & $\cdots$ & $\ldots$ & $\ldots$ & . \\
\hline BM4bm1 & $\ldots$ & $\ldots$ & $\ldots$ & $\ldots$ & $\ldots$ & $\ldots$ & . \\
\hline BM5bm2 & $\ldots$ & $\ldots$ & $\ldots$ & $\ldots$ & $\ldots$ & $\ldots$ & . \\
\hline BM6bm6 & $\ldots$ & $\ldots$ & $\ldots$ & $\ldots$ & $\ldots$ & $\ldots$ & . \\
\hline BN1bn1 & $\ldots$ & $\ldots$ & $\ldots$ & $\ldots$ & $\ldots$ & $\ldots$ & . \\
\hline BN1bn2 & $\ldots$ & $\ldots$ & $\ldots$ & $\ldots$ & $\ldots$ & $\ldots$ & . \\
\hline BN2bn1 & $\ldots$ & $\ldots$ & $\ldots$ & $\ldots$ & $\ldots$ & $\ldots$ & . \\
\hline BN3bn1 & $\ldots$ & $\ldots$ & $\ldots$ & $\ldots$ & $\ldots$ & $\ldots$ & . \\
\hline BK1bk1 & $\ldots$ & $\ldots$ & $\ldots$ & $\ldots$ & $\ldots$ & $\ldots$ & . \\
\hline BK2bk2 & $\ldots$ & $\ldots$ & $\ldots$ & $\ldots$ & $\ldots$ & $\ldots$ & . \\
\hline A1a1 & $\ldots$ & $\ldots$ & $\ldots$ & $\ldots$ & $\ldots$ & $\ldots$ & . \\
\hline A2a1 & $\ldots$ & $\ldots$ & $\ldots$ & $\ldots$ & $\ldots$ & $\ldots$ & . \\
\hline A3a1 & $\ldots$ & $\ldots$ & $\ldots$ & $\ldots$ & $\ldots$ & $\ldots$ & . \\
\hline A4a2 & $\ldots$ & $\ldots$ & $\ldots$ & $\ldots$ & $\ldots$ & $\ldots$ & . \\
\hline CN1cn1 & $\ldots$ & $\ldots$ & $\ldots$ & $\ldots$ & $\ldots$ & $\ldots$ & . \\
\hline CN1cn2 & $\ldots$ & $\ldots$ & $\ldots$ & $\ldots$ & $\ldots$ & $\ldots$ & . \\
\hline CN1cn3 & $\ldots$ & $\ldots$ & $\ldots$ & $\ldots$ & $\ldots$ & $\ldots$ & . \\
\hline CN1Cs1 & $\ldots$ & $\ldots$ & $\ldots$ & $\ldots$ & $\ldots$ & $\ldots$ & . \\
\hline CN2cn1 & $\cdots$ & $\ldots$ & $\ldots$ & $\ldots$ & $\ldots$ & $\ldots$ & . \\
\hline CN3cn2 & $\ldots$ & $\ldots$ & $\ldots$ & $\ldots$ & $\ldots$ & $\ldots$ & . \\
\hline CN4cn2 & $\ldots$ & $\ldots$ & $\ldots$ & $\ldots$ & $\ldots$ & $\ldots$ & . \\
\hline CS1cs1 & $\ldots$ & $\ldots$ & $\ldots$ & $\ldots$ & $\ldots$ & $\ldots$ & . \\
\hline CS1cs2 & $\ldots$ & $\ldots$ & $\ldots$ & $\ldots$ & $\ldots$ & $\ldots$ & . \\
\hline CS2cs 2 & $\ldots$ & $\ldots$ & $\ldots$ & $\ldots$ & $\ldots$ & $\ldots$ & . \\
\hline CS3cs1 & $\ldots$ & $\ldots$ & $\ldots$ & $\cdots$ & $\ldots$ & $\ldots$ & . \\
\hline CS4cs 2 & $\ldots$ & $\ldots$ & $\ldots$ & . & $\ldots$ & $\ldots$ & . \\
\hline M.clamit & $\ldots$ & $\ldots$ & 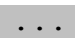 & $\ldots$ & $\ldots$ & 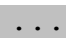 & A. \\
\hline M.phaeop & . . & . & $\ldots$ & . & . . & . . & . \\
\hline
\end{tabular}


Appendix 3.4 Sequenced individual Maoricicada campbelli COI, A6,A8 and TotalmtDNA haplotypes. Refer to Table 1 and Fig 1 for specific locality information.

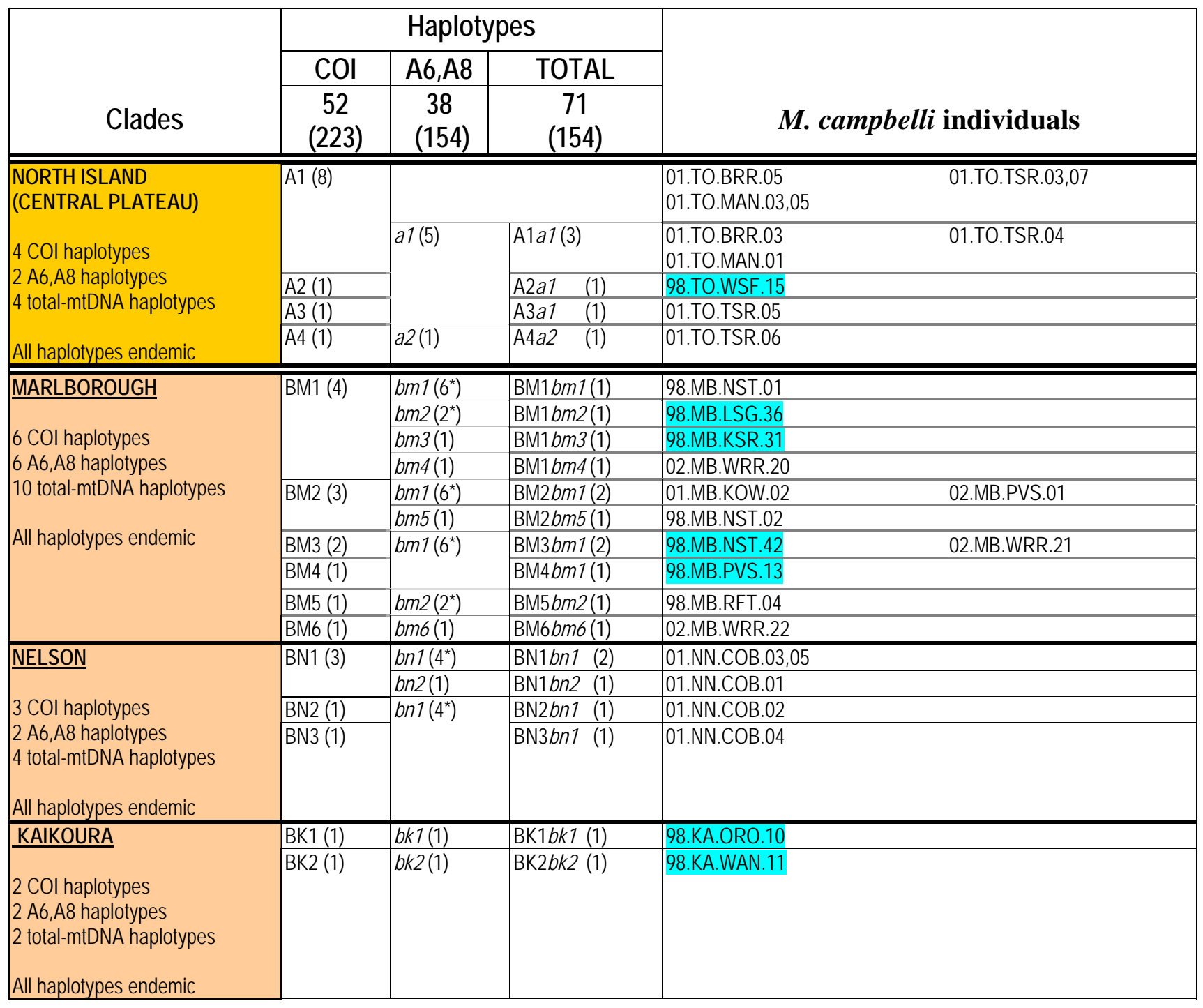




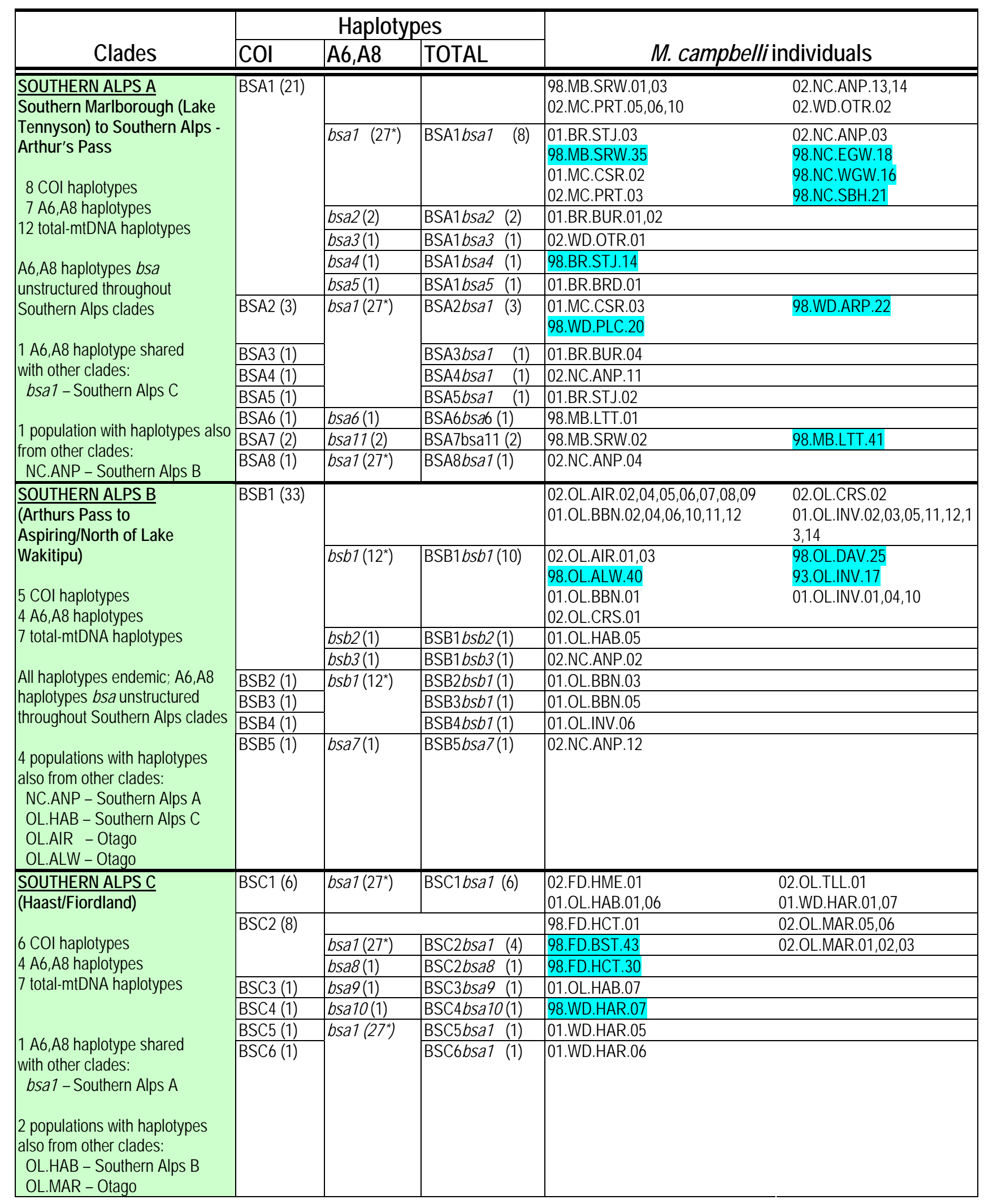




\begin{tabular}{|c|c|c|c|c|c|}
\hline \multirow{2}{*}{ Clades } & \multicolumn{3}{|c|}{ Haplotypes } & \multirow{2}{*}{\multicolumn{2}{|c|}{ M. campbelli individuals }} \\
\hline & $\mathrm{COI}$ & A6,A8 & TOTAL & & \\
\hline \multirow{14}{*}{$\begin{array}{l}\text { WAITAKI } \\
9 \text { COI haplotypes } \\
7 \text { A6,A8 haplotypes } \\
12 \text { total-mtDNA haplotypes } \\
\text { All haplotypes endemic } \\
\\
2 \text { populations with haplotypes } \\
\text { also from other clades: } \\
\text {-MK.LOH - Otago } \\
\text {-MK.OHA - Otago }\end{array}$} & \multirow[t]{4}{*}{ BW1 (37) } & & & $\begin{array}{l}\text { 01.CO.AWR.02 } \\
\text { 98.MK.HVI.02,03,04 } \\
\text { 01.MK.LOH.02,50,51 }\end{array}$ & $\begin{array}{l}\text { 02.MK.MCV.13 } \\
01 . M K . R H D .01,03,04,06,10 \text {, } \\
11,12,13,15\end{array}$ \\
\hline & & bw1 $\left(22^{*}\right)$ & BW1bw1 (17) & \begin{tabular}{|l|} 
98.MK.BCL.33 \\
01.MK.BNO.01 \\
98.MK.HVN.01 \\
98.MK.HVI.01 \\
01.MK.LOH.05,10,12,53,55\# \\
02.MK.LTM.31\# \\
\end{tabular} & $\begin{array}{l}\text { 98.MK.MCA.23 } \\
\text { 98.MK.MCB.27 } \\
\text { 02.MK.MCR.01 } \\
\text { 02.MK.MCV.12 } \\
\text { 02.MK.OHA.23 } \\
\text { 01.MK.RHD.02,05 } \\
\end{array}$ \\
\hline & & bw3 (3*) & BW1bw3 (2) & 01.CO.AWR.05,07 & \\
\hline & & bw5 (1) & BW1bw5 (1) & 98.CO.AWR.32 & \\
\hline & \multirow[t]{2}{*}{ BW2 (2) } & bw1 $\left(22^{*}\right)$ & BW2bw1 (1) & 01.MK.LOH.06 & \\
\hline & & bw3 $\left(3^{*}\right)$ & BW2bw3 (1) & 01.CO.AWR.04 & \\
\hline & BW3 (2) & bw2 $\left(3^{*}\right)$ & BW3bw2 (2) & 01.SC.BWT.01,02 & \\
\hline & BW4 (1) & bw1 $\left(22^{*}\right)$ & BW4bw1 (1) & 01.MK.LOH.56\# & \\
\hline & BW5 (1) & bw4 (1) & BW5bw4 (1) & 98.CO.KYE.29 & \\
\hline & BW6 (1) & \multirow[t]{2}{*}{ bw1 (22*) } & BW6bw1 (1) & 01.MK.RHD.14 & \\
\hline & BW7 (2) & & BW7bw1 (2) & 01.CO.AWR.03 & \multirow[t]{4}{*}{ 98.CO.OTA.01 } \\
\hline & BW8 (1) & bw6 (1) & BW8bw6 (1) & 01.CO.AWR.06 & \\
\hline & BW9 (1) & bw7 (1) & BW9bw7 (1) & 02.MC.SCR.01 & \\
\hline & BW10(1) & $b w 2\left(3^{*}\right)$ & BW10bw2 (1) & 98.CO.OTA.02 & \\
\hline \multirow{8}{*}{$\begin{array}{l}\text { OTAGO NORTH } \\
4 \text { COI haplotypes } \\
3 \text { A6,A8 haplotypes } \\
7 \text { total-mtDNA haplotypes } \\
\text { All haplotypes endemic } \\
1 \text { populations with haplotypes } \\
\text { also from another clade: } \\
\text {-MK.LOH - Waitaki }\end{array}$} & \multirow[t]{5}{*}{ CN1(33) } & & & $\begin{array}{l}\text { 01.CO.CRA.05 } \\
\text { 01.CO.OMR.02 }\end{array}$ & $\begin{array}{l}\text { 01.MK.AHU.03,04,10 } \\
\text { 01.MK.LOH.01,04,08,52,54 }\end{array}$ \\
\hline & & $\operatorname{cn} 1\left(20^{*}\right)$ & CN1cn1 (18) & \begin{tabular}{|l|} 
01.CO.CRA.03 \\
98.CO.LTO.38 \\
01.CO.OMR.01 \\
98.MK.AHU.44 \\
01.MK.AHU.01,02,11,12,13\#
\end{tabular} & $\begin{array}{l}\text { 98.MK.LIN.28 } \\
\text { 01.MK.LIN.01,02,10 } \\
\text { 01.MK.LOH.03,07,09,16 } \\
\text { 02.MK.OHA.24 }\end{array}$ \\
\hline & & $\operatorname{cs1}\left(11^{*}\right)$ & Cn1cs1 & 01.OL.TWE.04 & \\
\hline & & $\frac{\operatorname{cn} 2\left(4^{*}\right)}{4}$ & CN1cn2 & 98.OL.NLW.08 & 98.CO.ABT.09 \\
\hline & & cn3 (2) & CN1cn3 & 01.MK.LOH.15 & 98.MK.LOH.37 \\
\hline & CN2 (2) & $\operatorname{cn} 1\left(20^{\star}\right)$ & CN2cn1 & 01.MK.LOH.11,13 & \\
\hline & CN3 (1) & $\operatorname{cn} 2\left(4^{\star}\right)$ & CN3cn2 & 01.CO.CRA.09 & \\
\hline & CN4 (1) & & CN4cn2 & 01.CO.CRA.10 & \\
\hline OTAGO SOUTH & CS1 (17) & & & 02.OL.FRL.03,08 & OL.TWE.06,.07,10 \\
\hline $\begin{array}{l}4 \text { COI haplotypes } \\
2 \text { A6,A8 haplotypes } \\
5 \text { total-mtDNA haplotypes }\end{array}$ & & $\operatorname{cs1}\left(11^{\star}\right)$ & CS1cs1 & $\begin{array}{l}\text { 01.CO.RSA.01, } \\
\text { 98.CO.RSA.19 } \\
\text { 02.OL.AIR.10 } \\
\text { 98.OL.ALW.02 }\end{array}$ & $\begin{array}{l}\text { 02.OL.FRL.01,06 } \\
\text { 98.OL.TWE.34 } \\
\text { 01.OL.TWE.01,02,03 }\end{array}$ \\
\hline & & $\operatorname{cs2}\left(5^{\star}\right)$ & CS1cs2 & 02.OL.ORE.01 & 02.OL.MAR.04 \\
\hline All haplotypes endemic & CS2 (2) & & CS2cs2 & 02.OL.EYR.01 & 02.OL.FRL.10 \\
\hline 2 nonulationc with hanlntynoc & $\operatorname{CS3}(1)$ & $\operatorname{cs} 1\left(11^{\star}\right)$ & CS3cs1 (1) & 98.CO.OMR.24 & \\
\hline $\begin{array}{l}\text { Slso from other clades: } \\
\text {-OL.AIR - Southern Alps B } \\
\text {-OL.ALW - Southern Alps B } \\
\text {-OL.MAR - Southern Alps C }\end{array}$ & CS4 (1) & $\operatorname{cs} 2\left(5^{*}\right)$ & CS4cs2 & 02.OL.EYR.02 & \\
\hline
\end{tabular}

All numbers in brackets stand for the number of individuals sequenced for that haplotype.

* denotes A6-A8 haplotype elsewhere on table.

All individuals are male unless followed by female symbol (\#)

Highlighting denotes individuals sequenced by Buckley et al. 2001a. These populations have been renamed with locality codes, the individual number after the code is the same as the original population number published. 
Appendix 3.5 Geodis 2.0 input and output for COI and Total-mtDNA analyses of Maoricicada campbelli haplotypes. All output based on 10000 resamples.

\section{Population parameters used for all analyses:}

number, population code

number of individuals in that population, coordinates (degrees, minutes, decimal seconds)

70 populations

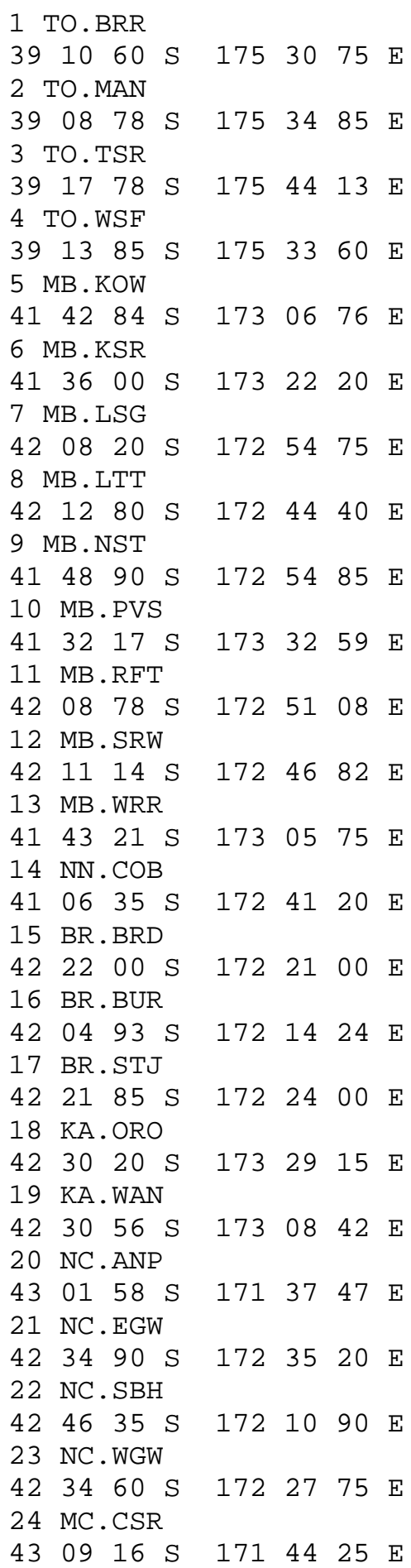

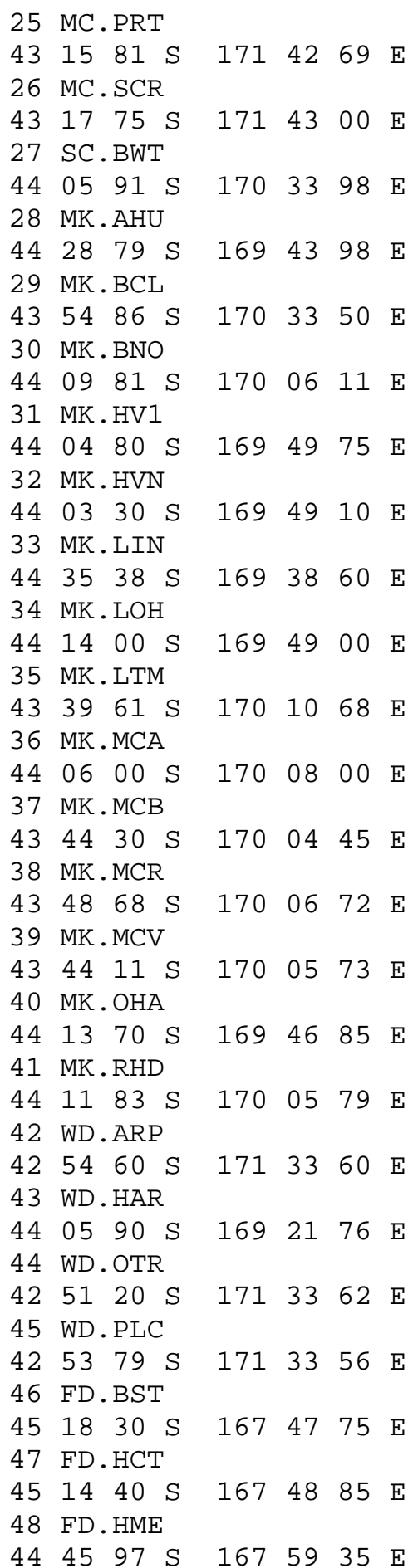

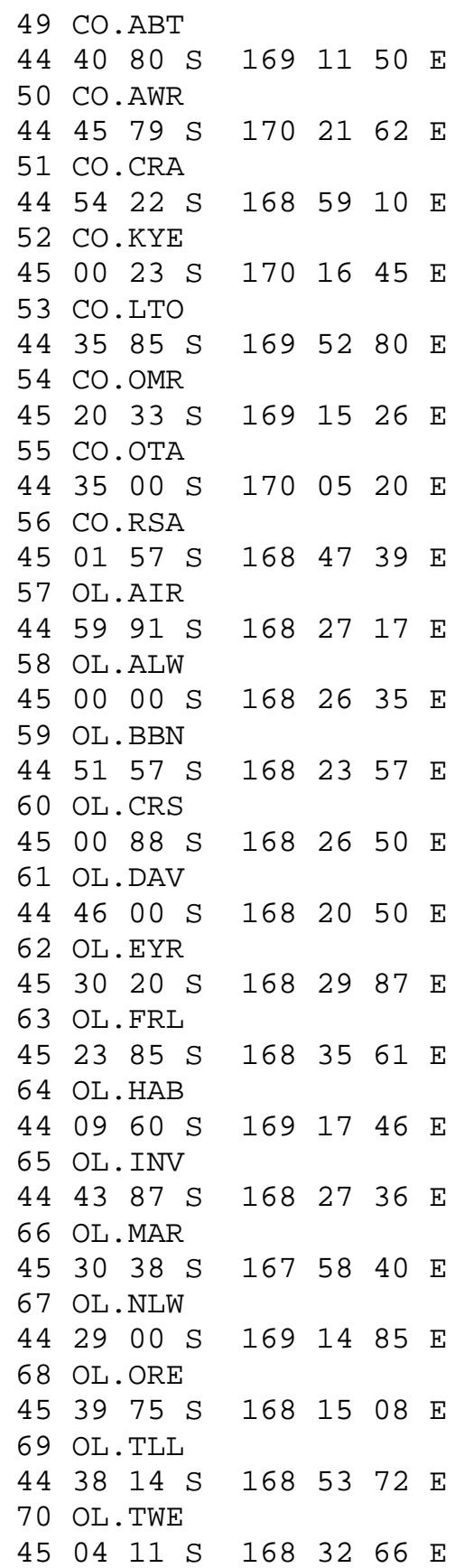




\section{M. campbelli COI Geodis 2.0 input matrices:}

21

1-1 SOUTHERN ALPS A (1)

7

BSA1 BSA2 BSA3 BSA4 BSA5 BSA6 BSA8

\begin{tabular}{|c|c|c|c|c|c|c|c|c|c|c|c|c|}
\hline $\begin{array}{r}0 \\
14\end{array}$ & 1 & & 1 & \multicolumn{2}{|c|}{1} & \multicolumn{2}{|l|}{1} & 1 & \multicolumn{2}{|c|}{1} & & \\
\hline 812 & 15 & 16 & 17 & 20 & 21 & 22 & 23 & 24 & 25 & 42 & 44 & 45 \\
\hline 3 & 1 & 2 & 2 & 3 & 1 & 1 & 1 & 1 & 4 & $\odot$ & 2 & 0 \\
\hline 0 & 0 & $\odot$ & $\odot$ & $\odot$ & $\odot$ & 0 & $\odot$ & 1 & $\odot$ & 1 & $\odot$ & 1 \\
\hline$\odot$ & $\odot$ & 1 & $\odot$ & $\odot$ & $\odot$ & $\odot$ & $\odot$ & $\odot$ & $\odot$ & $\odot$ & $\odot$ & $\odot$ \\
\hline$\odot$ & $\odot$ & $\odot$ & $\odot$ & 1 & $\odot$ & $\odot$ & $\odot$ & $\odot$ & $\odot$ & $\odot$ & $\odot$ & $\odot$ \\
\hline 0 & 0 & $\odot$ & 1 & 0 & $\odot$ & 0 & $\odot$ & $\odot$ & $\odot$ & $\odot$ & $\odot$ & $\odot$ \\
\hline$\odot$ & $\odot$ & $\odot$ & $\odot$ & $\odot$ & $\odot$ & 0 & $\odot$ & $\odot$ & $\odot$ & $\odot$ & $\odot$ & $\odot$ \\
\hline$\odot$ & $\odot$ & $\odot$ & $\odot$ & 1 & $\odot$ & $\odot$ & $\odot$ & $\odot$ & $\odot$ & $\odot$ & $\odot$ & $\odot$ \\
\hline
\end{tabular}

1-3 SOUTHERN ALPS B (2)

5

BSB1 BSB2 BSB3 BSB4 BSB5

$\begin{array}{lllll}\odot & 1 & 1 & 1 & 1\end{array}$

8

$\begin{array}{llllllll}20 & 57 & 58 & 59 & 60 & 61 & 64 & 65\end{array}$

$\begin{array}{llllllll}1 & 9 & 1 & 7 & 2 & 1 & 1 & 11\end{array}$

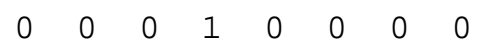

$\begin{array}{llllllll}\odot & 0 & 0 & 1 & 0 & 0 & 0 & 0\end{array}$

$\begin{array}{llllllll} & 0 & 0 & 0 & 0 & 0 & 0 & 1\end{array}$

$\begin{array}{llllllll}1 & \odot & \odot & \odot & \odot & 0 & \odot & 0\end{array}$

1-4 SOUTHERN ALPS C (3)

4

BSC1 BSC2 BSC3 BSC4

\begin{tabular}{rrrrrrr}
$\odot$ & & 1 & 1 & \multicolumn{1}{c}{1} \\
7 & & & & & \\
43 & 46 & 47 & 48 & 64 & 66 & 69 \\
2 & $\odot$ & $\odot$ & 1 & 2 & $\odot$ & 1 \\
0 & 1 & 2 & $\odot$ & 0 & 5 & 0 \\
$\odot$ & $\odot$ & 0 & $\odot$ & 1 & $\odot$ & 0 \\
1 & $\odot$ & $\odot$ & $\odot$ & $\odot$ & $\odot$ & $\odot$ \\
$1-6$ & WAITAKI & $\# 1$ & $(4)$ & \\
6 & & & &
\end{tabular}

BW1 BW2 BW4 BW5 BW6 BW7

$\begin{array}{llllll}\odot & 1 & 1 & 1 & 1 & 1\end{array}$

15

$\begin{array}{lllllllllllllll}29 & 30 & 31 & 32 & 34 & 35 & 36 & 37 & 38 & 39 & 40 & 41 & 50 & 52 & 55\end{array}$

$\begin{array}{lllllllllllllll}1 & 1 & 4 & 1 & 8 & 1 & 1 & 1 & 1 & 2 & 1 & 11 & 4 & 0 & 0\end{array}$

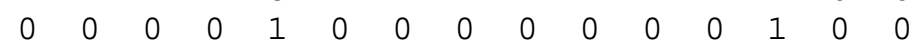

$\begin{array}{lllllllllllllll} & 0 & 0 & 0 & 1 & 0 & 0 & 0 & 0 & 0 & 0 & 0 & 0 & 0 & 0\end{array}$

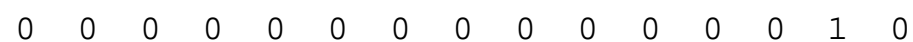

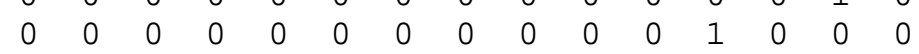

$\begin{array}{lllllllllllllll}\odot & 0 & \odot & \odot & 0 & 0 & \odot & 0 & 0 & 0 & \odot & 0 & 1 & 0 & 1\end{array}$

1-7 WAITAKI \#2 (5)

2

BW3 BW10

○ 1

2

2755

20

01

1-10 MARLBOROUGH (6)

4

BM1 BM2 BM3 BM5

\begin{tabular}{rrrrrrr}
$\odot$ & & 0 & 1 & \multicolumn{1}{c}{1} \\
7 & & & & & \\
5 & 6 & 7 & 9 & 10 & 11 & 13 \\
$\odot$ & 1 & 1 & 1 & $\odot$ & $\odot$ & 1
\end{tabular}


$\begin{array}{lllllll}1 & 0 & 0 & 1 & 1 & 0 & 0 \\ 0 & 0 & 0 & 1 & 0 & 0 & 1\end{array}$

$\begin{array}{lllllll}\odot & 0 & 0 & 0 & 0 & 1 & 0\end{array}$

1-16 NORTH ISLAND (7)

4

A1 A2 A3 A4

$\begin{array}{llll}\odot & 1 & 1 & 1\end{array}$

4

1234

2330

$\begin{array}{lllll}0 & 0 & 0 & 1\end{array}$

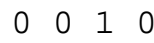

$\odot \odot 10$

1-17 NORTH OTAGO (8)

3

CN1 CN2 CN3

$\begin{array}{lll}\odot & 1 & 1\end{array}$

10

$\begin{array}{llllllllll}28 & 33 & 34 & 40 & 49 & 51 & 53 & 54 & 67 & 70\end{array}$

$\begin{array}{llllllllll}9 & 4 & 11 & 1 & 1 & 2 & 1 & 2 & 1 & 1\end{array}$

$\begin{array}{llllllllll}0 & 0 & 2 & 0 & 0 & 0 & 0 & 0 & 0 & 0\end{array}$

$\begin{array}{llllllllll}\odot & \odot & \odot & \odot & \odot & 1 & \odot & \odot & \odot & \odot\end{array}$

1-19 SOUTH OTAGO \#1 (9)

2

CS1 CS3

○ 1

6

$\begin{array}{llllll}54 & 56 & 57 & 58 & 63 & 70\end{array}$

$\begin{array}{llllll}0 & 2 & 1 & 1 & 4 & 7\end{array}$

$\begin{array}{llllll}1 & 0 & 0 & 0 & 0 & 0\end{array}$

1-20 SOUTH OTAGO \#2 (10)

2

CS2 CS4

○ 1

2

6263

11

10

2-1 SOUTHERN ALPS A (11)

2

1-1 1-2

$\odot 1$

14

$\begin{array}{llllllllllllll}8 & 12 & 15 & 16 & 17 & 20 & 21 & 22 & 23 & 24 & 25 & 42 & 44 & 45\end{array}$

$\begin{array}{llllllllllllll}1 & 3 & 1 & 3 & 3 & 5 & 1 & 1 & 1 & 1 & 4 & 1 & 2 & 1\end{array}$

$\begin{array}{llllllllllllll}1 & 1 & 0 & 0 & \odot & 0 & 0 & 0 & 0 & 0 & 0 & 0 & 0 & 0\end{array}$

2-3 SOUTHERN ALPS C (12)

2

1-4 1-5

01

7

$\begin{array}{lllllll}43 & 46 & 47 & 48 & 64 & 66 & 69\end{array}$

$\begin{array}{lllllll}3 & 1 & 2 & 1 & 3 & 5 & 1\end{array}$

$\begin{array}{lllllll}2 & 0 & 0 & 0 & 0 & 0 & 0\end{array}$

2-4 WAITAKI (13)

4

1-6 $1-7$ 1-8 1 -9

$\begin{array}{llll}0 & 1 & 1 & 1\end{array}$

17

$\begin{array}{lllllllllllllllll}26 & 27 & 29 & 30 & 31 & 32 & 34 & 35 & 36 & 37 & 38 & 39 & 40 & 41 & 50 & 52 & 55\end{array}$

$\begin{array}{lllllllllllllllll}0 & 0 & 1 & 1 & 4 & 1 & 10 & 1 & 1 & 1 & 1 & 2 & 1 & 12 & 6 & 1 & 1\end{array}$

$\begin{array}{lllllllllllllllll}0 & 2 & 0 & 0 & 0 & 0 & 0 & 0 & 0 & 0 & 0 & 0 & 0 & 0 & 0 & 0 & 1\end{array}$

$\begin{array}{lllllllllllllllll}0 & 0 & 0 & 0 & 0 & 0 & 0 & 0 & 0 & 0 & 0 & 0 & 0 & 0 & 1 & 0 & 0\end{array}$

$\begin{array}{cccccccccc}1 & \odot & \odot & \odot & \odot & \odot & \odot & \odot & \odot & \odot \\ \mathbf{2 - 5} & \text { MARLBOROUGH } & + & \text { NELSON } & (14)\end{array}$ 
4

1-10 1-11 1-12 1-13

$\begin{array}{llll}0 & 1 & 1 & 1\end{array}$

8

$\begin{array}{llllllll}5 & 6 & 7 & 9 & 10 & 11 & 13 & 14\end{array}$

$\begin{array}{llllllll}1 & 1 & 1 & 3 & 1 & 1 & 2 & 0\end{array}$

$\begin{array}{llllllll}0 & 0 & 0 & 0 & 1 & 0 & 0 & 0\end{array}$

$\begin{array}{llllllll}0 & 0 & 0 & 0 & 0 & 0 & 1 & 0\end{array}$

$\odot \begin{array}{llllllll}\odot & \odot & \odot & \odot & \odot & \odot & \odot & 5\end{array}$

2-6 KAIKOURA (15)

2

1-14 1-15

10

2

1819

10

01

2-8 NORTH OTAGO (16)

2

$1-17 \quad 1-18$

$0 \quad 1$

10

$\begin{array}{llllllllll}28 & 33 & 34 & 40 & 49 & 51 & 53 & 54 & 67 & 70\end{array}$

$\begin{array}{rrrrrrrrrr}9 & 4 & 13 & 1 & 1 & 3 & 1 & 2 & 1 & 1\end{array}$

$\begin{array}{llllllllll}\odot & \odot & \odot & \odot & \odot & 1 & \odot & \odot & \odot & \odot\end{array}$

2-9 SOUTH OTAGO (17)

2

$1-191-20$

01

7

$\begin{array}{lllllll}54 & 56 & 57 & 58 & 62 & 63 & 70\end{array}$

$\begin{array}{lllllll}1 & 2 & 1 & 1 & 0 & 4 & 7\end{array}$

$\odot \begin{array}{lllllll} & 0 & \odot & 0 & 2 & 1 & 0\end{array}$

3-1 SOUTHERN ALPS + WAITAKI (18)

4

$\begin{array}{lllll}2-1 & 2-2 & 2-3 & 2-4\end{array}$

0
44

$\begin{array}{llllllllllllllllllllllllllll}8 & 12 & 15 & 16 & 17 & 20 & 21 & 22 & 23 & 24 & 25 & 26 & 27 & 29 & 30 & 31 & 32 & 34 & 35 & 36 & 37 & 38 & 39 & 40 & 41 & 42 & 43 & 44\end{array}$

$\begin{array}{llllllllllllllllllllllllllllll}2 & 4 & 1 & 3 & 3 & 5 & 1 & 1 & 1 & 1 & 4 & \odot & \odot & 0 & \odot & \odot & \odot & \odot & \odot & \odot & \odot & 0 & \odot & 0 & 0 & 1 & 0 & 2\end{array}$

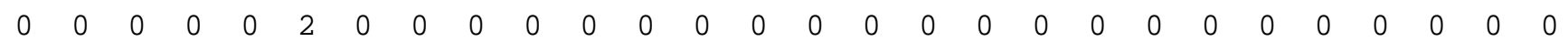

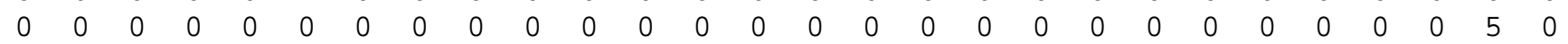

$\odot \begin{array}{llllllllllllllllllllllllllllll} & \odot & \odot & \odot & \odot & \odot & \odot & \odot & \odot & \odot & 1 & 2 & 1 & 1 & 4 & 1 & 1 & 0 & 1 & 1 & 1 & 1 & 2 & 1 & 12 & 0 & \odot & \odot & \odot\end{array}$

$\begin{array}{llllllllllllllll}45 & 46 & 47 & 48 & 50 & 52 & 55 & 57 & 58 & 59 & 60 & 61 & 64 & 65 & 66 & 69\end{array}$

$\begin{array}{llllllllllllllll}1 & \odot & \odot & \odot & \odot & \odot & \odot & \odot & \odot & \odot & \odot & \odot & \odot & \odot & \odot & 0\end{array}$

$\begin{array}{llllllllllllllll}\odot & \odot & \odot & \odot & \odot & \odot & \odot & 9 & 1 & 9 & 2 & 1 & 1 & 12 & \odot & 0\end{array}$

$\begin{array}{llllllllllllllll}\odot & 1 & 2 & 1 & 3 & 5 & 1 & \odot & \odot & \odot & \odot & \odot & 3 & \odot & 5 & 1\end{array}$

$\begin{array}{llllllllllllllll}\odot & \odot & \odot & 0 & 7 & 1 & 2 & \odot & \odot & 0 & \odot & 0 & \odot & \odot & 0 & 0\end{array}$

3-2 NORTHERN SOUTH ISLAND (19)

2

$2-52-6$

10

$\begin{array}{llllllllll}5 & 6 & 7 & 9 & 10 & 11 & 13 & 14 & 18 & 19\end{array}$

$\begin{array}{llllllllll}1 & 1 & 1 & 3 & 2 & 1 & 3 & 5 & 0 & 0\end{array}$

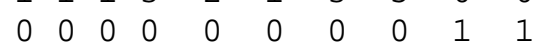

3-4 OTAGO (20)

2

$2-8 \quad 2-9$

$0 \quad 0$

15

$\begin{array}{lllllllllllllll}28 & 33 & 34 & 40 & 49 & 51 & 53 & 54 & 56 & 57 & 58 & 62 & 63 & 67 & 70\end{array}$

$\begin{array}{lllllllllllllll}9 & 4 & 13 & 1 & 1 & 4 & 1 & 2 & \odot & \odot & \odot & \odot & \odot & 1 & 1\end{array}$

$\odot \begin{array}{lllllllllllllll}\odot & \odot & \odot & \odot & 0 & \odot & 0 & 1 & 2 & 1 & 1 & 2 & 1 & 0 & 7\end{array}$

4-1 SOUTH ISLAND (NOT OTAGO) (21) 
$\begin{array}{lllllllllllllllllllllllllllll}5 & 6 & 7 & 8 & 9 & 10 & 11 & 12 & 13 & 14 & 15 & 16 & 17 & 18 & 19 & 20 & 21 & 22 & 23 & 24 & 25 & 26 & 27 & 29 & 30 & 31 & 32 & 34 & 35\end{array}$

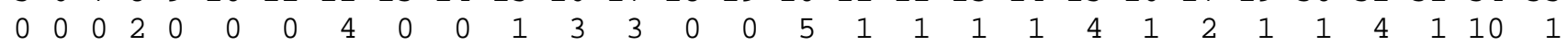
$\begin{array}{lllllllllllllllllllllllllllllll}1 & 1 & 1 & \odot & 3 & 2 & 1 & \odot & 3 & 5 & \odot & \odot & 0 & 1 & 1 & \odot & \odot & 0 & 0 & 0 & 0 & \odot & 0 & 0 & 0 & 0 & 0 & 0 & 0\end{array}$ $\begin{array}{llllllllllllllllllllllll}36 & 37 & 38 & 39 & 40 & 41 & 42 & 43 & 44 & 45 & 46 & 47 & 48 & 50 & 52 & 55 & 57 & 58 & 59 & 60 & 61 & 64 & 66 & 69\end{array}$ $\begin{array}{llllllllllllllllllllllll}1 & 1 & 1 & 2 & 1 & 12 & 1 & 5 & 2 & 1 & 1 & 2 & 1 & 10 & 6 & 3 & 9 & 1 & 9 & 2 & 1 & 4 & 5 & 1\end{array}$

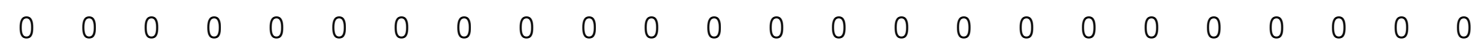

END

\section{M. campbelli Geodis COI analysis output results:}

\section{PERMUTATION ANALYSIS OF 1-1 SOUTHERN ALPS B (1)}

PART I. EXACT CONTINGENCY TEST: OBSERVED CHI-SQUARE STATISTIC $=79.9111$

THE PROBABILITY OF A RANDOM CHI-SQUARE BEING GREATER THAN OR EQUAL TO THE OBSERVED CHI-SQUARE $=0.4892$

PART II. GEOGRAPHIC DISTANCE ANALYSIS:

\begin{tabular}{|c|c|c|c|c|}
\hline \multirow{4}{*}{$\begin{array}{r}\text { CLADE } \\
\text { TYPE }\end{array}$} & BSA1 (Interior) & & & \\
\hline & OF DISTANCE & DISTANCE & PROB. $<=$ & PROB. $>=$ \\
\hline & WITHIN CLADE & 46.5923 & 0.0800 & $\odot .9200$ \\
\hline & NESTED CLADE & 48.2494 & $\odot .1897$ & $\odot .8103$ \\
\hline \multirow{4}{*}{$\begin{array}{r}\text { CLADE } \\
\text { TYPE }\end{array}$} & BSA2 (Tip) & & & \\
\hline & OF DISTANCE & DISTANCE & PROB. $<=$ & PROB. $>=$ \\
\hline & WITHIN CLADE & 9.7724 & 0.0598 & $\odot .94 \odot 2$ \\
\hline & NESTED CLADE & 51.1969 & 0.4122 & 0.5878 \\
\hline \multirow{4}{*}{$\begin{array}{r}\text { CLADE } \\
\text { TYPE }\end{array}$} & BSA3 (Tip) & & & \\
\hline & OF DISTANCE & DISTANCE & PROB. $<=$ & PROB. $>=$ \\
\hline & WITHIN CLADE & $\odot .000 \odot$ & 0.8651 & 1.0000 \\
\hline & NESTED CLADE & 67.0174 & 0.7213 & 0.3811 \\
\hline \multirow{4}{*}{$\begin{array}{r}\text { CLADE } \\
\text { TYPE }\end{array}$} & BSA4 (Tip) & & & \\
\hline & OF DISTANCE & DISTANCE & PROB. $<=$ & PROB. $>=$ \\
\hline & WITHIN CLADE & $\odot .0000$ & 0.8635 & 1.0000 \\
\hline & NESTED CLADE & 53.5540 & 0.5465 & 0.4535 \\
\hline \multirow{4}{*}{$\begin{array}{r}\text { CLADE } \\
\text { TYPE }\end{array}$} & BSA5 (Tip) & & & \\
\hline & OF DISTANCE & DISTANCE & PROB. $<=$ & PROB . $>=$ \\
\hline & WITHIN CLADE & $\odot .00 \odot \odot$ & 0.8652 & 1.0000 \\
\hline & NESTED CLADE & 43.1348 & 0.2390 & 0.8612 \\
\hline \multirow{4}{*}{$\begin{array}{r}\text { CLADE } \\
\text { TYPE }\end{array}$} & BSA6 (Tip) & & & \\
\hline & OF DISTANCE & DISTANCE & PROB. $<=$ & PROB. $>=$ \\
\hline & WITHIN CLADE & $\odot .0000$ & 0.8711 & 1.0000 \\
\hline & NESTED CLADE & 74.5877 & 0.8645 & 0.1355 \\
\hline \multirow{4}{*}{$\begin{array}{r}\text { CLADE } \\
\text { TYPE }\end{array}$} & BSA8 (Tip) & & & \\
\hline & OF DISTANCE & DISTANCE & PROB. $<=$ & PROB. $>=$ \\
\hline & WITHIN CLADE & $\odot .0000$ & 0.8597 & 1.0000 \\
\hline & NESTED CLADE & 53.5540 & 0.5555 & 0.4445 \\
\hline
\end{tabular}

PART III. TEST OF INTERIOR VS. TIP CLADES:

$\begin{array}{crrr}\text { TYPE OF DISTANCE } & \text { I-T DISTANCE } & \text { PROB. }<= & \text { PROB. }>= \\ \text { WITHIN CLADE } & 42.9276 & 0.8183 & 0.1817 \\ \text { NESTED CLADE } & -7.4305 & 0.3960 & 0.6040\end{array}$


PERMUTATION ANALYSIS OF 1-3 SOUTHERN ALPS B (2)

PART I. EXACT CONTINGENCY TEST: OBSERVED CHI-SQUARE STATISTIC =

THE PROBABILITY OF A RANDOM CHI-SQUARE BEING GREATER THAN OR EQUAL TO THE OBSERVED CHI-SQUARE $=\quad 0.3967$

PART II. GEOGRAPHIC DISTANCE ANALYSIS:

\begin{tabular}{|c|c|c|c|c|}
\hline \multirow{4}{*}{$\begin{array}{r}\text { CLADE } \\
\text { TYPE }\end{array}$} & \multicolumn{4}{|l|}{ BSB1 (Interior) } \\
\hline & OF DISTANCE & DISTANCE & PROB . $<=$ & PROB . $>=$ \\
\hline & WITHIN CLADE & 29.1220 & 0.1333 & 0.8667 \\
\hline & NESTED CLADE & 33.4517 & 0.1544 & $\odot .8456$ \\
\hline \multicolumn{5}{|c|}{ BSB2 (Tip) } \\
\hline \multirow[t]{3}{*}{ TYPE } & OF DISTANCE & DISTANCE & PROB . $<=$ & PROB . > = \\
\hline & WITHIN CLADE & $0.000 \odot$ & 0.7575 & 1.0000 \\
\hline & NESTED CLADE & 19.8770 & $\odot .5650$ & $\odot .6775$ \\
\hline \multirow{4}{*}{$\begin{array}{r}\text { CLADE } \\
\text { TYPE }\end{array}$} & BSB3 (Tip) & & & \\
\hline & OF DISTANCE & DISTANCE & PROB . $<=$ & PROB . > = \\
\hline & WITHIN CLADE & $\odot .00 \odot \odot$ & 0.7582 & 1.0000 \\
\hline & NESTED CLADE & 19.8770 & 0.5705 & $\odot .6713$ \\
\hline \multirow{4}{*}{$\begin{array}{r}\text { CLADE } \\
\text { TYPE }\end{array}$} & BSB4 (Tip) & & & \\
\hline & OF DISTANCE & DISTANCE & PROB . $<=$ & PROB . > = \\
\hline & WITHIN CLADE & 0.0000 & 0.7656 & 1.0000 \\
\hline & NESTED CLADE & 12.3973 & 0.3312 & 1.0000 \\
\hline \multirow{4}{*}{$\begin{array}{r}\text { CLADE } \\
\text { TYPE }\end{array}$} & BSB5 (Tip) & & & \\
\hline & OF DISTANCE & DISTANCE & PROB . $<=$ & PROB. $>=$ \\
\hline & WITHIN CLADE & 0.0000 & 0.7545 & 1.0000 \\
\hline & NESTED CLADE & 309.1238 & 1.0000 & 0.0527 \\
\hline
\end{tabular}

PART III. TEST OF INTERIOR VS. TIP CLADES:

$\begin{array}{rrrr}\text { TYPE OF DISTANCE } & \text { I-T DISTANCE } & \text { PROB. }<= & \text { PROB. }>= \\ \text { WITHIN CLADE } & 29.1220 & 0.1483 & 0.8517 \\ \text { NESTED CLADE } & -56.8671 & 0.1747 & 0.8253\end{array}$

PERMUTATION ANALYSIS OF 1-4 SOUTHERN APLS C (3)

PART I. EXACT CONTINGENCY TEST: OBSERVED CHI-SQUARE STATISTIC = 23.1111

THE PROBABILITY OF A RANDOM CHI-SQUARE BEING GREATER THAN OR EQUAL TO THE OBSERVED CHI-SQUARE $=\quad 0.3141$

PART II. GEOGRAPHIC DISTANCE ANALYSIS:

\begin{tabular}{|c|c|c|c|c|}
\hline \multirow{4}{*}{$\begin{array}{r}\text { CLADE } \\
\text { TYPE }\end{array}$} & \multicolumn{4}{|l|}{ BSC1 (Interior) } \\
\hline & OF DISTANCE & DISTANCE & PROB . $<=$ & PROB . $>=$ \\
\hline & WITHIN CLADE & 48.5242 & 0.1451 & ๑.8549 \\
\hline & NESTED CLADE & 62.6613 & 0.3081 & ๑. 6919 \\
\hline \multicolumn{5}{|c|}{ BSC2 (Tip) } \\
\hline \multirow[t]{3}{*}{ TYPE } & OF DISTANCE & DISTANCE & PROB . <= & PROB . $>=$ \\
\hline & WITHIN CLADE & 12.2653 & $\odot .0 \odot \odot \odot$ & 1.0000 \\
\hline & NESTED CLADE & 66.7235 & 0.4447 & $\odot .5553$ \\
\hline \multirow{4}{*}{$\begin{array}{r}\text { CLADE } \\
\text { TYPE }\end{array}$} & BSC3 (Tip) & & & \\
\hline & OF DISTANCE & DISTANCE & PROB . <= & PROB . $>=$ \\
\hline & WITHIN CLADE & $\odot . \odot \odot \odot \odot$ & $1 . \odot \odot \odot \odot$ & $1.0 \odot \odot \odot$ \\
\hline & NESTED CLADE & 107.5348 & 0.6190 & 0.3810 \\
\hline
\end{tabular}


CLADE BSC4 (Tip)

TYPE OF DISTANCE WITHIN CLADE

DISTANCE

○. 0000

NESTED CLADE

$$
\text { PROB . }<=
$$

1.0000

$\odot .8116$

PROB . > =

1.0000

$\odot .1884$

PART III. TEST OF INTERIOR VS. TIP CLADES:

$\begin{array}{rrrr}\text { TYPE OF DISTANCE } & \text { I-T DISTANCE } & \text { PROB. }<= & \text { PROB. }>= \\ \text { WITHIN CLADE } & 38.7120 & 0.9109 & 0.0891 \\ \text { NESTED CLADE } & -13.1063 & 0.2855 & 0.7145\end{array}$

PERMUTATION ANALYSIS OF 1-6 WAITAKI \#1 (4)

PART I. EXACT CONTINGENCY TEST: OBSERVED CHI-SQUARE STATISTIC = 79.0216

THE PROBABILITY OF A RANDOM CHI-SQUARE BEING GREATER THAN OR EQUAL TO THE OBSERVED CHI-SQUARE $=\quad 0.3884$

PART II. GEOGRAPHIC DISTANCE ANALYSIS:

CLADE BW1 (Interior)

TYPE OF DISTANCE WITHIN CLADE

DISTANCE

29.2423

30.3618

NESTED CLADE

CLADE BW2 (Tip)

TYPE OF DISTANCE

WITHIN CLADE

NESTED CLADE

CLADE BW4 (Tip)

TYPE OF DISTANCE WITHIN CLADE NESTED CLADE

CLADE BW5 (Tip)

TYPE OF DISTANCE WITHIN CLADE NESTED CLADE

CLADE BW6 (Tip)

TYPE OF DISTANCE

WITHIN CLADE

NESTED CLADE

CLADE BW7 (Tip)

TYPE OF DISTANCE WITHIN CLADE NESTED CLADE

DISTANCE

○. 0000

98.5282

DISTANCE

10.5012

55.9542

$\begin{array}{rrr}\text { DISTANCE } & \text { PROB. }<= & \text { PROB . }>= \\ 26.4809 & 0.7814 & 0.2186 \\ 63.1864 & 0.9418 & 0.0582\end{array}$

$\begin{array}{rrr}\text { DISTANCE } & \text { PROB. }<= & \text { PROB. }>= \\ 0.000 \odot & 0.6580 & 1.0000 \\ 26.3939 & 0.6594 & 0.5610\end{array}$

PROB . $<=$

PROB . $>=$

0.6588

1.0000

$\odot .9774$

๑. 0226

$\begin{array}{rrr}\text { DISTANCE } & \text { PROB. }<= & \text { PROB. }>= \\ 0.000 \odot & 0.6635 & 1.000 \odot \\ 8.7998 & 0.0474 & 0.9526\end{array}$

PROB . $<=$

PROB . > =

0.6395

$\odot .3669$

0.8731

$\odot .1269$

PART III. TEST OF INTERIOR VS. TIP CLADES:

$\begin{array}{rrrr}\text { TYPE OF DISTANCE } & \text { I-T DISTANCE } & \text { PROB. }<= & \text { PROB. }>= \\ \text { WITHIN CLADE } & 18.6760 & 0.0561 & 0.9439 \\ \text { NESTED CLADE } & -22.7815 & 0.0224 & 0.9776\end{array}$

PERMUTATION ANALYSIS OF 1-7 WAITAKI \#2 (5)

PART I. EXACT CONTINGENCY TEST: OBSERVED CHI-SQUARE STATISTIC =

THE PROBABILITY OF A RANDOM CHI-SQUARE BEING GREATER THAN OR EQUAL TO THE OBSERVED CHI-SQUARE = $\odot .3291$ 
PART II. GEOGRAPHIC DISTANCE ANALYSIS:

\begin{tabular}{|c|c|c|c|c|}
\hline \multirow{4}{*}{$\begin{array}{r}\text { CLADE } \\
\text { TYPE }\end{array}$} & \multicolumn{4}{|l|}{ BW3 (Interior) } \\
\hline & OF DISTANCE & DISTANCE & PROB. <= & PROB . $>=$ \\
\hline & WITHIN CLADE & $0.000 \odot$ & 0.3291 & 1.0000 \\
\hline & NESTED CLADE & 21.8437 & 0.3291 & 1.0000 \\
\hline CLADE & BW10 (Tip) & & & \\
\hline TYPE & OF DISTANCE & DISTANCE & PROB . $<=$ & PROB . $>=$ \\
\hline & WITHIN CLADE & $\odot .0 \odot \odot \odot$ & 1.0000 & 1.0000 \\
\hline & NESTED CLADE & 43.6253 & 0.6709 & 0.3291 \\
\hline
\end{tabular}

PART III. TEST OF INTERIOR VS. TIP CLADES:

\begin{tabular}{|c|c|c|c|c|}
\hline TYPE & OF DISTANCE & I-T DISTANCE & PROB. $<=$ & PROB. $>=$ \\
\hline & WITHIN CLADE & 0.0000 & ๑. 3291 & 1.0000 \\
\hline & & -217816 & & 0.6709 \\
\hline
\end{tabular}

PERMUTATION ANALYSIS OF 1-10 MARLBOROUGH (6)

PART I. EXACT CONTINGENCY TEST: OBSERVED CHI-SQUARE STATISTIC $=19.0278$

THE PROBABILITY OF A RANDOM CHI-SQUARE BEING GREATER THAN OR EQUAL TO THE OBSERVED CHI-SQUARE $=\quad 0.5887$

PART II. GEOGRAPHIC DISTANCE ANALYSIS:

\begin{tabular}{|c|c|c|c|c|}
\hline CLADE & BM1 (Interior) & & & \\
\hline TYPE & OF DISTANCE & DISTANCE & PROB . <= & PROB. $>=$ \\
\hline & WITHIN CLADE & 30.3075 & 0.7720 & 0.2280 \\
\hline & NESTED CLADE & 30.1989 & 0.6305 & 0.3695 \\
\hline CLADE & BM2 (Interior) & & & \\
\hline TYPE & OF DISTANCE & DISTANCE & PROB . $<=$ & PROB . $>=$ \\
\hline & WITHIN CLADE & 18.2770 & 0.4245 & 0.5755 \\
\hline & NESTED CLADE & 23.7911 & $\odot .2922$ & 0.7078 \\
\hline CLADE & BM3 (Tip) & & & \\
\hline TYPE & OF DISTANCE & DISTANCE & PROB . $<=$ & PROB. $>=$ \\
\hline & WITHIN CLADE & 9.3997 & 0.2467 & 0.7533 \\
\hline & NESTED CLADE & 13.4961 & 0.1989 & 0.8011 \\
\hline CLADE & BM5 (Tip) & & & \\
\hline TYPE & OF DISTANCE & DISTANCE & PROB . $<=$ & PROB. $>=$ \\
\hline & WITHIN CLADE & 0.0000 & 0.6963 & 1.0000 \\
\hline & NESTED CLADE & 39.7849 & 0.8976 & 0.1024 \\
\hline
\end{tabular}

PART III. TEST OF INTERIOR VS. TIP CLADES:

$\begin{array}{rrrr}\text { TYPE OF DISTANCE } & \text { I-T DISTANCE } & \text { PROB. }<= & \text { PROB. }>= \\ \text { WITHIN CLADE } & 18.8851 & 0.7594 & 0.2406 \\ \text { NESTED CLADE } & 5.1937 & 0.6508 & 0.3492\end{array}$

PERMUTATION ANALYSIS OF 1-16 NORTH ISLAND (7)

PART I. EXACT CONTINGENCY TEST: OBSERVED CHI-SQUARE STATISTIC $=13.7500$ THE PROBABILITY OF A RANDOM CHI-SQUARE BEING GREATER THAN OR EQUAL TO THE OBSERVED CHI-SQUARE $=0.2488$

PART II. GEOGRAPHIC DISTANCE ANALYSIS: 


\begin{tabular}{|c|c|c|c|c|}
\hline CLADE & A1 (Interior) & & & \\
\hline TYPE & OF DISTANCE & DISTANCE & PROB . <= & PROB. $>=$ \\
\hline & WITHIN CLADE & 8.4522 & 0.5151 & 0.4849 \\
\hline & NESTED CLADE & 9.4412 & 0.7270 & 0.2730 \\
\hline CLADE & A2 (Tip) & & & \\
\hline TYPE & OF DISTANCE & DISTANCE & PROB . <= & PROB. $>=$ \\
\hline & WITHIN CLADE & 0.0000 & 1.0000 & 1.0000 \\
\hline & NESTED CLADE & 3.8441 & 0.0000 & 1.0000 \\
\hline CLADE & A3 (Tip) & & & \\
\hline TYPE & OF DISTANCE & DISTANCE & PROB . $<=$ & PROB . $>=$ \\
\hline & WITHIN CLADE & 0.0000 & 1.0000 & 1.0000 \\
\hline & NESTED CLADE & 14.7872 & 0.5417 & 0.4583 \\
\hline CLADE & A4 (Tip) & & & \\
\hline TYPE & OF DISTANCE & DISTANCE & PROB . $<=$ & PROB . $>=$ \\
\hline & WITHIN CLADE & 0.0000 & 1.0000 & 1.0000 \\
\hline & NESTED CLADE & 14.7872 & 0.5481 & $\odot .4519$ \\
\hline
\end{tabular}

PART III. TEST OF INTERIOR VS. TIP CLADES:

$\begin{array}{rrrr}\text { TYPE OF DISTANCE } & \text { I-T DISTANCE } & \text { PROB. }<= & \text { PROB. }>= \\ \text { WITHIN CLADE } & 8.4522 & 0.5151 & 0.4849 \\ \text { NESTED CLADE } & -1.6983 & 0.6378 & 0.3622\end{array}$

PERMUTATION ANALYSIS OF 1-17 NORTH OTAGO (8)

PART I. EXACT CONTINGENCY TEST: OBSERVED CHI-SQUARE STATISTIC $=14.9650$

THE PROBABILITY OF A RANDOM CHI-SQUARE BEING GREATER THAN OR EQUAL TO THE OBSERVED CHI-SQUARE $=\quad 0.4970$

PART II. GEOGRAPHIC DISTANCE ANALYSIS:

\begin{tabular}{|c|c|c|c|c|}
\hline CLADE & CN1 (Interior) & & & \\
\hline TYPE & OF DISTANCE & DISTANCE & PROB . $<=$ & PROB . $>=$ \\
\hline & WITHIN CLADE & 38.6993 & 0.2608 & 0.7392 \\
\hline & NESTED CLADE & 38.8070 & 0.2624 & 0.7376 \\
\hline CLADE & CN2 (Tip) & & & \\
\hline TYPE & OF DISTANCE & DISTANCE & PROB . $<=$ & PROB. $>=$ \\
\hline & WITHIN CLADE & $\odot .00 \odot \odot$ & 0.1979 & $1.000 \odot$ \\
\hline & NESTED CLADE & 56.3321 & 0.7347 & $\odot .2653$ \\
\hline CLADE & CN3 (Tip) & & & \\
\hline TYPE & OF DISTANCE & DISTANCE & PROB . $<=$ & PROB . $>=$ \\
\hline & WITHIN CLADE & 0.0000 & $\odot .9731$ & 1.0000 \\
\hline & NESTED CLADE & 45.1219 & 0.5294 & 0.4706 \\
\hline
\end{tabular}

PART III. TEST OF INTERIOR VS. TIP CLADES:

$\begin{array}{rrrr}\text { TYPE OF DISTANCE } & \text { I-T DISTANCE } & \text { PROB. }<= & \text { PROB. }>= \\ \text { WITHIN CLADE } & 38.6993 & 0.8052 & 0.1948 \\ \text { NESTED CLADE } & -13.7884 & 0.2385 & 0.7615\end{array}$


THE PROBABILITY OF A RANDOM CHI-SQUARE BEING GREATER THAN OR EQUAL TO THE OBSERVED CHI-SQUARE $=\quad 0.1816$

PART II. GEOGRAPHIC DISTANCE ANALYSIS:

CLADE CS1 (Interior)

TYPE OF DISTANCE

WITHIN CLADE

DISTANCE

18. 7031

PROB.<=
$0.000 \odot$
$0.000 \odot$

PROB . > =

NESTED CLADE

20.7631

1. 0000

1.0000

CLADE CS3 (Tip)

TYPE OF DISTANCE WITHIN CLADE NESTED CLADE

$\begin{array}{rrr}\text { DISTANCE } & \text { PROB. }<= & \text { PROB. }>= \\ 0.000 \odot & 0.5605 & 1.000 \odot \\ 48.0342 & 1.0000 & 0.0592\end{array}$

PART III. TEST OF INTERIOR VS. TIP CLADES:

$\begin{array}{rrrr}\text { TYPE OF DISTANCE } & \text { I-T DISTANCE } & \text { PROB. }<= & \text { PROB. }>= \\ \text { WITHIN CLADE } & 18.7031 & 0.00 \odot \odot & 1.00 \odot \odot \\ \text { NESTED CLADE } & -27.2711 & 0.0 \odot \odot \odot & 1.00 \odot \odot\end{array}$

PERMUTATION ANALYSIS OF 1-20 SOUTH OTAGO \#2 (10)

PART I. EXACT CONTINGENCY TEST: OBSERVED CHI-SQUARE STATISTIC =

$\odot .7500$

THE PROBABILITY OF A RANDOM CHI-SQUARE BEING GREATER THAN OR EQUAL TO THE OBSERVED CHI-SQUARE $=1.0000$

PART II. GEOGRAPHIC DISTANCE ANALYSIS:

CLADE CS2 (Interior)

TYPE OF DISTANCE WITHIN CLADE NESTED CLADE

DISTANCE

5. 3569

4.6874

DISTANCE

○. 0000

2. 1871
PROB . $<=$

1.0000

1.0000

PROB . $<=$

1.0000

$\odot .6614$
PROB . > =

○. 0000

$\odot .6614$

PROB . > =

1.0000

1. $\odot \odot \odot \odot$

PART III. TEST OF INTERIOR VS. TIP CLADES:

$\begin{array}{rrrr}\text { TYPE OF DISTANCE } & \text { I-T DISTANCE } & \text { PROB. }<= & \text { PROB. }>= \\ \text { WITHIN CLADE } & 5.3569 & 1.000 \odot & 0.0000 \\ \text { NESTED CLADE } & 2.5003 & 1.000 \odot & 0.6614\end{array}$

PERMUTATION ANALYSIS OF 2-1 SOUTHERN ALPS A (11)

PART I. EXACT CONTINGENCY TEST: OBSERVED CHI-SQUARE STATISTIC =

9.9107

THE PROBABILITY OF A RANDOM CHI-SQUARE BEING GREATER THAN OR EQUAL TO THE OBSERVED CHI-SQUARE $=0.6293$

PART II. GEOGRAPHIC DISTANCE ANALYSIS:

CLADE 1-1 (Interior)

TYPE OF DISTANCE

WITHIN CLADE

NESTED CLADE

$\begin{array}{rrr}\text { DISTANCE } & \text { PROB . }<= & \text { PROB . }>= \\ 49.8765 & 0.1603 & 0.8397 \\ 49.7641 & 0.0409 & 0.9591\end{array}$


CLADE 1-2 (Tip)

TYPE OF DISTANCE WITHIN CLADE

DISTANCE

2.3863

69.9223
PROB . $<=$

$$
\odot .0971
$$

$\odot .8733$
PROB . > =

0.9217

$\odot .1455$

PART III. TEST OF INTERIOR VS. TIP CLADES:

TYPE OF DISTANCE WITHIN CLADE NESTED CLADE

$\begin{array}{rr}\text { I - T } & \text { DISTANCE } \\ & 47.4901 \\ -20.1582\end{array}$

PROB . $<=$
0.9029
0.1455

PROB . > =

$\odot .0971$

$\odot .8545$

PERMUTATION ANALYSIS OF 2-3 SOUTHERN ALPS C (12)

PART I. EXACT CONTINGENCY TEST: OBSERVED CHI-SQUARE STATISTIC =

THE PROBABILITY OF A RANDOM CHI-SQUARE BEING GREATER THAN OR EQUAL TO THE OBSERVED CHI-SQUARE $=\quad 0.5151$

PART II. GEOGRAPHIC DISTANCE ANALYSIS:

CLADE 1-4 (Interior)

TYPE OF DISTANCE WITHIN CLADE

DISTANCE

68.0736 NESTED CLADE

CLADE 1-5 (Tip)

TYPE OF DISTANCE WITHIN CLADE NESTED CLADE
69.1575

DISTANCE

○. 0000

109.3031
PROB . $<=$

0.1175

$\odot .0673$

PROB . $>=$

$\odot .8825$

$\odot .9327$

$\begin{array}{rr}\text { PROB. }<= & \text { PROB. }>= \\ 0.1617 & 1.0000 \\ 0.9327 & 0.0673\end{array}$

PART III. TEST OF INTERIOR VS. TIP CLADES:

$\begin{array}{rrrr}\text { TYPE OF DISTANCE } & \text { I-T DISTANCE } & \text { PROB. }<= & \text { PROB. }>= \\ \text { WITHIN CLADE } & 68.0736 & 0.8569 & 0.1431 \\ \text { NESTED CLADE } & -40.1456 & 0.0673 & 0.9327\end{array}$

PERMUTATION ANALYSIS OF 2-4 WAITAKI (13)

PART I. EXACT CONTINGENCY TEST: OBSERVED CHI-SQUARE STATISTIC $=95.3220$

THE PROBABILITY OF A RANDOM CHI-SQUARE BEING GREATER THAN OR EQUAL TO THE OBSERVED CHI-SQUARE $=\quad 0.0504$

PART II. GEOGRAPHIC DISTANCE ANALYSIS:

\begin{tabular}{|c|c|c|c|c|}
\hline CLADE & 1-6 (Interior) & & & \\
\hline TYPE & OF DISTANCE & DISTANCE & PROB . $<=$ & PROB. $>=$ \\
\hline & WITHIN CLADE & 37.0400 & 0.0093 & ๑. 9907 \\
\hline & NESTED CLADE & 39.9541 & $\odot .0409$ & ○. 9591 \\
\hline CLADE & 1-7 (Tip) & & & \\
\hline TYPE & OF DISTANCE & DISTANCE & PROB . $<=$ & PROB. $>=$ \\
\hline & WITHIN CLADE & 29.1043 & $\odot .6810$ & ๑. 3190 \\
\hline & NESTED CLADE & 36.2873 & $\odot .4277$ & 0.5723 \\
\hline CLADE & 1-8 (Tip) & & & \\
\hline TYPE & OF DISTANCE & DISTANCE & PROB . $<=$ & PROB. $>=$ \\
\hline & WITHIN CLADE & $\odot . \odot \odot \odot \odot$ & $\odot .6986$ & $1.0 \odot \odot \odot$ \\
\hline & NESTED CLADE & 75.8706 & 0.9625 & 0.1849 \\
\hline CLADE & 1-9 (Tip) & & & \\
\hline TYPE & OF DISTANCE & DISTANCE & PROB . $<=$ & PROB. $>=$ \\
\hline & WITHIN CLADE & 0.0000 & 0.6980 & 1.0000 \\
\hline
\end{tabular}



NESTED CLADE
147.2767
1. 0000
0.0184

PART III. TEST OF INTERIOR VS. TIP CLADES:

$\begin{array}{rrrr}\text { TYPE OF DISTANCE } & \text { I-T } \text { DISTANCE } & \text { PROB. }<= & \text { PROB. }>= \\ \text { WITHIN CLADE } & 19.5774 & 0.0966 & 0.9034 \\ \text { NESTED CLADE } & -26.4478 & 0.0709 & 0.9291\end{array}$

PERMUTATION ANALYSIS OF 2-5 MARLBOROUGH + NELSON (14)

PART I. EXACT CONTINGENCY TEST: OBSERVED CHI-SQUARE STATISTIC = 29.1833

THE PROBABILITY OF A RANDOM CHI-SQUARE BEING GREATER THAN OR EQUAL TO THE OBSERVED CHI-SQUARE $=\quad 0.1868$

PART II. GEOGRAPHIC DISTANCE ANALYSIS:

\begin{tabular}{|c|c|c|c|c|}
\hline CLADE & 1-10 (Interior) & & & \\
\hline TYPE & OF DISTANCE & DISTANCE & PROB . $<=$ & PROB. $>=$ \\
\hline & WITHIN CLADE & 28.0427 & 0.1254 & 0.8746 \\
\hline & NESTED CLADE & 27.9774 & 0.1116 & ๑.8884 \\
\hline CLADE & 1-11 (Tip) & & & \\
\hline TYPE & OF DISTANCE & DISTANCE & PROB . $<=$ & PROB . $>=$ \\
\hline & WITHIN CLADE & 0.0000 & 0.8251 & $1.00 \odot \odot$ \\
\hline & NESTED CLADE & 45.2513 & 0.5889 & $\odot .5297$ \\
\hline CLADE & 1-12 (Tip) & & & \\
\hline TYPE & OF DISTANCE & DISTANCE & PROB. $<=$ & PROB. $>=$ \\
\hline & WITHIN CLADE & 0.0000 & 0.8305 & 1.0000 \\
\hline & NESTED CLADE & 3.1475 & 0.1860 & 0.8140 \\
\hline CLADE & 1-13 (Tip) & & & \\
\hline TYPE & OF DISTANCE & DISTANCE & PROB . $<=$ & PROB. $>=$ \\
\hline & WITHIN CLADE & $0.000 \odot$ & 0.0001 & 1.0000 \\
\hline & NESTED CLADE & 75.4033 & 1.0000 & 0.0001 \\
\hline
\end{tabular}

PART III. TEST OF INTERIOR VS. TIP CLADES:

$\begin{array}{rrrr}\text { TYPE OF DISTANCE } & \text { I-T } \text { DISTANCE } & \text { PROB. }<= & \text { PROB. }>= \\ \text { WITHIN CLADE } & 28.0427 & 0.9561 & 0.0439 \\ \text { NESTED CLADE } & -32.7962 & 0.0027 & 0.9973\end{array}$

PERMUTATION ANALYSIS OF 2-6 KAIKOURA (15)

PART I. EXACT CONTINGENCY TEST: OBSERVED CHI-SQUARE STATISTIC $=\quad 2.0000$

THE PROBABILITY OF A RANDOM CHI-SQUARE BEING GREATER THAN OR EQUAL TO THE OBSERVED CHI-SQUARE $=\quad 1.00 \odot \odot$

PART II. GEOGRAPHIC DISTANCE ANALYSIS:

\begin{tabular}{|c|c|c|c|c|}
\hline \multirow{2}{*}{$\begin{array}{r}\text { CLADE } \\
\text { TYPE }\end{array}$} & \multicolumn{4}{|l|}{ 1-14 (Tip) } \\
\hline & OF DISTANCE & DISTANCE & PROB . $<=$ & PROB. $>=$ \\
\hline & WITHIN CLADE & ๑. .०००० & $1.0 \odot \odot \odot$ & $1.000 \odot$ \\
\hline & NESTED CLADE & 14.0352 & 0.5024 & 0.4976 \\
\hline CLADE & 1-15 (Interior) & & & \\
\hline TYPE & OF DISTANCE & DISTANCE & PROB . <= & PROB . $>=$ \\
\hline & WITHIN CLADE & 0.0000 & 1.0000 & 1.0000 \\
\hline & NESTED CLADE & 14.0341 & 0.4976 & 1.0000 \\
\hline
\end{tabular}

PART III. TEST OF INTERIOR VS. TIP CLADES: 


$\begin{array}{lrrr}\text { TYPE OF DISTANCE } & \text { I-T DISTANCE } & \text { PROB. }<= & \text { PROB. }>= \\ \text { WITHIN CLADE } & 0.0000 & 1.0000 & 1.0000 \\ \text { NESTED CLADE } & -0.0011 & 0.4976 & 0.5024\end{array}$

\section{PERMUTATION ANALYSIS OF 2-8 NORTH OTAGO (16)}

PART I. EXACT CONTINGENCY TEST: OBSERVED CHI-SQUARE STATISTIC $=\mathbf{8 . 4 7 9 2}$

THE PROBABILITY OF A RANDOM CHI-SQUARE BEING GREATER THAN OR EQUAL TO THE OBSERVED CHI-SQUARE $=0.3991$

PART II. GEOGRAPHIC DISTANCE ANALYSIS:

$\begin{array}{rlrr}\text { CLADE } & 1-17 \text { (Interior) } & & \\ \text { TYPE OF DISTANCE } & \text { DISTANCE } & \text { PROB. }<= & \text { PROB. }>= \\ \text { WITHIN CLADE } & 39.2240 & 0.1099 & 0.8901 \\ \text { NESTED CLADE } & 39.4145 & 0.1099 & 0.8901 \\ & & & \\ \text { CLADE } 1-18 \text { (Tip) } & & & \\ \text { TYPE OF DISTANCE } & \text { DISTANCE } & \text { PROB. }<= & \text { PROB. }>= \\ \text { WITHIN CLADE } & 0.0000 & 0.9722 & 1.0000 \\ \text { NESTED CLADE } & 43.6531 & 0.5376 & 0.5705\end{array}$

PART III. TEST OF INTERIOR VS. TIP CLADES:

$\begin{array}{lrrr}\text { TYPE OF DISTANCE } & \text { I-T DISTANCE } & \text { PROB. }<= & \text { PROB. }>= \\ \text { WITHIN CLADE } & 39.2240 & 0.1099 & 0.8901 \\ \text { NESTED CLADE } & -4.2386 & 0.4624 & 0.5376\end{array}$

\section{PERMUTATION ANALYSIS OF 2-9 SOUTH OTAGO (17)}

PART I. EXACT CONTINGENCY TEST: OBSERVED CHI-SQUARE STATISTIC $=12.9833$

THE PROBABILITY OF A RANDOM CHI-SQUARE BEING GREATER THAN OR EQUAL TO THE OBSERVED CHI-SQUARE $=0.0447$

PART II. GEOGRAPHIC DISTANCE ANALYSIS:

\begin{tabular}{|c|c|c|c|c|}
\hline \multirow{3}{*}{$\begin{array}{l}\text { CLADE } \\
\text { TYPE }\end{array}$} & $\begin{array}{l}\text { 1-19 ( Interl } \\
\text { OF DISTANCE }\end{array}$ & & & \\
\hline & WITHIN CLADE & 24.3752 & 0.0695 & 0.9305 \\
\hline & NESTED CLADE & 26.8019 & 0.1886 & 0.8114 \\
\hline \multirow{4}{*}{$\begin{array}{r}\text { CLADE } \\
\text { TYPE }\end{array}$} & 1-20 (Tip) & & & \\
\hline & OF DISTANCE & DISTANCE & PROB . $<=$ & PROB . $>=$ \\
\hline & WITHIN CLADE & 3.6456 & 0.0601 & 0.9465 \\
\hline & NESTED CLADE & 30.0101 & 0.8175 & 0.1825 \\
\hline
\end{tabular}

PART III. TEST OF INTERIOR VS. TIP CLADES:

$\begin{array}{lrrr}\text { TYPE OF DISTANCE } & \text { I-T DISTANCE } & \text { PROB. }<= & \text { PROB. }>= \\ \text { WITHIN CLADE } & 20.7296 & 0.8951 & 0.1049 \\ \text { NESTED CLADE } & -3.2082 & 0.1825 & 0.8175\end{array}$

PERMUTATION ANALYSIS OF 3-1 SOUTHERN ALPS + WAITAKI (18)

PART I. EXACT CONTINGENCY TEST: OBSERVED CHI-SQUARE STATISTIC $=380.2256$ THE PROBABILITY OF A RANDOM CHI-SQUARE BEING GREATER THAN 
OR EQUAL TO THE OBSERVED CHI-SQUARE =

$\odot .0000$

PART II. GEOGRAPHIC DISTANCE ANALYSIS:

\begin{tabular}{|c|c|c|c|c|}
\hline CLADE & 2-1 (Interior & & & \\
\hline TYPE & OF DISTANCE & DISTANCE & PROB. $<=$ & PROB. $>=$ \\
\hline & WITHIN CLADE & 50.9082 & $\odot .0 \odot \odot \odot$ & 1.0000 \\
\hline & NESTED CLADE & 210.4752 & 1.0000 & 0.0000 \\
\hline CLADE & 2-2 (Tip) & & & \\
\hline TYPE & OF DISTANCE & DISTANCE & PROB . $<=$ & PROB. $>=$ \\
\hline & WITHIN CLADE & 39.1682 & $0.00 \odot \odot$ & 1.0000 \\
\hline & NESTED CLADE & 175.3378 & 0.9940 & 0.0060 \\
\hline CLADE & 2-3 (Tip) & & & \\
\hline TYPE & OF DISTANCE & DISTANCE & PROB. <= & PROB. $>=$ \\
\hline & WITHIN CLADE & 88.4700 & 0.0183 & 0.9817 \\
\hline & NESTED CLADE & 144.9838 & 0.6955 & 0.3045 \\
\hline CLADE & 2-4 (Tip) & & & \\
\hline TYPE & OF DISTANCE & DISTANCE & PROB . <= & PROB. $>=$ \\
\hline & WITHIN CLADE & 46.6660 & 0.0000 & 1.0000 \\
\hline & NESTED CLADE & 48.9781 & $\odot . \odot \odot \odot \odot$ & 1.0000 \\
\hline
\end{tabular}

PART III. TEST OF INTERIOR VS. TIP CLADES:

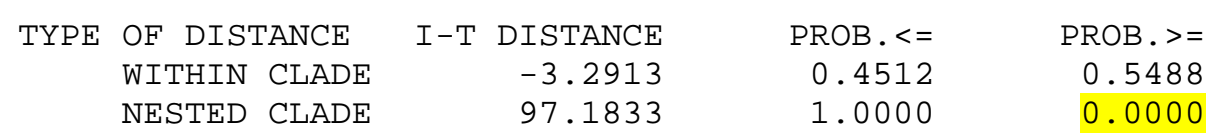

PERMUTATION ANALYSIS OF 3-2 NMK (19)

PART I. EXACT CONTINGENCY TEST: OBSERVED CHI-SQUARE STATISTIC $=19.0000$

THE PROBABILITY OF A RANDOM CHI-SQUARE BEING GREATER THAN OR EQUAL TO THE OBSERVED CHI-SQUARE $=0.0659$

PART II. GEOGRAPHIC DISTANCE ANALYSIS:

$\begin{array}{rlrr}\text { CLADE } & 2-5 \text { (Interior) } & & \\ \text { TYPE OF DISTANCE } & \text { DISTANCE } & \text { PROB. }<= & \text { PROB. }>= \\ \text { WITHIN CLADE } & 33.9507 & 0.0065 & 0.9935 \\ \text { NESTED CLADE } & 38.2908 & 0.0000 & 1.0000 \\ & & & \\ \text { CLADE } 2-6 \text { (Tip) } & & & \\ \text { TYPE OF DISTANCE } & \text { DISTANCE } & \text { PROB.<= } & \text { PROB. >= } \\ \text { WITHIN CLADE } & 14.0347 & 0.2669 & 0.7396 \\ \text { NESTED CLADE } & 72.9158 & 0.8807 & 0.1193\end{array}$

PART III. TEST OF INTERIOR VS. TIP CLADES:

$\begin{array}{rrrr}\text { TYPE OF DISTANCE } & \text { I-T DISTANCE } & \text { PROB. }<= & \text { PROB. }>= \\ \text { WITHIN CLADE } & 19.9160 & 0.5667 & 0.4333 \\ \text { NESTED CLADE } & -34.6250 & 0.0840 & 0.9160\end{array}$

\section{PERMUTATION ANALYSIS OF 3-4 OTAGO (20)}

PART I. EXACT CONTINGENCY TEST: OBSERVED CHI-SQUARE STATISTIC = 44.4889

THE PROBABILITY OF A RANDOM CHI-SQUARE BEING GREATER THAN OR EQUAL TO THE OBSERVED CHI-SQUARE $=\quad 0.00 \odot \odot$ 
PART II. GEOGRAPHIC DISTANCE ANALYSIS:

\begin{tabular}{|c|c|c|c|c|}
\hline \multirow{4}{*}{$\begin{array}{r}\text { CLADE } \\
\text { TYPE }\end{array}$} & \multicolumn{4}{|l|}{ 2-8 (Interior) } \\
\hline & OF DISTANCE & DISTANCE & PROB . $<=$ & PROB . > = \\
\hline & WITHIN CLADE & 39.5523 & $\odot . \odot \odot \odot \odot$ & 1.0000 \\
\hline & NESTED CLADE & 48.2555 & 0.0703 & $\odot .9297$ \\
\hline \multirow{4}{*}{$\begin{array}{r}\text { CLADE } \\
\text { TYPE }\end{array}$} & 2-9 (Interior) & & & \\
\hline & OF DISTANCE & DISTANCE & PROB . $<=$ & PROB. $>=$ \\
\hline & WITHIN CLADE & 28.4126 & 0.0023 & 0.9977 \\
\hline & NESTED CLADE & 59.1841 & 0.8264 & 0.1736 \\
\hline
\end{tabular}

NO INTERIOR/TIP CLADES EXIST IN THIS GROUP

PERMUTATION ANALYSIS OF 4-1 SOUTH ISLAND (NOT OTAGO) (21)

PART I. EXACT CONTINGENCY TEST: OBSERVED CHI-SQUARE STATISTIC $=148.0000$

THE PROBABILITY OF A RANDOM CHI-SQUARE BEING GREATER THAN OR EQUAL TO THE OBSERVED CHI-SQUARE $=\quad 0.000 \odot$

PART II. GEOGRAPHIC DISTANCE ANALYSIS:

\begin{tabular}{|c|c|c|c|c|}
\hline \multirow{4}{*}{$\begin{array}{r}\text { CLADE } \\
\text { TYPE }\end{array}$} & \multicolumn{4}{|l|}{ 3-1 (Interior) } \\
\hline & OF DISTANCE & DISTANCE & PROB . $<=$ & PROB. $>=$ \\
\hline & WITHIN CLADE & 134.4319 & 0.0000 & $1.0 \odot \odot \odot$ \\
\hline & NESTED CLADE & 150.6586 & 0.0000 & 1.0000 \\
\hline \multicolumn{5}{|l|}{ CLADE } \\
\hline TYPE & OF DISTANCE & DISTANCE & PROB . <= & PROB . $>=$ \\
\hline & WITHIN CLADE & 45.2158 & $\odot .0 \odot \odot \odot$ & $1.0 \odot \odot \odot$ \\
\hline & NESTED CLADE & 269.0434 & 1.0000 & 0.0000 \\
\hline
\end{tabular}

NO INTERIOR/TIP CLADES EXIST IN THIS GROUP 


\section{M. campbelli Geodis Total-mtDNA input:}

26

1-1 SOUTHERN ALPS A\&C1 (1)

11

BSA1bsa1 BSA1bsa2 BSA1bsa3 BSA1bsa4 BSA1bsa5 BSA2bsa1 BSA3bsa1 BSA4bsa1 BSA5bsa1
$\odot$
1
1
1
1
1
1
1

BSA8bsa1 BSC1bsa1

17

$\begin{array}{lllllllllllllllll}12 & 15 & 16 & 17 & 20 & 21 & 22 & 23 & 24 & 25 & 42 & 43 & 44 & 45 & 48 & 64 & 69\end{array}$

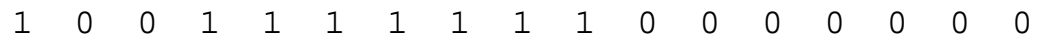

$\begin{array}{lllllllllllllllll}0 & 0 & 2 & 0 & 0 & 0 & 0 & 0 & 0 & 0 & 0 & 0 & 0 & 0 & 0 & 0 & 0\end{array}$

$\begin{array}{lllllllllllllllll}0 & 0 & 0 & 0 & 0 & 0 & 0 & 0 & 0 & 0 & 0 & 0 & 1 & 0 & 0 & 0 & 0\end{array}$

$\begin{array}{lllllllllllllllll}1 & \odot & \odot & \odot & \odot & \odot & \odot & \odot & \odot & \odot & \odot & \odot & \odot & \odot & \odot & \odot & 0\end{array}$

$\begin{array}{lllllllllllllllll}0 & 1 & 0 & 0 & 0 & 0 & 0 & 0 & 0 & 0 & 0 & 0 & 0 & 0 & 0 & 0 & 0\end{array}$

$\odot \begin{array}{lllllllllllllllll}\odot & 0 & 0 & 0 & 0 & 0 & 0 & 0 & 1 & 0 & 1 & 0 & 0 & 1 & 0 & 0 & 0\end{array}$

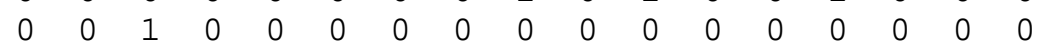

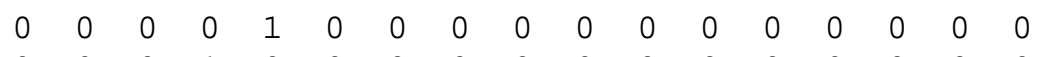

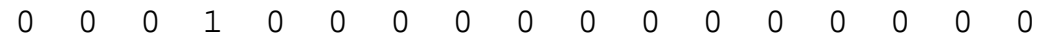

$\odot \begin{array}{lllllllllllllllll}\odot & \odot & \odot & \odot & 1 & \odot & \odot & \odot & \odot & \odot & \odot & \odot & \odot & \odot & \odot & \odot & \odot\end{array}$

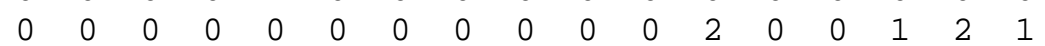

1-4 SOUTHERN ALPS C 1

2

BSC2bsa1 BSC2bsa8

$$
\odot \quad 1
$$

3

$46 \quad 47 \quad 66$

$\begin{array}{lll}1 & 0 & 3\end{array}$

$\odot \quad 10$

2-1 SOUTHERN ALPS A\&C2

5

1-1 1-3 1-4 1-5 1-6 1-7

$\begin{array}{llllll}0 & 1 & 1 & 1 & 1 & 1\end{array}$

21

$\begin{array}{lllllllllllllllllllll}8 & 12 & 15 & 16 & 17 & 20 & 21 & 22 & 23 & 24 & 25 & 42 & 43 & 44 & 45 & 46 & 47 & 48 & 64 & 66 & 69\end{array}$

$\begin{array}{lllllllllllllllllllll}0 & 2 & 1 & 3 & 2 & 3 & 1 & 1 & 1 & 2 & 1 & 1 & 2 & 1 & 1 & 0 & 0 & 1 & 2 & 0 & 1\end{array}$

$\begin{array}{llllllllllllllllllllll}1 & 0 & 0 & 0 & 0 & 0 & 0 & 0 & 0 & 0 & 0 & 0 & 0 & 0 & 0 & 0 & 0 & 0 & 0 & 0 & 0 \\ 0 & 0 & 0 & 2 & 0 & 0 & 0 & 0 & 0 & 0 & 0 & 0 & 0 & 0 & 0 & 1 & 1 & 0 & 0 & 3 & 0\end{array}$

$\begin{array}{lllllllllllllllllllll}0 & 0 & 0 & 2 & 0 & 0 & 0 & 0 & 0 & 0 & 0 & 0 & 0 & 0 & 0 & 1 & 1 & 0 & 0 & 3 & 0 \\ 0 & 0 & 0 & 0 & 0 & 0 & 0 & 0 & 0 & 0 & 0 & 0 & 2 & 0 & 0 & 0 & 0 & 0 & 0 & 0 & 0\end{array}$

$\begin{array}{lllllllllllllllllllll} & 0 & 0 & \odot & 0 & 0 & 0 & 0 & 0 & 0 & 0 & 0 & 0 & 0 & 0 & 0 & 0 & 0 & 1 & 0 & 0\end{array}$

$\begin{array}{lllllllllllllllllllll}\odot & \odot & \odot & 0 & 0 & 0 & 0 & 0 & 0 & 0 & 0 & 0 & 1 & 0 & 0 & 0 & 0 & 0 & 0 & 0 & 0\end{array}$

3-1 SOUTHERN ALPS a\&c3 (3)

2

2-1 $2-2$

$0 \quad 1$

21

$\begin{array}{lllllllllllllllllllll}8 & 12 & 15 & 16 & 17 & 20 & 21 & 22 & 23 & 24 & 25 & 42 & 43 & 44 & 45 & 46 & 47 & 48 & 64 & 66 & 69\end{array}$

$\begin{array}{lllllllllllllllllllll}1 & 2 & 1 & 5 & 2 & 3 & 1 & 1 & 1 & 2 & 1 & 1 & 5 & 1 & 1 & 1 & 1 & 1 & 3 & 3 & 1 \\ 1 & 1 & 0 & 0 & 0 & 0 & 0 & 0 & 0 & 0 & 0 & 0 & 0 & 0 & 0 & 0 & 0 & 0 & 0 & 0 & 0\end{array}$

1-8 SOUTHERN ALPS B1 (4)

6

BSB1bsb1 BSB1bsb2 BSB1bsb3 BSB2bsb1 BSB3bsb1 BSB4bsb1

8

$20 \quad 57 \quad 58 \quad 596061 \quad 64 \quad 65$

$\odot \begin{array}{lllllllll}\odot & 2 & 1 & 1 & 1 & 1 & \odot & 4\end{array}$

$\odot \begin{array}{ccccccccc}\odot & \odot & \odot & \odot & \odot & \odot & 1 & \odot\end{array}$

$\begin{array}{llllllll}1 & 0 & 0 & 0 & 0 & 0 & 0 & 0\end{array}$

$\begin{array}{lllllllllllll}\odot & \odot & \odot & 1 & \odot & \odot & \odot & 0\end{array}$

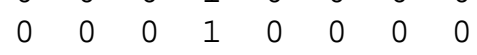

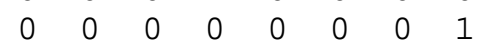

3-2 SOUTHERN ALPS B2 (5)

2

$2-3 \quad 2-4$ 


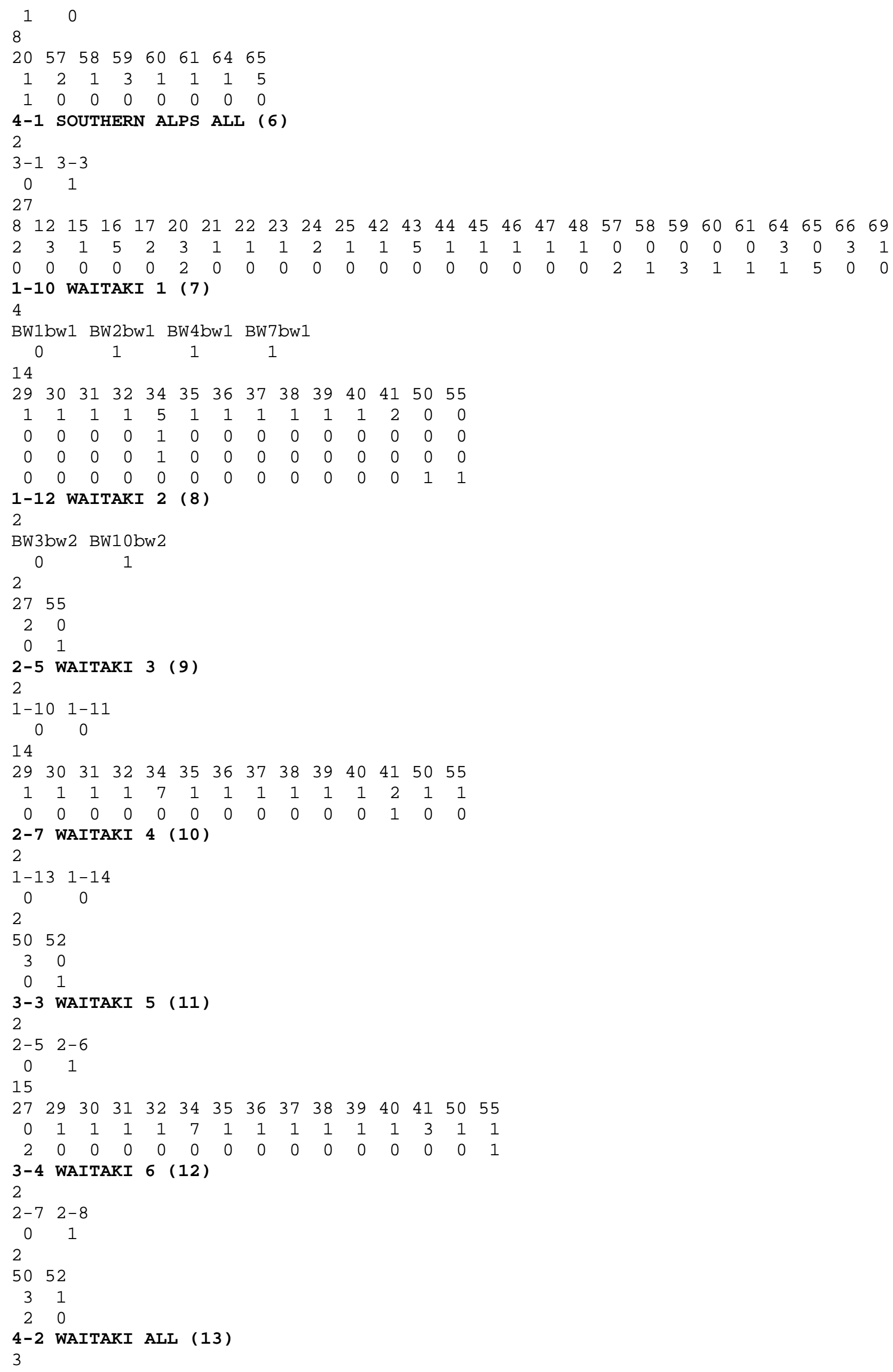




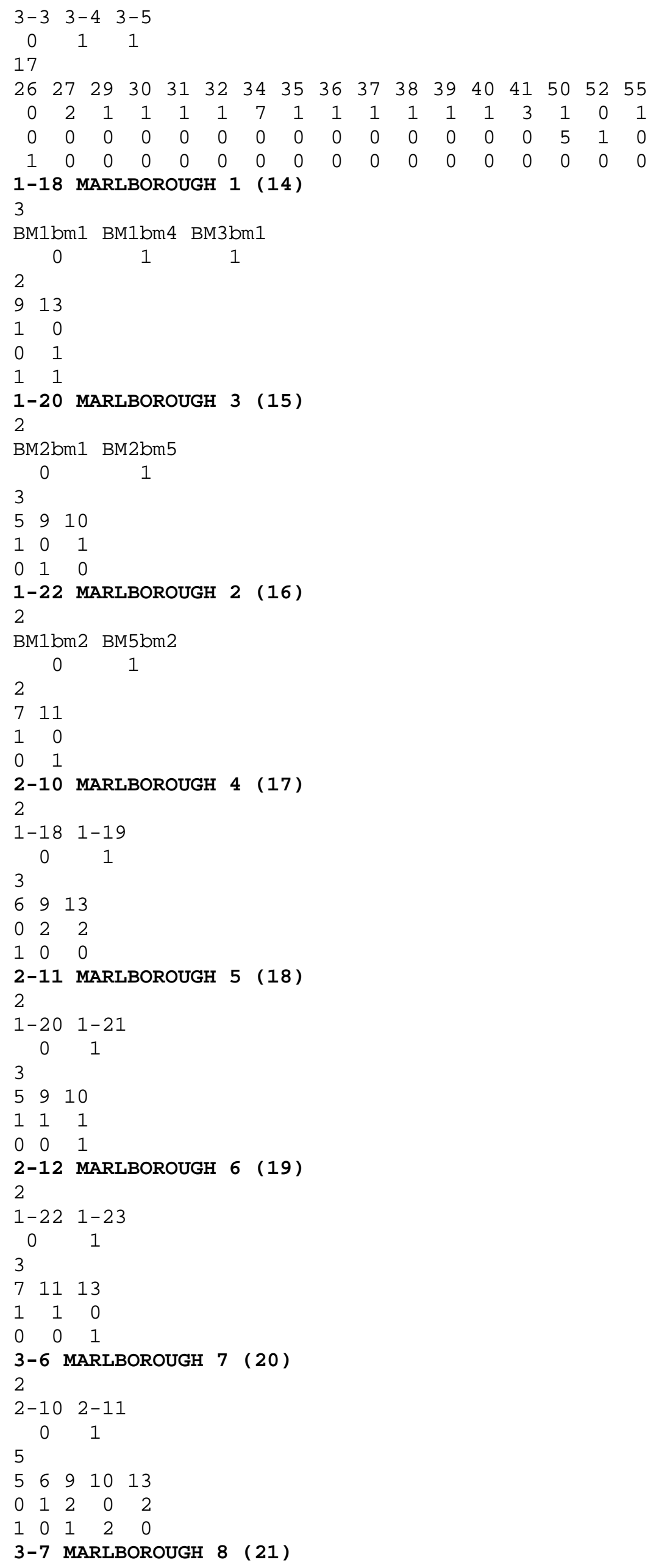


2

2-12 2-13

01

4

$\begin{array}{llll}7 & 11 & 13 & 14\end{array}$

$\begin{array}{llll}1 & 1 & 1 & 0\end{array}$

0005

3-8 KAIKOURA (22)

2

2-14 2-15

10

2

1819

10

○ 1

4-4 MARLBOROUGH 7 + NELSON + KAIKOURA (23)

2

$3-7 \quad 3-8$

$\odot 1$

6

$\begin{array}{llllll}7 & 11 & 13 & 14 & 18 & 19\end{array}$

$\begin{array}{llllll}1 & 1 & 1 & 5 & 1 & 0\end{array}$

$\odot \begin{array}{llllll}0 & 0 & 0 & 0 & 0 & 1\end{array}$

5-1 ALL SOUTH ISLAND (24)

4

4-1 4-2 4-3 4-4

$\begin{array}{llll}1 & 1 & 0 & 1\end{array}$

53

$\begin{array}{llllllllllllllllllllllllllll}5 & 6 & 7 & 9 & 10 & 11 & 12 & 13 & 14 & 15 & 16 & 17 & 18 & 19 & 20 & 21 & 22 & 23 & 24 & 25 & 26 & 27 & 29 & 30 & 31 & 32 & 34 & 35\end{array}$

$\odot \begin{array}{llllllllllllllllllllllllllll}\odot & \odot & \odot & \odot & \odot & 3 & \odot & \odot & 1 & 5 & 2 & \odot & \odot & 5 & 1 & 1 & 1 & 2 & 1 & \odot & \odot & \odot & \odot & \odot & 0 & 0 & 0\end{array}$

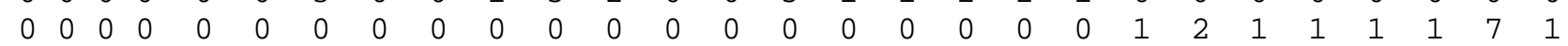

$\begin{array}{lllllllllllllllllllllllllllll}1 & 1 & 0 & 3 & 2 & \odot & 0 & 2 & \odot & \odot & 0 & 0 & 0 & 0 & 0 & 0 & 0 & 0 & 0 & \odot & 0 & 0 & 0 & 0 & 0 & 0 & 0 & 0\end{array}$

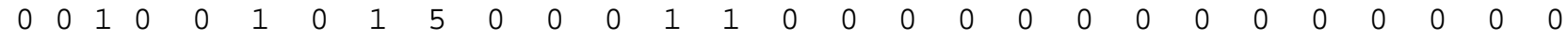

$\begin{array}{lllllllllllllllllllllllll}36 & 37 & 38 & 39 & 40 & 41 & 42 & 43 & 44 & 45 & 46 & 47 & 48 & 50 & 52 & 55 & 57 & 58 & 59 & 60 & 61 & 64 & 65 & 66 & 69\end{array}$

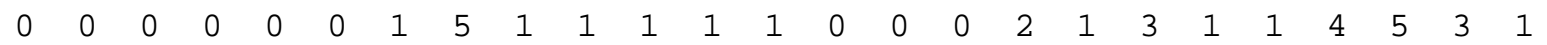

$\begin{array}{llllllllllllllllllllllllllll}1 & 1 & 1 & 1 & 1 & 3 & 0 & 0 & 0 & 0 & 0 & 0 & 0 & 6 & 1 & 1 & 0 & 0 & 0 & 0 & 0 & 0 & 0 & 0 & 0 \\ 0 & 0 & 0 & 0 & 0 & 0 & 0 & 0 & 0 & 0 & 0 & 0 & 0 & 0 & 0 & 0 & 0 & 0 & 0 & 0 & 0 & 0 & 0 & 0 & 0\end{array}$

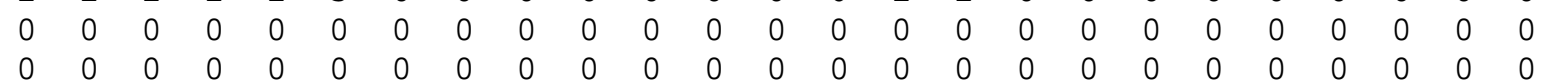

1-28 NORTH ISLAND 1

3

A1a1 A2a1 A3a1

$\begin{array}{lll}0 & 1 & 1\end{array}$

4

$\begin{array}{llll}1 & 2 & 3 & 4\end{array}$

$\begin{array}{llll}1 & 1 & 1 & 0\end{array}$

$\begin{array}{llll}0 & 0 & 0 & 1\end{array}$

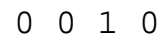

2-16 NORTH ISLAND ALL

2

1-28 1-29

11

$\begin{array}{llll}1 & 2 & 3 & 4\end{array}$

$\begin{array}{llll}1 & 1 & 2 & 1\end{array}$

$\odot \begin{array}{cccc} & 0 & 1 & 0\end{array}$

1-30 OTAGO NORTH 1

3

CN1cn1 CN1cn3 CN2cn1

$\begin{array}{lll}0 & 1 & 1\end{array}$

6

$\begin{array}{llllll}28 & 33 & 34 & 40 & 53 & 54\end{array}$

$\begin{array}{llllll}6 & 4 & 4 & 1 & 1 & 1\end{array}$

$\begin{array}{llllll}0 & 0 & 2 & 0 & 0 & 0\end{array}$

$\begin{array}{llllll}0 & 0 & 2 & 0 & 0 & 0\end{array}$

1-31 OTAGO NORTH 2 
2

CN1cn2 CN4cn2

० 1

3

$\begin{array}{lll}49 & 51 & 67\end{array}$

$\begin{array}{lll}1 & 0 & 1\end{array}$

1-34 OTAGO SOUTH 1

2

CS1cs1 CS3cs1

$0 \quad 1$

6

$\begin{array}{llllll}54 & 56 & 57 & 58 & 63 & 70\end{array}$

$\begin{array}{llllll}0 & 2 & 1 & 1 & 2 & 4\end{array}$

$\begin{array}{llllll}1 & \odot & \odot & \odot & \odot & 0\end{array}$

1-36 OTAGO SOUTH 2

2

CS2cs 2 cs4cs 2

$\begin{array}{ll}0 & 1\end{array}$

2

6263

11

10

2-17 OTAGO 3

3

1-30 1-33 1-34

$\odot \quad \odot \quad 0$

11

$\begin{array}{lllllllllll}28 & 33 & 34 & 40 & 53 & 54 & 56 & 57 & 58 & 63 & 70\end{array}$

$\begin{array}{lllllllllll}6 & 4 & 8 & 1 & 1 & 1 & \odot & 0 & \odot & 0 & 0\end{array}$

$\odot \begin{array}{lllllllllll}\odot & \odot & 0 & \odot & 0 & 0 & \odot & 0 & 0 & 0 & 1\end{array}$

$\begin{array}{lllllllllll}\odot & \odot & \odot & \odot & \odot & 1 & 2 & 1 & 1 & 2 & 4\end{array}$

2-18 OTAGO 4

2

1-31 1-32

01

3

$4951 \quad 67$

$\begin{array}{lll}1 & 1 & 1\end{array}$

○ 10

2-19 OTAGO 5

2

1-35 1-36

$0 \quad 1$

4

$\begin{array}{llll}62 & 63 \quad 66 & 68\end{array}$

$\begin{array}{llll}\odot & 0 & 1 & 1\end{array}$

2100

3-10 OTAGO ALL

3

2-17 2-18 2-19

17

$\begin{array}{lllllllllllllllll}28 & 33 & 34 & 40 & 49 & 51 & 53 & 54 & 56 & 57 & 58 & 62 & 63 & 66 & 67 & 68 & 70\end{array}$

$\begin{array}{lllllllllllllllll}6 & 4 & 8 & 1 & \odot & \odot & 1 & 2 & 2 & 1 & 1 & \odot & 2 & \odot & \odot & \odot & 5\end{array}$

$\odot \begin{array}{lllllllllllllllll} & 0 & 0 & 0 & 1 & 2 & \odot & 0 & 0 & 0 & 0 & 0 & 0 & 0 & 1 & 0 & 0\end{array}$

$\begin{array}{lllllllllllllllll}\odot & \odot & \odot & \odot & \odot & \odot & 0 & \odot & \odot & 0 & 0 & 2 & 1 & 1 & 0 & 1 & 0\end{array}$

END 


\section{M. campbelli Geodis Total-mtDNA output results:}

\section{PERMUTATION ANALYSIS OF 1-1 SOUTHERN ALPS A\&C1 (1)}

PART I. EXACT CONTINGENCY TEST: OBSERVED CHI-SQUARE STATISTIC $=161.9583$

THE PROBABILITY OF A RANDOM CHI-SQUARE BEING GREATER THAN OR EQUAL TO THE OBSERVED CHI-SQUARE $=\quad 0.6018$

PART II. GEOGRAPHIC DISTANCE ANALYSIS:

CLADE BSA1bsa1 (Interior)

TYPE OF DISTANCE WITHIN CLADE NESTED CLADE

CLADE BSA1bsa2 (Tip)

TYPE OF DISTANCE WITHIN CLADE NESTED CLADE

CLADE BSA1bsa3 (Tip)

TYPE OF DISTANCE WITHIN CLADE NESTED CLADE

TYPE OF DISTANCE WITHIN CLADE NESTED CLADE

CLADE BSA1bsa5 (Tip)

TYPE OF DISTANCE WITHIN CLADE NESTED CLADE

CLADE BSA2bsa1 (Tip)

TYPE OF DISTANCE WITHIN CLADE NESTED CLADE

CLADE BSA3bsa1 (Tip)

TYPE OF DISTANCE WITHIN CLADE NESTED CLADE

CLADE BSA4bsa1 (Tip)

TYPE OF DISTANCE WITHIN CLADE

CLADE BSA5bsa1 (Tip)

TYPE OF DISTANCE WITHIN CLADE NESTED CLADE

CLADE BSA8bsa1 (Tip)

TYPE OF DISTANCE WITHIN CLADE NESTED CLADE
CLADE BSA1bsa4 (Tip) NESTED CLADE

DISTANCE

33.7826

89.8720

DISTANCE

0.0000

131.2241

DISTANCE

○. $00 \odot \odot$

30.1246

DISTANCE

○. 0000

153.4694

DISTANCE

๑. $000 \odot$

113.3430

DISTANCE

9.7724

26.7616

DISTANCE

○. $00 \odot \odot$

131.2241

DISTANCE

0.0000

23.0271

DISTANCE

๑. . $९ \odot \odot$

115.7834

DISTANCE

○. $0 \odot \odot \odot$

23. 0271

CLADE BSC1bsa1 (Interior)

TYPE OF DISTANCE WITHIN CLADE
PROB . $<=$

0.0048

$\odot .2466$

PROB . > =

0.9952

$\odot .7534$

PROB . $<=$

๑. 0301

$\odot .6781$

PROB . > =

1.0000

$\odot .3310$

PROB . $<=$

○. 8840

$\odot .1946$

PROB . $>=$

1.0000

0.8054

PROB . $<=$

0.8863

$\odot .6866$

PROB . $>=$

1. 0000

$\odot .3134$

PROB . $<=$

0.8820

PROB . > =

$\odot .4254$

1.0000

0.5746

PROB . <=

0.0193

$\odot .0 \odot 65$

PROB . >

0.9807

$\odot .9935$

PROB . $<=$

$\odot .8850$

$\odot .6935$

PROB . $>=$

1. 0000

$\odot .4172$

PROB . $<=$

PROB . > =

0.8806

0.1144

1.0000

1.0000

$$
\begin{array}{rr}
\text { PROB. }<= & \text { PROB. }>= \\
0.8806 & 1.000 \odot \\
0.5061 & 0.4939
\end{array}
$$

PROB . $<=$

๑. 8862

PROB . > =

$\odot .1142$

1.0000

1.0000

PROB . $<=$

PROB . > =

$\odot .1598$ 

NESTED CLADE
265.9588
1.0000
0.0000

PART III. TEST OF INTERIOR VS. TIP CLADES:

$\begin{array}{rrrr}\text { TYPE OF DISTANCE } & \text { I-T } \text { DISTANCE } & \text { PROB. }<= & \text { PROB. }>= \\ \text { WITHIN CLADE } & 37.6573 & 0.1693 & 0.8307 \\ \text { NESTED CLADE } & 87.6102 & 0.9859 & 0.0141\end{array}$

PERMUTATION ANALYSIS OF 1 -4 SOUTHERN ALPS C 1

PART I. EXACT CONTINGENCY TEST:

OBSERVED CHI-SQUARE STATISTIC $=5.0000$

THE PROBABILITY OF A RANDOM CHI-SQUARE BEING GREATER THAN OR EQUAL TO THE OBSERVED CHI-SQUARE $=\quad 0.4035$

PART II. GEOGRAPHIC DISTANCE ANALYSIS:

\begin{tabular}{|c|c|c|c|c|}
\hline \multirow{4}{*}{$\begin{array}{r}\text { CLADE } \\
\text { TYPE }\end{array}$} & \multicolumn{4}{|c|}{ BSC2bsa1 (Interior) } \\
\hline & OF DISTANCE & DISTANCE & PROB . $<=$ & PROB . > = \\
\hline & WITHIN CLADE & 11.6585 & 0.5965 & 0.4035 \\
\hline & NESTED CLADE & 10.5987 & 0.7986 & 0.4035 \\
\hline \multirow{4}{*}{$\begin{array}{l}\text { CLADE } \\
\text { TYPF }\end{array}$} & BSC2bsa8 (Tip & & & \\
\hline & OF DISTANCE & DISTANCE & PROB . $<=$ & PROB . > = \\
\hline & WITHIN CLADE & $\odot .000 \odot$ & $1.0 \odot \odot \odot$ & 1.0000 \\
\hline & NESTED CLADE & 11.1687 & $\odot .4035$ & $\odot .7986$ \\
\hline
\end{tabular}

PART III. TEST OF INTERIOR VS. TIP CLADES:

$\begin{array}{rrrr}\text { TYPE OF DISTANCE } & \text { I-T DISTANCE } & \text { PROB. }<= & \text { PROB. }>= \\ \text { WITHIN CLADE } & 11.6585 & 0.5965 & 0.4035 \\ \text { NESTED CLADE } & -0.5700 & 0.7986 & 0.4035\end{array}$

PERMUTATION ANALYSIS OF 2-1 SOUTHERN ALPS A\&C2 (2) BASED ON 10000 RESAMPLES

PART I. EXACT CONTINGENCY TEST:

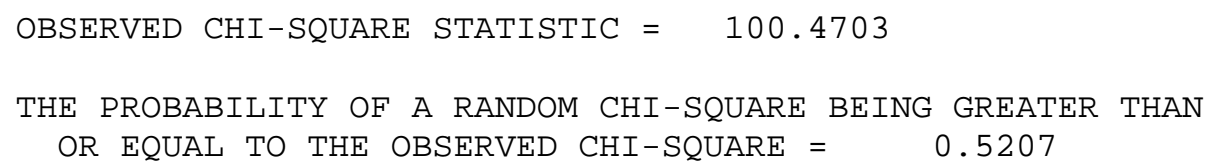

PART II. GEOGRAPHIC DISTANCE ANALYSIS:

\begin{tabular}{|c|c|c|c|c|}
\hline \multicolumn{5}{|c|}{ CLADE 1-1 (Interior) } \\
\hline \multirow{3}{*}{$\begin{array}{l}\text { TYPE } \\
\text { TYUE }\end{array}$} & OF DISTANCE & DISTANCE & PROB . $<=$ & PROB. $>=$ \\
\hline & WITHIN CLADE & 119.5629 & 0.0332 & 0.9668 \\
\hline & NESTED CLADE & 143.3585 & $\odot .0223$ & 0.9777 \\
\hline \multirow{4}{*}{$\begin{array}{r}\text { CLADE } \\
\text { TYPE }\end{array}$} & 1-3 (Tip) & & & \\
\hline & OF DISTANCE & DISTANCE & PROB . $<=$ & PROB . > = \\
\hline & WITHIN CLADE & 0.0000 & 1.0000 & 1.0000 \\
\hline & NESTED CLADE & 192.7435 & 0.7641 & 0.2658 \\
\hline
\end{tabular}

CLADE 1-4 (Tip) 


\begin{tabular}{|c|c|c|c|c|}
\hline \multirow[t]{3}{*}{ TYPE } & OF DISTANCE & DISTANCE & PROB . $<=$ & PROB . $>=$ \\
\hline & WITHIN CLADE & 188.9250 & $\odot .7999$ & 0.2001 \\
\hline & NESTED CLADE & 292.0379 & 1.0000 & 0.0000 \\
\hline \multirow{4}{*}{$\begin{array}{r}\text { CLADE } \\
\text { TYPE }\end{array}$} & 1-5 (Tip) & & & \\
\hline & OF DISTANCE & DISTANCE & PROB . $<=$ & PROB. $>=$ \\
\hline & WITHIN CLADE & 0.0000 & 0.0457 & 1.0000 \\
\hline & NESTED CLADE & 151.7744 & $\odot .4059$ & 0.6088 \\
\hline \multirow{4}{*}{$\begin{array}{r}\text { CLADE } \\
\text { TYPE }\end{array}$} & 1-6 (Tip) & & & \\
\hline & OF DISTANCE & DISTANCE & PROB . $<=$ & PROB. $>=$ \\
\hline & WITHIN CLADE & ๑. . $0 \odot \odot$ & $1 . \odot \odot \odot \odot$ & $1.000 \odot$ \\
\hline & NESTED CLADE & 160.3769 & 0.6040 & 0.4741 \\
\hline \multirow{4}{*}{$\begin{array}{r}\text { CLADE } \\
\text { TYPE }\end{array}$} & 1-7 (Tip) & & & \\
\hline & OF DISTANCE & DISTANCE & PROB . $<=$ & PROB. $>=$ \\
\hline & WITHIN CLADE & 0.0000 & 1.0000 & 1.0000 \\
\hline & NESTED CLADE & 151.7744 & 0.4199 & 0.7086 \\
\hline
\end{tabular}

PART III. TEST OF INTERIOR VS. TIP CLADES:

$\begin{array}{rrrr}\text { TYPE OF DISTANCE } & \text { I-T } \text { DISTANCE } & \text { PROB. }<= & \text { PROB. }>= \\ \text { WITHIN CLADE } & 9.3567 & 0.1029 & 0.8971 \\ \text { NESTED CLADE } & -94.3672 & 0.0027 & 0.9973\end{array}$

PERMUTATION ANALYSIS OF 3-1 SOUTHERN ALPS A\&C (3) BASED ON 10000 RESAMPLES

PART I. EXACT CONTINGENCY TEST:

OBSERVED CHI-SQUARE STATISTIC $=15.4386$

THE PROBABILITY OF A RANDOM CHI-SQUARE BEING GREATER THAN OR EQUAL TO THE OBSERVED CHI-SQUARE $=\quad 0.5903$

PART II. GEOGRAPHIC DISTANCE ANALYSIS:

CLADE 2-1 (Interior)

$\begin{array}{crrr}\text { TYPE OF DISTANCE } & \text { DISTANCE } & \text { PROB. }<= & \text { PROB. }>= \\ \text { WITHIN CLADE } & 168.2964 & 0.5541 & 0.4539 \\ \text { NESTED CLADE } & 165.7079 & 0.2653 & 0.7427\end{array}$

CLADE 2-2 (TIP)

$\begin{array}{rrrr}\text { TYPE OF DISTANCE } & \text { DISTANCE } & \text { PROB. }<= & \text { PROB. }>= \\ \text { WITHIN CLADE } & 2.3863 & 0.0598 & 0.9482 \\ \text { NESTED CLADE } & 186.4657 & 0.7328 & 0.2752\end{array}$

PART III. TEST OF INTERIOR VS. TIP CLADES:

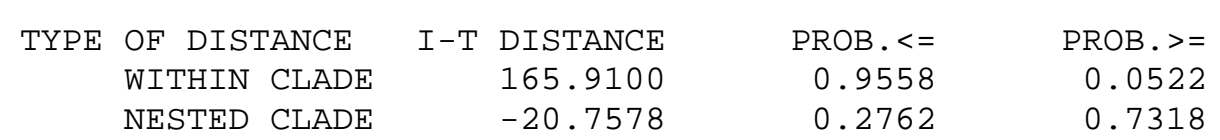

PERMUTATION ANALYSIS OF $1-8$ SOUTHERN ALPS B1 (4)

PART I. EXACT CONTINGENCY TEST: OBSERVED CHI-SQUARE STATISTIC $=40.8000$ 
THE PROBABILITY OF A RANDOM CHI-SQUARE BEING GREATER THAN OR EQUAL TO THE OBSERVED CHI-SQUARE $=\quad 0.3874$

PART II. GEOGRAPHIC DISTANCE ANALYSIS:

\begin{tabular}{|c|c|c|c|c|}
\hline \multirow{4}{*}{$\begin{array}{r}\text { CLADE } \\
\text { TYPE }\end{array}$} & \multicolumn{4}{|c|}{ BSB1bsb1 (Interior) } \\
\hline & OF DISTANCE & DISTANCE & PROB . $<=$ & PROB . $>=$ \\
\hline & WITHIN CLADE & 13.9633 & ๑. 0923 & 0.9077 \\
\hline & NESTED CLADE & 25.0298 & 0.0690 & 0.9310 \\
\hline \multirow{4}{*}{$\begin{array}{r}\text { CLADE } \\
\text { TYPE }\end{array}$} & BSB1bsb2 (Tip) & & & \\
\hline & OF DISTANCE & DISTANCE & PROB . $<=$ & PROB . $>=$ \\
\hline & WITHIN CLADE & 0.0000 & $\odot .8006$ & 1.0000 \\
\hline & NESTED CLADE & 83.8699 & $\odot .9319$ & 0.0681 \\
\hline \multirow{4}{*}{$\begin{array}{r}\text { CLADE } \\
\text { TYPE }\end{array}$} & BSB1bsb3 (Tip) & & & \\
\hline & OF DISTANCE & DISTANCE & PROB . $<=$ & PROB . $>=$ \\
\hline & WITHIN CLADE & 0.0000 & $\odot .8027$ & 1.0000 \\
\hline & NESTED CLADE & 307.2901 & 1.0000 & 0.0675 \\
\hline \multirow{4}{*}{$\begin{array}{r}\text { CLADE } \\
\text { TYPE }\end{array}$} & BSB2bsb1 (Tip) & & & \\
\hline & OF DISTANCE & DISTANCE & PROB . $<=$ & PROB. $>=$ \\
\hline & WITHIN CLADE & 0.0000 & 0.7935 & 1.0000 \\
\hline & NESTED CLADE & 21.6570 & 0.5984 & 0.6081 \\
\hline \multirow{4}{*}{$\begin{array}{r}\text { CLADE } \\
\text { TYPE }\end{array}$} & BSB3bsb1 (Tip) & & & \\
\hline & OF DISTANCE & DISTANCE & PROB . $<=$ & PROB . $>=$ \\
\hline & WITHIN CLADE & $0.000 \odot$ & 0.7893 & 1.0000 \\
\hline & NESTED CLADE & 21.6570 & 0.6025 & 0.6082 \\
\hline \multirow{4}{*}{$\begin{array}{r}\text { CLADE } \\
\text { TYPE }\end{array}$} & BSB4bsb1 (Tip) & & & \\
\hline & OF DISTANCE & DISTANCE & PROB . $<=$ & PROB . $>=$ \\
\hline & WITHIN CLADE & 0.0000 & $\odot .7995$ & 1.0000 \\
\hline & NESTED CLADE & 11.9714 & 0.3338 & 0.6662 \\
\hline
\end{tabular}

PART III. TEST OF INTERIOR VS. TIP CLADES:

$\begin{array}{rrrr}\text { TYPE OF DISTANCE } & \text { I-T } \text { DISTANCE } & \text { PROB. }<= & \text { PROB. }>= \\ \text { WITHIN CLADE } & 13.9633 & 0.0976 & 0.9024 \\ \text { NESTED CLADE } & -64.2593 & 0.0713 & 0.9287\end{array}$

PERMUTATION ANALYSIS OF 3-2 SOUTHERN ALPS B2 (5)

PART I. EXACT CONTINGENCY TEST: OBSERVED CHI-SQUARE STATISTIC $=\quad 7.4667$

THE PROBABILITY OF A RANDOM CHI-SQUARE BEING GREATER THAN OR EQUAL TO THE OBSERVED CHI-SQUARE $=\quad 0.5037$

PART II. GEOGRAPHIC DISTANCE ANALYSIS:

CLADE 2-3 (Tip)

TYPE OF DISTANCE

WITHIN CLADE

NESTED CLADE

$\begin{array}{rrr}\text { DISTANCE } & \text { PROB. }<= & \text { PROB. }>= \\ 40.7353 & 0.1224 & 0.8776 \\ 49.3336 & 0.0000 & 1.0000\end{array}$

CLADE 2-4 (Interior)

TYPE OF DISTANCE WITHIN CLADE NESTED CLADE

$\begin{array}{rrr}\text { DISTANCE } & \text { PROB. }<= & \text { PROB. }>= \\ 0.000 \odot & 0.8099 & 1.0000 \\ 294.9406 & 1.0000 & 0.1224\end{array}$

PART III. TEST OF INTERIOR VS. TIP CLADES: 


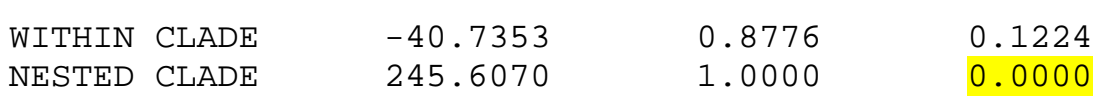

PERMUTATION ANALYSIS OF 4-1 SOUTHERN ALPS ALL (6)

BASED ON 10000 RESAMPLES

PART I. EXACT CONTINGENCY TEST:

OBSERVED CHI-SQUARE STATISTIC $=\quad 45.5469$

THE PROBABILITY OF A RANDOM CHI-SQUARE BEING GREATER THAN OR EQUAL TO THE OBSERVED CHI-SQUARE $=\quad 0.0000$

PART II. GEOGRAPHIC DISTANCE ANALYSIS:

$\begin{array}{rlrrr}\text { CLADE } & 3-1 \text { (Interior) } & & \\ \text { TYPE } & \text { OF DISTANCE } & \text { DISTANCE } & \text { PROB.<= } & \text { PROB. }>= \\ \text { WITHIN CLADE } & 167.7887 & 0.0710 & 0.9290 \\ & \text { NESTED CLADE } & 180.9400 & 0.2285 & 0.7715 \\ & & & \\ \text { CLADE } & \text { 3-2 (Tip) } & & & \\ \text { TYPE OF DISTANCE } & \text { DISTANCE } & \text { PROB.<= } & \text { PROB. >= } \\ \text { WITHIN CLADE } & 59.2913 & 0.00 \odot \odot & 1.000 \odot \\ \text { NESTED CLADE } & 210.1687 & 0.9369 & 0.0631\end{array}$

PART III. TEST OF INTERIOR VS. TIP CLADES:

\begin{tabular}{|c|c|c|c|c|c|}
\hline TYPE & OF DISTANCE & $\mathrm{I}-\mathrm{T}$ & DISTANCE & PROB . $<=$ & PROB. $>=$ \\
\hline & WITHIN CLADE & & 108.4975 & 0.9957 & $\odot .0 \odot 43$ \\
\hline & NESTED CLADF & & -29.2287 & 0.0999 & $\odot .900$ \\
\hline
\end{tabular}

PERMUTATION ANALYSIS OF 1-10 WAITAKI 1 (7)

PART I. EXACT CONTINGENCY TEST:

OBSERVED CHI-SQUARE STATISTIC $=25.2353$

THE PROBABILITY OF A RANDOM CHI-SQUARE BEING GREATER THAN OR EQUAL TO THE OBSERVED CHI-SQUARE $=\quad 0.9075$

PART II. GEOGRAPHIC DISTANCE ANALYSIS:

CLADE BW1bW1 (Interior)

WITHIN CLADE 26.1783

NESTED CLADE 26.4452

PROB. $<=\quad$ PROB. $>=$

$\odot .0699 \quad 0.9301$

$0.0491 \quad 0.9509$

CLADE BW2bW1 (Tip)

TYPE OF DISTANCE WITHIN CLADE

DISTANCE

PROB . $<=$

PROB . > = NESTED CLADE

0.0000

0.8149

1. 0000

34.5905

0.7601

0.5798

CLADE BW4bW1 (Tip)

TYPE OF DISTANCE

WITHIN CLADE

DISTANCE

0.0000

PROB . $<=$

0.8103

PROB . $>=$ NESTED CLADE

34.5905

$\odot .7574$

1. 0000

$\odot .5583$

CLADE BW7bW1 (Tip)

TYPE OF DISTANCE

PROB . $<=$

PROB . > = 


$\begin{array}{llll}\text { WITHIN CLADE } & 10.5012 & 0.6993 & 0.3038 \\ \text { NESTED CLADE } & 70.0491 & 0.9665 & 0.0335\end{array}$

PART III. TEST OF INTERIOR VS. TIP CLADES:

$\begin{array}{rrrr}\text { TYPE OF DISTANCE } & \text { I-T DISTANCE } & \text { PROB. }<= & \text { PROB. }>= \\ \text { WITHIN CLADE } & 20.9277 & 0.2389 & 0.7611 \\ \text { NESTED CLADE } & -25.8746 & 0.0239 & 0.9761\end{array}$

PERMUTATION ANALYSIS OF 1-12 WAITAKI 2 (8)

PART I. EXACT CONTINGENCY TEST:

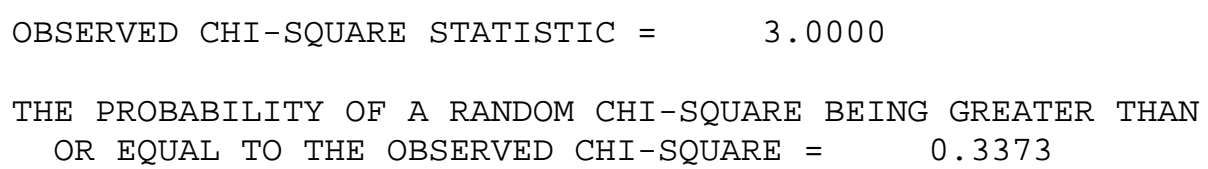

PART II. GEOGRAPHIC DISTANCE ANALYSIS:

$\begin{array}{rlrr}\text { CLADE BW3bw2 (Interior) } & & & \\ \text { TYPE OF DISTANCE } & \text { DISTANCE } & \text { PROB. }<= & \text { PROB. }>= \\ \text { WITHIN CLADE } & 0.0000 & 0.3373 & 1.0000 \\ \text { NESTED CLADE } & 21.8437 & 0.3373 & 1.0000 \\ & & & \\ \text { CLADE BW10bW2 (Tip) } & & & \\ \text { TYPE } \text { OF DISTANCE } & \text { DISTANCE } & \text { PROB.<= } & \text { PROB. }>= \\ \text { WITHIN CLADE } & 0.0000 & 1.0000 & 1.0000 \\ \text { NESTED CLADE } & 43.6253 & 0.6627 & 0.3373\end{array}$

PART III. TEST OF INTERIOR VS. TIP CLADES:

$\begin{array}{rrrr}\text { TYPE OF DISTANCE } & \text { I-T } \text { DISTANCE } & \text { PROB. }<= & \text { PROB. }>= \\ \text { WITHIN CLADE } & 0.00 \odot \odot & 0.3373 & 1.000 \odot \\ \text { NESTED CLADE } & -21.7816 & 0.3373 & 0.6627\end{array}$

PERMUTATION ANALYSIS OF 2-5 WAITAKI 3 (BOTH INTERIOR?) (9)

PART I. EXACT CONTINGENCY TEST:

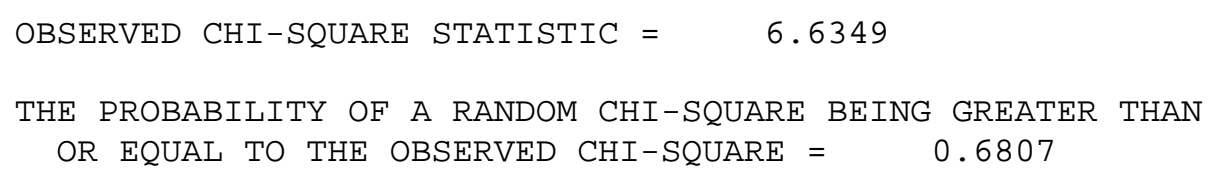

PART II. GEOGRAPHIC DISTANCE ANALYSIS:

$\begin{array}{rlrr}\text { CLADE } & 1-10 \text { (Interior) } & & \\ \text { TYPE OF DISTANCE } & \text { DISTANCE } & \text { PROB. }<= & \text { PROB. }>= \\ \text { WITHIN CLADE } & 29.5144 & 0.6375 & 0.3625 \\ \text { NESTED CLADE } & 29.5134 & 0.5937 & 0.4063 \\ & & & \\ \text { CLADE 1-11 (Interior) } & & & \\ \text { TYPE OF DISTANCE } & \text { DISTANCE } & \text { PROB. }<= & \text { PROB. >= } \\ \text { WITHIN CLADE } & 0.00 \odot \odot & 0.7720 & 1.000 \% \\ \text { NESTED CLADE } & 22.7487 & 0.2728 & 0.8639\end{array}$


PERMUTATION ANALYSIS OF 2-7 WAITAKI 4(10)

PART I. EXACT CONTINGENCY TEST:

OBSERVED CHI-SQUARE STATISTIC $=4.0 \odot \odot \odot$

THE PROBABILITY OF A RANDOM CHI-SQUARE BEING GREATER THAN OR EQUAL TO THE OBSERVED CHI-SQUARE $=\quad 0.2460$

PART II. GEOGRAPHIC DISTANCE ANALYSIS:

CLADE 1-13 (Interior)

TYPE OF DISTANCE DISTANCE WITHIN CLADE $\quad 0.0000$ NESTED CLADE 18.8662

PROB . $<=$ PROB . $>=$

DISTANCE

๑. $00 \odot \odot$

0.2460

1.0000

CLADE 1-14 (Interior)

TYPE OF DISTANCE WITHIN CLADE NESTED CLADE

8.0844

PROB . $<=$

$1.000 \odot$

$\odot .2460$

$\odot .2460$

NO INTERIOR/TIP CLADES EXIST IN THIS GROUP

PERMUTATION ANALYSIS OF 3-3 WAITAKI 5 (11)

PART I. EXACT CONTINGENCY TEST:

OBSERVED CHI-SQUARE STATISTIC $=20.2652$

THE PROBABILITY OF A RANDOM CHI-SQUARE BEING GREATER THAN OR EQUAL TO THE OBSERVED CHI-SQUARE $=0.1072$

PART II. GEOGRAPHIC DISTANCE ANALYSIS:

CLADE 2-5 (Interior)

TYPE OF DISTANCE WITHIN CLADE

DISTANCE

29.4537

PROB . $<=$

$\odot .0551$

PROB . > = NESTED CLADE

29.8331

0.0583

PROB . > =

$1.000 \odot$

1. 0000

CLADE 2-6 (Tip)

TYPE OF DISTANCE WITHIN CLADE

DISTANCE

PROB . $<=$

$\odot .9449$

NESTED CLADE

29.1043

0.7945

$\odot .8340$

$\odot .9417$

PART III. TEST OF INTERIOR VS. TIP CLADES:

$\begin{array}{rrrr}\text { TYPE OF DISTANCE } & \text { I-T } \text { DISTANCE } & \text { PROB. }<= & \text { PROB. }>= \\ \text { WITHIN CLADE } & 0.3494 & 0.1764 & 0.8236 \\ \text { NESTED CLADE } & -13.9453 & 0.1660 & 0.8340\end{array}$

PERMUTATION ANALYSIS OF 3-4 WAITAKI 6 (12)

BASED ON 10000 RESAMPLES

PART I. EXACT CONTINGENCY TEST:
OBSERVED CHI-SQUARE STATISTIC =
0.6000
THE PROBABILITY OF A RANDOM CHI-SQUARE BEING GREATER THAN OR EQUAL TO THE OBSERVED CHI-SQUARE $=\quad 1.0000$ 
PART II. GEOGRAPHIC DISTANCE ANALYSIS:

CLADE 2-7 (Interior)

TYPE OF DISTANCE

WITHIN CLADE

DISTANCE

PROB . $<=$

PROB . > = NESTED CLADE

14.8257

1. $.0 \odot \odot$

$\odot .000 \%$

16.4730

$\odot .6705$

1. $.00 \odot$

CLADE 2-8 (Tip)

TYPE OF DISTANCE WITHIN CLADE

DISTANCE

0.0000

PROB . $<=$

$\odot .6705$

PROB . > =

20.5927

1. 0000

1.0000

0.0000

PART III. TEST OF INTERIOR VS. TIP CLADES:

$\begin{array}{rrrr}\text { TYPE OF DISTANCE } & \text { I -T DISTANCE } & \text { PROB. }<= & \text { PROB. }>= \\ \text { WITHIN CLADE } & 14.8257 & 1.000 \odot & 0.000 \odot \\ \text { NESTED CLADE } & -4.1196 & 0.000 \odot & 1.0000\end{array}$

PERMUTATION ANALYSIS OF 4-2 WAITAKI ALL (13)

PART I. EXACT CONTINGENCY TEST:

OBSERVED CHI-SQUARE STATISTIC $=56.6181$

THE PROBABILITY OF A RANDOM CHI-SQUARE BEING GREATER THAN OR EQUAL TO THE OBSERVED CHI-SQUARE $=\quad 0.0102$

PART II. GEOGRAPHIC DISTANCE ANALYSIS:

CLADE 3-3 (Interior)

TYPE OF DISTANCE

WITHIN CLADE

DISTANCE

30.3831

PROB . $<=$

$\odot .0009$

NESTED CLADE

33.2401

$\odot .0007$

PROB . > =

$\odot .9991$

$\odot .9993$

CLADE 3-4 (Tip)

TYPE OF DISTANCE WITHIN CLADE

DISTANCE

13. 1009

PROB . $<=$

๑. 0110

PROB . >

92.7303

$\odot .9666$

0.9890 NESTED CLADE

DISTANCE

PROB . $<=$

0.8364

1. $0 \odot \odot \odot$

0.0334

CLADE 3-5 (Tip)

TYPE OF DISTANCE

WITHIN CLADE

NESTED CLADE

143.0661

PROB . > =

1.0000

0.0000

PART III. TEST OF INTERIOR VS. TIP CLADES:

$\begin{array}{rrrr}\text { TYPE OF DISTANCE } & \text { I-T DISTANCE } & \text { PROB. }<= & \text { PROB. }>= \\ \text { WITHIN CLADE } & 19.1538 & 0.4653 & 0.5347 \\ \text { NESTED CLADE } & -66.6810 & 0.0037 & 0.9963\end{array}$

PERMUTATION ANALYSIS OF 1-18 MARLBOROUGH 1 (14)

PART I. EXACT CONTINGENCY TEST:
OBSERVED CHI-SQUARE STATISTIC =
$2 . \odot \odot \odot \odot$
THE PROBABILITY OF A RANDOM CHI-SQUARE BEING GREATER THAN OR EQUAL TO THE OBSERVED CHI-SQUARE =
$1 . \odot \odot \odot \odot$

PART II. GEOGRAPHIC DISTANCE ANALYSIS: 


\begin{tabular}{|c|c|c|c|c|}
\hline \multirow{4}{*}{$\begin{array}{r}\text { CLADE } \\
\text { TYPE }\end{array}$} & \multicolumn{4}{|c|}{ BM1bm1 (Interior) } \\
\hline & OF DISTANCE & DISTANCE & PROB. $<=$ & PROB . $>=$ \\
\hline & WITHIN CLADE & 0.0000 & 0.4889 & 1.0000 \\
\hline & NESTED CLADE & 9.3974 & 0.0000 & 1.0000 \\
\hline \multirow{4}{*}{$\begin{array}{r}\text { CLADE } \\
\text { TYPE }\end{array}$} & BM1bm4 (Tip) & & & \\
\hline & OF DISTANCE & DISTANCE & PROB . $<=$ & PROB . $>=$ \\
\hline & WITHIN CLADE & 0.0000 & 0.5008 & 1.0000 \\
\hline & NESTED CLADE & 9.4021 & 1.0000 & 0.5008 \\
\hline \multirow{4}{*}{$\begin{array}{r}\text { CLADE } \\
\text { TYPE }\end{array}$} & BM3bm1 (Tip) & & & \\
\hline & OF DISTANCE & DISTANCE & PROB . $<=$ & PROB. $>=$ \\
\hline & WITHIN CLADE & 9.3997 & 0.3349 & 0.6651 \\
\hline & NESTED CLADE & 9.3997 & 0.1623 & 0.8377 \\
\hline
\end{tabular}

PART III. TEST OF INTERIOR VS. TIP CLADES:

$\begin{array}{rrrr}\text { TYPE OF DISTANCE } & \text { I-T } \text { DISTANCE } & \text { PROB. }<= & \text { PROB. }>= \\ \text { WITHIN CLADE } & -6.2665 & 0.3266 & 0.6734 \\ \text { NESTED CLADE } & -0.0032 & 0.000 \odot & 1.0000\end{array}$

PERMUTATION ANALYSIS OF 1-20 MARLBOROUGH 3 (15)

PART I. EXACT CONTINGENCY TEST: OBSERVED CHI-SQUARE STATISTIC $=\quad 3.0000$
THE PROBABILITY OF A RANDOM CHI-SQUARE BEING GREATER THAN
OR EQUAL TO THE OBSERVED CHI-SQUARE $=0.6687$

PART II. GEOGRAPHIC DISTANCE ANALYSIS:

\begin{tabular}{|c|c|c|c|c|c|}
\hline \multirow{4}{*}{$\begin{array}{r}\text { CLADE } \\
\text { TYPE }\end{array}$} & \multicolumn{5}{|c|}{ BM2bm1 (Interior) } \\
\hline & OF DIS & ГANCE & DISTANCE & PROB . $<=$ & PROB . $>=$ \\
\hline & WITHIN & CLADE & 18.2688 & 0.6622 & 0.6687 \\
\hline & NESTED & CLADE & 16.2397 & 0.6622 & 0.6687 \\
\hline \multicolumn{6}{|l|}{ CLADE } \\
\hline \multirow[t]{3}{*}{ TYPE } & \multicolumn{2}{|c|}{ OF DISTANCE } & DISTANCE & PROB . $<=$ & PROB..$>=$ \\
\hline & WITHIN & CLADE & 0.0000 & ๑. 6691 & 1.0000 \\
\hline & NESTED & CLADE & 27.4448 & 0.3378 & $\odot .6622$ \\
\hline
\end{tabular}

PART III. TEST OF INTERIOR VS. TIP CLADES:

$\begin{array}{rrrr}\text { TYPE OF DISTANCE } & \text { I-T DISTANCE } & \text { PROB. }<= & \text { PROB. }>= \\ \text { WITHIN CLADE } & 18.2688 & 0.6622 & 0.3378 \\ \text { NESTED CLADE } & -11.2051 & 0.6622 & 0.3378\end{array}$

PERMUTATION ANALYSIS OF 1-22 MARLBOROUGH 2 (16)

PART I. EXACT CONTINGENCY TEST:

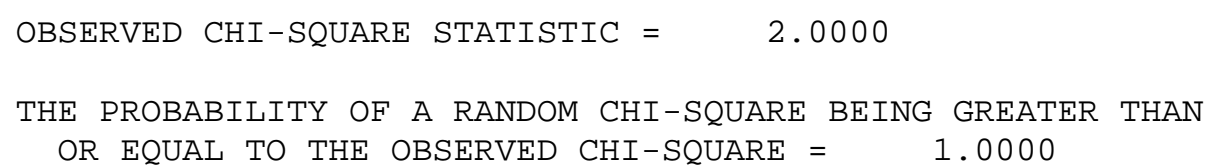

PART II. GEOGRAPHIC DISTANCE ANALYSIS:

CLADE BM1bm2 (Interior) 


$\begin{array}{rrrr}\text { TYPE OF DISTANCE } & \text { DISTANCE } & \text { PROB. }<= & \text { PROB. }>= \\ \text { WITHIN CLADE } & 0.00 \odot \odot & 1.00 \odot \odot & 1.00 \odot \odot \\ \text { NESTED CLADE } & 2.9641 & 1.00 \odot \odot & 0.4944 \\ & & & \\ \text { CLADE BM5bm2 (Tip) } & & & \\ \text { TYPE OF DISTANCE } & \text { DISTANCE } & \text { PROB. }<= & \text { PROB. }>= \\ \text { WITHIN CLADE } & 0.00 \odot \odot & 1.0 \odot \odot \odot & 1.00 \odot \odot \\ \text { NESTED CLADE } & 2.9637 & 0.00 \odot \odot & 1.00 \odot \odot\end{array}$

PART III. TEST OF INTERIOR VS. TIP CLADES:

$\begin{array}{rrrr}\text { TYPE OF DISTANCE } & \text { I-T DISTANCE } & \text { PROB. }<= & \text { PROB. }>= \\ \text { WITHIN CLADE } & 0.00 \odot \odot & 1.00 \odot \odot & 1.00 \odot \odot \\ \text { NESTED CLADE } & 0.0003 & 1.00 \odot \odot & 0.000 \odot\end{array}$

PERMUTATION ANALYSIS OF 2-10 MARLBOROUGH 4 (17)

PART I. EXACT CONTINGENCY TEST:

OBSERVED CHI-SQUARE STATISTIC $=5.000 \%$

THE PROBABILITY OF A RANDOM CHI-SQUARE BEING GREATER THAN OR EQUAL TO THE OBSERVED CHI-SQUARE $=\quad 0.1922$

PART II. GEOGRAPHIC DISTANCE ANALYSIS:

\begin{tabular}{|c|c|c|c|c|}
\hline \multirow{2}{*}{$\begin{array}{r}\text { CLADE } \\
\text { TYPE }\end{array}$} & \multicolumn{4}{|c|}{ 1-18 (Interior) } \\
\hline & OF DISTANCE & DISTANCE & PROB . $<=$ & PROB . $>=$ \\
\hline & WITHIN CLADE & 9.3997 & $\odot .0000$ & 1.0000 \\
\hline & NESTED CLADE & 15.2055 & 0.1922 & 0.8078 \\
\hline \multicolumn{5}{|c|}{ CLADE 1-19 (Tip) } \\
\hline \multirow[t]{3}{*}{ TYPE } & OF DISTANCE & DISTANCE & PROB . $<=$ & PROB . $>=$ \\
\hline & WITHIN CLADE & $\odot .0 \odot \odot \odot$ & 0.6010 & $1.00 \odot \odot$ \\
\hline & NESTED CLADE & 20.2620 & 0.4088 & $\odot .5912$ \\
\hline
\end{tabular}

PART III. TEST OF INTERIOR VS. TIP CLADES:

$\begin{array}{rrrr}\text { TYPE OF DISTANCE } & \text { I-T DISTANCE } & \text { PROB. }<= & \text { PROB. }>= \\ \text { WITHIN CLADE } & 9.3997 & 0.00 \odot \odot & 1.000 \odot \\ \text { NESTED CLADE } & -5.0566 & 0.5912 & 0.4088\end{array}$

PERMUTATION ANALYSIS OF 2-11 MARLBOROUGH 5 (18)

PART I. EXACT CONTINGENCY TEST:

OBSERVED CHI-SQUARE STATISTIC $=1.3333$

THE PROBABILITY OF A RANDOM CHI-SQUARE BEING GREATER THAN OR EQUAL TO THE OBSERVED CHI-SQUARE $=1.0000$

PART II. GEOGRAPHIC DISTANCE ANALYSIS:

$\begin{array}{rlrrr}\text { CLADE } & 1-20 \text { (Interior) } & & \\ \text { TYPE OF DISTANCE } & \text { DISTANCE } & \text { PROB.<= } & \text { PROB. >= } \\ \text { WITHIN CLADE } & 18.2770 & 0.0000 & 1.0000 \\ \text { NESTED CLADE } & 21.5365 & 0.7506 & 0.2494 \\ & & & \\ \text { CLADE } 1-21 \text { (Tip) } & & & \\ \text { TYPE OF DISTANCE } & \text { DISTANCE } & \text { PROB.<= } & \text { PROB. >= } \\ \text { WITHIN CLADE } & 0.0000 & 0.7495 & 1.0000\end{array}$



NESTED CLADE
26.3391
0.7495
0.2505

PART III. TEST OF INTERIOR VS. TIP CLADES:

$\begin{array}{rrrr}\text { TYPE OF DISTANCE } & \text { I-T DISTANCE } & \text { PROB. }<= & \text { PROB. }>= \\ \text { WITHIN CLADE } & 18.2770 & 0.0 \odot \odot \odot & 1.00 \odot \odot \\ \text { NESTED CLADE } & -4.8027 & 0.2505 & 0.7495\end{array}$

PERMUTATION ANALYSIS OF 2-12 MARLBOROUGH 6 (19)

PART I. EXACT CONTINGENCY TEST:

OBSERVED CHI-SQUARE STATISTIC $=\quad 3.000 \%$

THE PROBABILITY OF A RANDOM CHI-SQUARE BEING GREATER THAN OR EQUAL TO THE OBSERVED CHI-SQUARE $=1.0000$

PART II. GEOGRAPHIC DISTANCE ANALYSIS:

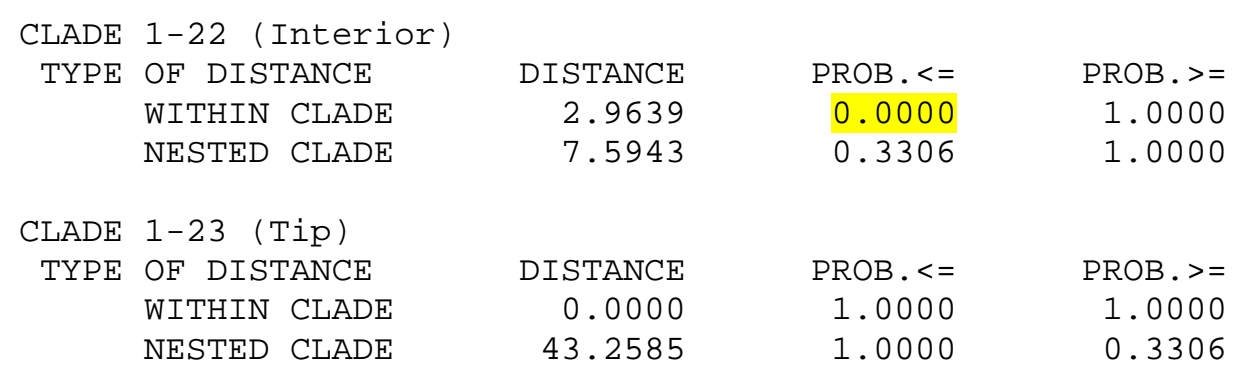

PART III. TEST OF INTERIOR VS. TIP CLADES:

$\begin{array}{rrrr}\text { TYPE OF DISTANCE } & \text { I-T } \text { DISTANCE } & \text { PROB. }<= & \text { PROB. }>= \\ \text { WITHIN CLADE } & 2.9639 & 0.000 \odot & 1.000 \odot \\ \text { NESTED CLADE } & -35.6641 & 0.3306 & 1.000 \odot\end{array}$

PERMUTATION ANALYSIS OF 3-6 MARLBOROUGH 7 (20)

PART I. EXACT CONTINGENCY TEST:
OBSERVED CHI-SQUARE STATISTIC =
6.3000
THE PROBABILITY OF A RANDOM CHI-SQUARE BEING GREATER THAN OR EQUAL TO THE OBSERVED CHI-SQUARE $=\quad 0.2865$

PART II. GEOGRAPHIC DISTANCE ANALYSIS:

\begin{tabular}{|c|c|c|c|c|}
\hline \multirow{4}{*}{$\begin{array}{r}\text { CLADE } \\
\text { TYPE }\end{array}$} & \multicolumn{4}{|c|}{ 2-10 (Interior) } \\
\hline & OF DISTANCE & DISTANCE & PROB . $<=$ & PROB . $>=$ \\
\hline & WITHIN CLADE & 17.3726 & 0.4904 & 0.5096 \\
\hline & NESTED CLADE & 18.1013 & ○. 2918 & 0.7082 \\
\hline \multicolumn{5}{|c|}{ CLADE 2-11 (Tip) } \\
\hline \multirow[t]{3}{*}{ TYPE } & OF DISTANCE & DISTANCE & PROB . $<=$ & PROB. $>=$ \\
\hline & WITHIN CLADE & 22.5656 & 0.8247 & 0.2014 \\
\hline & NESTED CLADE & 21.8459 & 0.6463 & $\odot .3537$ \\
\hline
\end{tabular}

PART III. TEST OF INTERIOR VS. TIP CLADES:

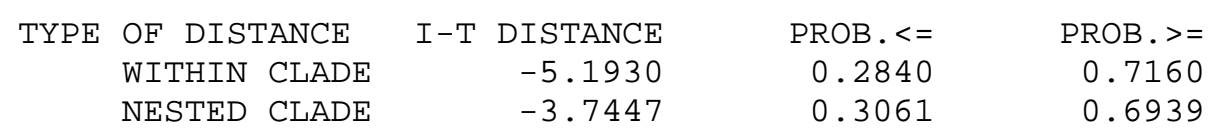


PART I. EXACT CONTINGENCY TEST:

OBSERVED CHI-SQUARE STATISTIC $=\quad 8.0000$

THE PROBABILITY OF A RANDOM CHI-SQUARE BEING GREATER THAN OR EQUAL TO THE OBSERVED CHI-SQUARE $=\quad 0.0143$

PART II. GEOGRAPHIC DISTANCE ANALYSIS:

CLADE 2-12 (Interior)

TYPE OF DISTANCE WITHIN CLADE

DISTANCE

12.6892

37.0896

PROB . $<=$

๑. 1971

0.0143

PROB . $>=$

0.8172

NESTED CLADE

DISTANCE

๑. $0 \odot \odot \odot$

PROB . $<=$

0.0143

1. 0000

$\odot .9857$

CLADE 2-13 (Tip)

TYPE OF DISTANCE

WITHIN CLADE

77.1033

PROB . > =

1.0000

0.0143

PART III. TEST OF INTERIOR VS. TIP CLADES:

$\begin{array}{rrrr}\text { TYPE OF DISTANCE } & \text { I-T DISTANCE } & \text { PROB. }<= & \text { PROB. }>= \\ \text { WITHIN CLADE } & 12.6892 & 1.0000 & 0.0143 \\ \text { NESTED CLADE } & -40.0137 & 0.0143 & 0.9857\end{array}$

PERMUTATION ANALYSIS OF 3-8 KAIKOURA (22)

PART I. EXACT CONTINGENCY TEST:

OBSERVED CHI-SQUARE STATISTIC $=2.0000$

THE PROBABILITY OF A RANDOM CHI-SQUARE BEING GREATER THAN OR EQUAL TO THE OBSERVED CHI-SQUARE $=\quad 1.000 \%$

PART II. GEOGRAPHIC DISTANCE ANALYSIS:

CLADE 2-14 (Tip)

TYPE OF DISTANCE WITHIN CLADE NESTED CLADE
DISTANCE

○. . $000 \%$

14.0352

DISTANCE

○. $000 \odot$

14.0341
PROB . $<=$

1. 0000

$\odot .4902$

PROB. $>=$

1.0000

0.5098

CLADE 2-15 (Interior)

TYPE OF DISTANCE WITHIN CLADE NESTED CLADE
PROB . $<=$

1.0000

$\odot .5098$

PROB . $>=$

1.0000

1.0000

PART III. TEST OF INTERIOR VS. TIP CLADES:
TYPE OF DISTANCE WITHIN CLADE
I - T DISTANCE
0. 0000
PROB . $<=$
PROB . > = NESTED CLADE
$-\odot .0011$
$1.0 \odot \odot \odot$
1. 0000
0.4902

PERMUTATION ANALYSIS OF 4-4 MARLBOROUGH 7 + NELSON + KAIKOURA (23)

PART I. EXACT CONTINGENCY TEST: 
OBSERVED CHI-SQUARE STATISTIC $=10.0000$

THE PROBABILITY OF A RANDOM CHI-SQUARE BEING GREATER THAN OR EQUAL TO THE OBSERVED CHI-SQUARE $=\quad 0.2987$

PART II. GEOGRAPHIC DISTANCE ANALYSIS:

$\begin{array}{rlrr}\text { CLADE } & \text { 3-7 (Interior) } & & \\ \text { TYPE OF DISTANCE } & \text { DISTANCE } & \text { PROB. }<= & \text { PROB. }>= \\ \text { WITHIN CLADE } & 52.1232 & 0.7011 & 0.2989 \\ \text { NESTED CLADE } & 49.3869 & 0.6989 & 0.3011 \\ & & & \\ \text { CLADE } 3-8 \text { (Tip) } & & & \\ \text { TYPE OF DISTANCE } & \text { DISTANCE } & \text { PROB.<= } & \text { PROB.>= } \\ \text { WITHIN CLADE } & 0.0000 & 1.0000 & 1.0000 \\ \text { NESTED CLADE } & 51.3219 & 0.3011 & 0.6989\end{array}$

PART III. TEST OF INTERIOR VS. TIP CLADES:

$\begin{array}{rrrr}\text { TYPE OF DISTANCE } & \text { I-T DISTANCE } & \text { PROB. }<= & \text { PROB. }>= \\ \text { WITHIN CLADE } & 52.1232 & 0.7011 & 0.2989 \\ \text { NESTED CLADE } & -1.9349 & 0.6989 & 0.3011\end{array}$

PERMUTATION ANALYSIS OF 5-1 ALL SOUTH ISLAND (24)

BASED ON 10000 RESAMPLES

PART I. EXACT CONTINGENCY TEST:

\begin{abstract}
OBSERVED CHI-SQUARE STATISTIC $=303.0815$
THE PROBABILITY OF A RANDOM CHI-SQUARE BEING GREATER THAN OR EQUAL TO THE OBSERVED CHI-SQUARE $=\quad 0.00 \odot \odot$
\end{abstract}

PART II. GEOGRAPHIC DISTANCE ANALYSIS:

\begin{tabular}{|c|c|c|c|c|}
\hline CLADE & 4-1 (Tip) & & & \\
\hline TYPE & OF DISTANCE & DISTANCE & PROB . $<=$ & PROB . $>=$ \\
\hline & WITHIN CLADE & 185.9890 & 0.9334 & 0.0666 \\
\hline & NESTED CLADE & 179.2293 & 0.7489 & 0.2511 \\
\hline CLADE & 4-2 (Tip) & & & \\
\hline TYPE & OF DISTANCE & DISTANCE & PROB . $<=$ & PROB . $>=$ \\
\hline & WITHIN CLADE & 49.3332 & 0.0000 & 1.0000 \\
\hline & NESTED CLADE & 116.0554 & 0.0000 & 1.0000 \\
\hline CLADE & 4-3 (Interior) & & & \\
\hline TYPE & OF DISTANCE & DISTANCE & PROB . $<=$ & PROB . $>=$ \\
\hline & WITHIN CLADE & 19.9736 & $\odot .0000$ & 1.0000 \\
\hline & NESTED CLADE & 252.1244 & 0.9865 & 0.0135 \\
\hline CLADE & 4-4 (Tip) & & & \\
\hline TYPE & OF DISTANCE & DISTANCE & PROB . $<=$ & PROB. $>=$ \\
\hline & WITHIN CLADE & 49.7497 & $\odot .0002$ & 0.9998 \\
\hline & NESTED CLADE & 216.5033 & 0.9087 & 0.0913 \\
\hline
\end{tabular}

PART III. TEST OF INTERIOR VS. TIP CLADES:

$\begin{array}{rrrr}\text { TYPE OF DISTANCE } & \text { I-T DISTANCE } & \text { PROB. }<= & \text { PROB. }>= \\ \text { WITHIN CLADE } & -108.2966 & 0.0074 & 0.9926\end{array}$


ERMUTATION ANALYSIS OF 1-28 NORTH ISLAND 1 BASED ON $1000 \%$ RESAMPLES

PART I. EXACT CONTINGENCY TEST:

OBSERVED CHI-SQUARE STATISTIC = 6.6667

THE PROBABILITY OF A RANDOM CHI-SQUARE BEING GREATER THAN OR EQUAL TO THE OBSERVED CHI-SQUARE $=0.7033$

PART II. GEOGRAPHIC DISTANCE ANALYSIS:

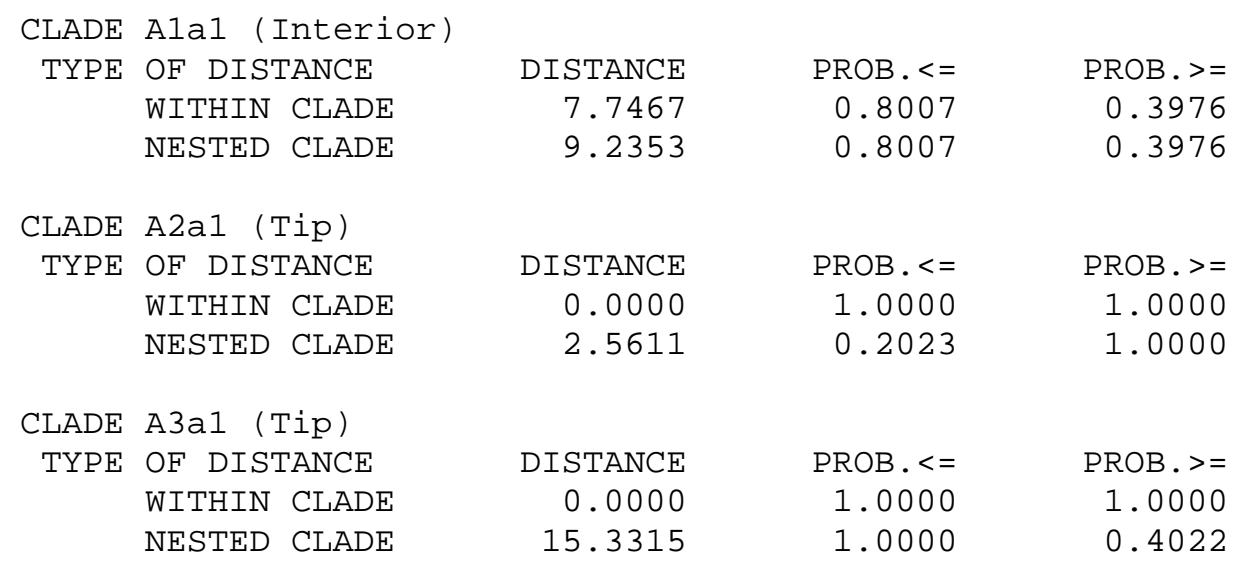

PART III. TEST OF INTERIOR VS. TIP CLADES:

$\begin{array}{rrrr}\text { TYPE OF DISTANCE } & \text { I -T DISTANCE } & \text { PROB . }<= & \text { PROB. }>= \\ \text { WITHIN CLADE } & 7.7467 & 0.8007 & 0.3976 \\ \text { NESTED CLADE } & 0.2889 & 0.8007 & 0.3976\end{array}$

PERMUTATION ANALYSIS OF 2-16 NORTH ISLAND ALL BASED ON 10000 RESAMPLES

PART I. EXACT CONTINGENCY TEST:
OBSERVED CHI-SQUARE STATISTIC $=1.2000$
THE PROBABILITY OF A RANDOM CHI-SQUARE BEING GREATER THAN OR EQUAL TO THE OBSERVED CHI-SQUARE $=1.0000$

PART II. GEOGRAPHIC DISTANCE ANALYSIS: $\begin{aligned} & \text { CLADE } 1-28 \text { (Tip) } \\ & \text { TYPE } \text { OF DISTANCE } \\ & \text { WITHIN CLADE } \\ & \text { NESTED CLADE }\end{aligned}$
PROB . $<=$

$\odot .4985$

$\odot .4985$
PROB . >=

1.0000

1.0000 


$\begin{array}{rlrrr}\text { CLADE } & 1-29 \text { (Tip) } & & & \\ \text { TYPE } & \text { OF DISTANCE } & \text { DISTANCE } & \text { PROB. }<= & \text { PROB. }>= \\ \text { WITHIN CLADE } & 0.0000 & 1.0000 & 1.0000 \\ \text { NESTED CLADE } & 14.0710 & 1.0000 & 0.4985\end{array}$

NO INTERIOR/TIP CLADES EXIST IN THIS GROUP

PERMUTATION ANALYSIS OF 1 -30 OTAGO NORTH 1

BASED ON $1000 \odot$ RESAMPLES

PART I. EXACT CONTINGENCY TEST:

OBSERVED CHI-SQUARE STATISTIC = 8.0294

THE PROBABILITY OF A RANDOM CHI-SQUARE BEING GREATER THAN OR EQUAL TO THE OBSERVED CHI-SQUARE $=\quad 0.5791$

PART II. GEOGRAPHIC DISTANCE ANALYSIS:

$\begin{array}{rrrr}\text { CLADE CN1Cn1 (Interior) } & & & \\ \text { TYPE OF DISTANCE } & \text { DISTANCE } & \text { PROB.<= } & \text { PROB. }>= \\ \text { WITHIN CLADE } & 28.2224 & 0.2028 & 0.8102 \\ \text { NESTED CLADE } & 27.4555 & 0.2587 & 0.7543 \\ & & & \\ \text { CLADE CN1Cn3 (Tip) } & & & \\ \text { TYPE OF DISTANCE } & \text { DISTANCE } & \text { PROB.<= } & \text { PROB. >= } \\ \text { WITHIN CLADE } & 0.000 \odot & 0.2336 & 1.000 \% \\ \text { NESTED CLADE } & 41.6413 & 0.9077 & 0.2297 \\ & & & \\ \text { CLADE CN2Cn1 (Tip) } & & & \\ \text { TYPE OF DISTANCE } & \text { DISTANCE } & \text { PROB.<= } & \text { PROB.>= } \\ \text { WITHIN CLADE } & 0.0 \odot \odot \odot & 0.2406 & 1.00 \odot \odot \\ \text { NESTED CLADE } & 41.6413 & 0.9025 & 0.2328\end{array}$

PART III. TEST OF INTERIOR VS. TIP CLADES:

$\begin{array}{rrrr}\text { TYPE OF DISTANCE } & \text { I -T DISTANCE } & \text { PROB. }<= & \text { PROB. }>= \\ \text { WITHIN CLADE } & 28.2224 & 0.8457 & 0.1673 \\ \text { NESTED CLADE } & -14.1858 & 0.1956 & 0.8174\end{array}$

PERMUTATION ANALYSIS OF 1-31 OTAGO NORTH 2 BASED ON 10000 RESAMPLES

PART I. EXACT CONTINGENCY TEST:

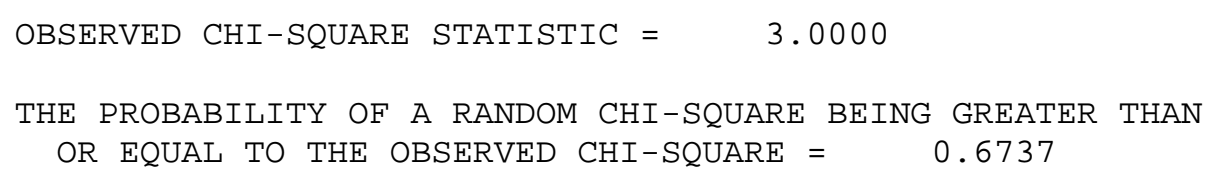


PART II. GEOGRAPHIC DISTANCE ANALYSIS:

\begin{tabular}{|c|c|c|c|c|}
\hline \multirow{4}{*}{$\begin{array}{r}\text { CLADE } \\
\text { TYPE }\end{array}$} & \multicolumn{4}{|c|}{ CN1cn2 (Interior) } \\
\hline & OF DISTANCE & DISTANCE & PROB . $<=$ & PROB. $>=$ \\
\hline & WITHIN CLADE & 11.6594 & $\odot .6599$ & $\odot .6737$ \\
\hline & NESTED CLADE & 11.7399 & 0.3336 & 1.0000 \\
\hline CLADE & CN4cn2 (Tip) & & & \\
\hline TYPE & OF DISTANCE & DISTANCE & PROB . $<=$ & PROB. $>=$ \\
\hline & WITHIN CLADE & 0.0000 & 1.0000 & 1.0000 \\
\hline & NESTED CLADE & 35.8318 & 1.0000 & 0.3336 \\
\hline
\end{tabular}

PART III. TEST OF INTERIOR VS. TIP CLADES:

$\begin{array}{rrrr}\text { TYPE OF DISTANCE } & \text { I-T DISTANCE } & \text { PROB. }<= & \text { PROB. }>= \\ \text { WITHIN CLADE } & 11.6594 & 0.6599 & 0.6737 \\ \text { NESTED CLADE } & -24.0919 & 0.3336 & 1.0000\end{array}$

PERMUTATION ANALYSIS OF 1-34 OTAGO SOUTH 1

BASED ON 10000 RESAMPLES

PART I. EXACT CONTINGENCY TEST:
OBSERVED CHI-SQUARE STATISTIC $=11.0000$
THE PROBABILITY OF A RANDOM CHI-SQUARE BEING GREATER THAN OR EQUAL TO THE OBSERVED CHI-SQUARE = 0.1872

PART II. GEOGRAPHIC DISTANCE ANALYSIS:

\begin{tabular}{|c|c|c|c|c|}
\hline \multirow{4}{*}{$\begin{array}{r}\text { CLADE } \\
\text { TYPE }\end{array}$} & \multicolumn{4}{|c|}{ CS1cs1 (Interior) } \\
\hline & OF DISTANCE & DISTANCE & PROB . $<=$ & PROB . > = \\
\hline & WITHIN CLADE & 16.9792 & 0.0960 & 1.0000 \\
\hline & NESTED CLADE & 19.7375 & 0.0960 & 1.0000 \\
\hline \multirow{4}{*}{$\begin{array}{r}\text { CLADE } \\
\text { TYPE }\end{array}$} & cs3cs1 (Tip) & & & \\
\hline & OF DISTANCE & DISTANCE & PROB . $<=$ & PROB. $>=$ \\
\hline & WITHIN CLADE & 0.0000 & 0.6364 & 1.0000 \\
\hline & NESTED CLADE & 46.9392 & 1.0000 & 0.0960 \\
\hline
\end{tabular}

PART III. TEST OF INTERIOR VS. TIP CLADES:

$\begin{array}{rrrr}\text { TYPE OF DISTANCE } & \text { I-T DISTANCE } & \text { PROB. }<= & \text { PROB. }>= \\ \text { WITHIN CLADE } & 16.9792 & 0.0960 & 1.0000 \\ \text { NESTED CLADE } & -27.2017 & 0.0960 & 1.000 \odot\end{array}$

PERMUTATION ANALYSIS OF 1-36 OTAGO SOUTH 2 BASED ON 10000 RESAMPLES

PART I. EXACT CONTINGENCY TEST: 
OBSERVED CHI-SQUARE STATISTIC $=0.7500$

THE PROBABILITY OF A RANDOM CHI-SQUARE BEING GREATER THAN OR EQUAL TO THE OBSERVED CHI-SQUARE $=1.0000$

PART II. GEOGRAPHIC DISTANCE ANALYSIS:

$\begin{array}{rrrr}\text { CLADE CS2CS2 (Interior) } & & & \\ \text { TYPE OF DISTANCE } & \text { DISTANCE } & \text { PROB. }<= & \text { PROB. }>= \\ \text { WITHIN CLADE } & 5.3569 & 1.0000 & 0.6624 \\ \text { NESTED CLADE } & 4.6874 & 1.0000 & 0.6624 \\ & & & \\ \text { CLADE CS4CS2 (Tip) } & & & \\ \text { TYPE OF DISTANCE } & \text { DISTANCE } & \text { PROB.<= } & \text { PROB.>= } \\ \text { WITHIN CLADE } & 0.0000 & 1.0000 & 1.0000 \\ \text { NESTED CLADE } & 2.1871 & 0.6624 & 1.0000\end{array}$

PART III. TEST OF INTERIOR VS. TIP CLADES:

$\begin{array}{lrrr}\text { TYPE OF DISTANCE } & \text { I-T DISTANCE } & \text { PROB. }<= & \text { PROB. }>= \\ \text { WITHIN CLADE } & 5.3569 & 1.0000 & 0.6624 \\ \text { NESTED CLADE } & 2.5003 & 1.0000 & 0.6624\end{array}$

PERMUTATION ANALYSIS OF 2-17 OTAGO 3

BASED ON $1000 \odot$ RESAMPLES

PART I. EXACT CONTINGENCY TEST:

OBSERVED CHI-SQUARE STATISTIC $=34.9143$

THE PROBABILITY OF A RANDOM CHI-SQUARE BEING GREATER THAN OR EQUAL TO THE OBSERVED CHI-SQUARE $=0.1168$

PART II. GEOGRAPHIC DISTANCE ANALYSIS:

\begin{tabular}{|c|c|c|c|c|}
\hline \multirow{4}{*}{$\begin{array}{l}\text { CLADE } \\
\text { TYPE }\end{array}$} & 1-30 (Interior) & & & \\
\hline & OF DISTANCE & DISTANCE & PROB. $<=$ & PROB. $>=$ \\
\hline & WITHIN CLADE & 28.1575 & 0.0000 & 1.0000 \\
\hline & NESTED CLADE & 58.8966 & 0.7136 & $\odot .2864$ \\
\hline \multirow{4}{*}{$\begin{array}{r}\text { CLADE } \\
\text { TYPE }\end{array}$} & 1-33 (Interior) & & & \\
\hline & OF DISTANCE & DISTANCE & PROB. $<=$ & PROB. $>=$ \\
\hline & WITHIN CLADE & $\odot .0 \odot \odot 1$ & $1.000 \odot$ & 0.1513 \\
\hline & NESTED CLADE & 58.9653 & 0.6125 & 0.5388 \\
\hline \multirow{4}{*}{$\begin{array}{r}\text { CLADE } \\
\text { TYPE }\end{array}$} & 1-34 (Interior) & & & \\
\hline & OF DISTANCE & DISTANCE & PROB. $<=$ & PROB. $>=$ \\
\hline & WITHIN CLADE & 24.2711 & 0.0039 & 0.9961 \\
\hline & NESTED CLADE & 55.8308 & 0.3601 & 0.6399 \\
\hline
\end{tabular}

NO INTERIOR/TIP CLADES EXIST IN THIS GROUP 
PERMUTATION ANALYSIS OF 2-18 OTAGO 4

BASED ON 10000 RESAMPLES

PART I. EXACT CONTINGENCY TEST:

OBSERVED CHI-SQUARE STATISTIC $=1.3333$

THE PROBABILITY OF A RANDOM CHI-SQUARE BEING GREATER THAN OR EQUAL TO THE OBSERVED CHI-SQUARE $=1.000 \%$

PART II. GEOGRAPHIC DISTANCE ANALYSIS:

\begin{tabular}{|c|c|c|c|c|}
\hline \multicolumn{5}{|c|}{ CLADE 1-31 (Interior) } \\
\hline \multirow{3}{*}{ TYPE } & OF DISTANCE & DISTANCE & PROB . $<=$ & PROB. $>=$ \\
\hline & WITHIN CLADE & 14.4168 & 0.7510 & 0.7489 \\
\hline & NESTED CLADE & 14.2829 & 0.7510 & 0.7489 \\
\hline \multicolumn{5}{|l|}{ CLADE } \\
\hline \multirow[t]{3}{*}{ TYPE } & OF DISTANCE & DISTANCE & PROB . $<=$ & PROB . $>=$ \\
\hline & WITHIN CLADE & $\odot . \odot \odot \odot \odot$ & $1.0 \odot \odot \odot$ & $1.0 \odot \odot \odot$ \\
\hline & NESTED CLADE & 32.2469 & 1.0000 & $\odot .4999$ \\
\hline
\end{tabular}

PART III. TEST OF INTERIOR VS. TIP CLADES:

$\begin{array}{rrrr}\text { TYPE OF DISTANCE } & \text { I-T DISTANCE } & \text { PROB. }<= & \text { PROB . >= } \\ \text { WITHIN CLADE } & 14.4168 & 0.7510 & 0.7489 \\ \text { NESTED CLADE } & -17.9640 & 0.4999 & 1.0000\end{array}$

PERMUTATION ANALYSIS OF 2-19 OTAGO 5

BASED ON 10000 RESAMPLES

PART I. EXACT CONTINGENCY TEST:
OBSERVED CHI-SQUARE STATISTIC =
$4.000 \odot$
THE PROBABILITY OF A RANDOM CHI-SQUARE BEING GREATER THAN OR EQUAL TO THE OBSERVED CHI-SQUARE $=\quad 0.4078$

PART II. GEOGRAPHIC DISTANCE ANALYSIS:

\begin{tabular}{|c|c|c|c|c|}
\hline \multirow{4}{*}{$\begin{array}{r}\text { CLADE } \\
\text { TYPE }\end{array}$} & \multicolumn{4}{|c|}{ 1-35 (Interior) } \\
\hline & OF DISTANCE & DISTANCE & PROB . $<=$ & PROB. $>=$ \\
\hline & WITHIN CLADE & 6.8033 & 0.3938 & 0.7091 \\
\hline & NESTED CLADE & 17.0512 & 0.7058 & 0.3971 \\
\hline \multicolumn{5}{|l|}{ CLADE } \\
\hline \multirow[t]{3}{*}{ TYPE } & OF DISTANCE & DISTANCE & PROB . $<=$ & PROB . > = \\
\hline & WITHIN CLADE & 3.6456 & 0.1029 & 1.0000 \\
\hline & NESTED CLADE & 14.7999 & 0.2046 & ๑.8983 \\
\hline
\end{tabular}


PART III. TEST OF INTERIOR VS. TIP CLADES:

$\begin{array}{rrrr}\text { TYPE OF DISTANCE } & \text { I-T DISTANCE } & \text { PROB. }<= & \text { PROB. }>= \\ \text { WITHIN CLADE } & 3.1577 & 0.8983 & 0.2046 \\ \text { NESTED CLADE } & 2.2513 & 0.8983 & 0.2046\end{array}$

PERMUTATION ANALYSIS OF 3-10 OTAGO ALL

BASED ON 10000 RESAMPLES

PART I. EXACT CONTINGENCY TEST:

OBSERVED CHI-SQUARE STATISTIC $=\quad 77.5515$

THE PROBABILITY OF A RANDOM CHI-SQUARE BEING GREATER THAN OR EQUAL TO THE OBSERVED CHI-SQUARE $=\quad 0.0 \odot \odot \odot$

PART II. GEOGRAPHIC DISTANCE ANALYSIS:

$\begin{array}{rlrr}\text { CLADE } & \text { 2-17 (Interior) } & & \\ \text { TYPE OF DISTANCE } & \text { DISTANCE } & \text { PROB. }< & \text { PROB. }>= \\ \text { WITHIN CLADE } & 57.5127 & 0.3634 & 0.6366 \\ \text { NESTED CLADE } & 57.5334 & 0.3124 & 0.6876 \\ & & & \\ \text { CLADE 2-18 (Tip) } & & & \\ \text { TYPE OF DISTANCE } & \text { DISTANCE } & \text { PROB.<= } & \text { PROB. }>= \\ \text { WITHIN CLADE } & 16.0793 & 0.0667 & 0.9334 \\ \text { NESTED CLADE } & 35.9937 & 0.0607 & 0.9394 \\ \text { CLADE } & & & \\ \text { TYPE } \text { OF DISTANCE } & & & \\ \text { WITHIN CLADE } & \text { DISTANCE } & \text { PROB.<= } & \text { PROB.>= } \\ \text { NESTED CLADE } & \text { 15.9097 } & 0.0222 & 0.9778 \\ & 86.5554 & 0.9578 & 0.0422\end{array}$

PART III. TEST OF INTERIOR VS. TIP CLADES:

$\begin{array}{rrrr}\text { TYPE OF DISTANCE } & \text { I-T } \text { DISTANCE } & \text { PROB. }<= & \text { PROB. }>= \\ \text { WITHIN CLADE } & 41.5276 & 0.9886 & 0.0114 \\ \text { NESTED CLADE } & -6.5501 & 0.3141 & 0.6859\end{array}$

